Chunlai Wang

Evolution,

Monitoring and

Predicting Models

of Rockburst

Precursor Information for Rock Failure

Springer Open 
Evolution, Monitoring and Predicting Models of Rockburst 
Chunlai Wang

\section{Evolution, Monitoring and Predicting Models of Rockburst}

Precursor Information for Rock Failure

算 Springer Open 


\author{
Chunlai Wang \\ Faculty of Resources and Safety Engineering \\ China University of Mining and Technology \\ Beijing \\ Beijing \\ China
}

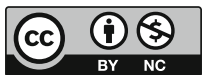

ISBN 978-981-10-7547-6

ISBN 978-981-10-7548-3 (eBook)

https://doi.org/10.1007/978-981-10-7548-3

Library of Congress Control Number: 2017960786

(C) The Editor(s) (if applicable) and The Author(s) 2018. This book is an open access publication.

Open Access This book is licensed under the terms of the Creative Commons Attribution-NonCommercial 4.0 International License (http://creativecommons.org/licenses/by-nc/4.0/), which permits any noncommercial use, sharing, adaptation, distribution and reproduction in any medium or format, as long as you give appropriate credit to the original author(s) and the source, provide a link to the Creative Commons license and indicate if changes were made.

The images or other third party material in this book are included in the book's Creative Commons license, unless indicated otherwise in a credit line to the material. If material is not included in the book's Creative Commons license and your intended use is not permitted by statutory regulation or exceeds the permitted use, you will need to obtain permission directly from the copyright holder.

This work is subject to copyright. All commercial rights are reserved by the author(s), whether the whole or part of the material is concerned, specifically the rights of translation, reprinting, reuse of illustrations, recitation, broadcasting, reproduction on microfilms or in any other physical way, and transmission or information storage and retrieval, electronic adaptation, computer software, or by similar or dissimilar methodology now known or hereafter developed. Regarding these commercial rights a non-exclusive license has been granted to the publisher.

The use of general descriptive names, registered names, trademarks, service marks, etc. in this publication does not imply, even in the absence of a specific statement, that such names are exempt from the relevant protective laws and regulations and therefore free for general use.

The publisher, the authors and the editors are safe to assume that the advice and information in this book are believed to be true and accurate at the date of publication. Neither the publisher nor the authors or the editors give a warranty, express or implied, with respect to the material contained herein or for any errors or omissions that may have been made. The publisher remains neutral with regard to jurisdictional claims in published maps and institutional affiliations.

Printed on acid-free paper

This Springer imprint is published by Springer Nature

The registered company is Springer Nature Singapore Pte Ltd.

The registered company address is: 152 Beach Road, \#21-01/04 Gateway East, Singapore 189721, Singapore 


\section{Preface}

Over the past decades, great progress has been made in scientifically understanding rock failure behavior of rockburst. In particular, advances in field of monitoring and predicting methods of rock failure have led to a profound understanding of rockburst generation processes in terms of underlying physics. However, the world mining and geotechnical engineering scholars have done a lot of researches on the formation mechanism of rockburst, as well as its prediction, prevention, and so on. As the understanding of mechanism and criterion of rockburst just stays at hypothesis and test stage, the theory of rockburst prediction is far from perfect to guide the engineering work. Therefore, it is not appropriate to apply the existing theoretical results directly to solve the problems of rockburst.

This book focuses on investigating predicting precursory information and key points of rockburst through laboratory experiment, theoretical analysis, numerical simulation, and case studies. Understanding the evolution patterns for the microstructure instability of rock is a prerequisite to the rockburst predicting. The book aims at providing a guide for the readers seeking to understand the evolution patterns for the microstructure of rock failure, the predicting key point, and predicting model of rockburst. It will be an essential reference to the most promising innovative rockburst and even other dynamic hazard-predicting technologies.

The layout of monograph is as follows:

Chapter 1: A general introduction followed by an overview of rockburst. Also, the topic of study status on the occurrence conditions of rockburst and predicting means is discussed. What's more, it also gives some further study on predicting rockburst in this chapter.

Chapter 2: A detailed description of laboratory experiments and analytical approaches that have been adopted for the presented study is discussed in this chapter. It primarily contains scanning electron microscope (SEM) and energy spectrum analysis, acoustic emission (AE) experiment, and computed tomography (CT) scanning experiment of rock under loading. All experimental data are the foundation for the further discussion.

Chapter 3: A detailed introduction to rock failure theory and the occurrence mechanism of rockburst is discussed in this chapter. It includes the classical 
rockburst mechanism, the stress-energy formation and occurrence mechanism of rockburst, the nonlinear dynamics theory on rockburst. All of the theories are the foundation to analyze the mechanism and the phenomenon of rockburst.

Chapter 4: Three-dimensional reconstruction model of fissures is presented in rock failure process. It shows the details of the rock cracks extraction and calculation based on the CT images, three-dimensional reconstruction model, and numerical simulation of rock fissures. In this chapter, detailed description of three-dimensional reconstruction model and numerical simulation of rock fissures is given.

Chapter 5: The process of destruction of geotechnical materials is the evolution of microcrack propagation. The complexity, polyphase, and disordered distribution of rock cracks constitute the nonlinearity of rock fracture instability. This chapter established rock system entropy model based on the nonlinear dynamics evolution patterns of crack using CT images, which effectively reveal the mesoscopic mechanisms from crack initiation to propagation. Then, it derives the crack growth factor model through the chaos of crack criterion. This chapter also reveals the pattern of rock damage evolution process of crack propagation.

Chapter 6: This chapter introduced the precursor information of failure of rock fracture, which includes the spatial and temporal evolution, the characteristics of relative quiet period, the energy change characteristics, and the spatial-temporalenergy evolution model of rock failure. Then, it also contains some microseismic events situation in deep mining. All of these information are the foundation of pre-characteristics of rockburst.

Chapter 7: This chapter mainly introduced the experimental investigation of synergetic predicting key points of rockburst using multi-measure monitoring, which includes some theories that had been used in monitoring and predicting rockburst, such as infrared thermography, load/unload energy response ratio theory, entropy theory, tangent damage factor (TDF) identification method, $\mathrm{AE} /$ microseismic activity monitoring theory, and so on. Based on all of these monitoring theories, we obtained the synergetic predicting model of rock failure using multi-measure monitoring methods.

Chapter 8: Bayesian theory, which has been successfully applied in many research fields, provides a clear and a flexible method for making predictions using incomplete knowledge. Fuzzy matter-element theory is primarily used to study the problem of incompatibility. It can be also used for solving multiple parameters evaluation problem by formalizing the problems and establishing the corresponding matter-element. In this chapter, we established rockburst predicting model based on Bayesian theory and fuzzy matter-element theory.

Chapter 9: Some cases were showed to analyze and verify the precursor characteristic of rockburst. It contains the experimental verification and on-site verification. In this chapter, we verified and analyzed the precursor characteristic patterns, the dynamics evolution, the predicting key point, and the predicting model of rockbursts by the microseismic monitoring system in Huize Lead-Zinc mine.

In this monograph, chapters are carefully developed to cover the evolution patterns for the microstructure instability of rock, and we established some 
rockburst hazard monitoring, predicting criterions, and predicting models. The issue with a holistic and systematic approach addressed that predicting investigates of rockburst based on the evolution patterns for the microstructure of rock failure and establishes the predicting model of rockburst. This book is written for researchers of mining engineering, rock mechanics engineering, and safety engineering.

This book was supported by the National Natural Science Foundation of China (Grant Nos. 51374217, 51574246), the National Key Research and Development Project (Grant No. 2017YFC0804201), and the Fundamental Research Funds for the Central Universities (Grant No. 2011QZ01). Finally, I would also like to express my sincere thanks to Prof. Aixiang $\mathrm{Wu}$ (University of Science and Technology Beijing), Chairman Bigen Xu (Changsha Institute of Mining Research Co., Ltd.), Prof. Jianxiong Li and Xiaosheng Chuai, who have greatly improved this book. I am extremely grateful to my family for many years of personal support and understanding. I would like to thank my other students for their outstanding work, including Hui Lu, Zhijiang Lu, Lu Liu, Xiaolin Hou, Ansen Gao, Guangyong Li, Weiqiang Li, Deyang Ba, Bin Liu, Feng Shi, Haojie Xue, Tiancai Bao, Fuli Wang. 
The original version of the book was revised: Incorrect year range in Front matter has been corrected. 


\section{Acknowledgements}

This book was sponsored by National Natural Science Foundation of China (Grant Nos. 51374217, 51574246), the National Key Research and Development Project (Grant No. 2017YFC0804201), and the Fundamental Research Funds for the Central Universities (Grant No. 2011QZ01). 


\section{About the Author}

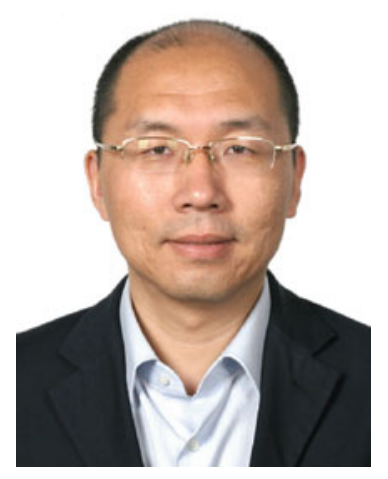

Dr. Chunlai Wang graduated from Heibei Polytechnic University in 2001 and he obtained his $\mathrm{PhD}$ of Mining Engineering from University of Science and Technology Beijing in 2011. He works as associate professor in the Faculty of Resources and Safety Engineering, China University of Mining \& Technology Beijing, China, and he is now the associate director of the department of Department of Resources Engineering. In 2015-2016, he commenced his research in University of British Columbia, as a visiting scholar. In 2012, he was awarded the National Youth Science and Technology Award from China Coal Society. He took charge of more than 10 national scientific projects including the National Natural Science Foundation of China (NSFC). His research interests focus mainly on predicting of mine dynamic hazards, predicting model and criterion, and reinforced technology for 16 years. Up to now, he has published over 50 academic papers and holds 8 patents in China, in which more than 15 English papers were published in International Journal of Rock Mechanics and Mining Sciences, etc. He has also authored a number of books and book chapters including Microseismic Monitoring Predicting and Reinforced Technology of Dynamic Hazards in Deep Mining (Beijing: Metallurgical Industry Press, 2013). He has won 6 National or Provincial Science and Technology Progress Awards. 


\section{Contents}

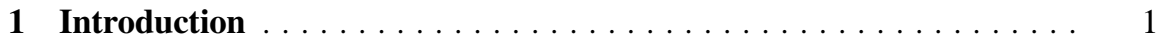

1.1 The Overview of Rockburst $\ldots \ldots \ldots \ldots \ldots \ldots \ldots \ldots \ldots 1$

1.2 Current Status on Rockburst Induced Conditions .......... 2

1.3 Current Status on the Precursor Characteristics of Rockbursts ... 4

1.4 Current Status on the Evolution of Rockburst........... 5

1.5 Current Status on Predicting of Rockburst ............ 6

1.5.1 Study on the Synergetic Monitoring of Rockburst .... 6

1.5.2 Study on the Predicting Model of Rockburst ........ 6

1.5.3 Study on the Field Predicting of Rockburst Hazard ... . 8

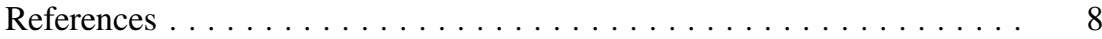

2 Experimental Materials and Equipments $\ldots \ldots \ldots \ldots \ldots \ldots \ldots 11$

$2.1 \quad$ Experimental Materials . . . . . . . . . . . . . . . . . 11

2.2 Experimental Equipments . . . . . . . . . . . . . . . . 13

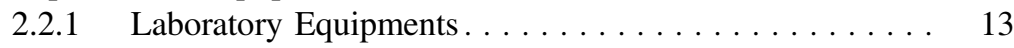

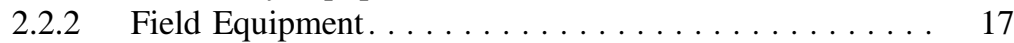

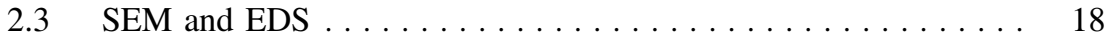

Reference.............................. 22

\section{Mechanism and Predicting Theory-Based of Rockburst}

Evolution ................................ 23

3.1 Introduction ........................ 23

3.2 Mechanism of Rockburst. . . . . . . . . . . . . . . . 24

3.3 Mechanism of Rockburst Stress-Energy Evolution . . . . . . . . 25

3.4 Nonlinear Dynamic Theory of Predicting Rockburst . . . . . . . 27

3.4.1 Mutation Theory ...................... 27

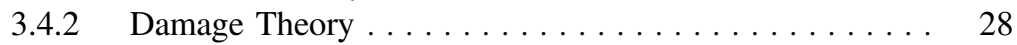

3.4.3 Load/Unload Response Ratio Theory . . . . . . . . . 28

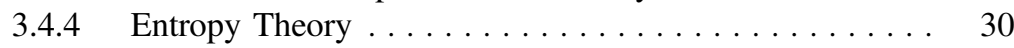

3.4.5 Fuzzy Matter Element Theory . . . . . . . . . . . . 31 
3.4.6 Bayesian Theory and Network Model . . . . . . . . . 32

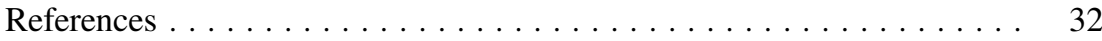

4 Three-Dimensional Reconstruction Model and Numerical

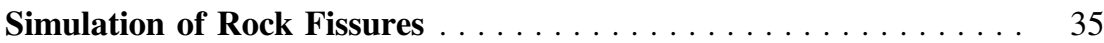

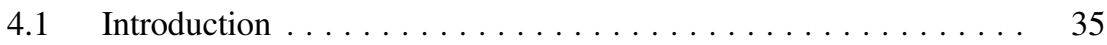

4.2 CT Scanning Experiment Under Uniaxial Cyclic

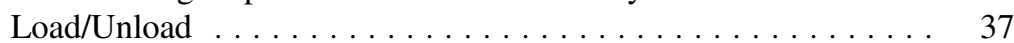

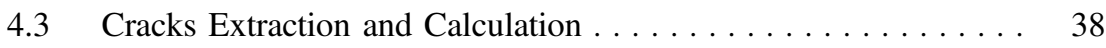

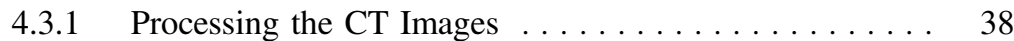

4.3.2 Calculating the Length and Area of Cracks . . . . . . . . 42

4.4 Three-Dimensional Reconstruction Model and Numerical

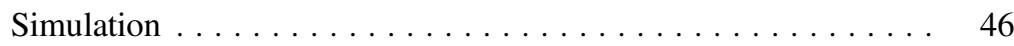

4.4.1 Establishing a Three-Dimensional Finite Element Model . . . . . . . . . . . . . . . . . . . . 47

4.4.2 Numerical Simulation of Fissured Rock. . . . . . . . . 50

References . . . . . . . . . . . . . . . . . . . . . . . . . 57

5 Experimental Investigation on Nonlinear Dynamic Evolution

Patterns of Cracks in Rock Failure Process . . . . . . . . . . . . . . 59

$5.1 \quad$ Introduction . . . . . . . . . . . . . . . . . . . . . . 59

5.2 Pattern of Nonlinear Dynamics Evolution of Rock Cracks . . . . 60

$5.2 .1 \quad$ Fractal Theory . . . . . . . . . . . . . . 61

5.2.2 Results Analysis on Fractal Dimension of Rock Cracks .................... 61

5.2.3 Entropy Model of Rock System Based on Fractal Dimension ...................... 65

5.3 Chaotic Characteristics of Cracks Evolution in Rock Failure

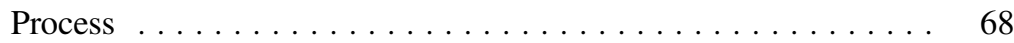

5.3.1 Chaos Theory .................. 68

5.3.2 Discrimination of Chaotic Characteristics Defined by Li-Yorke. . . . . . . . . . . . . . . . . . 69

5.3.3 Construction of Crack Growth Factor Model . . . . . . . 72

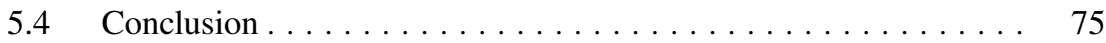

References . . . . . . . . . . . . . . . . . . . . . . . . . 75

6 Experimental Investigation on AE Precursor Information

of Rockburst . . . . . . . . . . . . . . . . . . . . . . . . . . . . . . 77

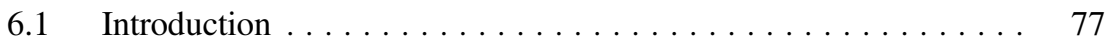

6.2 Spatio-temporal Evolution Pattern of Rock Failure . . . . . . . . 78

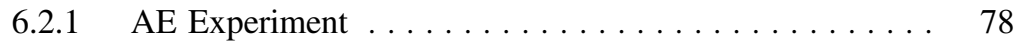

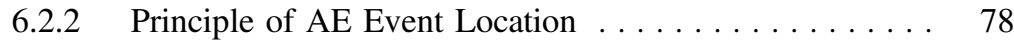

6.2.3 Spatio-temporal Distribution Characteristics of Rock

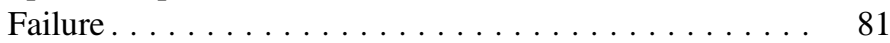

6.3 Characteristics of Relatively Quiet Period for Rock Failure . . . . 82 
6.3.1 Deformation and Failure Characteristics of Loaded

Rock ....................... 82

6.3.2 Relationship Between the Constitutive Characteristics and the Accumulative AE Counts ............. 84

6.3.3 Relationship Between AE Rate and Constitutive Characteristics .................... 85

6.3.4 Evolution Characteristics of AE Amplitude in the Time Domain . . . . . . . . . . . . . . . . . 86

6.3.5 Analyzation of Precursory Characteristics of Rock

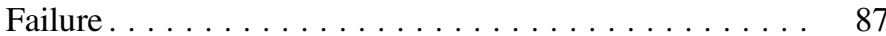

6.4 Variation Regulation of AE Energy Parameters for Rock

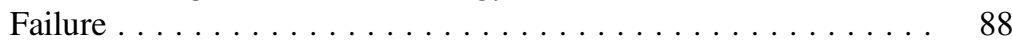

6.4.1 Experimental Setup and Procedure . . . . . . . . 88

6.4.2 Energy Parameters Variations in Each Channel . . . . . . 88

6.4.3 Relationship of Energy and Stress-Strain Curve on Time Domain. . . . . . . . . . . . . . . . . . . 89

6.4.4 Analysis of Damage Features . . . . . . . . . . . . . . 90

6.4.5 Energy Release Rate of Rock Damage . . . . . . . . . . . 92

6.5 Evolution Patterns of Spatial-Temporal-Energy on Rock

Fracture Surface . . . . . . . . . . . . . . . . . . . . . 93

6.5.1 Experimental Setup and Procedure . . . . . . . . . . 94

6.5.2 Relationship Between Spatial Distribution of AE Events and Rock Fracture . . . . . . . . . . . . . . 94

6.5.3 Spatial-Temporal Evolution of Strong AE Events on Rock Fracture . . . . . . . . . . . . . . . . 96

6.5.4 Spatial-Temporal-Energy Evolution Model of Strong AE Events in Limestone Fracture . . . . . . . . . . . . 98

6.5.5 Spatial Fractal Dimension Evolution Model of AE Events on Fracture ................. 101

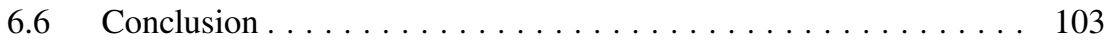

References . . . . . . . . . . . . . . . . . . . . . . . . . . . . . . . . . . 104

\section{Experimental Investigations on Multi-means and Synergistic}

Prediction for Rockburst . . . . . . . . . . . . . . . . . . . . . . . 107

$7.1 \quad$ Introduction . . . . . . . . . . . . . . . . . . . . . . . 107

7.2 Predicting Points of Infrared Precursor for Coal Failure . . . . . . . 109

7.2.1 Laboratory Test of Coal Failure . . . . . . . . . . . . . . . . 109

7.2.2 Theoretical Bases of IRR Detection. . . . . . . . . . . 110

7.2.3 IRT Characteristics of Coal Samples . . . . . . . . . . . 112

7.2.4 IRT Prediction for Rock Failure . . . . . . . . . . . . 117

7.3 Experimental Investigation on Predicting Rock Failure Using Load/Unload Energy Response Ratio Theory . . . . . . . . . . . . . 118

7.3.1 LURR Characteristics of Coal Samples . . . . . . . . . 118

7.3.2 LURR Prediction of Coal Failure . . . . . . . . . . . 121 
7.4 Experimental Investigation on Predicting Points Using Tangent

Damage Factor for Rock Failure . . . . . . . . . . . . . . . . . 122

7.4.1 Laboratory Test of Rock Failure . . . . . . . . . . . . . . 122

7.4 .2 Define the TDF . . . . . . . . . . . . . . . . . . . 123

7.4.3 Damage Characteristic of Limestone Failure . . . . . . . 126

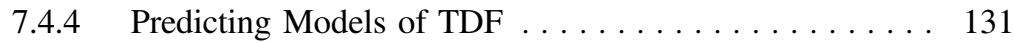

7.5 Experimental Investigation on Predicting Points Using

Information Entropy Theory for Rock Failure . . . . . . . . . . 133

7.5.1 AE Dominant Frequency . . . . . . . . . . . . 133

7.5.2 AE Dominant Frequency Entropy . . . . . . . . . . . . . . 134

7.5.3 AE Dominant Frequency and Entropy . . . . . . . . . 135

7.6 Predicting Key Point Identification Using Traditional

Monitoring Method........................ 137

7.6.1 Methods for Instrument Installation and Data

Collection . . . . . . . . . . . . . . . . . 137

7.6.2 Recognition Method of Predicting Hazards . . . . . . . . 138

$7.7 \quad b$ Value Characteristics with MS Activity in Deep Mining . . . . . 139

7.7.1 The Magnitude-Frequency Relation $-b$ Value . . . . . . . 139

7.7.2 Changes of $b$ Value Caused by Rockmass

Excavation. . . . . . . . . . . . . . . . . . . 140

7.8 Identification of Predicting Key Point Using

AE/MS Monitoring System . . . . . . . . . . . . . . . . . 141

7.9 Model of Multi-means and Synergistic Prediction

for Rockburst . . . . . . . . . . . . . . . . . . . . . . . . . . . . . . 143

7.10 Conclusion . . . . . . . . . . . . . . . . . . . . . . . . 144

References . . . . . . . . . . . . . . . . . . . 145

8 Predicting Model of Rockburst Based on Nondeterministic

Theory . . . . . . . . . . . . . . . . . . . . . . . . . . . 149

$8.1 \quad$ Introduction . . . . . . . . . . . . . . . . . . . 149

8.2 Predicting Model of Rockburst Based on Bayesian Theory . . . . . 151

8.2.1 An Overview of Bayesian Theory . . . . . . . . . . . 151

8.2.2 Key Factors of Rockburst Tendency . . . . . . . . . . . 153

8.3 Predicting Model of Rockburst Based on Fuzzy Matter-Element

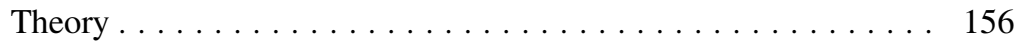

8.3.1 Fuzzy Matter-Element and Composite

Fuzzy Matter-Element . . . . . . . . . . . . 156

8.3.2 Standard Fuzzy Matter-Element and Difference Square Composite Fuzzy Matter-Element . . . . . . . . . . . 157

8.3.3 Weight Coefficients Determined by Entropy Method . . . 157

8.3.4 Closeness Degree and Comprehensive Evaluation ..... 158

8.4 Conclusions . . . . . . . . . . . . . . . . . . . . . . . . . . 159

References . . . . . . . . . . . . . . . . . . . . . . . . . . . . 159 


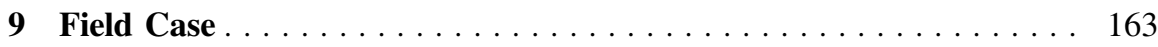

9.1 Design of Microseismic Monitoring System in Huize Lead-Zine

Mine . . . . . . . . . . . . . . . . . . . . . . . . 163

9.1.1 Main Influence Factor . . . . . . . . . . . . . . . . . . . 164

9.1.2 Microseismic Monitoring System . . . . . . . . . . . . 164

9.1.3 Sensor Arrangement Design . . . . . . . . . . . . . 165

9.2 Case Verification . . . . . . . . . . . . . . . . . . . 165

9.2.1 Predicting Case Based on Displacement Nephogram ... 165

9.2.2 Predicting Case Based on Apparent Stress . . . . . . . . . 167

9.3 Predicting Key Points Identification of Dynamic Hazard . . . . . . 168

9.3.1 Dynamic Hazard Predicting Using Routine

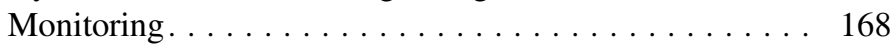

9.3.2 Predicting Key Point Identification Using

CAV and MS Events Activity. . . . . . . . . . . . . . 169

9.4 Predicting Model of Rockburst Based on Bayesian Theory ... . . 172

9.4.1 Training Samples . . . . . . . . . . . . . . . . 172

9.4.2 Predicting Rockburst Tendency Using a Bayesian

Model ... . . . . . . . . . . . . . . . . . . 172

9.4.3 Verifying Accuracy of Bayesian Model ........... 175

9.5 Predicting Model of Rockburst Based on Fuzzy Matter

Element Theory . . . . . . . . . . . . . . . . . . . . . 177

9.5.1 Testing and Field Condition . . . . . . . . . . . . 177

9.5.2 Predicting Rockburst Tendency . . . . . . . . . . . . 181

9.5.3 Predicting Model of Fuzzy Matter-Element Theory ... . 184

9.6 Conclusion . . . . . . . . . . . . . . . . . . . . . . . 187

References ........................... 188 


\section{Chapter 1 \\ Introduction}

\subsection{The Overview of Rockburst}

Mine dynamic hazards mainly include the rockburst, high-speed landslide, coal and gas outburst, underground debris flow, gob area catastrophic collapse, tailings dam collapse, and water inrush, etc. Rockburst is usually referred to as dynamic catastrophe, which results in causalities and roadway destruction, caused by elastic strain energy emitting in a sudden, rapid and violent way from coal or rockmass. It is often accompanied by an airblast and violent failures which can disrupt mine ventilation, pose a danger to miners, and may also cause a large release of strata gas and explosive dust into the air (Brauner 1994; Dou et al. 2012; Jiang et al. 2014; Cook 1976; Salamon 1983; Ortlepp 1994).

With the depletion of shallow mineral resources, there is an inevitable trend for mineral extraction to go deeper. Duo to the mining depth and the mined out area increases, the frequency and intensity of induced explosion will be increased simultaneously. Mining face and deep tunnels near the rockburst area will endanger the production safety. Strong rockbursts also cause engineering loss, damage people's living environment and pose a threat to public safety. Therefore, it is of great significance to carry out the research and management of rockburst in deep mining and tunnel engineering (He et al. 2005; Zhou et al. 2005).

Rockburst is one of the most important mine dynamic hazards in China. According to the State Administration of Work Safety of China, the total casualty in mine accidents was 54,744 from 2002 to 2016, especially the accidents caused by the rockburst, water inrush, coal gas outburst and other dynamic hazards account for more than $60 \%$. In 2016, there were 538 deaths in coal mines in China, compared with 9 in the United States. The death totally in China is 59.78 times than that 
of the United States, and about $80-90 \%$ of world's major mining hazards (more than 10 people) occur in China every year.

The study of type and induced condition of rockburst is the precondition for the prevention of rockburst. It was stated that any rockburst results from a mining tremor but not all the tremors cause rockburst. In fact, two kinds of hazards (seismic hazard and rockburst hazard) were proposed and defined, which one tremor cannot be predicted (Kornowski et al. 2012). The tremors in corresponding to the rupture events were observed at scales ranging from laboratory samples to the earth's crust, which included rock failures in laboratory tests and field experiments, landslides, mining-induced seismicity, and crustal earthquakes (Amitrano 2012). It was verified that rocks under stress emit acoustic waves in the laboratory and microseismic events in mines. Then, AEs of rocks observed in the laboratory were considered as a small-scale model of seismicity of earth's crust (Filimonov et al. 2005; Hardy 2003; Scholz 1968).

In the past few years, although the mining industry has carried out a large number of mine rockburst induced conditions and control technology research, the mine safety production situation is still grim. If the mine rockburst induced conditions, monitoring and predicting model can not be solved in short term, it will become a bottleneck restricting for the development of mining (especially deep mining).

\subsection{Current Status on Rockburst Induced Conditions}

In order to solve the key technical problems related to rockbursts, it is necessary to analyze their inducing conditions. Studies have already been done to determine these inducing conditions and to lay foundations for rockburst prediction. However, these studies are incomplete. To date, there is still no unified explanation for the rock dynamic hazards, especially the triggered conditions of rockbursts. So, it is very important to study the rockburst induced conditions and analyze the rock failure behavior by various theories, which can provide reasonable suggestions for mining design, mining method and rockburst prevention measures. It also is very important meaning to mining safety and efficient recovery.

Rockburst is a nonlinear dynamic process, in which the energy is converted into kinetic energy by the broken rockmass and ejected. Thus, from the energy point of view, rockburst is an essentially process, in which the elastic strain energy accumulated in the rockmass is released abruptly. Hence, the main factors that affect the occurrence of a rockburst are the impact which can make rock into a high-energy storage. High-energy storage of rock is more likely to cause rockburst. In other words, rock has sufficient capacity to store large elastic strain energy and there is a high concentration of stress within the rock. Based on this, rockburst was 
induced by both a high concentration of stress and a large quantity of recoverable strain energy (Qian 2004).

At present, relatively few studies focus on the conditions of rockburst and each of them has different emphases. In this area of study work is as follows:

It was established an analytical model for the nonlinear instability mechanism of coal and rockmass which based on non-equilibrium thermos dynamics and dissipative structure theory, the three-dimensional model of coal and rockmass instability, coal and rock damage model by considering the dynamic instability model according to the Hoek-Brown strength theory (Jiang et al. 2005). It was found that the migration of microseismic events was mainly caused by stress redistribution, which was related to the collapse of the main caves, while the secondary microseismic clusters were related to the small-scale damage (Trifu et al. 2008). It was found that main non-uniformity stress would appear after injecting large-volume fluid into the granite body and along the fault surface shear stress would be released. The occurrence of microseismic events and fault surface shape indicating the fault sliding was quasi-static (Scotti et al. 1994). It was also found that a microseismic event with a magnitude of less than 0 can be used to assess a 2.9 level destructive rockburst event. The stress inversion and the main quantification component analysis were of good correlation. What's more, it could be used to derive microseismic failure surfaces and structural maps (Ttifu et al. 1996). It was found that $\mathrm{AE}$ can be used to monitor the material destruction process and quantify the waveform analysis of microseismic event magnitude (Arastehl et al. 1997). AE event was occured in the direction of rupture tip during loading. At the same time, the macro-rupture was occurred at the unloading event. A typical loading event was an essentially component in the tensile stress zone, while the unloading usually was a shear stress.

From a research perspective, the deep mining rockburst were mainly concentrated on the induced conditions. The research work so far mainly analyzed the lab experiment under the uniaxial condition and deduced the model which mainly included the time-consuming, energy storage and energy consumption, the distribution of precursor information before rock failure. However, multiple factors of rockmass, which leaded to rockburst in the deep mining, were in complex stress environment conditions. Actually, lab experiment is only a special case of scene and it has a certain difference compared the actual rockburst, experimental investigation is a similar understanding for the real situation of rockburst hazard. At present, rock mechanics is still not enough explaining to the induced factors of rockburst, the monitoring criterion and the predicting model. Especially, the excavation leads to the stress redistribution and the fault activity induced by the microseismic event is still insufficient. It is necessary to do some further study in the lab experiments and practical production applications. Therefore, it is very important to analyze the characteristics, influencing factors, formation mechanism; emissive events induced condition, rockburst key predicting point and predicting model. 


\subsection{Current Status on the Precursor Characteristics of Rockbursts}

The macroscopic fracture and failure of rockmass are due to the nucleation, expansion and penetration of microfracture. The secondary stress redistribution of rockmass by excavation is affected by the stress field disturbance and resulting in dislocation, disintegration and migration of mesome. With the development of rock mechanics, it has become an important method of dynamic hazards monitoring that includes the monitoring of stress wave and the maturation of $\mathrm{AE}$ positioning technology, microseismic monitoring technology and AE technology. By analyzing the strength, the frequency and the location of microseismic activity, we can get access to some hazards precursor information to achieve risk assessment and predicting. However, the implementation of rock test on the site is more difficult and can't easily get the results. At the same time, lab experiment is an effective way to study the destruction and regularity of rockmass. So, it is an effective means to conduct AE test and combine with the field monitoring results. Therefore, it is the primary task to carry out the research on the prediction of dynamic hazards by summing up the characteristics and evolution patterns of $\mathrm{AE}$ in the process of rock rupture.

To date, less methods aim at studying the distribution of precursory information of rockburst and they have different emphases. The main work in this area as follows:

In a uniaxial loading test, it is known that the number of AE events is small during the initial loading stage. In the later stage of rock elastic and plastic deformation, the number of cumulative AE events increased rapidly before the damage and maintain a low release rate during the rock fracture process (Liu et al. 2011). From the experimental data, it was found that the precursory characteristics of rock samples were inferred and the heterogeneous fault was caused by the failure of specimens (Lei et al. 2004). It was found that the fault, stress and boundary deformation had a close interaction for the shear strain generation area evolved (Mekinnon 2006). It was also found the presence of AE relatively quiet period by rock uniaxial compression AE test (Schiavi et al. 2011). It was found that the AE energy of material was accelerated after destruction through the AE monitoring tests on concrete, rock and other materials under uniaxial loading conditions (Carpinteri et al. 2008).

This book focus on the rockburst induced conditions and predicting, three-dimensional reconstruction model of rock fracture cracks under loading, dynamic evolution of rock fractures, experimental investigation of rockburst precursor information. The author's work in investigation of key points of synergetic predicting of rockbursts and their predicting models based on the nondeterministic theory is also introduced. 


\subsection{Current Status on the Evolution of Rockburst}

Rockburst is a sudden, unexpected and abrupt failure. It is a bottle neck for any geotechnical engineering being carried out at great depth. The study of rock failure process and evolution patterns has become the important issues in underground engineering. In the past, it was difficult to observe the true geometric distribution within the materials due to the limitations of test technique and equipment. With the rapid development of science and technology, Computed Tomography (CT) scanning technology has gradually become the most advanced non-destructive testing means. The application of CT scanning technology can achieve the continuous scan, dynamic observation test under load in the geotechnical engineering. In the same time, we also can get the parameters change in the process of computed tomography, the internal structure of sample, the microscopic structure of sample, the moving microscopic particulate matter, the developmental crack, the local change density, etc. CT image is a kind of grayscale image which can reflect the change of density inside the rockmass. Through the change of gray degree, the distribution of material in different areas of rockmass can be reflected in the image. What's more, the process of structural failure can be well reflected by CT images.

In fact, many researchers analyzed CT images and three-dimensional cracks reconstruction (Kawakata et al. 1999, 2000), and it was come true that to simulate the outdoor rockmass distribution by X-ray rock CT scanning (Ueta et al. 2000). Chinese scholars focus on the analysis of changes of CT number in the CT image and the quantitative analysis was only in theory. The advantages of digital image processing technology did not totally display in the field of geotechnical engineering. It is necessary to use numerical simulation to describe the geotechnical material and reflect the real force of rockmass. Therefore, the CT image of rock, based on the digital image technology, provides a new way to analyze the process of meso-cracks reconstruction.

The process of rock failure is a significant accumulation of process, which is determined by the brittle characteristics of rock. In the process of cyclic load/ unload, the higher concentrated stress will appear at the tip of rock crack, which will lead to the initiation and expansion of cracks inside the rock and gradually generate new macroscopic cracks leading to the overall destruction of specimen. With the continuous development of society and the process of human production, engineering construction is gradually increased in the influence of fractured rockmass.

Most scholars studied the cracks evolution by similar model test which could be observed intuitively and clearly about the process of cracks initiation, expansion and penetration failure. It was conducted the prefabricated oblique fissures of marble compression test which showed the process of expansion and it was asymmetric (Nolen-Hoeksema et al. 1987). What's more, rock failure and the surface of crack distribution were observed in the test. As the existing technical level, internal cracks in the rock sample were achieved, but rock similar materials were used to study the crack propagation and transparent characteristics. All of 
these results in accurate observation and record was not achieved. In this book, CT scanning technology and digital image technology were used to obtain these cracks images of rock under different load levels. The nonlinear dynamics of cracks propagation process was revealed.

The study of propagation evolution patterns still rests in the whole process of crack initiation-extension-failure of microscopic level so far, and it does not explain the macroscopic failure criterion of rock in the process of crack propagation. This book intends to establish a three-dimensional visualization model of cracks which can provide a new way to study the transition from microscopic scale to macroscopic scale.

\subsection{Current Status on Predicting of Rockburst}

\subsubsection{Study on the Synergetic Monitoring of Rockburst}

In underground excavation process, rockburst caused a serious of threat to the construction workers and equipments. The predicting of rock damage and failure has become a problem to be solved urgently for the safe and efficient production of mine which has aroused the attention of many scholars. In fact, the predicting of rock failure has become an important topic in underground engineering field.

As the implementation of on-site rock load/unload test is difficult, laboratory test methods are often used in its replacement. Lab tests and engineering applications used in combination can fulfill deep level study in rock deformation behavior and predicting research. Therefore, it is of great significance to carry out the research on rockburst failure predicting in the laboratory. In addition, the existing rock failure predicting technology means a single application. Hence, it is very important to establish a multi-means to monitor the rockburst. In this book, we do some studies on dynamic hazard evolution, predicting model using traditional monitoring, AE monitoring, microseismic monitoring and infrared monitoring.

\subsubsection{Study on the Predicting Model of Rockburst}

Rockburst predicting is the basis of prevention and control of rockburst hazard. According to the predicting results, the feedback design of rock engineering and the control measures can be taken in time. It is of great theoretical and practical value for the safe and efficient recovery of deeply buried resources. Rockburst tendency evaluation is the prerequisite and basis of predicting hazard.

Bayesian theory, which was successfully applied in many research fields, provided a clear and a flexible method for making predictions using incomplete knowledge. Heckerman (1990) used a Bayesian framework to improve the process 
of medical diagnosis. Making full use of its strong information processing ability, a Bayesian network was applied to the monitoring and management of industrial production processes. A Bayesian model was utilized for choosing investment ventures, and displayed a good ability to cope with future uncertainty. In addition, Bayesian theory was used to identify faults in a computer system. Bayesian theory was demonstrated to be a reliable approach to address complex problems involving many variables with large uncertainties, and models that considered a multi-parameter space were better suited to predicting rockburst tendency than single-variable models. In these studies, the main factors affecting of risk and intensity of rockbursts were used to make a Bayesian model (Heckerman 1990; Weidl et al. 2003; Kemmerer et al. 2002; Jensen et al. 2001).

Matter-element analysis theory is primarily used to study the problem of incompatibility. It was used to solving multiple parameter evaluation problem by formalizing the problem and establishing the corresponding matter-element (Cai 1994; Chen et al. 2007; David and Wen 1997). The improved fuzzy matter-element evaluation method was used to assess water quality, which achieved more reliable results than that using the traditional method. Based on the matter-element method, He et al. (2011) designed a model to evaluate the urban power network planning, and Zhu et al. (2010) analyzed coefficients of evaluation in rockburst. The empirical analysis showed that this model was reliable and feasible. In this book, the main influencing factors of rockburst are considered based on the concept of matter-element analysis in combination with the fuzzy set and closeness degree patterns. The entropy method is also integrated in the weight calculation of this model. An integrated rockburst multi-index evaluation model is established and used to predict the rockburst tendency.

At present, there are many methods to judge rockburst tendency, such as stress method, rockmass integrity coefficient method, strength criterion discrimination method, rock fragility index method, elastic energy index method, dynamic failure time method, rockburst energy ratio method, impact energy index method, impact tendency criterion method, resistivity method and so on. All of these methods and judgment indexes are only considered the individual factors which will produce the one-sidedness and limitations of predicting results. In fact, all aspects will make the problem complicated. So, it is necessary to use the nonlinear mathematical method to comprehensively consider the interrelated factors of rockburst and analyze the rockburst tendency. However, the Bayesian predicting model and the fuzzy predicting model with high accuracy are established by using the Bayesian method and the fuzzy matter-element theory which considering more important influencing factors of rockburst. The Bayesian predicting model considers the rock strength index $R_{b}$, the surrounding rock stress index $R_{\theta}$ and the energy index $W_{\text {et }}$, etc. The fuzzy element predicting model considers four rockburst influencing factors which contains the brittleness coefficient $B$, the impact tendency energy $W_{C F}$, the rock integrity coefficient $K_{V}$ and the rock strength stress ratio $R_{c} / \sigma_{1}$, etc. In this model, the 
interrelated factors of rockburst are considered comprehensively and the predicting results is coincidence with the field.

\subsubsection{Study on the Field Predicting of Rockburst Hazard}

Due to the complexity of rock mechanics behavior in deep mining, the actual situation of deep rock mechanics behavior is difficult to be characterized by theoretical calculation and analysis before the mining implementing. At the same time, the theoretical calculation itself can not reflect the dynamic changes of rock engineering conditions in real time. Therefore, it is a reliable and effective technical measure to master and evaluate the active status of rockmass excavation engineering in deep mining.

In order to predict the dynamic hazard in mining, microseismic monitoring technology has become the main technical means to realize the predicting dynamic hazard in deep mining. Using the microseismic monitoring system, the samples can be used to detect the elastic wave emitted by the rock ruptures. The waveform can determine the coordinates, the intensity and the frequency of microseismic activity. Based on microseismic monitoring technology, it can be used to determine the potential micro-earthquake activity criterion and to achieve predicting by the micro-rupture information. Microseismic monitoring is widely used to provide strong safety protection in deep mining.

\section{References}

Amitrano D (2012) Variability in the power-law distributions of rupture events. Eur Phys J Spec Top 205:199-215

Arasteh MM, Chudnovsky A, Dudley JW (1997) Broadband acoustic emission observations during fracture propagation in rock-like material. Int J Rock Mech Min Sci 34(3/4):1-13

Brauner G (1994) Rockbursts in coal mines and their prevention. AA Balkema, Rotterdam

Cai W (1994) Matter element model and its application. Scientific and Technical Documents Publishing House, Beijing (in Chinese)

Caipinteri A, Lacidogna G, Puzzi S (2008) Prediction of cracking evolution in full scale structures by the $b$ value analysis and Yule statistics. Phys Mesomech 11(5):260-271

Chen HQ, Wang N, Sheng YR (2007) Application of fuzzy matter element model in evaluation of water security based on Euclid approach degree. J Xi'an Univ Technol 23:37-42 (in Chinese)

Cook NG (1976) Seismicity induced by mining. Eng Geol 10:99-122

David KW Ng, Wen Cai (1997) Treating non-compatibility problem from matter element analysis to extenics. ACM SIGICE Bulletin 22(3):2-9

Dou L, Chen T, Gong S, He H, Zhang S (2012) Rockburst hazard determination by using computed tomography technology in deep workface. Saf Sci 50:736-740

Filimonov Y, Lavrov A, Shkuratnik V (2005) Effect of confining stress on acoustic emission in ductile rock. Strain 41:33-35

Hardy HR (2003) Acoustic emission/microseismic activity. AA Balkema Publishers, Rotterdam

He MC, Xie HP, Peng SP et al (2005) Study on rock mechanics in deep mining engineering. Chin J Rock Mech Eng 24(16):2803-2813 
He YX et al (2011) Risk assessment of urban network planning in china based on the matterelement model and extension analysis. Int J Electr Power Energy Syst 33:775-782

Heckerman D (1990) Probabilistic similarity networks. Networks 20(5):607-636

Jensen FV, Kjærulff U, Kristiansen B et al (2001) The SACSO methodology for troubleshooting complex systems. AI EDAM 15(04):321-333

Jiang YD, Zhao YX, Liu WG et al (2005) Investigation on three-dimensional model of instability of translatory coal bumps in deep mining. Chin J Rock Mech Eng 24(16):2864-2869

Jiang Y, Pan Y, Jiang F, Dou L, Ju Y (2014) State of the art review on mechanism and prevention of coal bumps in China. J China Coal Soc 39:205-213

Kawakata H, Cho A, Kiyama T et al (1999) Three-dimensional observations of faulting process in Westerly granite under uniaxial and triaxial conditions by X-ray CT scan. Tectonophysics 313 (3):293-305

Kawakata H, Cho A, Yanagidani T et al (2000) Gross structure of a fault during its formation process in Westerly granite. Tectonophysics 323(1):61-76

Kemmerer B, Mishra S, Shenoy PP (2002) Bayesian casual maps as decision aids in venture capital decision making: methods and applications. Acad Manage 1:C1-C6

Kornowski J, Kurzeja J (2012) Prediction of rockburst probability given seismic energy and factors defined by the expert method of hazard evaluation (MRG). Acta Geophys 60:472-486

Lei X, Koji M, Osamu N et al (2004) Detailed analysis of acoustic emission activity during catastrophic fracture of faults in rock. J Struct Geol 26:247-258

Liu JP, Li YH, Yang YJ (2011) Rock damage process based on acoustic emission monitoring under cyclic loading. J Northeast Univ Nat Sci 32(10):1476-1479

Mckinnon SD (2006) Triggering of seismicity remote from active mining excavations. Rock Mech Rock Eng 39(3):255-279

Nolen RC, Gordon RB (1987) Optical detection of crack patterns in the opening-mode fracture of marble. Int J Rock Mech Min Sci 24(2):135-144

Ortlepp WD, Stacey TR (1994) Rockburst mechanisms in tunnels and shafts. Tunn Undergr Space Technol 9(1):59-65

Qian QH (2004) The current development of nonlinear rock mechanics the mechanics problems of deep rock mass. In: Chinese Society for Rock Mechanics and Engineering (ed) Proceedings of the 8th conference on rock mechanics and engineering, Beijing Science Press

Salamon MDG (1983) Rockbutst hazard and the fight for its alleviation in South Africa gold mines. Institution of Mining and Metallurgy, London

Scholz C (1968) The frequency-magnitude relation of micro fracturing in rock and its relation to earthquakes. Bull Seismol Soc Am 58:399-415

Schiavi A, Niccolini G, Tarizzo P et al (2011) Waveforms and frequency spectra of elastic emissions due to macro fractures in solids. Exp Appl Mech (Springer New York)

Scotti O, Cornet FH (1994) In Situ, Evidence for fluid-induced aseismic slip events along fault zones. Int J Rock Mech Min Sci Geomech Abstr 31(4):347-358

Trifu CI, Urbancic TI (1996) Fracture coalescence as a mechanism for earthquakes observations based on mining induced micro seismicity. Tectonophysics 261(1-3):193-207

Trifu CI, Shumila V (2008) Microseismic monitoring of a controlled collapse at Ocnele Mari, Romania. The 42nd U. S. Rock Mechanics-2nd U.S.-Canada Rock

Ueta K, Tani K, Kato T (2000) Computerized X-ray tomography analysis of three-dimensional fault geometries in basement-induced wrench faulting. Eng Geol 56(1):197-210

Weidl G, Madsen AL, Dahlquist E (2003) Object oriented Bayesian networks for industrial process operation. First Bayesian Applications Modeling Workshop, http://www.intel.com/ research/events/UAI03_workshop 
Zhou HW, Xie HP, Zuo JP (2005) Development in researches on mechanical behaviors of rock under the condition of high ground pressure in the depths. Adv Mech 35(1):91-99

Zhu JR et al (2010) Application of fuzzy matter-element model based on coefficients of evaluation in rock burst. J Hunan Univ Sci Technol

Open Access This chapter is licensed under the terms of the Creative Commons Attribution-NonCommercial 4.0 International License (http://creativecommons.org/licenses/by-nc/ 4.0/), which permits any noncommercial use, sharing, adaptation, distribution and reproduction in any medium or format, as long as you give appropriate credit to the original author(s) and the source, provide a link to the Creative Commons license and indicate if changes were made.

The images or other third party material in this book are included in the book's Creative Commons license, unless indicated otherwise in a credit line to the material. If material is not included in the book's Creative Commons license and your intended use is not permitted by statutory regulation or exceeds the permitted use, you will need to obtain permission directly from the copyright holder.

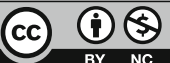




\section{Chapter 2 \\ Experimental Materials and Equipments}

\subsection{Experimental Materials}

Specimens, got from Da'anshan coal mine shaft groove of 9th return air roadway roof and floor strata, were used in the experiment. The top and bottom of roadway were mainly sandstone; the coal seam were of semi dark type, granular structure, medium hardness and good coal quality. Coal specimens were cylindrical cores with a diameter of $50 \mathrm{~mm}$ and a length of $100 \mathrm{~mm}$. The two end planes of every specimen were parallel within an accuracy of $\pm 0.05 \mathrm{~mm}$, and both planes were perpendicular to the longitudinal axis with an accuracy of $\pm 0.25^{\circ}$ (according to ISRM testing guidelines).

The Da'anshan coal mine, a typical in situ stressed mine, is located $50 \mathrm{~km}$ west of Beijing, China, as shown in Fig. 2.1a. The length of Da'anshan mine field is $9 \mathrm{~km}$, the width is $2-4 \mathrm{~km}$, and the area is $25.5 \mathrm{~km}^{2}$. The main geological structure of mine field is the SW-NE synclinal fold. There are many strike and incline faults, contact fracture zones and secondary tectonic features. Some coal seams are separated by a few rock layers, as shown in Fig. 2.1b. The first coal seam, marked as $\# 13$, has a thickness varying from 0.77 to $2.53 \mathrm{~m}$. Below the \#13 coal seam, the material is comprised of a sandy conglomerate with a thickness varying between 0.1 and $0.2 \mathrm{~m}$. Below the sandy conglomerate lies the \#12 coal seam, with a thickness varying from 0.64 to $2.41 \mathrm{~m}$. Below the \#12 coal seam lies a layer of siltstone and sandstone with a thickness of $0.65-1.14 \mathrm{~m}$. The \#10 coal seam is below the siltstone and sandstone, with a thickness ranging from 0 to $7.41 \mathrm{~m}$. Below the \#10 coal seam, there is a layer of siltstone and sandstone with a thickness of 1.35-2.78 m. Below this layer of siltstone and sandstone lies the \#9 coal seam with a thickness of $0-4.4 \mathrm{~m}$. The \#13, \#12 and \#10 coal seams have been mined out, but the \#9 coal seam was currently being mined using the longwall mining method. All of coal specimens for this work were collected from the \#9 coal seam. The measured coal stratum was composed of $56.40 \%$ are nauseous shale, $26.18 \%$ fine sandstone, $5.81 \%$ coal and $11.61 \%$ metamorphic rock. The locations of mine

C. Wang, Evolution, Monitoring and Predicting Models of Rockburst, 


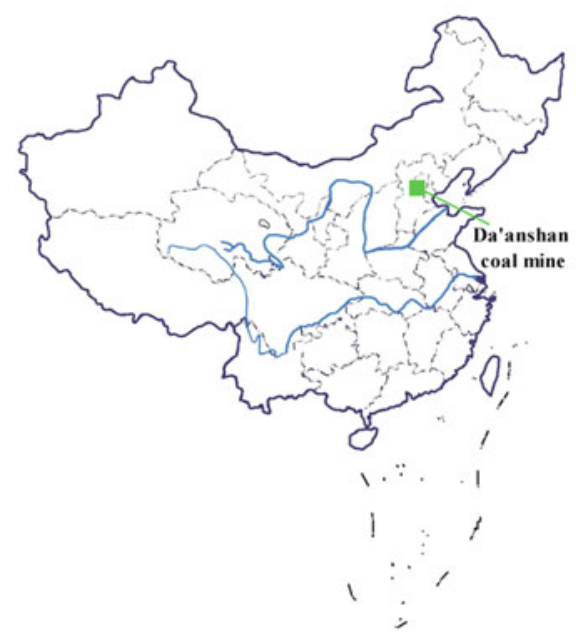

(a) Location of the coal mine.

\begin{tabular}{|c|c|c|c|}
\hline $\begin{array}{c}\text { Thickness } \\
\text { (m) }\end{array}$ & Column & Lithology & $\begin{array}{l}\text { Geological } \\
\text { descriptions }\end{array}$ \\
\hline $0.77-2.53$ & & \multirow{2}{*}{ Coal $\# 13$} & \multirow{2}{*}{$\begin{array}{c}\text { Medium hard, semibright. } \\
\text { unstable }\end{array}$} \\
\hline 1.5 & & & \\
\hline $0.1-0.2$ & & Sandy conglemerate & Asth Black, flise-grained filling \\
\hline $0.64-2.41$ & & \multirow[b]{2}{*}{ Coal $\# 12$} & \multirow{2}{*}{$\begin{array}{c}\text { Medium hard, semibright, } \\
\text { unstable }\end{array}$} \\
\hline 1.3 & & & \\
\hline$\frac{0.65-1.14}{0.8}$ & & Siltstone, sandstone & $\begin{array}{l}\text { Ash Black, argillaceous } \\
\text { cement }\end{array}$ \\
\hline $0-7.41$ & & \multirow[b]{2}{*}{ Coal in 10} & \multirow{2}{*}{$\begin{array}{l}\text { Medium hard, unstable from } \\
\text { semidull to semibright }\end{array}$} \\
\hline 1.42 & & & \\
\hline$\frac{1.35-2.78}{1.7}$ & 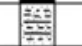 & Siltstone, sandstone & $\begin{array}{c}\text { Ash black, argillaceous } \\
\text { cement }\end{array}$ \\
\hline $\begin{array}{c}0-4.40 \\
1.76 \\
\end{array}$ & & Coal if 9 & $\begin{array}{l}\text { Medium hard, dirt band } \\
\text { semibright, unstable }\end{array}$ \\
\hline$\frac{3.15-6.18}{4.5}$ & 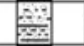 & Siltstone, sandstone & $\begin{array}{l}\text { Ash black, arpillaceous } \\
\text { cement }\end{array}$ \\
\hline $0.83-6.08$ & & \multirow[b]{2}{*}{ Coal \& 7} & \multirow{2}{*}{$\begin{array}{l}\text { Medium hard, semibrighth. } \\
\text { unstable }\end{array}$} \\
\hline 2.44 & & & \\
\hline$\frac{1.02-3.2}{1.7}$ & F & Sandstone & $\begin{array}{c}\text { Ash black, argillaceous } \\
\text { cement }\end{array}$ \\
\hline $0-10.63$ & & \multirow[b]{2}{*}{ Coal 116} & \multirow{2}{*}{$\begin{array}{c}\text { Medium hard, estremely } \\
\text { unstable, from semidull to } \\
\text { semibrizht }\end{array}$} \\
\hline 1.05 & & & \\
\hline $1.5-5.8$ & \multirow{2}{*}{ 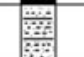 } & \multirow{2}{*}{ Siltstone, sandstone } & \multirow{2}{*}{$\begin{array}{c}\text { Ash Dlack, argillaceous } \\
\text { cement }\end{array}$} \\
\hline 4.2 & & & \\
\hline
\end{tabular}

(b) Geologic column.

Fig. 2.1 Location of Da'anshan coal mine and the geologic column of coal seams

and the coal core zones associated with the geological structures in the Da'anshan coal mine are shown in Fig. 2.1.

The rock samples were got from the roof of Xiexhuang mine coal seam, and the roof is mainly limestone. Xiezhuang coal mine is located in the South-West of Xinwen coalfield, $13 \mathrm{~km}$ east of Xintai City, mining depth +110 to $-1050 \mathrm{~m}$, as shown in Fig. 2.2. The mine is $9-12 \mathrm{~km}$ long from east to west and $1.1-4.2 \mathrm{~km}$ from north to south, with an area of about $41.5109 \mathrm{~km}^{2}$. Geographic coordinates: longitude $117^{\circ} 31^{\prime} 03^{\prime \prime}-117^{\circ} 41^{\prime} 15^{\prime \prime}$, latitude $35^{\circ} 53^{\prime} 45^{\prime \prime}-35^{\circ} 56^{\prime} 58^{\prime \prime}$. East-west length 9-12 km, north-south width of $1.1-4.2 \mathrm{~km}$, an area of about $41.5109 \mathrm{~km}^{2}$. Geographic coordinates: longitude $117^{\circ} 31^{\prime} 03^{\prime \prime}-117^{\circ} 41^{\prime} 15^{\prime \prime}$, latitude $35^{\circ} 53^{\prime} 45^{\prime \prime}-35^{\circ}$ $56^{\prime} 58^{\prime \prime}$. The geological structure of mining area is a northward inclined monoclinic structure, and the subordinate folds are not developed. Coal-bearing strata is a Carboniferous Permian, is toward the east and west, which is a monoclinic structure with a tendency of nearly $6^{\circ}-36^{\circ}$. It belongs to the Permian Carboniferous land and sea mutual coal deposits in North China. The total thickness of coal seams is $256 \mathrm{~m}$, including 19 coal seams, the average thickness of coal seams is $12.07 \mathrm{~m}$, the total thickness of recoverable coal seams is $10.34 \mathrm{~m}$, and the coal content coefficient is $4 \%$. 


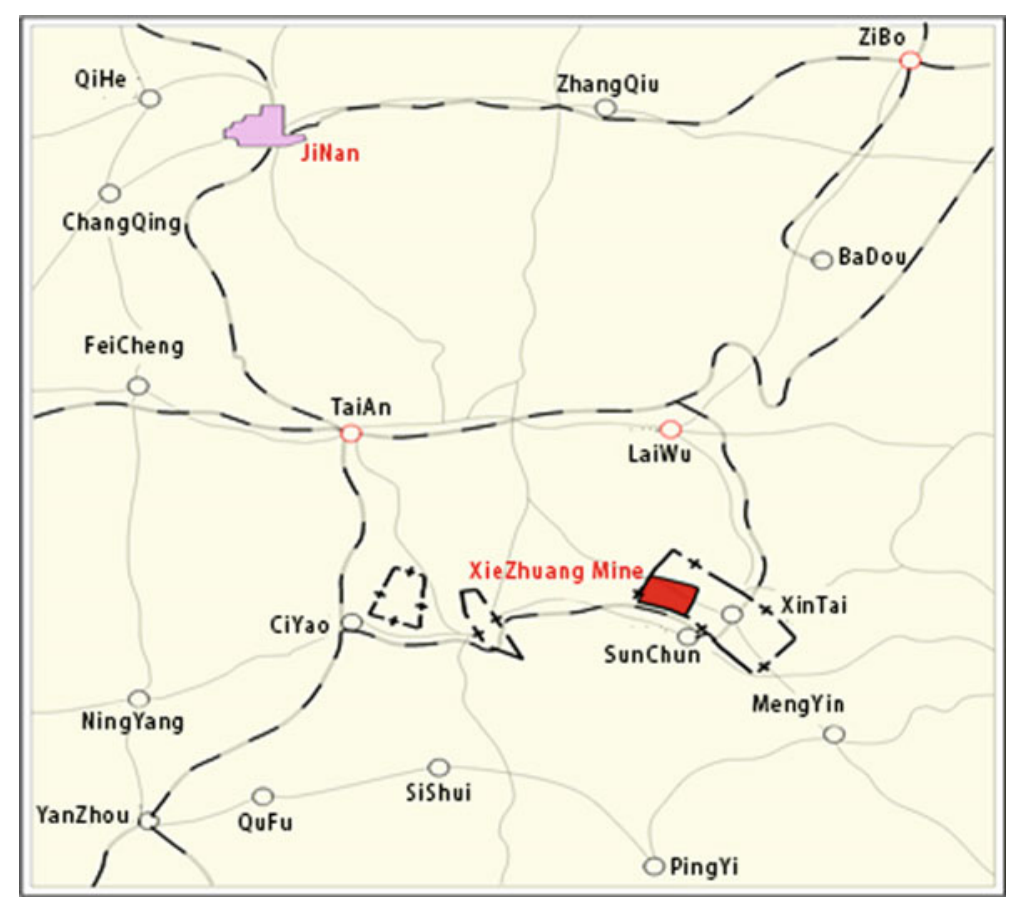

Fig. 2.2 The location of Xiezhuang coal mine

\subsection{Experimental Equipments}

\subsubsection{Laboratory Equipments}

\section{Servo Stiffness Compressor}

The specimens were loaded by a microcomputer-controlled electrohydraulic servo stiffness compressor (GAW-2000, Chaoyang Test Instrument CO., LTD, Changchun, China). As shown in Fig. 2.3, the compressor can perform tensile test, uniaxial compression test and traditional triaxial test. The computer that connected with the compressor directly controlled indenters; host computer recorded the test data accurately and draw the stress-strain curve of samples. These equipments were used to monitor the failure of specimens immediately and dynamically. After the test, the collected stress and strain data were processed and analysed by electrohydraulic servo stiffness compressor, then we obtained the mechanical parameters of rock and the stress-strain curve during loading.

2. AE Monitoring System

The AE monitoring system equipment includes the PCI-2 fully digital AE signal collection, and the analysis system of Physical Acoustics Company (PAC CO., 


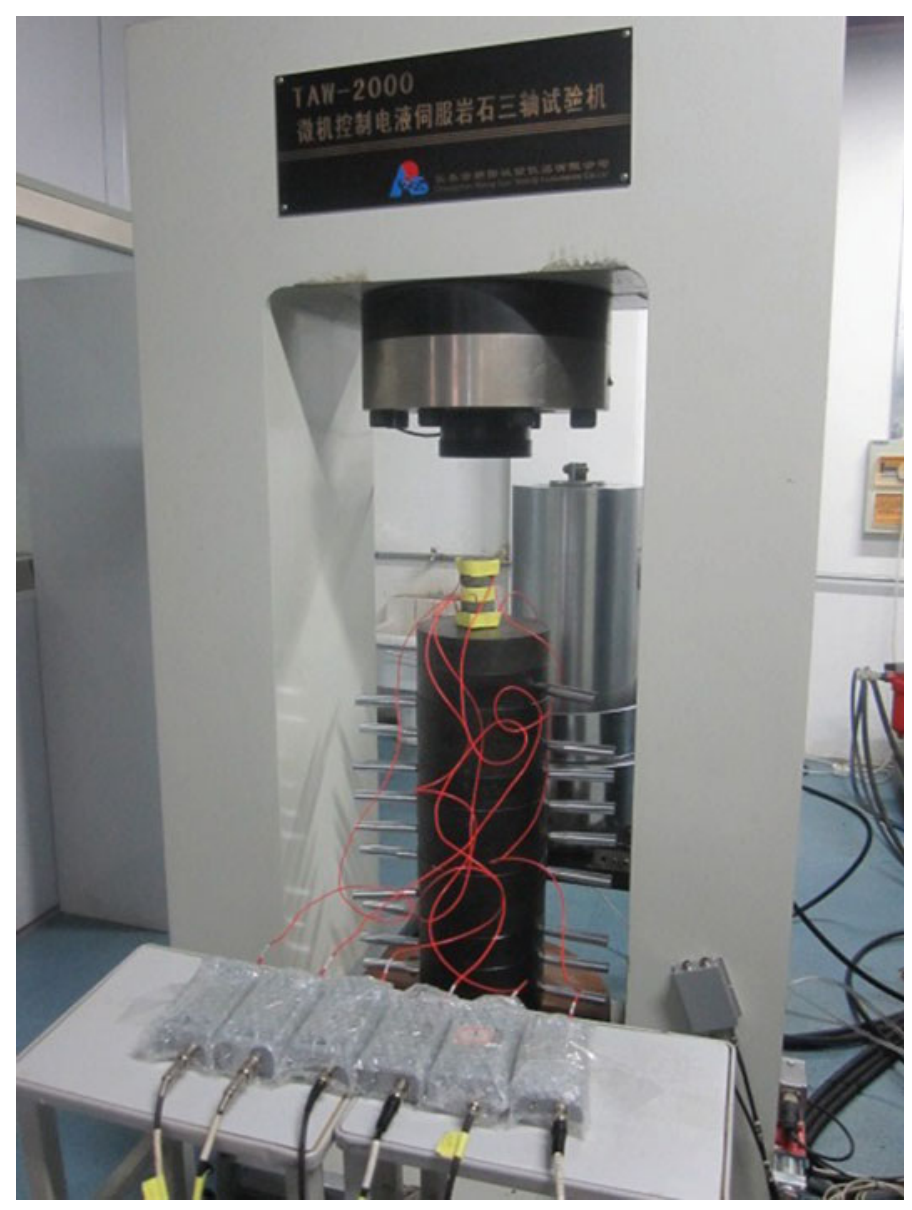

Fig. 2.3 TAW-2000 computer control electro-hydraulic servo rock triaxial testing machine

New Jersey, USA). As shown in Fig. 2.4, the system has 6 collection channels, including sensor, preamplifier, host, and processing softwares to monitor, collect and store AE signal immediately. It displays the dynamic changes of related parameters on the computer monitor connected with it.

The micro sensors, with a frequency of $300 \mathrm{kHz}$, can obtain AE events information accurately. Because of small size sensor, the perception ability range is narrow. Therefore, it is only suitable for specimens with relatively small scales. The preamplifier has an automatic testing function with a frequency range of 20$1200 \mathrm{kHz}$, the preamplifier has three different level, 20, 40 and $60 \mathrm{~dB}$ respectively, can adapt to different test conditions and requirements. The processing card of $\mathrm{AE}$ collection system (PCI-II) is larger range, from $1 \mathrm{kHz}$ to $3 \mathrm{MHz}$, and has conversion rate of 18 bit A/D. The system has 6 collection channels, which can guarantee the acquisition and processing of signals. Meanwhile, the 6 channels can 


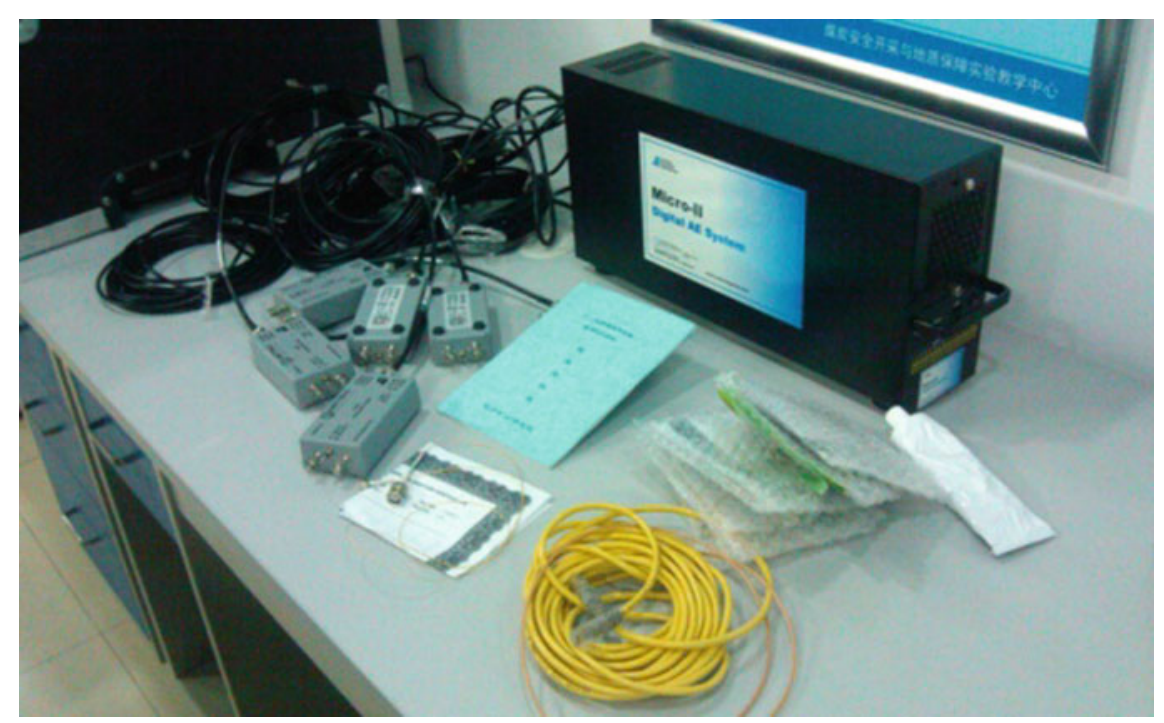

Fig. 2.4 PCI-II AE testing system

also realize the three-dimensional positioning of AE events. The processing software of this system can realize the acquisition and storage of AE signals immediately, and can replay the whole process of AE signal collection, export various AE parameters. AE signal source can also be automatically located by this system (one-dimensional line location, two-dimensional area localization and three-dimensional spatial localization).

\section{Infrared Thermal Imager}

The uncooled infrared focal plane thermal imager (InfRec R300) was used to detect the infrared radiation temperature field of rock samples. This instrument, manufactured by Nec Corp, was a temperature accuracy of 0.05 centigrade, a spatial resolution of $1.2 \mathrm{rad}$, and a resolution of $320 \times 240$ pixels. The temperature range of InfRec R300 is from 10 to 40 centigrade, and the highest graphic recording rate was 60 frames per second. The working spectrum of thermal imager cameras ranged from 8 to $14 \mu \mathrm{M}$, could measure surface temperatures in the $8-14 \mu \mathrm{m}$ infrared atmospheric window. The decay of radiation signal received was mildly spread through air in the continuous wave length $(8-14 \mathrm{~m})$. Therefore, it has a potential advantage over the accuracy of temperature detection. Thermography data is stored in InfRec R300 in a time series and can be analyzed by the attached NS 9500 software, as shown in Fig. 2.5.

\section{CT Test Scanning Equipment}

As shown in Fig. 2.6, X-ray industrial CT test inspection system (ACTIS300-320/ 225, Bio-Imaging Research, Inc. Lincolnshinre, Lllinois, United States of America). 


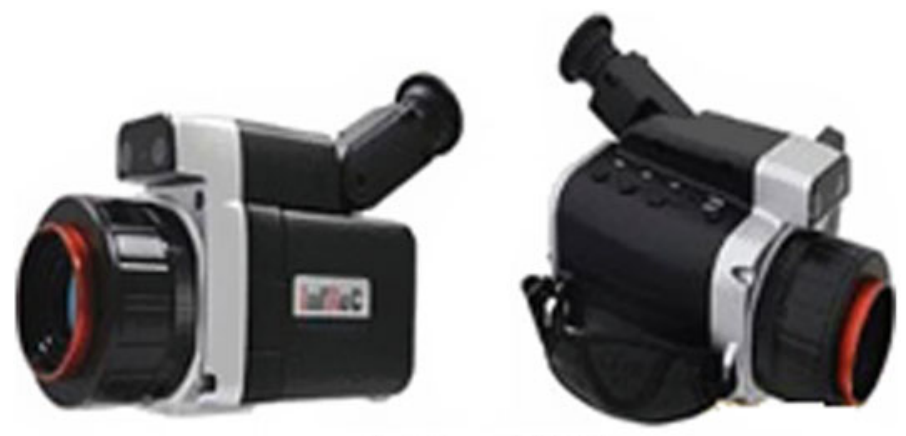

InfRec R300

Fig. 2.5 InfRec R300 thermal infrared imager

The system has advantages of 3D reconstruction and high precision image acquisition on large size samples. The obtained images using industrial CT with a high energy X-ray source are high density and spatial resolution. The resolution of industrial CT scanning system is $0.5 \mathrm{~mm} \times 0.35 \mathrm{~mm} \times 1 \mathrm{~mm}$. The radiation penetrates the surface of rock sample, the different density in the rock sample can be used to distinguish the different substances, and the energy absorbed by different materials is also different. Therefore, images with different shades were displayed centrally on the data acquisition panel behind the rock sample, rock samples were rotated at constant speed, and were collected and scanned continuously. The system was carried out full range data acquisition and continuous scanning of rock samples, 3D model of rock samples was obtained by reconstruction with the software MIMICS. To carry out more detailed post analysis, we need to segment the 3D model into slices and store it as a two-dimensional bin file, and then convert it into a picture format file through MATLAB software programming. The X-ray classification depends on the size and lithology of rock being tested; this system can be applied to nondestructive testing of metal or nonmetal materials with of different densities. The advantages are nondestructive, accurate and intuitive. In addition, for the undisturbed testing scanning of rock and soil engineering materials, the inhomogeneous distribution characteristics of rock materials and their internal structures were obtained from the scanning images, and the three-dimensional structures of rock samples were accurately restored. The samples were non-disturbance test, microscopic physical and mechanical properties of rock were described, such as the change of density distribution; crack size; the rock impurities and distribution etc. 


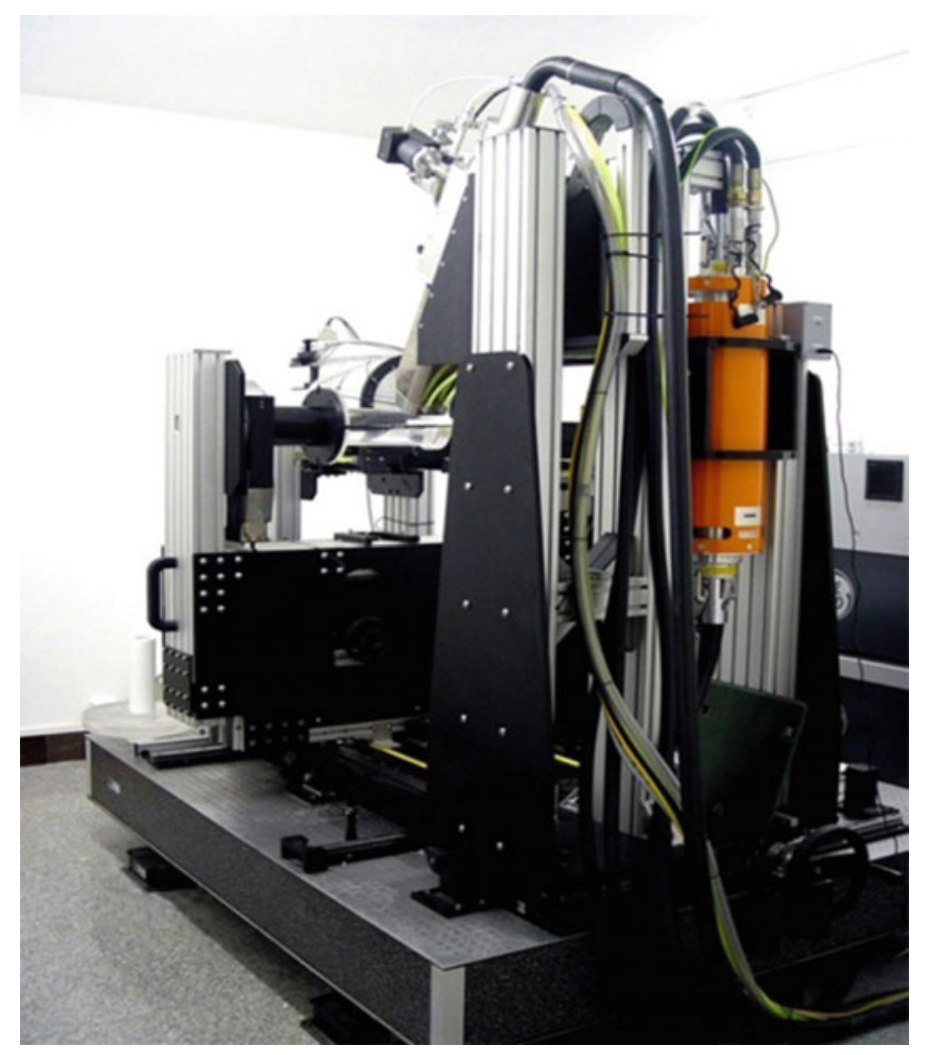

Fig. 2.6 ACTIS300-320/225 X-ray industrial CT testing system

\subsubsection{Field Equipment}

Field test equipment is a microseismic monitoring system for Huize Lead-Zinc mine in China. In August 2007, a digital 24-channel MS monitoring system of IMS Company in South African, which consisted of 12 sensors and 4 QS seismic data acquisition instruments, was established to monitor the MS events in deep mining, as shown in Fig. 2.7. Then, the monitoring system was designed, installed, debugged. The monitoring system consists of four parts: surface monitoring station, underground data exchange center, signal acquisitions and sensors. The surface monitoring station was located in the mine pit office building. The underground data exchange center was located in the middle of $1331 \mathrm{~m}$ vertical shaft. The sensors were arranged in the surrounding rockmass of monitoring area.

The microseismic monitoring system was divided into two parts: surface and subsurface, they were both long-term monitoring service facilities. The underground data exchange center was located in the $2 \#$ shaft portal signal chamber of middle 


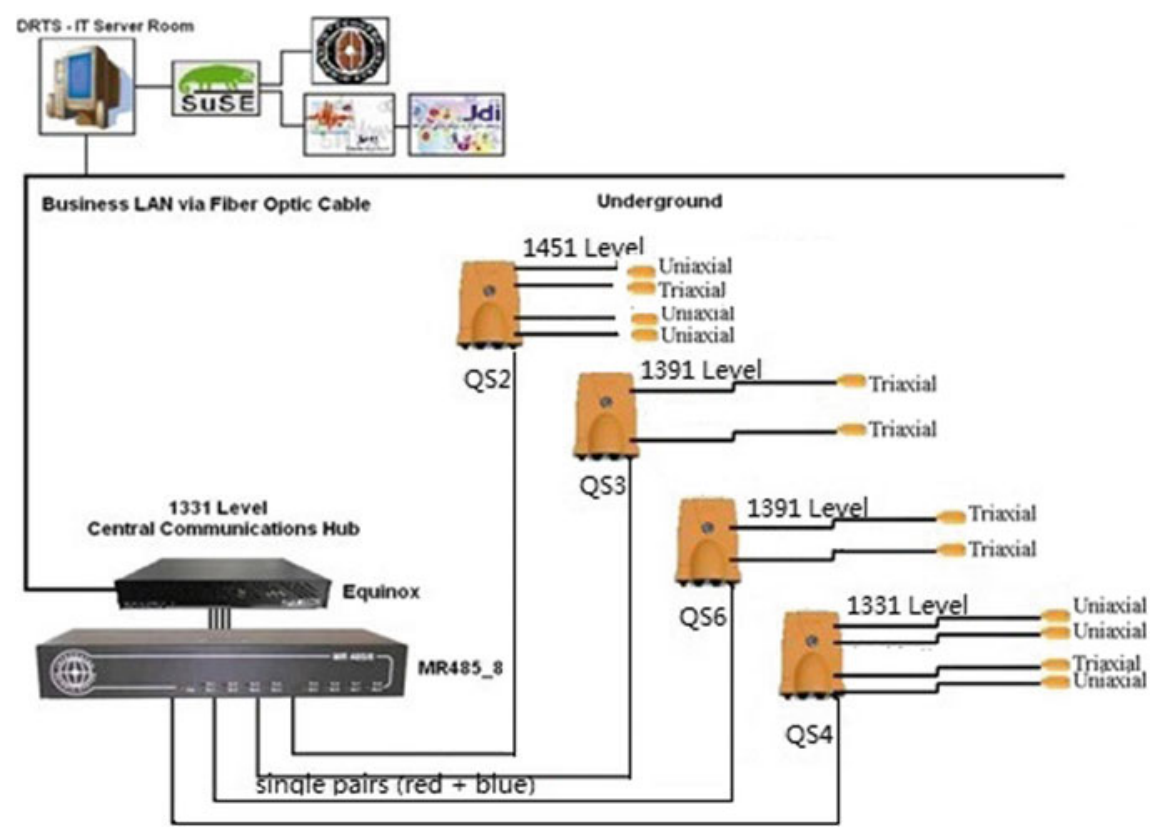

Fig. 2.7 Microseismic monitoring system in Huize mine (after Wang 2013)

section of $1331 \mathrm{~m}$, signal spread to the surface monitoring station through the optical fiber cables, these sensors and the underground data exchanger communicated with the signal cables.

\subsection{SEM and EDS}

Field emission scanning electron microscopy (SEM) is an electronic optical instrument based on modern matter wave theory. In fact, SEM uses a focused high-energy electron beam to observe and scan a sample, thus obtaining a variety of physical information about the samples. Through the acceptance, amplification and display imaging of specific region information of target sample, the surface topography characteristics of sample are observed. Now, this technique has been widely used in micro mechanics research. SEM can be used to observe the metal, ceramics, semiconductors, minerals, and nanoscale one-dimensional, two-dimensional, three-dimensional materials surface and fracture appearance, and the microstructure, size analysis, microstructure and phase distribution of nanomaterials. Compared with optical microscopy, scanning electron microscopy has the advantages of simple sample preparation, large depth of field, and wide adjustable range of amplification coefficient, large field of view and high frequency of acquisition. 
Energy Dispersive Spectrometer (EDS) is used to analyze component elements, species and their contents of sample, and used in conjunction with scanning electron microscopy and transmission electron microscopy. Since each element has a characteristic wavelength range of X-ray, the energy spectrometer can be used to analyze the composition of material. The spectrometer can be used to analyze the composition and element distribution of Be-U elements of various powders and solid blocks with point, line and surface analysis function, the surface distribution is only $1 \mathrm{~min}$, the scanning speed is quick.

The scanning test was conducted at the Institute of Process Research, Chinese Academy of Sciences. As shown in Fig. 2.8, scanning electron microscope (JSM-7001F) produced by Japan Electronics Corporation and X-MAX energy spectrum analyzer produced by Oxford Instrument Company. The technical parameters are as follows:

(1) Scanning electron microscope resolution: $2 \mathrm{~nm}(30 \mathrm{kV}) / 3.0 \mathrm{~nm}(1 \mathrm{kV})$; acceleration voltage: $0.5-30 \mathrm{kV}$; magnification: $10-500 \mathrm{~K}$.

(2) The immersion thermal field electron gun has the highest beam intensity of $200 \mathrm{nA}$, and has the characteristics of large beam and high resolution.

(3) The automatic diaphragm angle controller does not need to adjust the aperture; fully automatic control of focusing, closing the axis, eliminating astigmatism, and controlling the scanning speed.

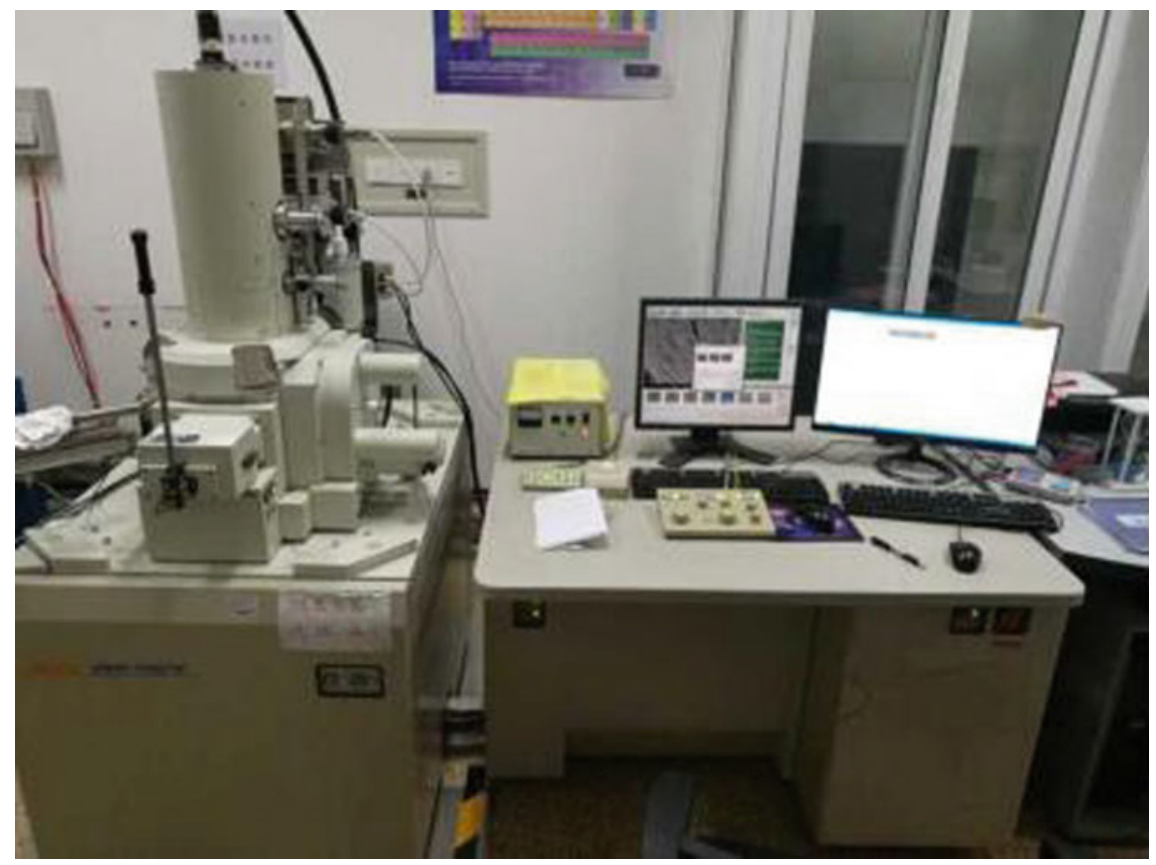

Fig. 2.8 Field emission scanning electron microscope and energy spectrum analyzer 
(4) The design of magnetic leakage is convenient for the observation of magnetic samples, and is suitable for cooperating with a variety of analytical probes.

(5) The effective area of spectrum probe is $50 \mathrm{~mm}^{2}$, Input count rate $>500,000 \mathrm{cps}$, Acquisition count rate $>200,000 \mathrm{cps}$, the Typical resolution of $\mathrm{Mn} \mathrm{K}<125 \mathrm{eV}$, $\mathrm{C} \mathrm{K} \alpha<48 \mathrm{eV}$.

Figure 2.9 shows SEM images of a sample at magnifications of 200 times, 500 times, 1000 times and 10,000 times. Figure 2.10 depicts the spectrogram, and Table 2.1 shows the chemical composition of samples.

Figure 2.11 shows the SEM image at magnification of 200 times, 1000 times and 2000 times of coal specimens. Figure 2.12 depicts the spectrogram of specimens and Table 2.2 shows the chemical component of coal specimens. It is shown by SEM observation that the coal specimen is a heterogeneous porous material. It is readily found that the rock heterogeneity is attributed to the existence of minerals of different hardness, micro cracks, and micro-cavities.

(a)

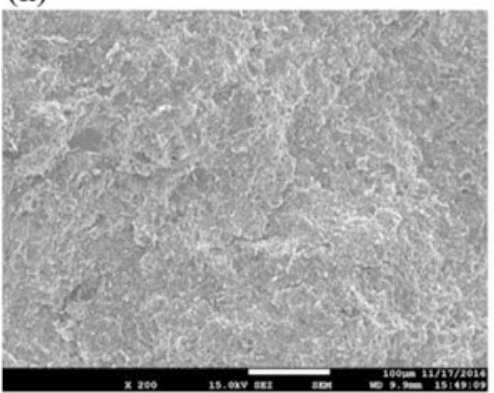

(c)

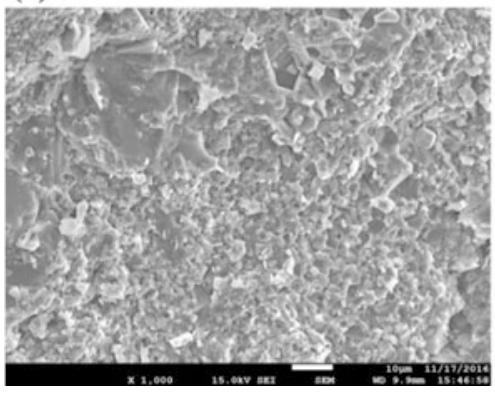

(b)

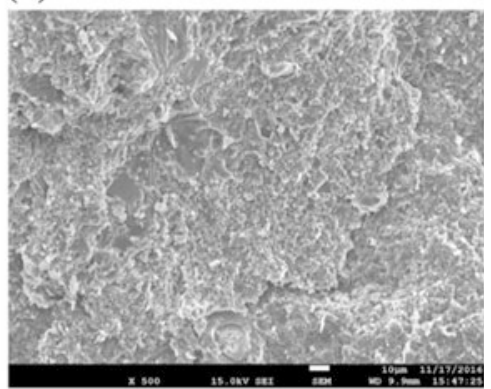

(d)

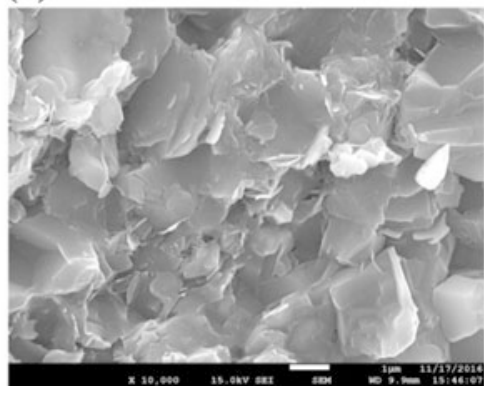

Fig. 2.9 SEM image at magnification of sample 
(a)

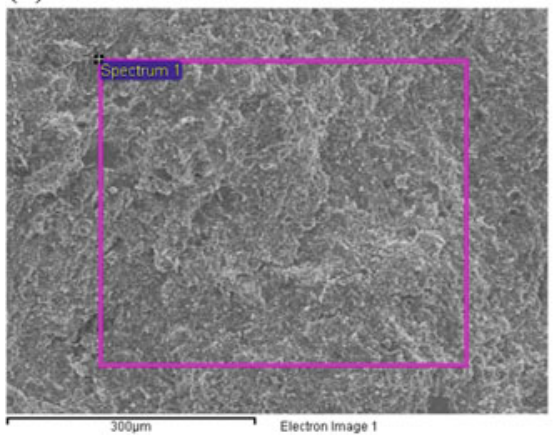

(b)

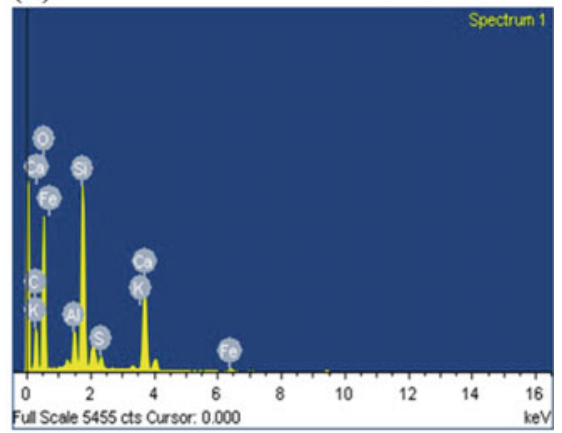

Fig. 2.10 The spectrogram of sample

Table 2.1 Chemical components of samples

\begin{tabular}{l|l|l|l}
\hline Chemical elements & Percentage of weight & Percentage of atoms & Chemical formulas \\
\hline $\mathrm{C}$ & 16.86 & 26.07 & $\mathrm{CaCO}_{3}$ \\
\hline $\mathrm{O}$ & 45.84 & 53.22 & $\mathrm{SiO}_{2}$ \\
\hline $\mathrm{Al}$ & 2.59 & 1.78 & $\mathrm{Al}_{2} \mathrm{O}_{3}$ \\
\hline $\mathrm{Si}$ & 14.67 & 9.70 & $\mathrm{SiO}_{2}$ \\
\hline $\mathrm{S}$ & 1.50 & 0.87 & $\mathrm{FeS}_{2}$ \\
\hline $\mathrm{K}$ & 0.68 & 0.32 & \\
\hline $\mathrm{Ca}$ & 15.99 & 7.41 & \\
\hline $\mathrm{Fe}$ & 1.87 & 0.62 & $\mathrm{Fe}$
\end{tabular}

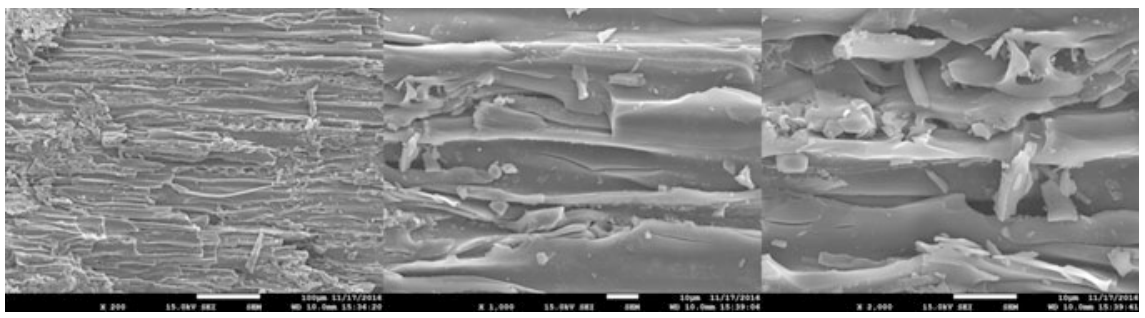

Fig. 2.11 SEM image at magnification of coal specimens 

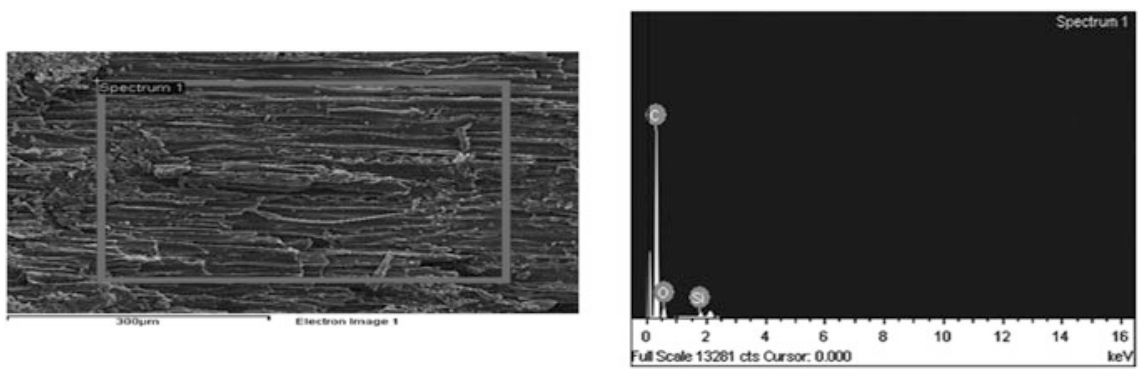

Fig. 2.12 The spectrogram of coal specimens

Table 2.2 Chemical components of coal specimens

\begin{tabular}{l|l|l|l}
\hline Chemical elements & Percentage of weight & Percentage of atoms & Chemical formulas \\
\hline C K & 83.76 & 87.68 & $\mathrm{CaCO}_{3}$ \\
\hline O K & 14.95 & 11.75 & $\mathrm{SiO}_{2}$ \\
\hline Si K & 1.28 & 0.57 & $\mathrm{SiO}_{2}$ \\
\hline
\end{tabular}

\section{Reference}

Wang CL (2013) Identification and early warning method for rock mass instability. Disaster Adv 6 (12):101-105

Open Access This chapter is licensed under the terms of the Creative Commons Attribution-NonCommercial 4.0 International License (http://creativecommons.org/licenses/by-nc/ $4.0 /$ ), which permits any noncommercial use, sharing, adaptation, distribution and reproduction in any medium or format, as long as you give appropriate credit to the original author(s) and the source, provide a link to the Creative Commons license and indicate if changes were made.

The images or other third party material in this book are included in the book's Creative Commons license, unless indicated otherwise in a credit line to the material. If material is not included in the book's Creative Commons license and your intended use is not permitted by statutory regulation or exceeds the permitted use, you will need to obtain permission directly from the copyright holder.

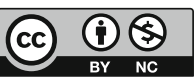




\section{Chapter 3 \\ Mechanism and Predicting Theory-Based of Rockburst Evolution}

\subsection{Introduction}

The dynamic hazard, caused by failure of surrounding rockmass because of rock excavation, is the most direct danger of mining safety and efficient production. Many scholars (Wang et al. 2014, 2015; Lindin and Lobanova 2013; Chen et al. 2015; He et al. 2010; Pan et al. 2014) paid much attention to it. Although many achievements were be made in theoretical and field cases, there is no unified understanding of rock dynamic hazards, especially the mechanism and predicting of rockburst, and further research is needed. Previous researches on rock failure and rockburst induced conditions have been carried out, but the mechanism and predicting of rockburst is complicated, and there is no mechanism and prediction model to explain the occurrence of rockburst satisfactorily. Therefore, the study of predicting rockburst induced conditions and the failure behavior of rock fracture is analyzed by proper theory. Most of these can provide reasonable suggestions for mining design; mining method selection and rockburst hazard prevention measures. It is of great significance for mine safety and high efficiency mining. In complex mining conditions, in order to solve the key technical problems of predicting rockburst, first of all, it is necessary to analyze the conditions induced rockburst, so as to lay the foundation for predicting of rockburst. The essence of rock failure is the release of elastic strain energy stored in rock under the action of stress. Therefore, one of key factors to study rock failure is the stress distribution in rock and the relation between rock elastic energy and dissipation energy per unit volume. If most of energy absorbed by rock is dissipated during loading, then the final failure mode of rock will be static. On the contrary, it is dynamic.

On the basis of these, combined with the deformation characteristics of rocks, the author explored the stress-energy mechanism of rockburst. Since the 1970s, the emergence of nonlinear theory greatly promoted the development of rock mechanics such as mutation theory, dissipative structure theory, fractal structure theory etc. Using these theories to study rock failure and rockburst, it not only 
correctly described its nonlinear dynamic characteristics, but also developed new concepts, new theories and methods for predicting rockburst.

In this chapter, the rockburst induced mechanism, rockburst predicting dynamic theory and rockburst stress-energy process were introduced in detail, and new predicting models and methods were established based on these theories. Experimental and field investigations showed that the application of these methods achieved the desired results, but also provided a new method for rockburst predicting.

\subsection{Mechanism of Rockburst}

Rockburst is generally considered to be induced by mining and accompanied by microseismic activity and elastic energy release. The excavation will disturb the original rock stress field. When the stress redistribution causes the surrounding rockmass to reach the critical state of destruction, some external factors, such as blasting stress wave and so on, may cause severe damage to the surrounding rockmass. In mechanism, rockburst is characterized by its occurrence and dynamic process compared with statics. But compared with dynamics, the evolution process of rockburst is quasi-static. It is a mechanical process from static deformation to loss of stability, and a result that rockmass structure loses its bearing capacity gradually and its stability finally. Therefore, the sufficient elastic strain energy and the stress concentration place are two main conditions to induce rockburst.

The released elastic strain energy stores in rockmass is the evolution process of rockburst. The key of rock failure is the internal stress distribution and the unit volume of rock elastic energy dissipation, and most of energy absorbed by rock is dissipated during loading, then the final failure mode of rock will be dominated by static. On the other hand, if the energy dissipation is less during the loading, rock will destroy more strong eventually, and some elastic energy will also be converted into the kinetic energy. Cook (1966) proposed the energy theory of rockburst in 1960s. It was pointed out that rockburst occurs when the mechanical equilibrium state of surrounding rockmass system was destroyed, and the energy released was greater than the energy consumed. In the 1970s, Brauner (1994) proposed the energy rate theory:

$$
\alpha\left(\frac{\mathrm{d} E_{R}}{\mathrm{~d} t}\right)+\beta\left(\frac{\mathrm{d} E_{E}}{\mathrm{~d} t}\right)>\frac{\mathrm{d} E_{D}}{\mathrm{~d} t}
$$

$\alpha$ is effective coefficient of surrounding rockmass energy release, $\beta$ is effective coefficient of energy release of orebody, $E_{R}$ is the energy stored by surrounding rockmass, $E_{E}$ is energy stored by orebodies, $E_{D}$ is energy consumed at the junction of orebodies and surrounding rockmasses. Energy theory, based on the law of 
conservation of energy, breaks the limitations of traditional theory and answers the question of energy source of rockburst.

From the point of stress concentration, the main idea is that once the stress on rock exceeds its limit of strength, rock begins to break. The early researchs took the surrounding rockmass system as the object, and considered that the key to rockburst was the strength of surrounding rockmass system. Later, people gradually realized that the decisive factors that lead to rockburst are not only related to the strength of surrounding rockmass system, but also to the stress environment, mineral composition and excavation activities of rockmass. Under uniaxial loading, one of necessary conditions for rockburst is that the stress is greater than the compressive strength of rock. Under the triaxial loading condition, when the stress in the two directions is constant, the load in the third directions is gradually reduced. The stress value of rockburst was lower than that of rock (He et al. 2010). Therefore, according to the stress condition of rockburst, the method of reducing stress concentration is often adopted to prevent rockburst. If there is no necessary control for the surrounding rockmass, the elastic strain energy of surrounding rockmass storage will be converted to the kinetic energy of broken rockmass, which may lead to rockburst.

\subsection{Mechanism of Rockburst Stress-Energy Evolution}

Rockburst is a nonlinear dynamic process. In the course of its occurrence, part of energy is transformed into kinetic energy of broken rock blocks, and the broken rock blocks are ejected. Therefore, from the energy point of view, rock in compression deformation stage must accumulate enough elastic strain energy, in order to ensure more energy transfer to the kinetic energy of rock failure. The energy condition of rockburst is that the elastic strain energy accumulated in the process of rock compression is much larger than the energy consumed.

Rockburst is essentially a rapid release process of elastic strain energy accumulated in the rockmass. The main factors that affect the occurrence of rockburst is actually the impact of rockmass into a high energy storage factor, high energy rockmass are more likely to rockburst, and become high energy rockmass includes two aspects, that is to say, rockmass had sufficient capacity to store large elastic strain energy and a high concentration of stress (Zhao 2015). The conditions for the occurrence of rockburst propagation are as follows: a high concentration stress status and a large amount of releasable strain energy. Based on these two conditions, the mechanism of stress-energy evolution in rock is discussed.

Because of initial damage of rockmass, the density changes for this reason. Thus, the expansion of microhole and microcrack, which are caused by loading, are called positive damage. Similarly, the closure of microcracks and micropores is called negative damage. According to the basic principle of damage mechanics and rock mechanics, the pre-peak stage of uniaxial static loading of rock is regarded as the stage of rockburst evolution. From the energy point of view, the energy evolution of 


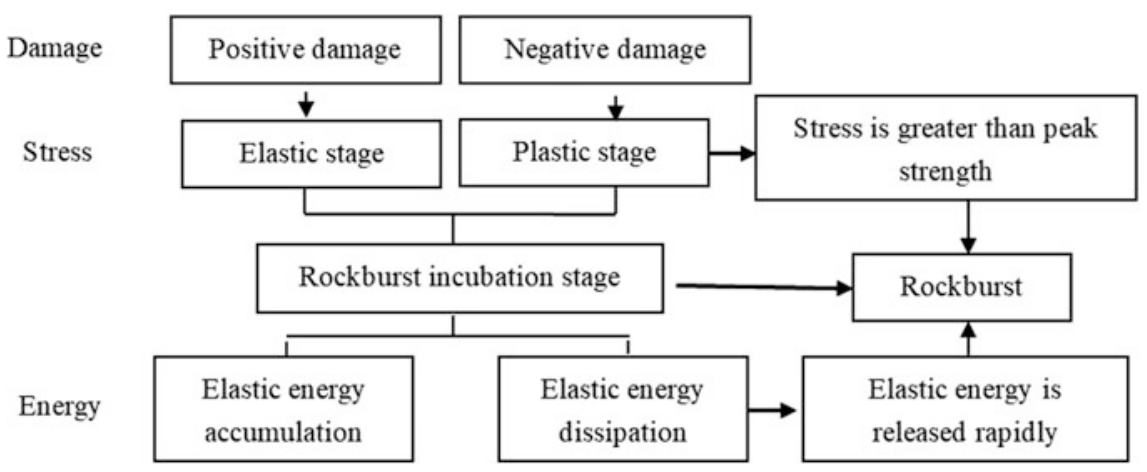

Fig. 3.1 The diagram of stress-energy mechanism of rockburst preparation and development

rockburst is mainly divided into two stages: energy accumulation and energy dissipation. The occurrence of rockburst is energy release. From the stress point of view, rockburst occurs at the pre-peak stage, while rockburst occurs at the post peak stage, as shown in Fig. 3.1.

In the initial stage of rock deformation, rock stress is small, the stress distribution is relatively uniform, the micro-cracks are compacted, the rock structure units are in the elastic stage, the elastic strain can begin to accumulate and rockburst is at the incubation stage. In addition, due to the micro-crack closure, the macro damage variable becomes smaller, negative damage occurs, rock mechanical properties are strengthened, the elastic modulus becomes larger, and the energy storage capacity of rock is improved.

Rock has passed an initial stage of compressive deformation and enters elastic stage. With the increase of stress, most of structural units are still in a stable status, and the elastic strain can begin to accumulate in large quantities, which is the key stage of rockburst evolution. This moment the microcracks are basically compacted and the damage cracking is not obvious. However, in the middle and late stages of the elastic stage, due to the increase of stress and the material properties lead to stress concentration part of structural unit, rock stress exceeds its compressive strength. The structural units lose its load-bearing capacity, and then the macroscopic damage of rock changes from negative damage to positive damage and the elastic modulus starts to decrease and the elastic strain energy begins to dissipate.

As the load continues to increase, rockmass enters the plastic deformation stage. As a result of material composition and structure of rockmass, the plastic phase of rockmass generally is relatively short, and some rocks even destroy directly during the elastic stage. In the plastic stage, a large number of rock units are destroyed because of increase of stress. The damage cracking is aggravated, the microcracks are expanded rapidly, and the macro fracture cracks are formed. The strain energy dissipation is increased, and the damage variable increases rapidly. At this point, rock is in the critical state of rockburst occured. When rock failure criterion is 
reached and sufficient strain energy is released, rockburst may occur at any moment.

In this chapter, the mechanism of rockburst stress-energy evolution is obtained by analyzing the pre-peak stage of rock compressive deformation: rockburst, as a way of rock fracture, is mainly caused by the accumulation of stress and the accumulation release of elastic strain. The change of stress for rockburst occurred plays a leading role, not only it leads to the accumulation and release of elastic strain energy, but also the damage cracking unit of rock structure. Thereby, reducing the energy storage capacity of rock elastic strain energy. In addition, the elastic strain energy released by rockburst is the result of accumulation and dissipation of elastic strain energy.

\subsection{Nonlinear Dynamic Theory of Predicting Rockburst}

\subsubsection{Mutation Theory}

The stable and unstable statuses referred to the theory of mutation are relative to certain control conditions. Under the control of external forces, the stable and unstable status of system can be freely transformed. If the initial state of system is stable, the status of system will change as a result of continuous control force. When the control factor reaches a certain limit (critical value), the status of system has reached the critical status of stability and instability. Although the control factor no longer changes, the status of system remains rapidly away from the critical value, changing to another stable state rapidly and this is the mutation. When the control factor reaches the critical value, the mutation does not occur. However, the mutation was occured within a certain period of time after the critical value (Shen 2006). When the specimens are subjected to external loads, the internal microcracks continue to develop, expand, merge and coalescence and the damage keep accumulating. When the internal damage accumulates to a certain extent, rock are in the critical state and the system tends to be unstable, a little disturbance of stress will lead to a sudden change of rock status. The macroscopic behavior is sudden failure of rock. Mutation theory, which is a nonlinear theory that studies the phenomenon of sudden change, can solve such problems very well.

Through AE testing of specimen loading, the damage was described using the mutation theory; the characteristics during the evolution of rock damage were obtained. In this chapter, the cumulative damage instability and the precursory characteristics of rock failure were analyzed. 


\subsubsection{Damage Theory}

Damage theory is a new branch of mechanics developed around 1970s. Kachanov (1958) studied the creep failure of metals, the concept of continuity factor and effective stress were proposed to study the influence of internal defects on the deformation and failure of metals. Later, Robotnov (1969) introduced the concept of damage factor, and they did pioneering work for the establishment and development of damage mechanics. Lemaitre (1974) applied the concept of damage to low cycle fatigue. In 1974, British scholar Leckie (1974) and Swedish scholar Hult (1975) promoted the development of damage theory in the study of creep. Using the theory of continuum mechanics, many scholars were regard damage factor (DF) as a field variable and called it a damage variable. Thus, the basic frame and foundation of damage mechanics were formed.

Damage mechanics can establish damage evolution equation by defining specific damage variables. Therefore, the effects of damage on rock deformation characteristics can be reflected, especially for nonlinear dynamic processes such as rockburst. The damage theory was used to describe the physical processes of rockbursts (Sato et al. 1986). Under the action of external force disturbance, tensile stress status of local damage appeared in the rockmass (Leighton 1982). When the stress exceeds certain ultimate strength, new microcracks were formed in the rockmass. With the increase of external force, a large number of microcracks were gathered in some weak areas of rockmass, which was called the serious damage zone (Ma et al. 2016). In the evolution of rockburst damage, many scholars established a variety of macroscopic damage models.

In the process of loading, AE events were closely related to the interaction and propagation process of microcracks. Therefore, AE events were consistent with the damage of specimen. The pattern of rock failure change was analyzed, and the damage evolution model was established using damage mechanics theory based on AE parameters.

\subsubsection{Load/Unload Response Ratio Theory}

Load/unload Response Ratio (LURR) theory can be defined as the specific value between the loading response and unloading response. LURR can be used to quantify the status and degree of failure of a nonlinear system. Therefore, it can be used to predict rockburst. A schematic diagram is shown in Fig. 3.2, where the horizontal axis represents the responsive displacement $(S)$ and the vertical axis represents the load $(F)$.

Two parameters are defined to quantitatively distinguish the difference between the load/unload responses:

The response rate $X$ is defined as 
Fig. 3.2 Uniaxial load/ unload schematic diagram

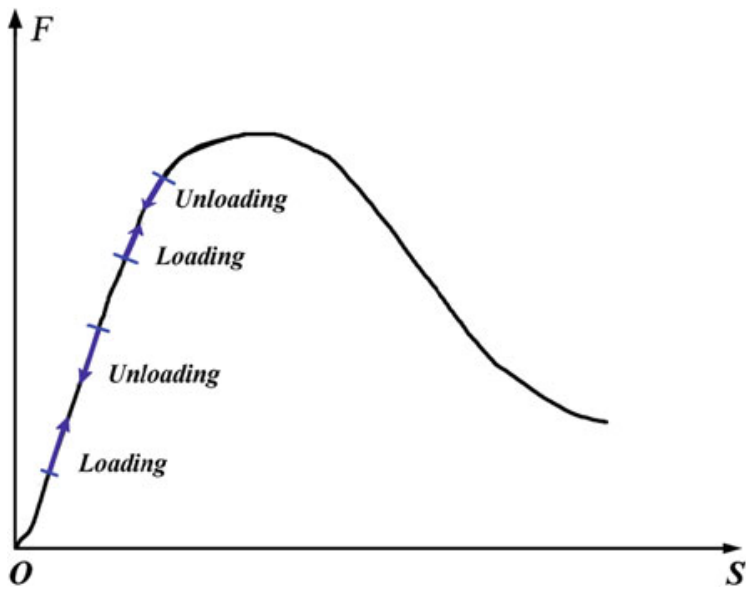

$$
X=\lim _{\Delta F \rightarrow 0}(\Delta S / \Delta F)
$$

where $\Delta F$ and $\Delta S$ are the increments of load $F$ and the responsive increments of displacement $S$, respectively.

LURR $Y$ is defined as

$$
Y=X_{+} / X_{-}
$$

where $X_{+}$and $X_{-}$are the response rates for the load/unload conditions, respectively. Then,

$$
\Delta F / \Delta S=k \cdot \mathrm{d} \sigma / \mathrm{d} \varepsilon=k \cdot E_{d}
$$

where $E_{d}$ is deformation modulus in $\mathrm{MPa}$ and $k$ is a constant, which is defined as

$$
k=A / l
$$

where $A$ is the cross-sectional area of cylindrical specimen $\left(\mathrm{m}^{2}\right)$ and $l$ is the length of rock specimen $(m)$. Therefore, the response rate $X$ is inversely proportional to the deformation modulus.

As shown in Fig. 3.1 and Eqs. (3.1)-(3.3), during the elastic deformation stage, the loading path is reversible, meaning that the load/unload moduli are equal. Therefore, during this stage, the loading response rate of rock specimens is equal to the unloading rate, or $X_{+}=X_{-}$. Based on Eq. (3.2), the $Y$ value (LURR) is equal to 1 , meaning that the deformation of rock specimens can be restored. During the plastic deformation stage, the loading response rate of rock specimens is greater than the unloading rate $\left(X_{+}>X_{-}\right)$. The $Y$ value (LURR) is greater than 1 , meaning that the physico-mechanical properties of rock specimens are reduced, such as 
deformation modulus, void ratio and compressive strength. As a result, LURR theory can be used to predict the degree of failure in the rock specimens.

Based on AE energy and $Y$ value (LURR) theory, AE energy is proposed as a response rate $(X)$ in Eq. (3.1). Then, the $Y$ value of load/unload energy response ratio (LUERR) can be defined as

$$
Y=\left[\sum_{i=1}^{N+} E_{i}^{m}\right]_{+} /\left[\sum_{i=1}^{N-} E_{i}^{m}\right]_{-}
$$

where $E$ is $\mathrm{AE}$ energy released in these experiments from the rock specimens, the sign ' + ' refers to loading, ' - ' refers to unloading, and $m=0,1 / 3,1 / 2,2 / 3$ or 1 . When $m=1, E^{m}$ is $\mathrm{AE}$ energy. When $\mathrm{m}=1 / 2, E^{m}$ is the Benioff strain, which is the square root of energy release for a sequential rockburst. When $m=1 / 3$ or $2 / 3$, $E^{m}$ is the $m$ root of energy release for a sequential coal burst. When $m=0, Y$ is equal to $\mathrm{N}_{+} / \mathrm{N}_{-}$, where $\mathrm{N}_{+}$and $\mathrm{N}_{-}$are the AE energies that occurred during the load/ unload operations, respectively. The values of $m=1 / 2$ and $m=1$ are adopted, meaning that $Y$ can be defined as

$$
\left\{\begin{array}{c}
Y=\left[\sum_{i=1}^{N+} E_{i}^{\frac{1}{2}}\right]_{+} /\left[\sum_{i=1}^{N-} E_{i}^{\frac{1}{2}}\right]_{-} \\
Y=\left[\sum_{i=1}^{N+} E_{i}\right]_{+} /\left[\sum_{i=1}^{N-} E_{i}\right]_{-}
\end{array}\right.
$$

\subsubsection{Entropy Theory}

Entropy is a thermodynamic concept and can be interpreted as a measure of disorder: the more disorganized a system, the higher its entropy. Entropy is a quantitative measure of uncertainty. Shannon, the founder of information theory, first systematically put forward a concept of measurement of information (Shannon 1948). Using the methods of probability and statistics, he took entropy as a measure of uncertainty or information of a random event, and solved the problem of quantitative measurement of information. For a probability test with $N$ results, set the results with each discrete probability $\lambda$. Meanwhile, if

$$
\begin{gathered}
0 \leq \lambda_{i} \leq 1 \quad(i=1,2, \ldots, n) \\
\sum_{i=1}^{n} \lambda_{i}=1
\end{gathered}
$$


Then

$$
H(X)=H\left(\lambda_{1}, \lambda_{2}, \ldots, \lambda_{n}\right)=-k \sum_{i=1}^{n} \lambda_{i} \log \lambda_{i}
$$

where $\mathrm{k}$ is a constant and $\mathrm{k} \geq 0 ; \mathrm{H}$ is the information entropy, which is also called probability entropy or Shannon entropy.

Rockmass deformation and failure is a nonlinear dynamic process of going from a disordered chaos into an ordered status. Thus, the concept of information entropy and the relevant principle can be used in rock failure. For all dominant frequency values, the entropy values were calculated using Matlab software. The supposed dominant frequency is $x(\mathrm{n})$, and $\mathrm{n}=1-\mathrm{N}$, is the quantity of AE signal waveforms. The detailed process is as follows,

(1) Find and group the distribution range of $x(\mathrm{n})$;

(2) Obtain the counts of dominant frequency data in each group at unit time interval, and solve the corresponding probability;

(3) Solve the entropy values using the information entropy formula.

\subsubsection{Fuzzy Matter Element Theory}

In order to analyze rockburst tendency more reasonably, the fuzzy matter element (FME) theory is applied to the evaluation of rockburst proneness. Cai (1994) devised the Matter Element Analysis (MEA) method with mathematics and experimental disciplines. In MEA, the three elements are events, features and values, which are used to describe these factors as the order basic element, and it is called matter-element. MEA is the study of matter-element variation and the system structure transforming, and the incompatibility problems are resolved if the magnitude of matter element are ambiguity. If the quantity of matter element is fuzzy, it constitutes a fuzzy incompatibility problem. FME was used to analyze and combine the fuzziness of corresponding quantity of things and the incompatibility between many factors, so as to obtain a new method for solving this kind of fuzzy incompatible problems. In recent years, this practical theory was being applied in the field of engineering technology, and many encouraging results were obtained.

There are many theories about rockburst tendency so far, such as stress method, rock integrity coefficient method, strength criterion etc. In these methods and the judgment index, if only the individual factors were considered, results may be one-sidedness and limitations. However, taking more factors into the complicated problem in rock engineering, many factors usually only have relative accuracy, and their relation with rockburst cannot be evaluated by "right" and "wrong". Therefore, it is necessary to make full use of FME to consider the more influencing 
factors of rockburst comprehensively and to make a comprehensive judgment of its proneness.

\subsubsection{Bayesian Theory and Network Model}

A Bayesian identifiable model, with a statistical analysis heritage, is used to distinguish among different types of samples. The primary procedure is based on an artificial familiarity with known samples and possible attendant consequences. Firstly, the empirical probability and covariance of each classification is analyzed and calculated. Then, a discriminant function is formulated to grade samples. Finally, a posterior probability is calculated to verify the original evaluation. New samples were easily classified after being input into model (Ren and Yu 1999; Gao 1999).

Bayesian statistics is the basis of Bayesian discriminant analysis. The basic idea of Bayesian statistics is as follow: Firstly, we must have a certain understanding of research object. The prior probability distribution is used to describe this kind of knowledge, and the posterior probability distribution is obtained based on the prior knowledge of extracted samples, then can do a variety of statistics based on the posterior probability distribution. Finally, the Bayesian statistics can be used for discriminant analysis, and Bayesian discriminant analysis can be carried out as a basic method of statistical model in decision making, in all cases is not completely known for some unknown status with subjective probability estimation, the probability is modified by the Bayesians formula, finally using the expected value and correction probability to make decision. Bayesians theory has the uncertain knowledge expression ability and flexible reasoning mechanism, Bayesians' theory of rockburst tendency prediction model based on combining rockburst cases studied systematically in order to seek for effective prediction of rockburst. Combined with Bayesian distance discriminant analysis, a multi-parameter and multi-dimension rockburst predicting models can be established to overcome the limitations of single factor and individual difference.

\section{References}

Brauner G (1994) Rockbursts in coal mines and their prevention. CRC press, AA Balkema

Cai W (1994) Matter element model and its application. Scientific and Technical Documents Publishing House, Beijing (in Chinese)

Chen BR, Feng XT, Li QP et al (2015) Rock burst intensity classification based on the radiated energy with damage intensity at Jinping II hydropower station. China Rock Mech Rock Eng 48 (1):289-303 (in Chinese)

Gao HX (1999) Multivariate statistical analysis application. Beijing University Press, Beijing 
He MC, Miao JL, Feng JL (2010) Rock burst process of limestone and its acoustic emission characteristics under true-triaxial unloading conditions. Int J Rock Mech Min Sci 47(2):286298

Hill FG, Cook NGW, Hoek E et al (1966) Rock mechanics applied to study of rockbursts. J S Afr Inst Min Metall 35(1):435-528

Hult J (1975) Damage-induced tensile instability. Structural Mechanics in Reactor Technology Press, London

Kachanov LM (1958) Time of the rupture process under creep conditions. Isv. Akad Nauk SSR Otd Tekh Nauk 8:26-31

Leckie FA, Hayhurst DR (1974) Creep rupture of structures. In: Proceedings of the Royal Society of London A: mathematical, physical and engineering sciences, vol 340, no 1622, The Royal Society, London, pp 323-347

Leighton F (1982) A case history of a major rock burst. U.S Department of the Interior, Bureau of Mines

Lemaitre J Chaboche JL (1974) A non-linear method of creep-fatigue damage acummulation and interaction. In: Proceedings IU TAM Symposium Viscoel, Gothenburg

Lindin GL, Lobanova TV (2013) Energy sources of rockbursts. J Min Sci 49(1):36-43

Ma TH, Tang CA, Tang XL et al (2016) Mechanism of rock burst forecasting based on micro-seismic monitoring technology. Chin J Rock Mechan Eng 35(3):470-483 (in Chinese)

Pan YS, Xiao YH, Li ZH et al (2014) Study of tunnel support theory of rockburst in coal mine and its application. J China Coal Soc 39(02):222-228

Rabotnov YN (1969) Creep rupture. Springer, Berlin, Heidelberg, pp 342-349

Ren XS, Yu XL (1999) Multivariate statistical analysis. China Statistics Press, Beijing

Sato K, Isobe T, Mori N et al (1986) Microseismic activity associated with hydraulic mining. Int J Rock Mech Mining Sci Geomech Abstracts 23(1):85-94

Shannon CE (1948) A mathematical theory of communication. Bell Syst Tech J 27(3):379-423

Shen W (2006) Dissipative structure, self-organization catastrophe theory and earth science. Harbin Institute of Technology Press, Harbin

Wang CL (2014) Identification of early-warning key point for rockmass instability using acoustic emission/microseismic activity monitoring. Int J Rock Mech Min Sci 71:171-175

Wang CL, Wu AX, Lu H et al (2015) Predicting rockburst tendency based on fuzzy matterelement model. Int J Rock Mech Min Sci 75:224-232

Zhao F (2015) Experimental analysis of important factors affecting rock burst. Master's thesis, China University of Mining and Technology (Beijing), Beijing, China (in Chinese)

Open Access This chapter is licensed under the terms of the Creative Commons Attribution-NonCommercial 4.0 International License (http://creativecommons.org/licenses/by-nc/ $4.0 /$ ), which permits any noncommercial use, sharing, adaptation, distribution and reproduction in any medium or format, as long as you give appropriate credit to the original author(s) and the source, provide a link to the Creative Commons license and indicate if changes were made.

The images or other third party material in this book are included in the book's Creative Commons license, unless indicated otherwise in a credit line to the material. If material is not included in the book's Creative Commons license and your intended use is not permitted by statutory regulation or exceeds the permitted use, you will need to obtain permission directly from the copyright holder.

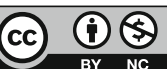




\section{Chapter 4 \\ Three-Dimensional Reconstruction Model and Numerical Simulation of Rock \\ Fissures}

\subsection{Introduction}

The problem of rockmass sudden destruction is closely related to the existence and evolution of fissures. The development and evolution of rock fissures under loading pass three stages: the stage of secondary crack initiation, the stage of formation of macro-fracture zone and the stage of localized deformation. In this chapter, three-dimensional reconstruction model of rock fracture was carried out in order to further reveal the damage mechanism of rockmass and the evolution pattern of fracture cracks. In order to further reveal the damage mechanism of rockmass instability, predicting rockburst during the process of damage evolution and rockmass instability was organically combinated.

With the development and improvement of computer technology, the digital image processing technology has gradually developed into an emerging discipline. It mainly converts the changing process of object into a digital image stored in an electronic computer, and application of computer to deal with image information obtained and analysis to get the required results. Although many scholars applied digital image processing technology to quantitatively describe and effectively reveal the evolution and damage of cracks. A lot of work was analyzed using CT image and three-dimensional reconstruction model of fissures (Kawakata et al. 1999, 2000). Moreover, using the X-ray, CT scanning experiment was used to simulate the joint distribution and trend of rockmass (Ueta et al. 2000). Three-dimensional reconstruction model and numerical simulation of CT images were established on the basis of digital image. It is necessary to use numerical simulation for more accurate characterization and description of rock and reflecting the actual force of rockmass. Therefore, based on the digital image processing technique, there is a new approach to study the mechanical properties of rocks by analyzing the CT scan image and the three-dimensional reconstruction model.

Kawakata et al. (1999) conducted three-dimensional reconstruction model of two-dimensional cracks images using the CT scan data of rock samples, and made 
analysis of shape and spatial distribution of cracks. Ueta et al. (2000) carried out three-dimensional reconstruction model of indoor sandstone during shear; the shear zone of inner space was reproduced, and analyzed the intersected structure of outer sedimentary layer. Ruiz de Argandona et al. (1999) studied the pore structure of rocks using CT scanning technology, and reconstructed the three-dimensional pore structure of rock, the inhomogeneity of pore distribution in rock was revealed. Using CT image information, the extension evolution of rock weakening and pore was dynamically tracked and described. Wang et al. (2003) studied the damage of mechanical properties of concrete materials using of CT scanning technology, the concrete two-dimensional full-scan images were obtained, three-dimensional reconstruction model was verified the relationship between damage parameters and mechanical modeling. Viggiani et al. (2004) assessed the evolution and development of shear bands by analyzing the spatial resolution X-rays under a fine-grained hard soil partial load.

Bornert et al. (2010) studied the strain localization characteristics of clay rocks under triaxial compression using the X-ray micro CT. Feng et al. (2004) investigated the mechanism of sandstone damage evolution by real-time $\mathrm{CT}$, and proposed a damage model based on chemical corrosion and CT value. Louis et al. (2006) solved the positioning features of 3D geometric shapes and develop them into the braces using the high resolution X-ray CT imaging technique, and obtained the associated with the maximum compressive stress. Nasseri et al. (2011) obtained the reasonable fracture toughness anisotropy of Bale granite, and reflects the difference of damage structure using a three-dimensional object counter plug-in technique method. Chen et al. (2007) proposed a three-dimensional numerical simulation methods based on digital images of rock failure.

Three-dimensional reconstruction model technique can be used to observe the changes of geotechnical structure under multi-dimension and without disturbance, and observe the microstructural changes of samples, the movement of meso-particles, the development of fissures, and the local density. The three-dimensional reconstruction model technique realizes the visualization of cracks distribution at different stages of loaded rock samples. It can not only visually observe the evolution of rock failure, but also describe the spatial distribution of medium of rock material accurately. Based on the image data of rock using CT scan, this chapter used the digital image processing technology to extract the useful information from the CT images and reconstruct the three-dimensional model based on MATLAB software. 


\subsection{CT Scanning Experiment Under Uniaxial Cyclic Load/Unload}

In load/unload test, the rock samples generated many microcracks under the external load, the microcrack initializes, expands and penetrates, eventually leading to macroscopic destruction. In order to further reveal the predicting of rockburst, it is necessary to study the evolution pattern of internal microstructure of rock samples. CT scanning can be used to obtain the internal structural changes of rock samples. CT images, obtained by CT scanning, can analyze the initiation, expansion, penetrating and final evolution process of microcrack of rock samples.

CT scanning techniques can eliminate interference from other parts of object in the target area, and make objects appear in two-dimensional images in isolation, which can help improve the quality of image. The material composition of object and the damage location are also more clearly and accurately. CT scanning technology is described as follows: Detection receivers and X-ray sources are fixed on the same rack. Then the rack rotates a fixed angle, which indicates the end of a scan, and the detected objection will be in synchronous rotation with the rack. The rack will rotate the same angle at the end of each scan to complete the next scan. The multi-group scanning data can be collected by repeated rotating scanning process. Complete a scan, the rack will rotate once, and 256 data will be collected, if the rack rotates $180^{\circ}, 46,080$ data will be collected. CT images of each scanning level of detected object can be gotten by processing these collecting dates.

In this experiment, based on the result of sandstone samples failure under unaxial loading, the unaxial compressive strength (UCS) of roof sandstone sample is $93.48 \mathrm{MPa}$. Therefore, its UCS under cyclic load/unload can be estimated as $90 \mathrm{MPa}$; the UCS of floor sandstone sample is $58.18 \mathrm{MPa}$. Therefore, its UCS under cyclic load/unload can be estimated as $60 \mathrm{MPa}$; as shown in Fig. 2.7, CT scanning test of sandstone is divided into two phases under unaxial cyclic load/ unload. Upper and lower stress of axial loading at the first stage of cyclic load/ unload is respectively 10 and $30 \%$ of UCS; Upper and lower stress of axial loading at the second stage of cyclic load/unload is respectively 30 and $70 \%$ of UCS, the sample will then be loaded and destroyed. Axial stress is used to control loading. The loading method is cyclic load/unload by constant amplitude; the loading rate of roof sandstone is $500 \mathrm{~N} / \mathrm{s}$, floor sandstone is $300 \mathrm{~N} / \mathrm{s}$. The triangle waveform is selected for loading, in order to ensure that the time of load/unload process is equal, load/unload speed should be consistent (Fig. 4.1).

The specific scanning process of this experiment is as follows:

(1) An initial scan of roof and floor sandstone sample load/unload was carried out to obtain the mesoscopic structure image of rock sample before the cyclic load/unload. 


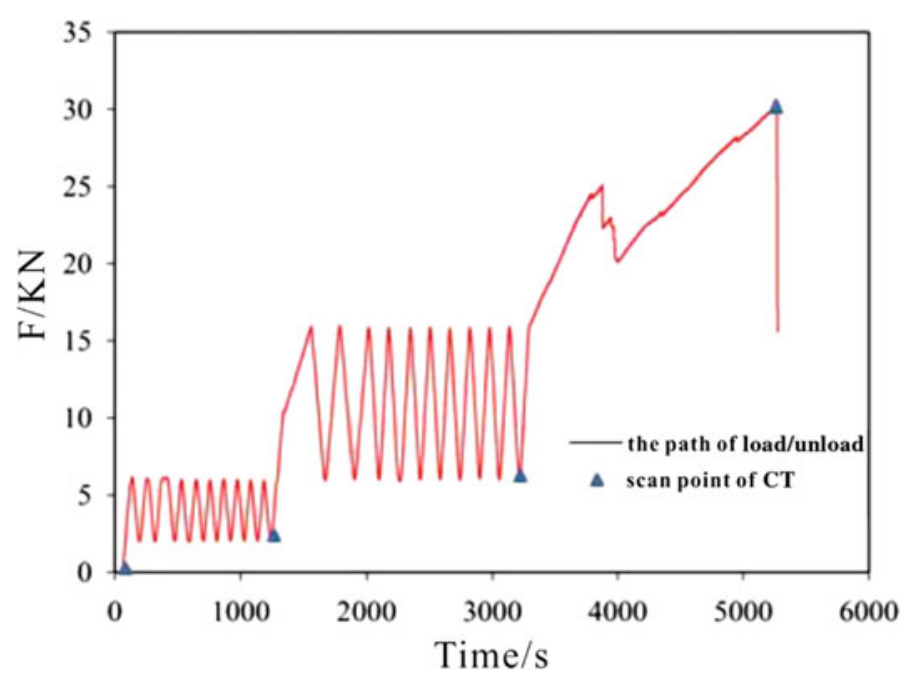

Fig. 4.1 Scan location and path of load/unload of rock sample

Table 4.1 The parameters of

\begin{tabular}{l|l|l|l}
\hline The sample size & $\begin{array}{l}\text { Number of } \\
\text { pieces }\end{array}$ & $\begin{array}{l}\mathrm{U} \\
(\mathrm{kV})\end{array}$ & $\begin{array}{l}\mathrm{I} \\
(\mu \mathrm{A})\end{array}$ \\
\hline$\Phi 50 \times 100$ & 1000 & 145 & 300 \\
\cline { 3 - 4 } & & 110 & 200 \\
\hline
\end{tabular}

(2) Stop loading after 10 cycles at the first stage under cyclic load/unload, completing the second scan of rock sample. The internal microstructure images of roof and floor sandstone load/unload were obtained;

(3) Stop loading after 10 cycles at the second stage under cyclic load/unload, the third scan of rock sample was completed. The internal microstructure images of roof and floor sandstone load/unload were obtained;

(4) Complete the fourth scan of rock sample at the stage of rock failure; the internal microstructure images of roof and floor sandstone after the stage of rock failure were obtained. The parameters of CT scanning were set as Table 4.1.

\subsection{Cracks Extraction and Calculation}

\subsubsection{Processing the CT Images}

\subsubsection{Characteristics of CT Images Information}

CT image of rock samples are qualitative and quantitative information extraction. It is important to consider the possibility of the repeated scanning in the test design. 
The selection of scanning phase should be able to fully reflect the evolution of rock failure, the change of images are be described qualitatively or quantitatively, the occurrence of abnormal conditions are be observed. Repeated scanning experiments are carried out to ensure the accuracy of test results, and it is necessary to detailedly be described the regularity characteristics of rock failure, the error was eliminated in the process of preparing samples, the experimental results were be compared under different loading methods. In order to accurately and effectively analyze the internal structure changes of material presented in the images, it is necessary to fully know about the lithological characteristics of samples, the change of these loaded rock samples is be mastered because of the internal particle friction or the composition of internal matter, the results are due to the change of proportion of a substance or the change of substance density. Avoiding the influence of various objective factors on the images, the change of images can be represented by the abnormal area. In conclusion, it is very important to deeply understand the process of rock failure using the CT scan data.

\subsubsection{CT Image Processing Contents and Methods}

1. CT image processing contents

(1) Arithmetic of image

1) Plus. For the image that continues to be transformed in one direction, the algorithm can refine this change using the weighted average of image successive layers, which can improve the spatial resolution of image to some extent.

2) Subtract. It is a very effective method to detect the internal material migration of samples. Because the change of image data is much smaller than the data itself, this operation can greatly reduce the original artifact of CT images.

3) Multiply. Multiplying the image by a constant, a distribution function, or another image, it can be enhanced to correct or alter the original image to achieve a particular target.

4) Divide. It has the same effect as multiplication.

5) Combinatorial operations. The image is performed on a cumulative basis according to the expected target.

(2) Image enhancement

The meaning of enhanced processing is obtained using some specific information on the change of CT number or expression method of image, while it may change the quantitative significance of CT number. Common methods are as follow:

1) High pass filtering (differential). In the area, gradient technology can be used to focus the image on the image. Using exponential or other 
convolution filtering can improve the image characteristics of center or outer edge. After taking the Fourier transform in the frequency domain, the high-pass filtering is carried out, then these images of inverse Fourier transform is shown, and the spatial change of image is highlighted. Limited high-pass filtering can make the boundary clear, and the recognition of images can be helpful.

2) Low pass filtering (on average). The spatial and frequency domain is used to reduce the parasitic effects of detector space sampling and incomplete algorithm. Although some spatial resolution is overflowed, the image data are more reliable and the image is more gentle and coherent.

3) Histogram equalization. The entire image brightness everywhere maybe cause that the image can't be found at the same time, equalization processing can improve dark space value, reduce light area, it is easier to see these details of image; if the distribution of image values is measured, then these images histogram equalization is used to adjust the data, and the obtained images have better results.

4) Direction filtering. In order to highlight some features of orientations, they can be highlighted, and the less obvious structures are identified.

(3) Image segmentation

Image segmentation is to extract the need to study in the image of local area and according to the regional characteristics of entire process of image classification, through the region of interest in the segmented regions extracted, in order to achieve the goal of observation and analysis. According to the image processing technology, the following two principles are used to divide the research images:

1) The gray value of each pixel is not necessarily continuous in the image, so it can be divided.

2) Due to the similarity of gray values in the same region, the grayscale differences in different regions can be found to be divided.

The above two segmentation methods have certain limitations for different images. Achieving the satisfactory segmentation results, we need have a thorough understanding of image information. Reasonable segmentation methods are selected by its characteristics. Image segmentation can be used to extract the images of rock and damage zones (including holes, microcracks and microdamage that human eyes can hardly observe), and these characteristics that image segmentation is based on may be pixel gray, gray variance of small area, the gray scale distribution, grayscale texture, image point or the color of small area and so on.

(4) Measurement of image data

After mastering the method of image processing on software, a series of images changes of data information can be collected and calibrated in a 
specific way. These continuous collections of data in the same area can be used to analyze the changes in this region. Duo to the data fluctuation range of limited area is small; it is difficult to calibrate the region. In the large area, the area larger than $10 \mathrm{~mm}^{2}$ is normally selected. In order to avoid the influence of data error caused by image distortion, the study area must be tracked.

(5) Distribution of image data

Measuring the gray value of cracks in certain region at different loading stages, the internal structure of rock samples can be studied from the perspective of mesoscopic, which can help to understand the rock damage mechanism more deeply.

2. CT image processing

(1) Image preprocessing. In the process of collecting data of CT images, the influence of surrounding environment and scanning equipment running parts produced by quantization noise affect data collection, the image quality was reduced, the image blur were made, the essential features of images were changed and eliminated, and it was not conducive to the recognition of CT image and analysis. Therefore, it is very important to carry out the initial treatment of CT images without destroying the image information and characteristics. There are many methods for the preprocessing of CT scan images, including brightness adjustment and contrast adjustment. The CT images were imported into MATLAB software; Firstly, the grayscale images could change by grayscale transformation, the scale function provided by the MATLAB toolbox, which would enhance the image contrast. Secondly, the combination of high and low data transformation made the foreground and background gray of image further stretched, highlighting relevant targets and details to enhance the image's function. In the end, the image data was reversed and the image was further enhanced.

(2) Image segmentation. Images were segmented for realizing effectively in the research area, mesoscopic cracks of rock scanned images existed, reducing the influence on image information of all kinds of objective conditions caused by accidental error in the process of CT scanning. Firstly, using the threshold transform to convert grayscale image to binary image, which referred to the image with only black (threshold value 0) and white (threshold value 255) in the image. Secondly, using the morphological operations of image expansion and open the binary region respectively for binary image "extended", "bold" and delete in the binary image area, this was less than a certain value of object. Getting the clear cracks, the image of each processing stage is shown in Fig. 4.2. 


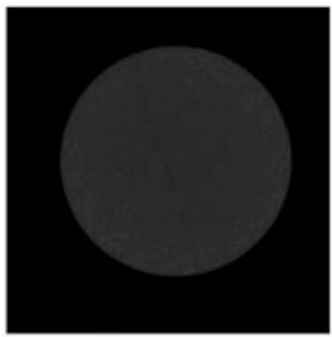

(a)The original image

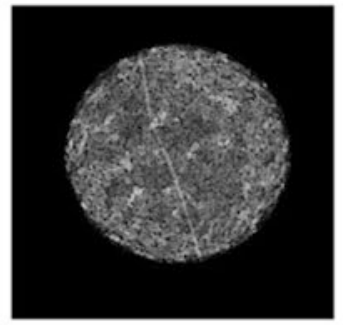

(d)Bottom-Hat transform

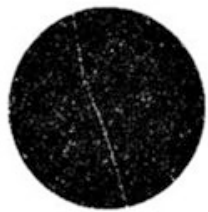

(g)Binarization

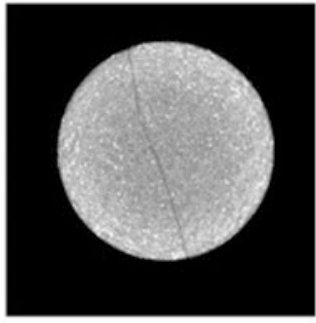

(b)Linear transformation

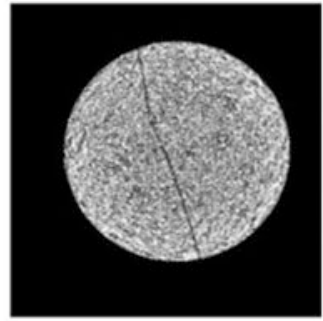

(e) Image subtract

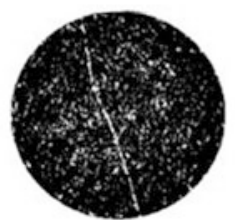

(h)Image expansion

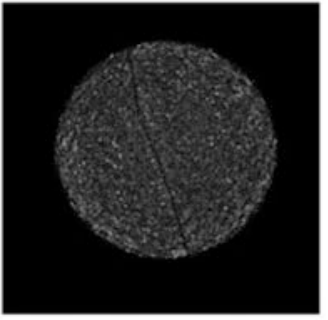

(c)Top-hat transform

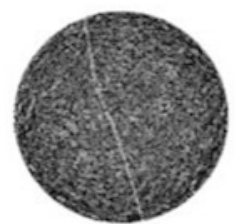

(f)Negate operation

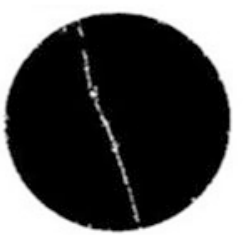

(i) Remove small area objects

Fig. 4.2 Cracks images at each processing stage

\subsubsection{Calculating the Length and Area of Cracks}

\subsubsection{Calculating the Length of Cracks}

The length of cracks were straightened, the number of pixels, occupied by the cracks in the image area, could be obtained by statistical calculation, and then the lengths of cracks could be calculated. In this chapter, the bwperim function in MATLAB software was used to calculate the located cracks length in the region. The edge pixels of cracks could be extracted in the binarized images. The edge pixels of cracks must meet the following conditions: Value is 1 (the white area is the crack area whose pixel value is " 1 "), and at least one value in the adjacent area is 0 , and the bwperim function can display the edge contour of cracks in the images.

The calculation results of cracks lengths at each layer of roof sandstone at different stress conditions are shown in Tables 4.2, 4.3 and Figs. 4.3, 4.4. We can 
Table 4.2 Cracks lengths at each layer of roof sandstone at different stress conditions

\begin{tabular}{l|l|l|l|l}
\hline $\begin{array}{l}\text { Roof } \\
\text { sandstone }\end{array}$ & $\begin{array}{l}\text { Before } \\
\text { loading } \\
(0 \mathrm{MPa})\end{array}$ & $\begin{array}{l}\text { After the first } \\
\text { phase }(9 \mathrm{MPa})\end{array}$ & $\begin{array}{l}\text { After the second } \\
\text { phase }(27 \mathrm{MPa})\end{array}$ & $\begin{array}{l}\text { After failure } \\
(89.1 \mathrm{MPa})\end{array}$ \\
\hline 350 layer & $4.2956 \times 10^{3}$ & $4.5681 \times 10^{3}$ & $4.5968 \times 10^{3}$ & $6.3309 \times 10^{3}$ \\
\hline 450 layer & $4.3096 \times 10^{3}$ & $4.6249 \times 10^{3}$ & $4.8052 \times 10^{3}$ & $6.0482 \times 10^{3}$ \\
\hline 550 layer & $4.1452 \times 10^{3}$ & $4.3248 \times 10^{3}$ & $4.5743 \times 10^{3}$ & $4.9707 \times 10^{3}$ \\
\hline 650 layer & $4.2589 \times 10^{3}$ & $4.3650 \times 10^{3}$ & $4.5944 \times 10^{3}$ & $6.2999 \times 10^{3}$ \\
\hline
\end{tabular}

Table 4.3 Cracks lengths at each layer of bottom sandstone at different stress conditions

\begin{tabular}{l|l|l|l|l}
\hline $\begin{array}{l}\text { Bottom } \\
\text { sandstone }\end{array}$ & $\begin{array}{l}\text { Before } \\
\text { loading } \\
(0 \mathrm{MPa})\end{array}$ & $\begin{array}{l}\text { After the first } \\
\text { phase }(6 \mathrm{MPa})\end{array}$ & $\begin{array}{l}\text { After the second } \\
\text { phase }(18 \mathrm{MPa})\end{array}$ & $\begin{array}{l}\text { After failure } \\
(37.5 \mathrm{MPa})\end{array}$ \\
\hline 350 layer & $4.4808 \times 10^{3}$ & $4.5974 \times 10^{3}$ & $5.0556 \times 10^{3}$ & $8.0700 \times 10^{3}$ \\
\hline 450 layer & $5.0623 \times 10^{3}$ & $5.1282 \times 10^{3}$ & $5.9059 \times 10^{3}$ & $8.4840 \times 10^{3}$ \\
\hline 550 layer & $4.8902 \times 10^{3}$ & $5.0337 \times 10^{3}$ & $5.5458 \times 10^{3}$ & $8.9168 \times 10^{3}$ \\
\hline 650 layer & $4.5148 \times 10^{3}$ & $4.5900 \times 10^{3}$ & $5.0070 \times 10^{3}$ & $8.1470 \times 10^{3}$ \\
\hline
\end{tabular}

found that the cracks lengths of bottom and floor sandstone increased indifferent layers as the cyclic load/unload. From the beginning of unloading phase to the end of the first phase under cyclic load/unload, the cracks growth was small and the surfaces of sandstone were not significantly damaged. From the end of the first stage load/unload to the second stage of cyclic load/unload, the cracks growth were more obvious and the cracking rate of sandstone accelerates. From the second stage of cyclic load/unload to the final failure of sample, the cracks length increased rapidly, and the fracture of sandstone intensified.

\subsubsection{Calculating the Area of Cracks}

The bwarea function of MATLAB software was used to calculate the area with pixel value of 1 in binary image. It could be found that the pixel values in the cracks binary image were 1 pixel area. Different pixel models could use the bwarea function to give different values, and the area of interest target could be obtained by weighting the sum. In order to compensate for the deformation of continuous image with discrete pixel, it must be weighted. For example, a horizontal and vertical image consisting of 50 pixels had a smaller area than the 50-pixel diagonal images. 50pixel can represent the area of image under both horizontal and vertical conditions. However, the area of diagonal image was 62.5 because of weighted operation. The total area of the bwarea function was obtained by summing up all the individual pixel area in the image and the area of single pixel was obtained according to its $2 \times 2$ zones. There were six models for calculating individual pixels. 


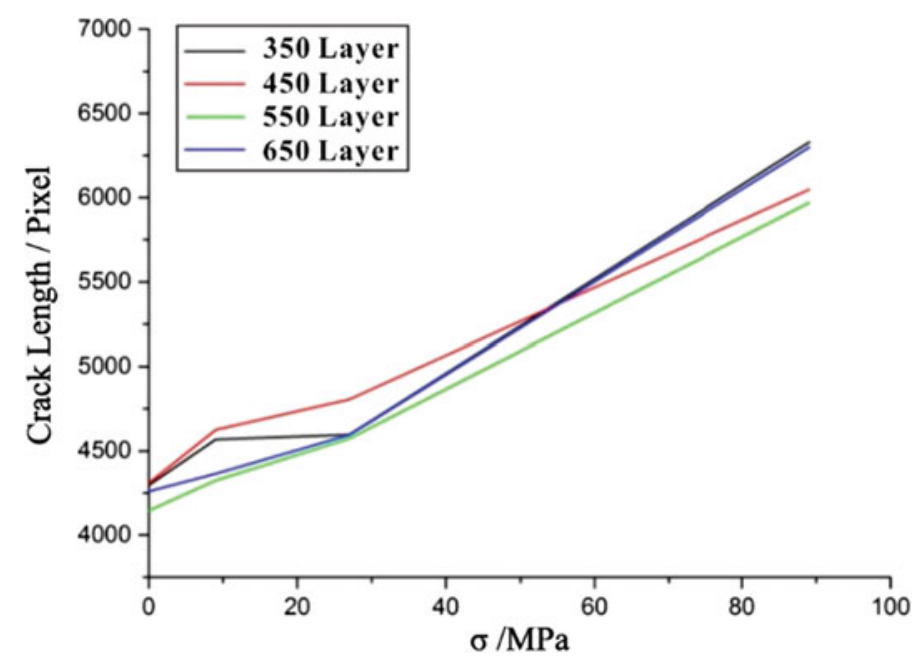

Fig. 4.3 The tendency of cracks length at each layer of roof sandstone at different stress conditions

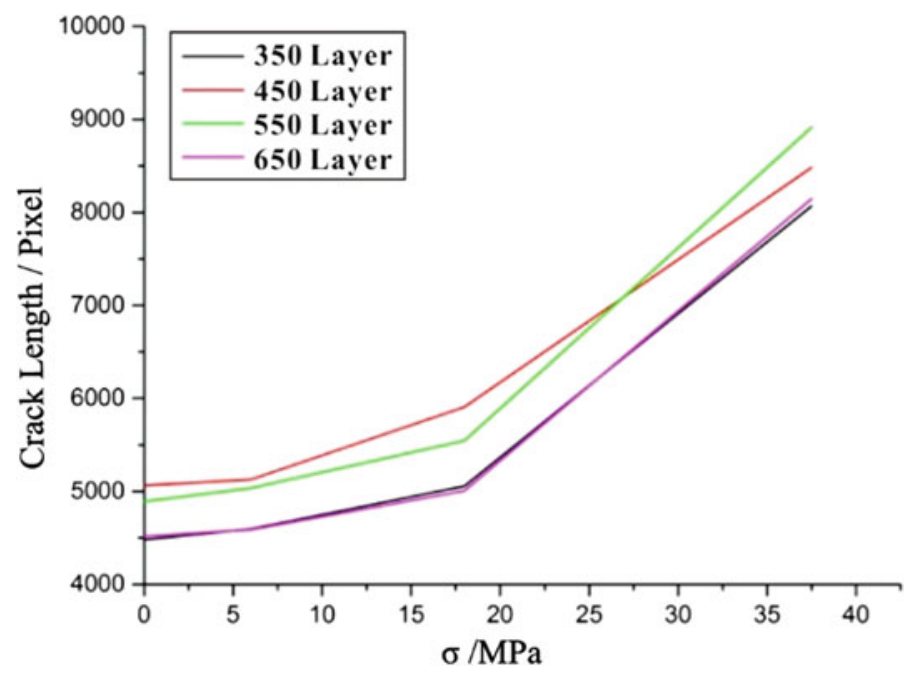

Fig. 4.4 The tendency of cracks lengths at each layer of bottom sandstone at different stress conditions

In the adjacent region, there is no pixel value of " 1 ", and the individual pixel area is 0 ; one pixel has a pixel value of " 1 " and the single pixel area is $1 / 4$; there are two adjacent pixels with the pixel value of "1" and the single pixel area is $1 / 2$; there are two pixels with the value of " 1 ", and the single pixel area is $3 / 4$; there are three pixels with a point of " 1 ", and the area of a single pixel is $7 / 8$; there are four pixels with a point of "1" and the area of a single pixel is 1 . 
Each pixel in a binary image can be viewed as a part of a neighboring region of 4 different $2 \times 2$. Therefore, there is no contiguous, diagonally connected pixel value of " 1 ", and the total area is 1 . The calculation method of BW area of binary image is as follows:

$\begin{array}{llllllll}0 & 0 & 0 & 0 & 0 & 0 & 0 & 0 \\ 0 & 0 & 0 & 0 & 0 & 0 & 0 & 0 \\ 0 & 0 & 1 & 1 & 0 & 0 & 0 & 0 \\ 0 & 0 & 1 & 1 & 0 & 0 & 0 & 0 \\ 0 & 0 & 0 & 0 & 0 & 0 & 0 & 0 \\ 0 & 0 & 0 & 0 & 0 & 1 & 0 & 0 \\ 0 & 0 & 0 & 0 & 0 & 0 & 0 & 0 \\ 0 & 0 & 0 & 0 & 0 & 0 & 0 & 0\end{array}$

The result of calculation is 5 .

The calculation results of cracks area at each layer of roof sandstone at different stress conditions were shown in Tables 4.4, 4.5 and Figs. 4.5, 4.6. We could found that the cracks area of bottom and floor sandstone was increased at different layers as the cyclic load/unload. The evolution was similar as shown in Tables 4.2, 4.3 and Figs. 4.3, 4.4.

Table 4.4 Cracks areas at each layer of roof sandstone at different stress conditions

\begin{tabular}{l|l|l|l|l}
\hline $\begin{array}{l}\text { Roof } \\
\text { sandstone }\end{array}$ & $\begin{array}{l}\text { Before } \\
\text { loading } \\
(0 \mathrm{MPa})\end{array}$ & $\begin{array}{l}\text { After the first } \\
\text { phase }(9 \mathrm{MPa})\end{array}$ & $\begin{array}{l}\text { After the second } \\
\text { phase }(27 \mathrm{MPa})\end{array}$ & $\begin{array}{l}\text { After failure } \\
(89.1 \mathrm{MPa})\end{array}$ \\
\hline 350 layer & $2.6581 \times 10^{5}$ & $2.6653 \times 10^{5}$ & $2.6754 \times 10^{5}$ & $2.7238 \times 10^{5}$ \\
\hline 450 layer & $2.6624 \times 10^{5}$ & $2.6632 \times 10^{5}$ & $2.6721 \times 10^{5}$ & $2.7008 \times 10^{5}$ \\
\hline 550 layer & $2.6533 \times 10^{5}$ & $2.6613 \times 10^{5}$ & $2.6658 \times 10^{5}$ & $2.7019 \times 10^{5}$ \\
\hline 650 layer & $2.6619 \times 10^{5}$ & $2.6693 \times 10^{5}$ & $2.6735 \times 10^{5}$ & $2.7142 \times 10^{5}$ \\
\hline
\end{tabular}

Table 4.5 Cracks areas at each layer of bottom sandstone at different stress conditions

\begin{tabular}{l|l|l|l|l}
\hline $\begin{array}{l}\text { Bottom } \\
\text { sandstone }\end{array}$ & $\begin{array}{l}\text { Before } \\
\text { loading } \\
(0 \mathrm{MPa})\end{array}$ & $\begin{array}{l}\text { After the first } \\
\text { phase }(6 \mathrm{MPa})\end{array}$ & $\begin{array}{l}\text { After the second } \\
\text { phase }(18 \mathrm{MPa})\end{array}$ & $\begin{array}{l}\text { After failure } \\
(37.5 \mathrm{MPa})\end{array}$ \\
\hline 350 layer & $1.3171 \times 10^{5}$ & $1.3185 \times 10^{5}$ & $1.3243 \times 10^{5}$ & $1.3683 \times 10^{5}$ \\
\hline 450 layer & $1.3253 \times 10^{5}$ & $1.3260 \times 10^{5}$ & $1.3383 \times 10^{5}$ & $1.3772 \times 10^{5}$ \\
\hline 550 layer & $1.3223 \times 10^{5}$ & $1.3232 \times 10^{5}$ & $1.3309 \times 10^{5}$ & $1.3626 \times 10^{5}$ \\
\hline 650 layer & $1.3179 \times 10^{5}$ & $1.3185 \times 10^{5}$ & $1.3235 \times 10^{5}$ & $1.3646 \times 10^{5}$ \\
\hline
\end{tabular}




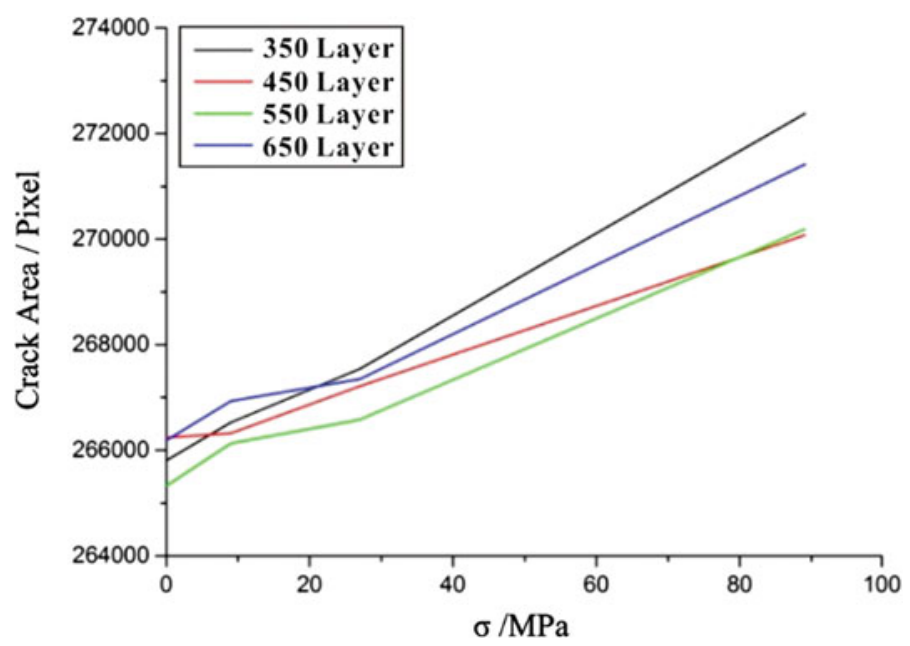

Fig. 4.5 The tendency of cracks area at each layer of roof sandstone at different stress conditions

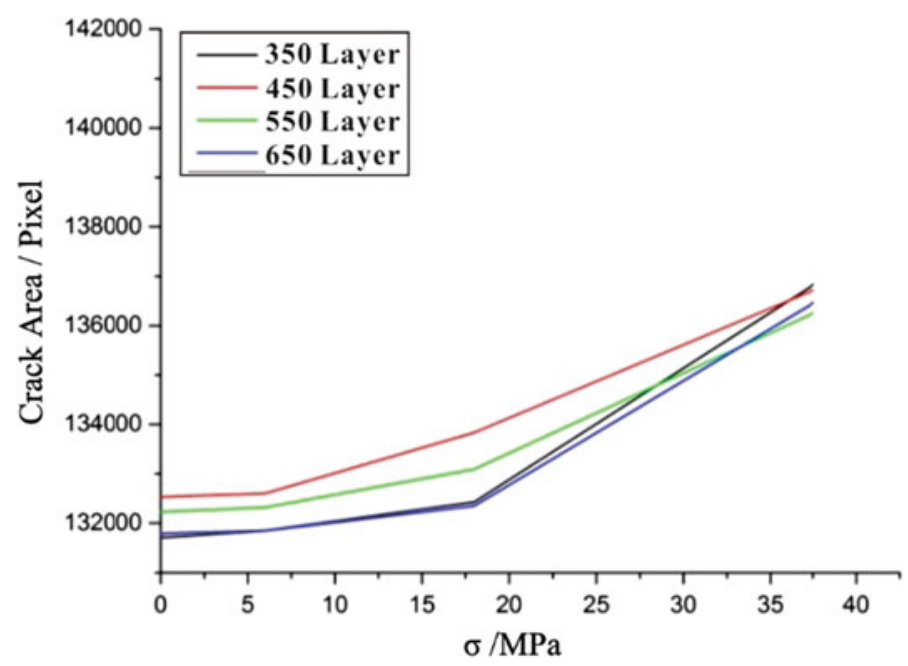

Fig. 4.6 The tendency of cracks area at each layer of bottom sandstone at different stress conditions

\subsection{Three-Dimensional Reconstruction Model and Numerical Simulation}

In order to obtain the influence of mesostructure evolution of rock failure on its macro-mechanical properties, sandstone specimens were scanned by CT. The MIMICS software was used to reconstruct the two-dimensional images of 
fissured rock at the stage of unloading, the microscopic characteristics of sandstone could be reproduced by the reconstructed numerical model. The mechanical response of sandstone specimens was simulated by FLAC software under uniaxial cyclic load/unload.

\subsubsection{Establishing a Three-Dimensional Finite Element Model}

\subsubsection{Establishing the Model of Fissured Rock}

CT scanning was the process of dividing a three-dimensional rock sample into two layers of two-dimensional sections, and then completing the top-down scanning of rock specimens. Using the two-dimensional information obtained from the CT scanning, the $3 \mathrm{D}$ reconstruction of scanned object can be achieved by reverse modeling. If the three-dimensional solid model of real cracks structure in the rock was completed, the cracks structure with uneven distribution in each layer of sandstone was extracted, and then the mesh was divided. The three-dimensional finite element calculation of rock sample could be obtained.

\subsubsection{Three-Dimensional Reconstruction Model of Failured Sandstone}

The concrete process of reconstructing fractured rock under unloaded condition is as follows:

(1) The MATLAB software was used to scan the bin format into TIF format image files, put it into the $3 \mathrm{D}$ reconstruction software, and set the relevant parameters of actual test. The interval scanning was the picture size spacing, and the pixel size was:

$$
\mathrm{a}=\frac{b}{1024}
$$

where the size of scanned image was $1024 \times 1024$ px, and the size of pixel was shown with A. The length of square image was displayed with B. Finally, according to the angle of observation, the direction of model was set.

(2) The software generated the corresponding threshold according to the gray distribution in the image, and the segmentation of region was completed according to the threshold. 
(3) The 3D model of rock sample was generated according to $3 \mathrm{D}$ reconstruction function in software, and the 3D model was not a body model for calculation.

(4) By the system with 3-matic, smoothing the model, the required 3D model could be obtained.

The sandstone images, which were extracted and processed, were analyzed by the $3 \mathrm{D}$ reconstruction software. In order to obtain the visual cracks distribution, three-dimensional model of rock samples needed to be set through a series of parameters. Different materials in the reconstruction of specimen could be distinguished by choosing different thresholds in the CT image, which was convenient for the division of cracks and the rock matrix. The distribution of cracks structure could be reflected in the three-dimensional model of fractured sandstone. The three-dimensional models of rock fracture were shown in Figs. 4.7 and 4.8.

Fig. 4.7 Three-dimensional reconstruction model of fractured rockmass

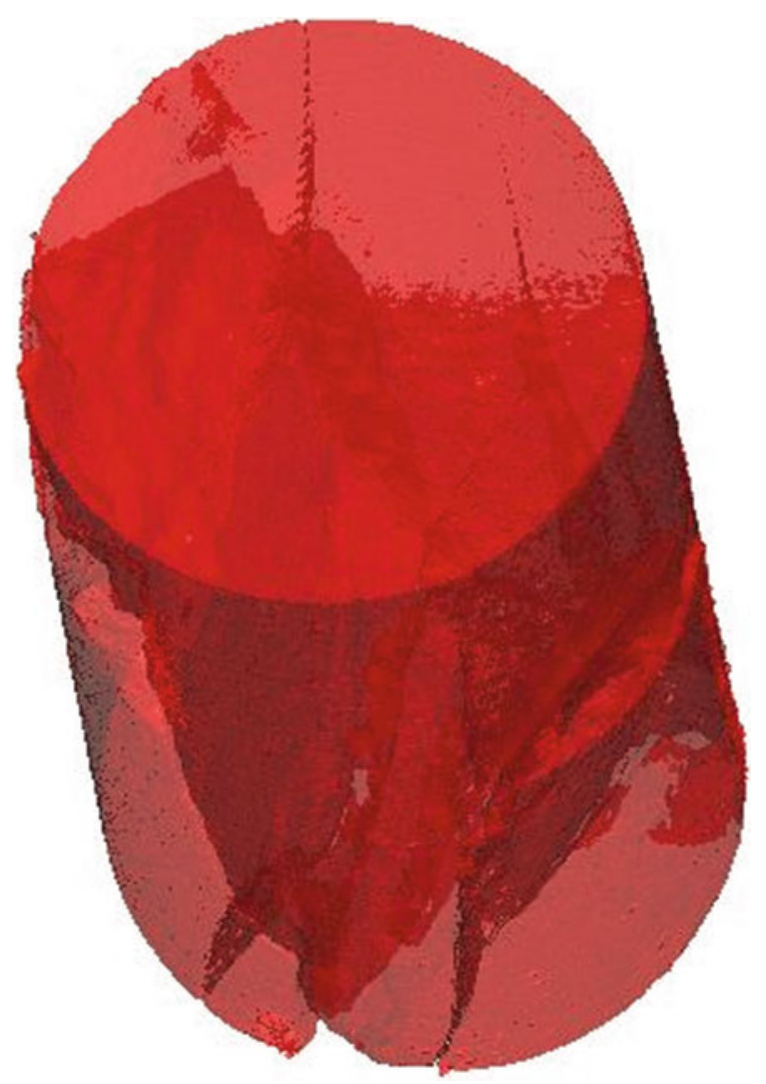


Fig. 4.8 Sectional view of $\mathrm{X}-\mathrm{Y}$ cross section of fractured rockmass

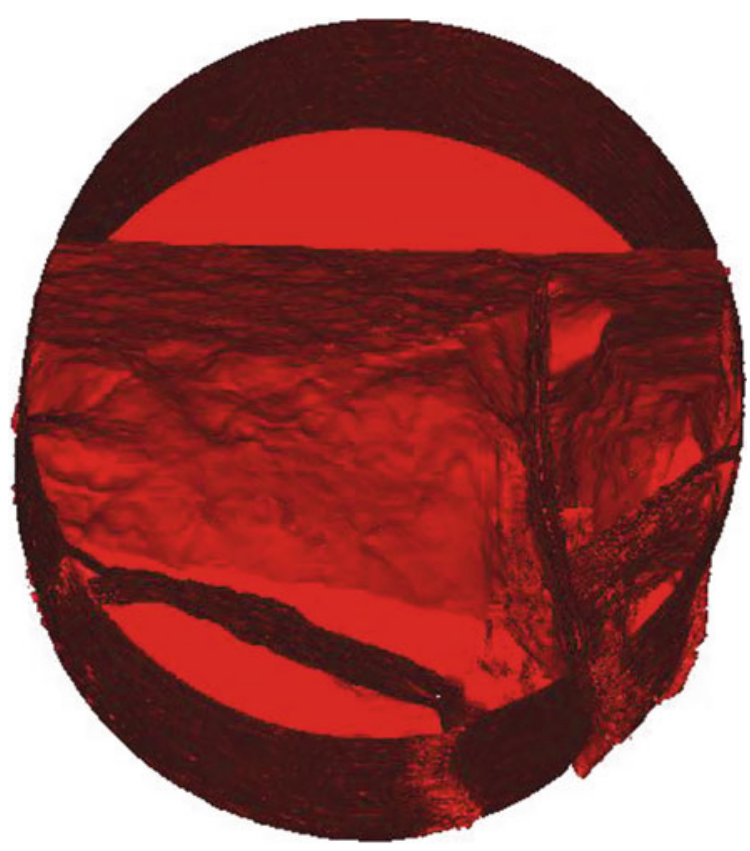

\subsubsection{Mesh Generation of Reconstructed Model}

3-MATIC is supporting of MIMICS software, and has a computer aided design (CAD) pretreatment tool and a pre-processing function of finite element model, it can help the triangular mesh optimization finite element analysis and fluid mechanics analysis, the model of local fairing and quality optimization becomes possible. The generated mesh was shown in Fig. 4.9.

The division of body mesh of model must be optimized. On the basis of generated surface mesh, the model body was divided by the way of continuous rock. The body mesh was generated continuously in the cracks and matrix, and finally the three-dimensional finite element model was established. In this chapter, the reconstruction model was divided into grids; there were 500 thousand information units in the model. The subdivision of 3D volume mesh was shown in Fig. 4.10.

\subsubsection{Three-Dimensional Model Parameters}

MIMICS could assign different material properties to the finite element model, which was generated by $3 \mathrm{D}$ reconstruction according to the different gray values, and the cracks could be distinguished using ANSYS software. Only cracks and matrices were distributed in the model. Therefore, parameters could be settled.

Using the different colors and the gray value of kinds of material, the density and elastic modulus, Poisson's ratio of material was defined as the parameters of model 
Fig. 4.9 Model diagram of mesh after optimization

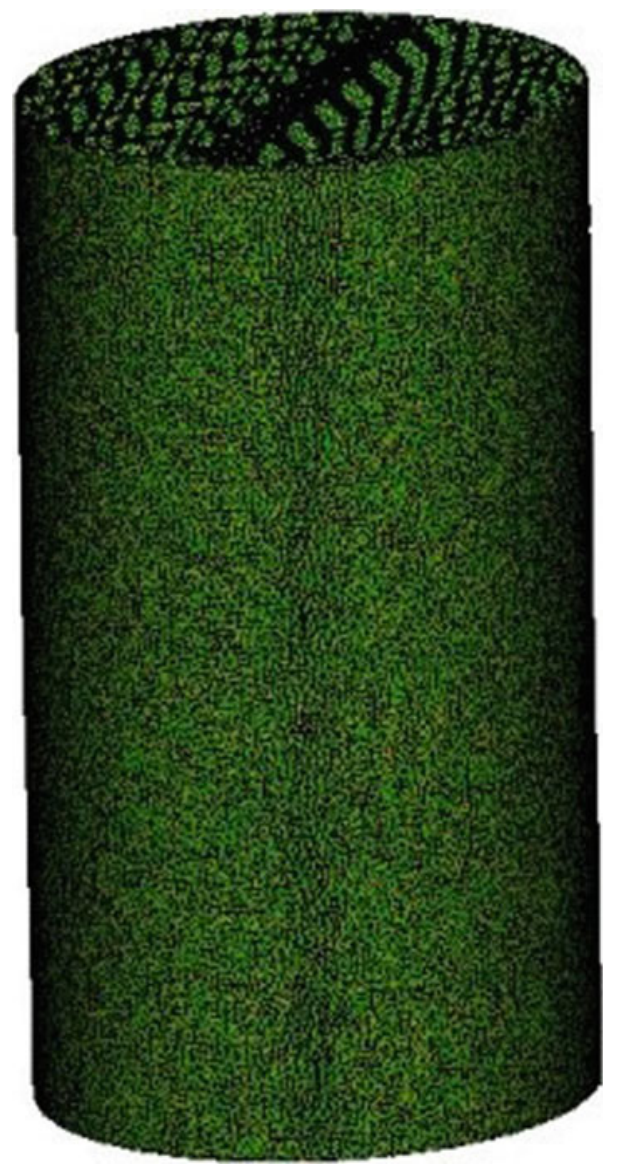

in ANSYS software. Therefore, this step required to definite of material were attributed to distinguish different substances. The process of defining the physi-mechanical parameters of each node unit in the three-dimensional model was shown in Fig. 4.11; the resulting fractured rock model was shown in Fig. 4.12.

\subsubsection{Numerical Simulation of Fissured Rock}

This model was derived for the ANSYS format. In this section, this model could be analyzed and calculated using the grid transformation tool to import into FLAC3D software. Numerical simulation results of three-dimensional fissured rock model could be obtained under uniaxial cyclic load/unload, and the influence of heterogeneity on microstructure of sandstone specimens macrocharacters could be analyzed. The simulation results and actual test results were compared and analyzed in the three dimensional reconstruction model of rock. 
Fig. 4.10 The internal structure of body grid in three-dimensional finite element model

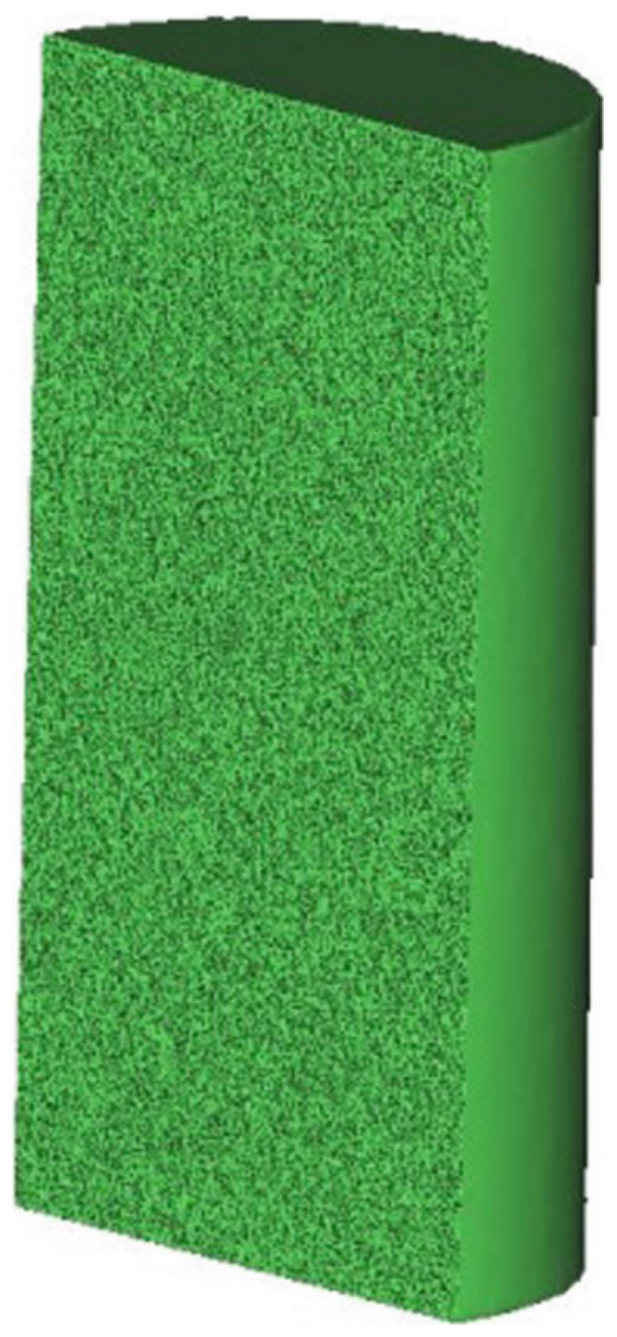

\subsubsection{Selection of Material Parameters}

The model was imported into FLAC3D, in order to avoid the contact problem that internal cracks of sandstone was empty under loading, and reduced the error of numerical simulation results, the fracture was treated as a virtual material, it was not the strength. Therefore, the modulus of elasticity and cohesion of sandstone were less small, the remaining sandstone matrix were uniformly given material parameters (Table 4.6 and Fig. 4.13). 


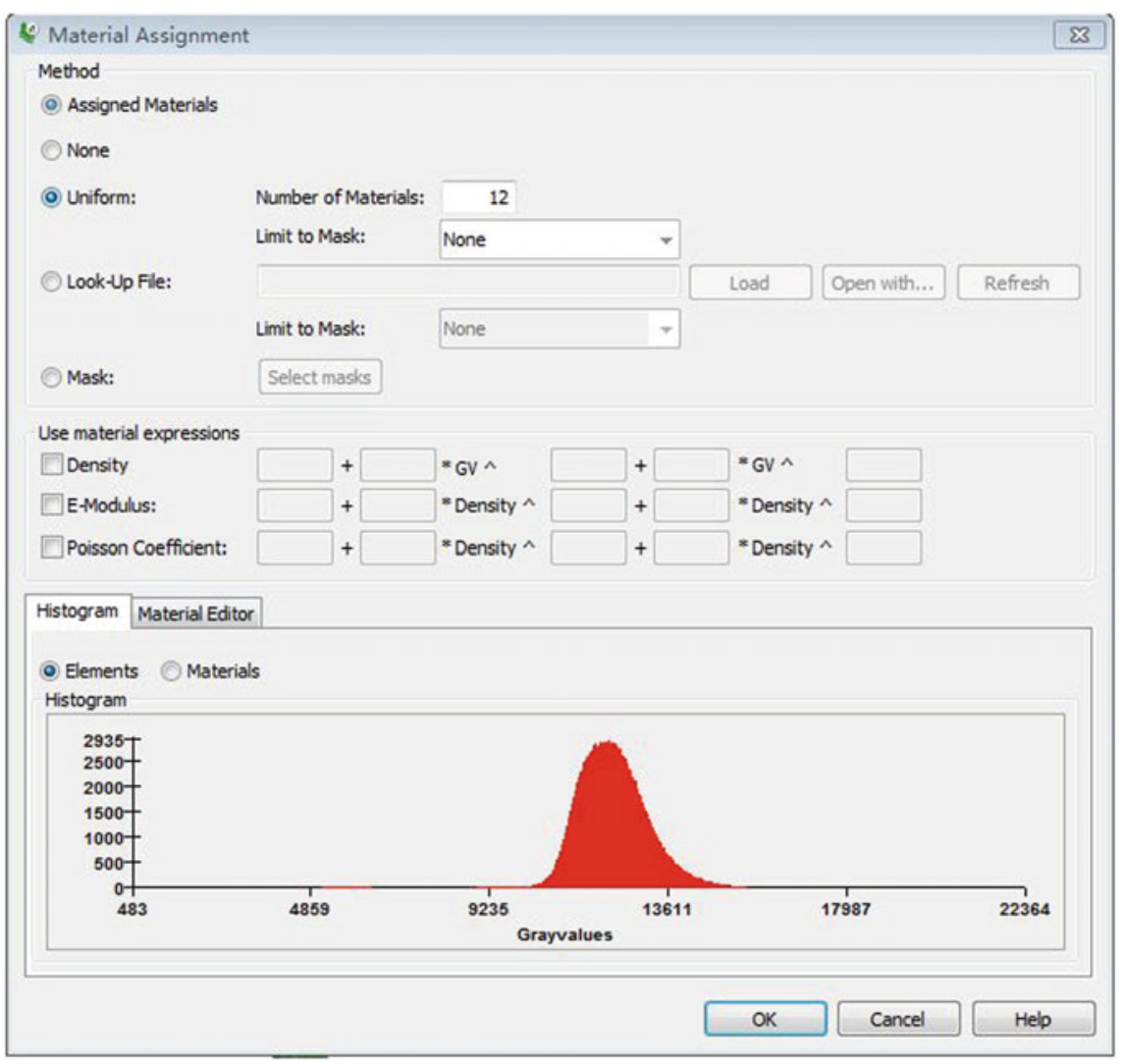

Fig. 4.11 The given different materials of model after the grid partition

\subsubsection{Numerical Analysis of Model}

As shown in Figs. 4.14 and 4.15, in the first stage of cyclic load/unload, numerical simulation results were coinciding with the experimental results, the simulation results of stress-strain curve and actual stress-strain curve was relatively consistent; Results of numerical simulation under load/unload were difference with the actual test results in the second phase under cycle load/unload, this maybe due to the actual test for rock material composition in various, and there were many micro holes and the number of cracks result in the different and the irreversible plastic with the actual test in two level cyclic load/unload process; There were differences between the final failure and the actual situation of damage stage, the specimen surface was not smooth. Due to the 3D reconstruction of rock under uneven surface, the process of displacement loading and the actual process were not consistent.

As shown in Figs. 4.16 and 4.17, the stress concentration of sandstone 3D model appeared in the cracks, and the stress concentration in the fracture deflection was more 

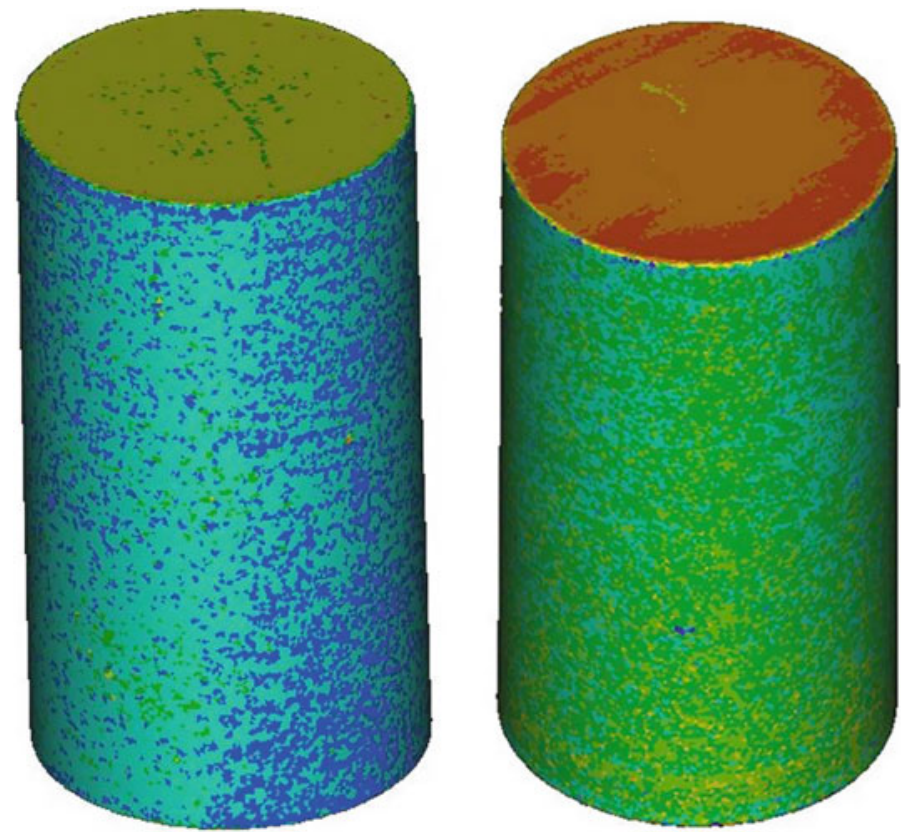

Fig. 4.12 Fissure body models of roof and floor sandstone

Table 4.6 The selection of material parameters of fissured rockmass model in roof and floor sandstone

\begin{tabular}{l|l|l|l}
\hline Media type & Poisson's ratio & Cohesion $(\mathrm{MPa})$ & Internal friction angles $\left(^{\circ}\right)$ \\
\hline Matrix of roof sandstone & 0.26 & 15.81 & 32 \\
\hline Matrix of floor sandstone & 0.25 & 11.92 & 60 \\
\hline Fissure & 0.2 & 0.16 & 40 \\
\hline
\end{tabular}
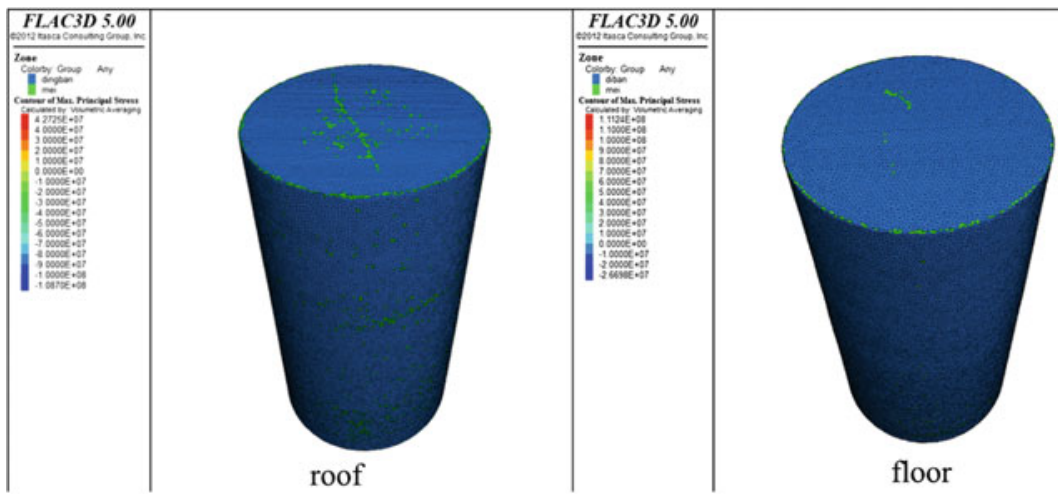

Fig. 4.13 Numerical models of roof and floor sandstone 


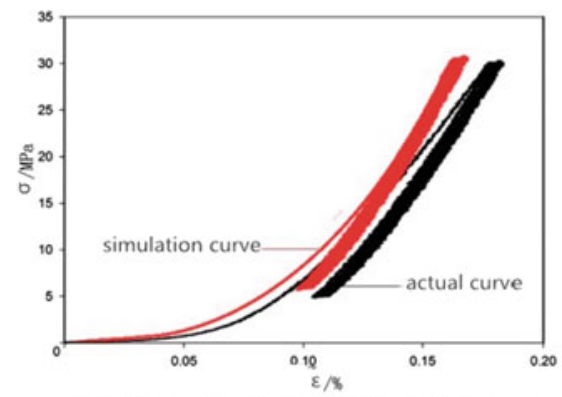

(a) The first stage under cyclic loading and unloading

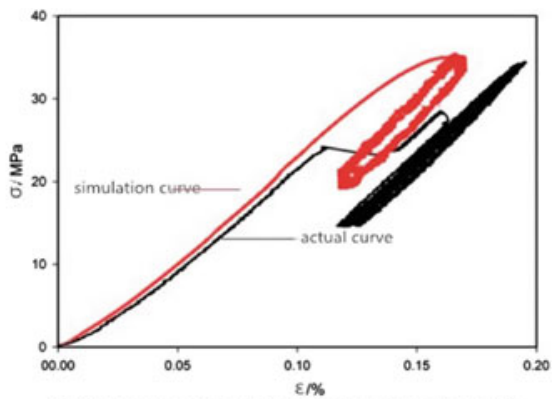

(b) The second stage under cyclic loading and unloading

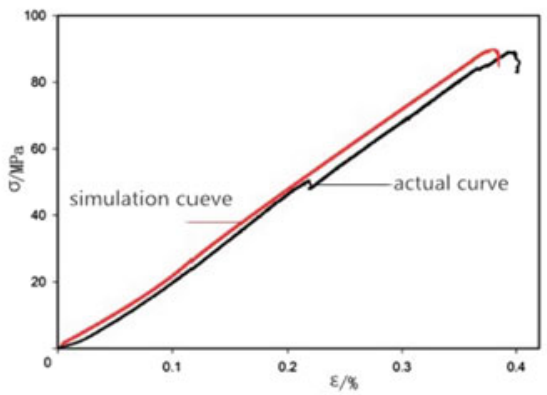

(c) The failure stage under uniaxial loading

Fig. 4.14 Stress-strain comparison charts of roof

obvious. The stress concentration area of these cracks had the tendency to extend to the interior of sandstone matrix; In the early stage of loading, the concentrated stress degree was low because of small load, and the stress concentration was only at the end of cracks, and no obvious failure unit appears; With the increase of stress, many microcracks were generated. With the increase of stress, microcracks would rapidly expand until the whole specimen failure.

The above analysis showed that the macro mechanical properties of sandstone were affected by their internal cracks and spatial distribution. With the increase of stress, the sandstone produced uncoordinated deformation at the cracks, which could result in stress concentration in matrix near cracks, especially at the cracks tip. The stress concentration area were presented the trend to the development of rock samples, the discontinuous small cracks were met and merged, large macro main cracks were formed, and the sandstone samples were failure due to the cracks propagation and coalescence.

In this chapter, numerical simulation results of sandstone samples were selected to analyze the mechanical mechanism of rock failure. As shown in Fig. 4.18, numerical simulations of plastic elements were comprised in the process of change. With the increasing stress, the status model unit of rock failure was also change, the plastic unit reached shear-n shear-p under the action of stress, and the plastic zone was completely through in the failure stage. Taking the red section of compaction 


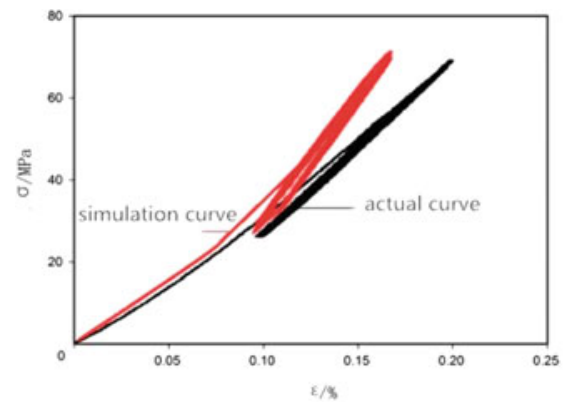

(a) The first stageunder cyclic loading and unloading

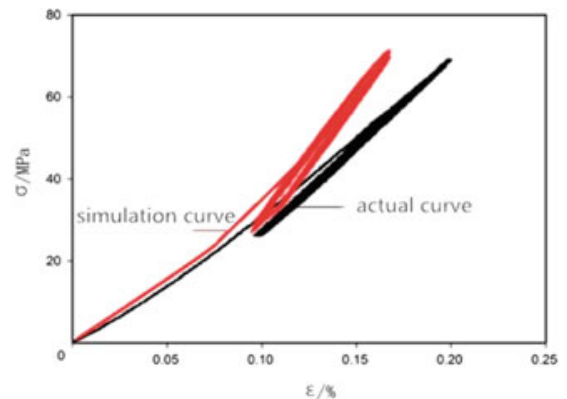

(b) The second stage under cyclic loading and unloading

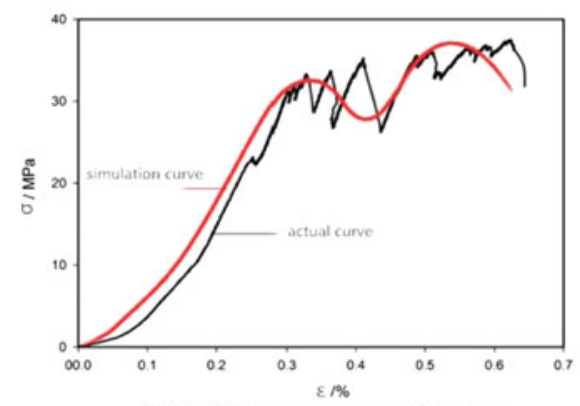

(c) The failure stage under uniaxial loading

Fig. 4.15 Stress-strain comparison charts of floor sandstone specimen

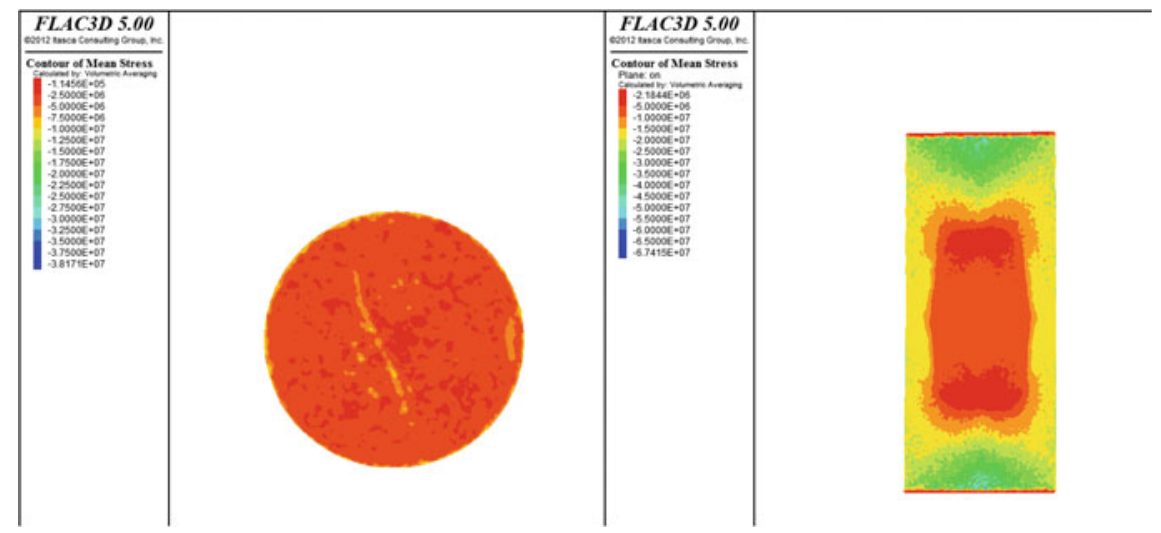

Fig. 4.16 Stress profile of fissures model the roof sandstone

stage as an example, it showed that shear failure was occurring in the shear-n shear-p red plastic unit status at the compaction stage; With the increase of stress, the plastic unit in the first stage of load/unload in the region was shear-p in blue; As the stress continues to increase, the plastic unit in the second stage of load/unload 


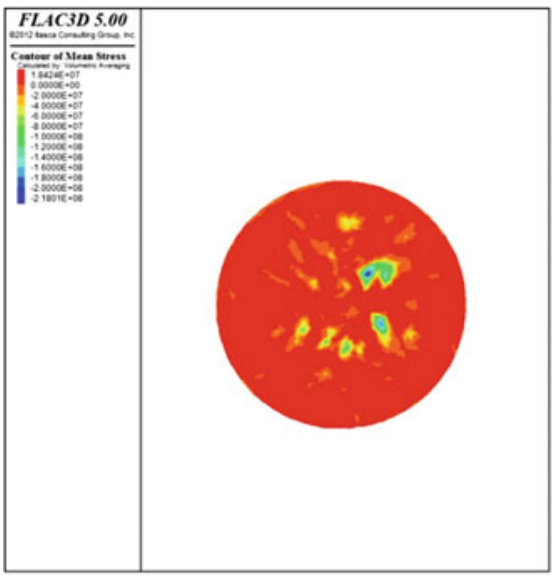

(a) stress distribution of cross - section

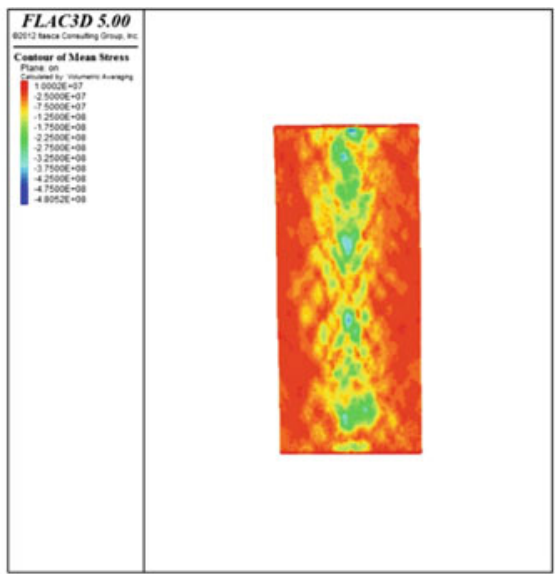

(b) stress distribution of Longitudinal profile

Fig. 4.17 Stress profile of fissures model of bottom sandstone

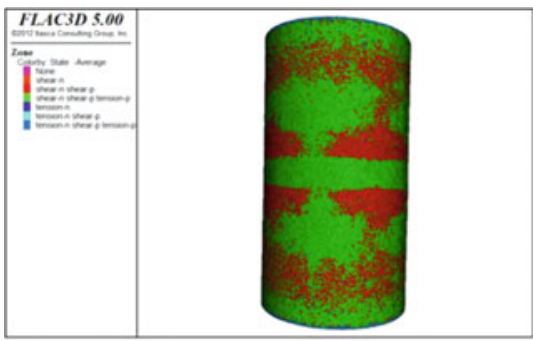

(a) Compression stage

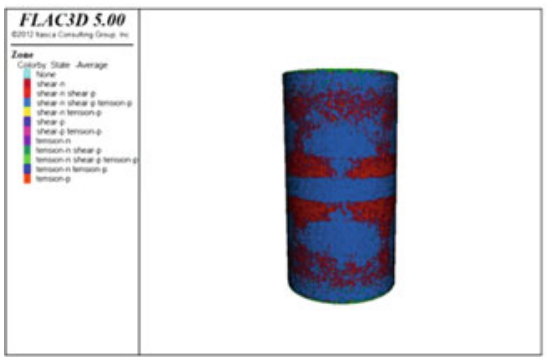

(c) The second stage under cyclic load/unload

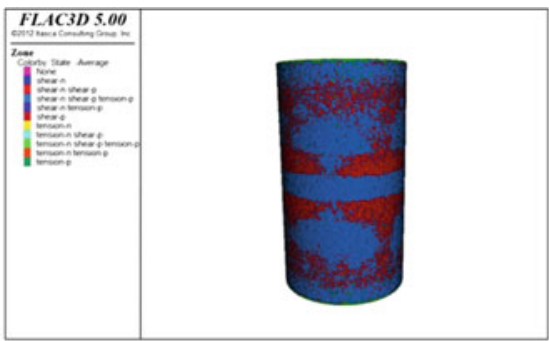

(b) The first stageunder cyclic load/unload

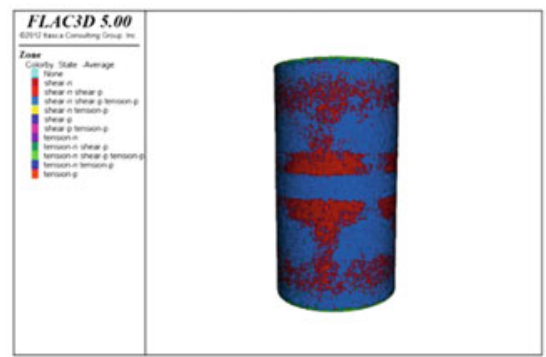

(d) The failure stage under cyclic load/unload

Fig. 4.18 Distribution of plastic units during loading

became tension-n shear-p, and at this stage tensile failure occurs due to Poisson effect; Prior to this, the plastic units only appeared in the local region and did not have a larger range of connectivity. In the failure stage, this region was tension-p 


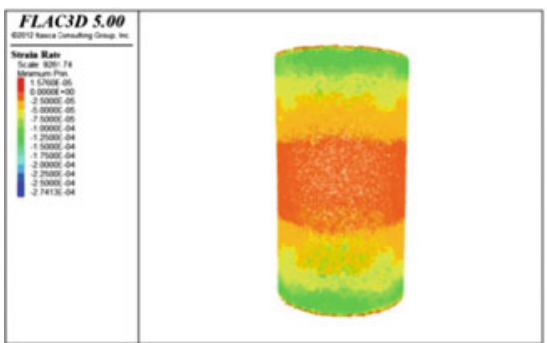

(a) Compression stage

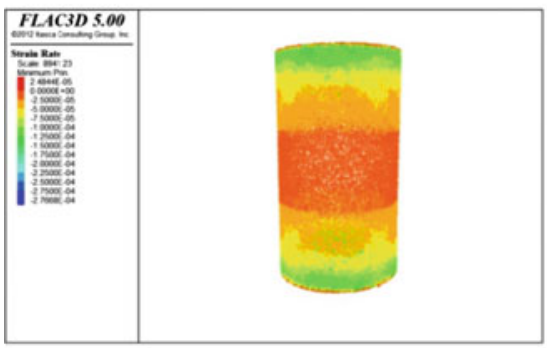

(c) The second stage under cyclic load/unload

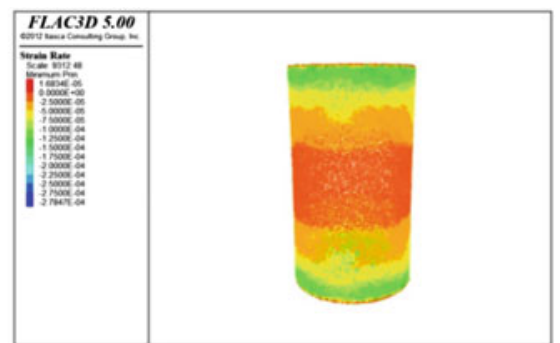

(b) The first stageunder cyclic load unload

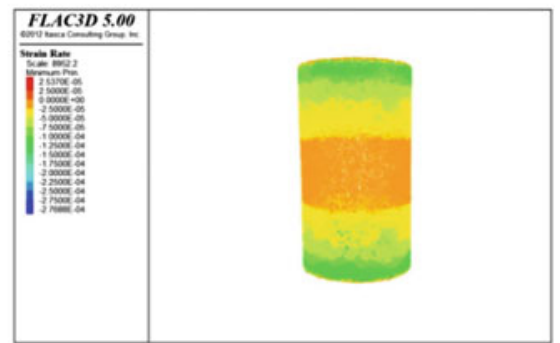

(d) The failure stage under cyclic load/unload

Fig. 4.19 Strain rate of surface during loading

status and tensile failure occurs. This result was consistent with the actual macro failure characteristics.

As shown in Fig. 4.19, it was the strain rate change model in the process of loading. With the increase of stress concentration, the former three stages had different degrees of high strain rate; the strain rate of high concentration would be connected to the local stress concentration zone. With the increasing of stress, the strain rate performance characteristics were from low to high. After the second stage of cyclic load/unload, the local high strain rate concentration zones were met with each other and eventually form a plastic zone at the failure stage.

\section{References}

Argandoña VGRD, Rey AR, Celorio C et al (1999) Characterization by computed X-ray tomography of the evolution of the pore structure of a dolomite rock during freeze-thaw cyclic tests. Phys Chem Earth Part A 24(7):633-637

Bornert M (2010) X-ray micro CT for studying strain localization in clay rocks under triaxial compression. Adv X-ray Tomogr Geomater 118:35

Chen S, Yue ZQ, Tham LG (2007) Digital image based approach for three-dimensional mechanical analysis of heterogeneous rocks. Rock Mech Rock Eng 40(2):145

Feng XT, Chen S, Zhou H (2004) Real-time computerized tomography (CT) experiments on sandstone damage evolution during triaxial compression with chemical corrosion. Int J Rock Mech Min Sci 41(2):181-192 
Kawakata H, Cho A, Kiyama T et al (1999) Three-dimensional observations of faulting process in Westerly granite under uniaxial and triaxial conditions by X-ray CT scan. Tectonophysics 313 (3):293-305

Kawakata H, Cho A, Yanagidani T et al (2000) Gross structure of a fault during its formation process in Westerly granite. Tectonophysics 323(1):61-76

Louis L, Wong T, Baud P et al (2006) Imaging strain localization by X-ray computed tomography: discrete compaction bands in Diemelstadt sandstone. J Struct Geol 28(5):762-775

Nasseri MHB, Rezanezhad F, Young RP (2011) Analysis of fracture damage zone in anisotropic granitic rock using 3D X-ray CT scanning techniques. Int J Fract 168(1):1-13

Ueta K, Tani K, Kato T (2000) Computerized X-ray tomography analysis of three-dimensional fault geometries in basement-induced wrench faulting. Eng Geol 56(1):197-210

Viggiani G, Lenoir N, Bésuelle P et al (2004) X-ray micro tomography for studying localized deformation in fine-grained geomaterials under triaxial compression. C R Méc 332(10):819-826

Wang LB, Frost JD, Voyiadjis GZ et al (2003) Quantification of damage parameters using X-ray tomography images. Mech Mater 35(8):777-790

Open Access This chapter is licensed under the terms of the Creative Commons Attribution-NonCommercial 4.0 International License (http://creativecommons.org/licenses/by-nc/ $4.0 /$ ), which permits any noncommercial use, sharing, adaptation, distribution and reproduction in any medium or format, as long as you give appropriate credit to the original author(s) and the source, provide a link to the Creative Commons license and indicate if changes were made.

The images or other third party material in this book are included in the book's Creative Commons license, unless indicated otherwise in a credit line to the material. If material is not included in the book's Creative Commons license and your intended use is not permitted by statutory regulation or exceeds the permitted use, you will need to obtain permission directly from the copyright holder.

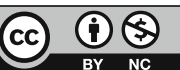




\section{Chapter 5 \\ Experimental Investigation on Nonlinear \\ Dynamic Evolution Patterns of Cracks \\ in Rock Failure Process}

\subsection{Introduction}

Based on CT scanning results of sandstone, CT images were analyzed using the MATLAB software and the evolution pattern of mesoscopic cracks were explored under uniaxial cyclic load/unload. Fractal dimension was used to define the degree of chaos in the process of cracks propagation. System entropy model was established using the fractal dimension. Verifying the relationship between the areas and lengths of cracks and stress, a crack growth factor model was proposed. It revealed the nonlinear dynamic evolution patterns of cracks propagation in the process of sandstone failure using CT parameters. This model provided a new method for predicitng rock dynamic hazard. Compared with the actual test results using the three-dimensional reconstruction and numerical simulation of roof and bottom sandstone's mesostructure of initial stage, the correctness of numerical simulation was verified.

The rock failure process does not occur in a short time, it is a significant accumulation process, which is determined by the brittle characteristics of rock. In the process of cyclic load/unload; the higher concentrated stress will appear at the tip of rock cracks, which will lead to the initiation and expansion of cracks inside the rock, and gradually through the new macroscopic cracks leading to rock failure.

Most scholars studied the cracks evolution using the similar model test, and the process of cracks initiation, expansion and penetration failure could be observed intuitively and clearly. Nolen-Hoeksema and Gordon (1987) conducted a compression test on the marble with prefabricated sloping fissures. Through the observation of cracks tip and the failure mode, it showed that the propagation process of cracks was asymmetric, and the damage inside the rock could be predicted using the observation of cracks distribution on the specimen's surface. Many scholars studied the evolution pattern of cracks on the two-dimensional plane using a microscope, and the data information of microstructure of rock was obtained. Wong (1982), and Wong et al. (2004) obtained that the microstructure of granite 
crack was changed. The rapid development of CT provided a variety of simple and effective methods for statistical analysis of data. Priest and Hudson (1976, 1979) acquired the geometrical characteristics of fractured rockmass. Reyes and Einstein (1991) observed the evolution of gypsum specimen under uniaxial static loading, the failure mechanism of fractured body was controlled using the geometric distribution of initial prefabricated cracks defects. Shen et al. (1995), Shen and Barton (1997) proposed new failure criteria, and improved the Griffith criterion, by which the test results could be explained reasonably. Bobet (2000) carried out the gypsum model test with the distribution of cracks defects under uniaxial loading, and the initiation, expansion and penetration mechanism of overlapping and non-overlapping distributions were obtained. Hoxha et al. (2005) concluded that the extended form of secondary cracks was significantly affected by the change in prefabricated cracks location.

In this chapter, CT scanning technology and digital image processing technology were used to obtain the cracks image under different load levels. The nonlinear dynamic model of cracks propagation evolution was revealed, and the nonlinear dynamic model of cracks propagation was established. At present, the evolution patterns of cracks propagation still stays in the whole process of cracks initiation-extension-failure of microscopic level, and does not explain the macroscopic failure pattern of rock through the process of cracks propagation. a three-dimensional visualization model of cracks was established, and a crack growth factor model was proposed, which provided a new way to study the transition from microscopic scale to macroscopic scale.

\subsection{Pattern of Nonlinear Dynamics Evolution of Rock Cracks}

It is necessary to study the microscopic cracks changes of rocks in order to explain the macroscopic mechanical properties of geomaterials. The complexity and heterogeneity of rock cracks and the nonlinear characteristics of its high degree of disordered distribution was constituted in rock failure process (Lin et al. 2000; Wong et al. 2001). Complex materials were previously difficult to quantitatively describe by fractal or chaotic methods. In this chapter, the fractal dimension of cracks in the two-dimensional plane was obtained, and the fractal dimension was used to analyze the sandstone cracks. The entropy model of rock system was established based on the fractal dimension, the mesoscopic mechanics of cracks was reveals from initiation and propagation. The cracks growth factor model was established using the deduction of cracks chaos discrimination, this model can reveal the expansion and evolution of sandstone cracks. 


\subsubsection{Fractal Theory}

The concept of fractal was first proposed by American mathematician B.B. Mandelbort. The Latin "Fractus" was the earliest source of fractal, and all irregular movements could be described by fractal in Mandelbrot's theory (Mandelbrot and Pignoni 1983; Mandelbrot 1977). Fractal geometry is a rapid new development discipline in recent years; it can describe any disorder movement. Fractional dimension is an important concept of fractal geometry, and its value can be a fraction. Fractal dimension became fractal geometry with the continuous development of society. It was pointed out that fractal dimension was a powerful tool for studying the patterns of most physics, and the treatment of those very irregular shapes must rely on the theory of fractal geometry (Goldberger et al. 2002; Faloutsos and Gaede 1996). The irregular, rough profile of rock fracture surface could be seen as self-similarity. Heping $\mathrm{X}$ et al. applied new geometric-fractal geometry to describe this irregularity. Fractal model of intergranular fracture was established, and the composite fracture between particles and intergranular was obtained. Fractal characteristics of ruptured rock surface were acquired using electron scanning and optical fractal analysis. Finally, the relationship between fractal dimension and macroscopic mechanical parameters of rock fracture was obtained (Xie and Chen. 1988, Heping X. 1989).

Fractal geometry mainly has two parts: the linear fractal and the nonlinear fractal (Mandelbrot 1999). The linear fractal theory uses some irregular curves with self-similar as the research object, and the fractal dimension is the continuous view of its basic view. The nonlinear fractal theory takes the geometries under the nonuniform linear transformation groups and the geometric shapes under the nonlinear transformation groups as the object of their properties. In the nonlinear fractal theory, the fractal dimension of nonuniform linear transformation reflects the essential characteristics of richness and complexity of nature. In this chapter, using the fractal dimension in the process of change under uniaxial cyclic load/unload, the evolution of cracks was also seemingly chaotic, it is necessary to quantitatively describe the expansion and evolution of cracks for realizing the disorder cracks propagation.

\subsubsection{Results Analysis on Fractal Dimension of Rock Cracks}

\subsubsection{Calculated Procedure of Fractal Box Dimension}

With the application and development of fractal geometry theory, the definition and measurement methods of fractal dimension are more abundant. There is no uniform definition of fractal dimension; the fractal dimension and the choice of measurement 
methods are discriminative for different research objects. There are six fractal dimensions, such as perimeter-area relation, power law spectrum, self-affine fractal method, box counting method, modified yardstick method. Based on the definition of box dimension, the fractal dimension of box dimension was used to calculate the fractal dimension in the process of rock cracks. Box-dimension method ( $\mathrm{Li}$ et al. 2009; Foroutan-pour et al. 1999) was also called Box method, network coverage, or coverage method. Using a square or a radius of epsilon (called a box) to cover the surface or curve of an object, and the number of boxes could alter as the epsilon changes. If the measured objects have fractal properties, the following relationships are satisfied:

$$
\mathrm{N}(\varepsilon)=a \varepsilon^{-D}
$$

where $a$ is a constant. Taking the logarithm of both sides, it can be described as follow:

$$
\mathrm{D}=\lim _{\varepsilon \rightarrow 0} \frac{\ln (N(\varepsilon) / a)}{\ln (1 / \varepsilon)}
$$

a set of data $\mathrm{N}(\varepsilon)-\varepsilon$ could be gotten by covering, the double logarithmic graph could be drawn, the scale-free region on the $\ln \varepsilon-\ln N$ graph could be identified. Using the least square method, the slope was obtained, and the absolute value of slope was the fractal dimension value.

The specific steps of cracks fractal dimension were calculated using the box counting method.

1. For rock cracks image of a pixel-sized $M \times M$, it was found as a three-dimensional surface. The length and width are $M$, the height is $L$, using the pixel series of image to represent, it is set to $L=256$.

2. The cracks plane image $(M \times M)$ was divided into a grid of size, and the coordinate scale of "height" was divided $R \times R$ equally, the unit of division was $R \times L / M$. Therefore, the three-dimensional curved surface was divided into many three-dimensional "boxes". The key of this division method had the same division "number" of width and height direction.

3. Within each the grid was divided into the size of $R \times R$, the pixel value $u$ and pixel minimum value $b$ was founded. The minimum to the maximum pixel was calculated, pixels how many boxes to cover, box number to remember $n(i, j)$, assuming that now the grid was $(i, j)$. That was $n(i, j)=[(u-b+R-1) / R]$, in the equation for the whole symbol.

4. Take the sum of each box $R \times R$, Counted as $N$, that was $N=\operatorname{sum}(n(i, j))$.

5. In theory, the fractal dimension at this time was $D=-\log N / \log R, R$ was close to infinity. $R$ was limited in reality. Therefore, the sizes of $R$ value were change, a group $N$ was found, and then using the linear fitting method, it showed that the slope of straight line was the required fractal dimension $D$. 


\subsubsection{Results Analysis on Fractal Dimension of Sandstone Cracks}

With good self-similarity of surface cracks in the sandstone, the cracks distribution in the sandstone was handled by the box counting method of fractal geometry using the two-dimensional CT scanning algorithm. The calculation results were shown in Tables 5.1, 5.2 and Figs. 5.1, 5.2.

As shown in Figs. 5.1 and 5.2, fractal dimension curve showed as follow:

1. In the initial unloaded status, the roof and bottom sandstone samples were scanned, and the fractal dimensions of layers were different. Therefore, the initial damage of sandstone was not uniform, and it was an initial damage medium mainly based on the existence of microcracks.

2. As shown in Figs. 5.1 and 5.2, the evolution of fractal dimension in this process was divided into three stages. The first stage was the unloading stage - the ends of the first stage of the cycle load/unload. With the stress increases, the cracks begin to expand, the complexity of cracks structure and the fractal dimension increases. The second stage was the end of the first stage of cycle and unloading to the end of the second stage of cyclic load/unload, fractal dimension of different layers was almost the same. However, fractal dimension of the 650 layer slice was almost unchanged because the initiation of cracks was continued to meet at this stage, the overall shape and structure of cracks were from the complex to simple, and the fractal dimension stabilization was occured. At the other level, due to the presence of new cracks around the through cracks, the complexity of cracks structure and the fractal dimension increased. The third stage was the end of the second stage of cycle load/unload to the specimen

Table 5.1 Calculated results of fractal dimensions of roof sandstone

\begin{tabular}{l|l|l}
\hline Scan phase & Scan sections & Fractal dimensions \\
\hline \multirow{2}{*}{ Before loading $(0 \mathrm{MPa})$} & 350 layer & 1.8769 \\
\cline { 2 - 3 } & 450 layer & 1.8767 \\
\cline { 2 - 3 } & 550 layer & 1.8767 \\
\cline { 2 - 3 } After the first phase $(9 \mathrm{MPa})$ & 650 layer & 1.8768 \\
\hline \multirow{2}{*}{ After the second phase $(27 \mathrm{MPa})$} & 350 layer & 1.8789 \\
\hline & 450 layer & 1.8784 \\
\cline { 2 - 3 } & 550 layer & 1.8787 \\
\cline { 2 - 3 } & 650 layer & 1.8792 \\
\hline \multirow{2}{*}{ After failure $(81.9 \mathrm{MPa})$} & 350 layer & 1.8792 \\
\cline { 2 - 3 } & 450 layer & 1.8787 \\
\cline { 2 - 3 } & 550 layer & 1.8789 \\
\cline { 2 - 3 } & 650 layer & 1.8796 \\
\hline & 350 layer & 1.8764 \\
\hline & 450 layer & 1.8765 \\
\cline { 2 - 3 } & 550 layer & 1.8765 \\
\hline & 650 layer & 1.8766 \\
\hline
\end{tabular}


Table 5.2 Calculated results of fractal dimensions of bottom sandstone

\begin{tabular}{|c|c|c|}
\hline Scan phase & Scan sections & Fractal dimensions \\
\hline \multirow[t]{4}{*}{ Before loading (0 MPa) } & 350 layer & 1.8759 \\
\hline & 450 layer & 1.8757 \\
\hline & 550 layer & 1.8760 \\
\hline & 650 layer & 1.8758 \\
\hline \multirow[t]{4}{*}{ After the first phase (6 MPa) } & 350 layer & 1.8767 \\
\hline & 450 layer & 1.8765 \\
\hline & 550 layer & 1.8767 \\
\hline & 650 layer & 1.8769 \\
\hline \multirow[t]{4}{*}{ After the second phase (18 MPa) } & 350 layer & 1.8769 \\
\hline & 450 layer & 1.8769 \\
\hline & 550 layer & 1.8771 \\
\hline & 650 layer & 1.8770 \\
\hline \multirow[t]{4}{*}{ After failure (37.5 MPa) } & 350 layer & 1.8754 \\
\hline & 450 layer & 1.8755 \\
\hline & 550 layer & 1.8756 \\
\hline & 650 layer & 1.8755 \\
\hline
\end{tabular}

Fig. 5.1 The tendency of fractal dimensions at each layer of roof sandstone on different stress conditions

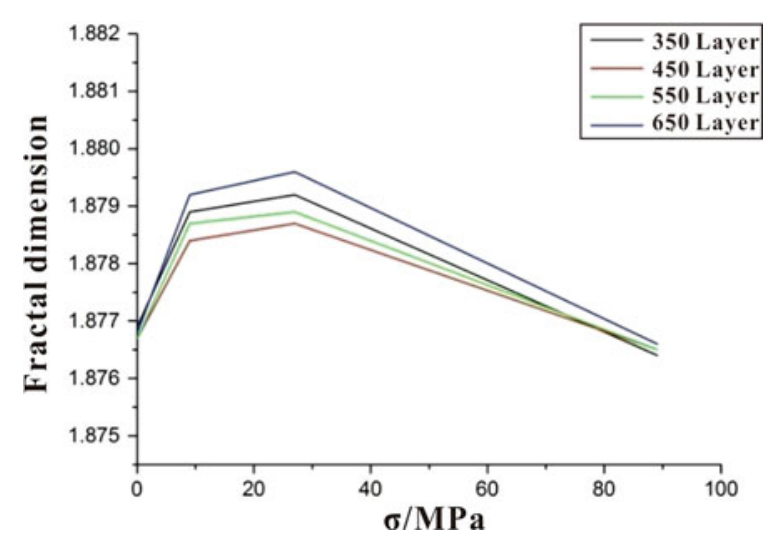

destruction. At this stage, the cracks were intermittent to accelerate, and many macroscopic main cracks appeared. The failure surface was formed in the crack-dense area, and the specimens were cracked along the failure surface. However, under the stress, the non-main cracks gradually were closed, and the shape and structure of cracks were gradually developed toward the simplification resulting in the fractal dimension decreasing rapidly.

3. Based on the analysis and calculation of fractal dimension of cracks at each scanning level, it was concluded that the propagation and evolution of cracks in the rock failure process, they were quantitatively described by the fractal dimension, that is to say, the damage degree of rock was quantitatively described by the size of fractal dimension. 
Fig. 5.2 The tendency of fractal dimensions at each layer of bottom sandstone at different stress conditions

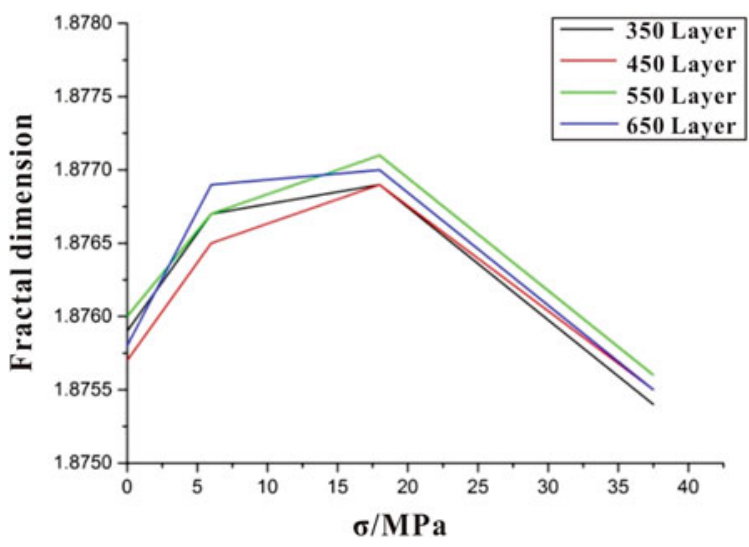

\subsubsection{Entropy Model of Rock System Based on Fractal Dimension}

The entropy can measure the disorder degree and the entropy of a large number of microscopic particles in the system from the microscopic point of view, and the entropy increases with the disorder degree and the chaotic status of the granular matter in the system. The chaos of system is proportional to the entropy of system. This relationship is first established by Boltzmann, called the Boltzmann theorem, as follow:

$$
S=k \times \ln W
$$

where $S$ is the entropy, the description of system mechanical status; $k$ is the Boltzmann constant; $W$ is the chaos of system.

Therefore, if the chaos degree of rock system is defined, the entropy of rock system $S$ can be revealed, and the meso-structure evolution of rock failure process can be verified from disorder to order.

The more the microscopic state of system, the greater the degree of confusion. The system could be quantified with the number of micro-state quantitative description. Since the fractal dimension can express the complexity of system (i.e., the degree of chaos), the fractal dimension of sandstone cracks was calculated under cyclic load/ unload. The microcracks began to expand and gradually converged into cracks, this extension and the process of cracks distribution showed a trend of reducing the dimension of system from the disorder to the orderly dynamic characteristics. Therefore, the fractal dimension was used to define the degree of confusion $W$.

The Gauss Amp function expression is:

$$
y=y_{0}+A \mathrm{e}^{-\frac{\left(x-x_{c}\right)^{2}}{2 \omega^{2}}}
$$


where $y_{0}$ is the offset of $y$-axis direction of curve, and $A$ is the difference between the maximum and minimum values of curve, that is the amplitude of function. The $A \mathrm{e}^{-\frac{\left(x-x_{c}\right)^{2}}{2 \omega^{2}}}$ can be defined as a normal distribution of deformation, the standard normal distribution formula: $f(x)=\frac{1}{\sqrt{2 \pi} \sigma} \mathrm{e}^{-\frac{(x-\mu)^{2}}{2 \sigma^{2}}}$, the symmetry axis is $x_{c}, \omega$ for the relevant parameters to meet $2 \omega=F W H M / \operatorname{sqrt}(\ln (4))$, according to the equation to calculate its size.

The function is symmetrical about $x=\mathrm{x}_{c}$, and when $x=\mathrm{x}_{c}$ the extremes are obtained, If $A>0$, the maximum value is obtained at that point; if $A<0$, the minimum value is obtained at that point. The distribution of function is basically similar to the normal distribution, when $A>0$, the opening goes down; $A<0$, the opening is upward. The larger the $\omega$ value, the more the function image is "thin"; the smaller $\omega$, the more function image is "too thick".

As shown in Figs. 5.1 and 5.2, the fractal dimension of each section of roof and floor sandstone was changed and then fitted with the above function. Each parameter in the equation was given practical significance. The dependent variable $y$ is the fractal dimension $D, x_{c}$ is the lower limit stress $\sigma_{\mathrm{c}}$ of the secondary cyclic load/unload, $y_{0}$ is the fractal dimension value $D_{0}$ when the stress is $\sigma_{0}, A$ is the fluctuation range of fractal dimension value, and $\omega$ is the relevant parameter. Figures 5.3 and 5.4 showed the fitting parameters. Table 5.3 showed the relevant parameters.

As shown in Table 5.3, the fractal dimension of top and bottom sandstone was in accordance with the distribution of Gauss Amp function in the process of cracks propagation. Hence, the evolution formula of and stone crack propagation based on fractal dimension was obtained as follows:

$$
D=D_{0}+A \mathrm{e}^{-\frac{\left(\sigma-\sigma_{\mathrm{c}}\right)}{2 \times \omega}}
$$

Fig. 5.3 Fitting curves of fractal dimensions and stress curves of roof cracks

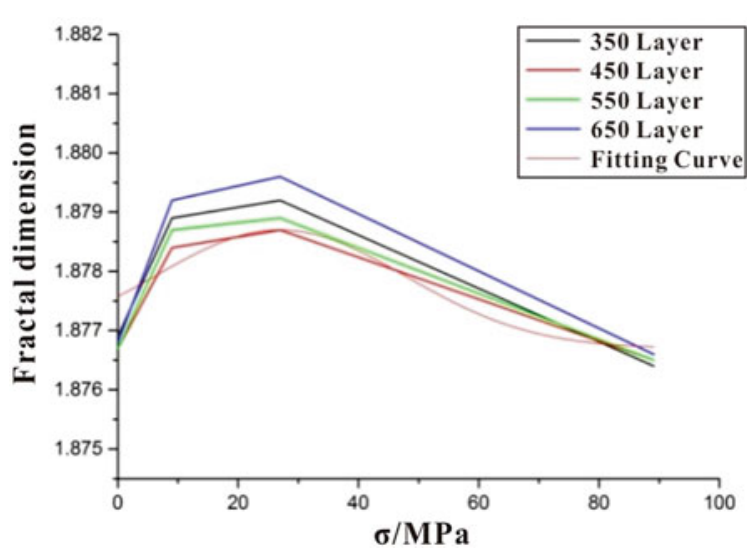


Fig. 5.4 Fitting curves of fractal dimensions and stress curves of floor cracks

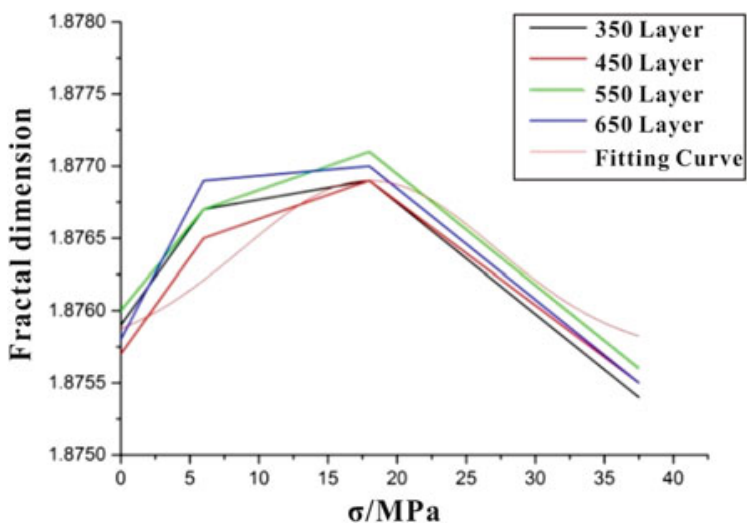

Table 5.3 Parameters and equations for fitting curves

\begin{tabular}{l|l|l|l|l|l}
\hline \multirow{2}{*}{ Rock type } & \multicolumn{4}{|l|}{ Related parameters } & \multirow{2}{*}{ Equations for fitting curves } \\
\cline { 2 - 5 } & $\mathrm{D}_{0}$ & $\mathrm{~A}$ & $\sigma_{\mathrm{c}}$ & $\omega$ & \\
\hline Roof sandstone & 1.8767 & 0.002 & 27 & 20.97 & $D=1.8767+0.002 \mathrm{e}^{-\frac{(\sigma-27)^{2}}{2 \times 20.97}}$ \\
\hline Floor sandstone & 1.8759 & 0.003 & 18 & 8.72 & $D=1.8759+0.003 \mathrm{e}^{-\frac{(\sigma-18)^{2}}{2 \times 8.72}}$ \\
\hline
\end{tabular}

where the system chaos $W$ in Eq. (5.4) can be expressed by the fractal dimension of cracks. Therefore, it can put the formula (5.6) into the formula (5.4), the sandstone system entropy model is:

$$
S=\mathrm{k} \times \ln \left(D_{0}+A \mathrm{e}^{-\frac{\left(\sigma-\sigma_{\mathrm{c}}\right)}{2 \times \omega}}\right)
$$

where $k$ is a constant; $\sigma_{\mathrm{c}}$ is the lower limit stress value of the secondary cyclic load/ unload; $\sigma$ is the stress value at any time; $D_{0}$ is the fractal dimension value of initial state; $\omega$ is the coefficient in the Gauss Amp function; The range of volatility; $S$ is the system entropy of sandstone failure process.

This model quantitatively was described the evolution of system entropy in the process of sandstone cracks propagation and evolution, which provided a theoretical basis for the rock fracture. This experiment was only carried out CT scanning test on the top and bottom sandstone rock samples without other rocks test, but other rock samples may meet the model. Thus, the model could be extended to other hard rock rocks: $S=\mathrm{k} \times \ln \left(D_{0}+A \mathrm{e}^{-\frac{\left(\sigma-\sigma_{\mathrm{C}}\right)}{2 \times \omega}}\right)$, where the value of $\omega$ is related to the lithology of rock.

In this chapter, the entropy model of rock system combined the change of fractal dimension and the change of system entropy in the process of rock failure, the evolution of system entropy under the action of uniaxial cyclic load/unload was described quantitatively. It was proved that the dimensionality reduction, entropy reduction and order were unified in the process of rock failure. The dynamic 
characteristics of rock system from disorder to order could be described by the process of dimension reduction and entropy reduction. It was shown that the rock specimens were exhibited a critical behavior of destabilization as the stress increases during loading. The entropy of rock specimens and the fractal dimension of cracks were reduced, the distribution of cracks was ordered and the rock specimen was destabilized. The model described the evolution of rocks from microstructure to macroscopic failure.

\subsection{Chaotic Characteristics of Cracks Evolution in Rock Failure Process}

In nonlinear dynamics, the motion status of matter is deterministic and unpredictable, and this phenomenon is called chaos. Chaotic phenomenon is similar to the random motion and unpredictable. However, these research results show that chaos can be determined in the nonlinear dynamics, and the instability of motion status of material in the system leads to its unpredictability. With the continuous development of nonlinear dynamics, chaos is one of the most common motion status in nonlinear dynamics, and it is also the essential attribute of nonlinear dynamical system. In this chapter, the chaotic motion of cracks was derived from the nonlinear variation in the process of cracks propagation, and there was some kind of internal relation of certainty and randomness in the process of cracks change. Therefore, it is necessary to obtain the chaotic characteristics in the process of cracks propagation for predicting rockburst.

\subsubsection{Chaos Theory}

Chaotic motion has a certain non-linear feature, it is gradually converged to a certain point in the system of disorderly status-chaos, and there is no universally recognized mathematical definition. The chaos is defined as:

(Li-Yorke Chaos definition) Continuous mapping or point mapping $\mathrm{F}:[a, b] \times R \rightarrow[a, b],(\mathrm{x}, \lambda) \rightarrow F(x, \lambda)$ is chaos, If the following conditions are as follow:

1. There are periodic periods of all cycles;

2. There is a countless subset $\mathrm{S} \subset[a, b]$, where $S$ does not contain a periodic point:

(1) If any $x, y \in S$,

$$
\lim _{n \rightarrow \infty} \inf \left|F^{n}(x, \lambda)-F^{n}(y, \lambda)\right|=0
$$


(2) If any $x, y \in S(x \neq y)$,

$$
\lim _{n \rightarrow \infty} \sup \left|F^{n}(x, \lambda)-F^{n}(y, \lambda)\right|>0
$$

(3) If any $x \in S$ with $F$ is any periodic point,

$$
\lim _{n \rightarrow \infty} \sup \left|F^{n}(x, \lambda)-F^{n}(p, \lambda)\right|>0
$$

So far, chaos is the inherent law of seemingly disordered phenomena. Although there is no definite definition of chaos, it has mainly some common characteristics as follows: sensitivity to the initial value, intrinsic randomness, boundedness ergodicity, universality, self-similarity. The local area in the chaotic system appears to be complicated and disordered. However, the system is self-similar, and the self-similar structure is superimposed in the system, which makes the whole system seem to be chaotic and irregular.

\subsubsection{Discrimination of Chaotic Characteristics Defined by Li-Yorke}

This chapter uses the defined Li-Yorke criterion for cracks evolution, chaotic characteristics is derived assuming without any loading condition, the sandstone CT number for initial and with the increasing of stress cracks generated which can cause the new crack energy release. Therefore, the change process of describing the density of sandstone can be used to use trigger-growth-triggered cracks chain growth model. The density of microcrack was assumed, the generalized driving force was proportional to the production of cracks, and the CT number $n_{i+1}$ of sandstone can be expressed as: when the loading went to the stage of $i+1$.

$$
n_{i+1}=F_{1}(\sigma) \cdot n_{i}-F_{2}(\sigma) \cdot\left(N_{0}-n_{i}\right) \cdot X_{i}
$$

where

$N_{0}$ is total CT number of sandstone samples;

$\mathrm{X}_{i}=\frac{n_{i}}{N_{0}}$ dimensionless;

where $F_{1}(\sigma)$ is survival ratio of cracks, the quantitative description of cracks closure effect, no dimensionless scalar;

where $F_{2}(\sigma)$ is driving force depends on the magnitude of stress, the dimensionless scalar. 
According to the testing results of the whole process and the experimental results of literature, it can be assumed that:

$$
F_{2}(\sigma)=\frac{\ln \sigma}{\ln \sigma_{c}}
$$

where $\sigma_{\mathrm{c}}$ is uniaxial compression strength

Take the formula (5.11) into the formula (5.10):

$$
n_{i+1}=F_{1}(\sigma) \cdot n_{i}-\frac{\ln \sigma}{\ln \sigma_{c}} \cdot\left(N_{0}-n_{i}\right) \cdot \frac{n_{i}}{N_{0}}
$$

based on the formula (5.11):

$$
\begin{aligned}
X_{i+1} & =F_{1}(\sigma) \cdot X_{i}-F_{2}(\sigma) \cdot X_{i} \cdot\left(1-X_{i}\right) \\
& =F_{2}(\sigma) \cdot X_{i} \cdot\left[\frac{F_{1}(\sigma)-F_{2}(\sigma)}{F_{2}(\sigma)}+X_{i}\right]
\end{aligned}
$$

command:

$$
\begin{gathered}
\mathrm{x}_{\mathrm{i}}=\frac{\mathrm{F}_{2(\sigma)}}{\mathrm{F}_{1}(\sigma)-\mathrm{F}_{2(\sigma)}} \mathrm{x}_{\mathrm{i}} \\
\mathrm{F}_{2}(\sigma)=\frac{\left[\mathrm{F}_{1}(\sigma)-\mathrm{F}_{2(\sigma)}\right]^{2}}{\mathrm{~F}_{2(\sigma)}}
\end{gathered}
$$

where $F_{2}(\sigma)$ is the single valued function of $F_{1}(\sigma)$ and $F_{2}(\sigma)$, which is defined as the generalized driving force; It's the sum of single valued functions. $X_{i}$ is the single valued functions.

Based on the formulas (5.14) and (5.15), (5.10) and (5.14) can be expressed as:

$$
\mathrm{X}_{\mathrm{i}+1}^{2}=\mathrm{f}\left(\mathrm{F}_{2(\sigma)}, \mathrm{x}_{\mathrm{i}}\right)=\mathrm{F}_{2(\sigma)} \mathrm{x}_{\mathrm{i}}\left(1+\mathrm{x}_{\mathrm{i}}\right)
$$

The standard formula of Logistic:

$$
x_{n+1}=f\left(x_{n}, \mu\right)=\mu x_{n}\left(1+x_{n}\right)
$$

Equation (5.16) is a one-dimensional nonlinear mapping, which is a Logistic equation with chaotic characteristics of Logistic mapping. As shown in Tables 5.4 and 5.5, the theoretical calculation results of CT number of the top floor sandstone specimen are $0-6.27 \%$ and $0-7.66 \%$ respectively. Therefore, the evolution of sandstone cracks in the single axial cyclic loading condition has chaotic characteristics. 
Table 5.4 Theoretical calculation results of roof sandstone specimens are compared with experimental results

\begin{tabular}{|c|c|c|c|c|}
\hline The scanning stage & $\begin{array}{l}\text { Scanning } \\
\text { layer }\end{array}$ & $\begin{array}{l}\text { Experimental results } \\
\text { of CT numbers }\end{array}$ & $\begin{array}{l}\text { Computed results } \\
\text { of CT numbers }\end{array}$ & $\begin{array}{l}\text { Error } \\
(\%)\end{array}$ \\
\hline \multirow{4}{*}{$\begin{array}{l}\text { The stage of } \\
\text { unloading }\end{array}$} & 350 layer & 576.03 & & \\
\hline & 450 layer & 592.74 & & \\
\hline & 550 layer & 589.88 & & \\
\hline & 650 layer & 607.59 & & \\
\hline \multirow{4}{*}{$\begin{array}{l}\text { The end of the first } \\
\text { stage of loading }\end{array}$} & 350 layer & 610.61 & 572.32 & 6.27 \\
\hline & 450 layer & 614.75 & 589.87 & 4.05 \\
\hline & 550 layer & 606.70 & 575.35 & 5.17 \\
\hline & 650 layer & 611.07 & 606.02 & 0.83 \\
\hline \multirow{4}{*}{$\begin{array}{l}\text { The end of the second } \\
\text { stage of loading }\end{array}$} & 350 layer & 622.99 & 600.31 & 3.64 \\
\hline & 450 layer & 626.96 & 607.32 & 3.13 \\
\hline & 550 layer & 624.17 & 604.46 & 3.16 \\
\hline & 650 layer & 627.23 & 615.58 & 1.86 \\
\hline \multirow[t]{4}{*}{ The stage of failure } & 350 layer & 629.86 & 617.96 & 1.89 \\
\hline & 450 layer & 635.19 & 628.71 & 1.02 \\
\hline & 550 layer & 633.27 & 619.75 & 2.13 \\
\hline & 650 layer & 637.96 & 629.67 & 1.30 \\
\hline
\end{tabular}

Table 5.5 Theoretical calculation results of floor sandstone specimens are compared with experimental results

\begin{tabular}{|c|c|c|c|c|}
\hline The scanning stage & $\begin{array}{l}\text { Scanning } \\
\text { layer }\end{array}$ & $\begin{array}{l}\text { Experimental results } \\
\text { of CT Numbers }\end{array}$ & $\begin{array}{l}\text { Computed results } \\
\text { of CT Numbers }\end{array}$ & $\begin{array}{l}\text { Error } \\
(\%)\end{array}$ \\
\hline \multirow{4}{*}{$\begin{array}{l}\text { The stage of } \\
\text { unloading }\end{array}$} & 350 layer & 553.64 & & \\
\hline & 450 layer & 563.59 & & \\
\hline & 550 layer & 573.29 & & \\
\hline & 650 layer & 589.38 & & \\
\hline \multirow{4}{*}{$\begin{array}{l}\text { The end of the first } \\
\text { stage of loading }\end{array}$} & 350 layer & 583.53 & 538.84 & 7.66 \\
\hline & 450 layer & 579.37 & 544.99 & 5.93 \\
\hline & 550 layer & 573.68 & 548.55 & 4.38 \\
\hline & 650 layer & 567.98 & 551.96 & 2.82 \\
\hline \multirow{4}{*}{$\begin{array}{l}\text { The end of the second } \\
\text { stage of loading }\end{array}$} & 350 layer & 598.51 & 562.74 & 5.98 \\
\hline & 450 layer & 596.87 & 563.38 & 5.61 \\
\hline & 550 layer & 593.09 & 571.47 & 3.65 \\
\hline & 650 layer & 590.60 & 581.03 & 1.62 \\
\hline \multirow[t]{4}{*}{ The stage of failure } & 350 layer & 602.74 & 573.05 & 4.93 \\
\hline & 450 layer & 603.83 & 577.38 & 4.38 \\
\hline & 550 layer & 602.13 & 583.03 & 3.17 \\
\hline & 650 layer & 607.13 & 594.11 & 2.14 \\
\hline
\end{tabular}




\subsubsection{Construction of Crack Growth Factor Model}

A function or a curve of Logistic is a common form of $S$ function named after Pierre Francois Velure in 1844 or 1845 in order to reveal the relationship between the curve and population growth. The generalized curve of Logistic can be used to describe the shape of $S$ curve that represents the population growth $(P)$. The population grows exponentially at the initial stage; as the number grows, the population reaches saturation and the growth rate decreases. Finally, the growth rate decreases to zero when the population grows to the maximum allowable environment. As shown in Fig. 5.5, the function is expressed as follow: $\mathrm{y}=A_{2}+\frac{\left(A_{1}-A_{2}\right)}{\left(1+x / x_{0}\right)^{p}}$, where $x$ means any moment, $y$ represents the number of people corresponding to the time $x, A_{1}$ is the population at the initial moment, $A_{2}$ is the largest population that can be accommodated under natural and environmental conditions, $x_{0}$ represents the time corresponding to $A_{2} / 2, p$ is correlation coefficient.

Based on the Sect. 5.2.2, it showed that the chaotic characteristics of sandstone were chaotic under uniaxial cyclic load/unload. In this chapter, the cracks lengths and areas were calculated as the ordinate and the abscissa with the stress, the Logistic function was used to fit. Crack Growth Factor Model (CGFM) was proposed to describe the relationship between the length (and area) of cracks and stress. Fitting curve is $\mathrm{y}=A_{2}+\frac{\left(A_{1}-A_{2}\right)}{\left(1+x / x_{0}\right)^{p}}$, where each parameter in the equation is given practical meaning, the length or area of the cracks in any status is represented with the strain $y, A_{1}$ is used for the length or area of the cracks in the initial state, $A_{2}$ is used for the maximum length that the cracks can reach in the destructive status, Area value, $x_{0}$ is represented with corresponding to the stress value of $A_{2} / 2, p$ is power, as the correlation coefficient. So the original formula becomes $L=$ $L_{2}+\frac{\left(L_{1}-L_{2}\right)}{\left(1+\sigma / \sigma_{0}\right)^{P}}$ or $S=S_{2}+\frac{\left(S_{1}-S_{2}\right)}{\left(1+\sigma / \sigma_{0}\right)^{p}}$. The relationship between the crack lengthstress and crack area-stress curves was fitted under the cyclic load/unload conditions. The fitting parameters were shown in Figs. 5.6 and 5.7. The relevant parameters were shown in Tables 5.6 and 5.7.

Because of the different rock samples, the variation range of cracks length and area is different, that is to say, the S1, S2, L1 and L2 of each sample are different, and because $\sigma_{0}$ is related to the maximum value of length and area, the above

Fig. 5.5 Distribution of Logistic function

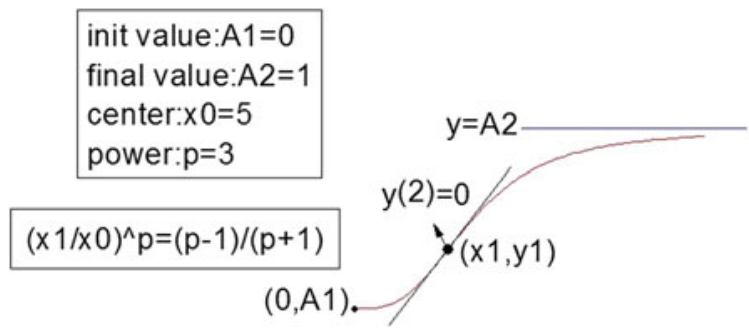




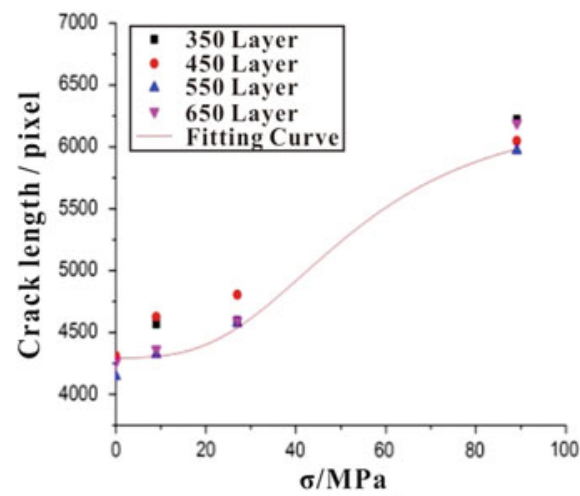

(a) Fitting curve of roof sandstone

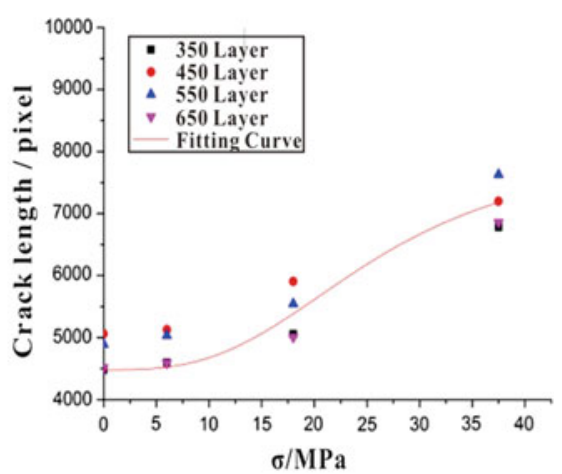

(b) Fitting curve of floor sandstone

Fig. 5.6 Fitting curves of cracks length-stress relation

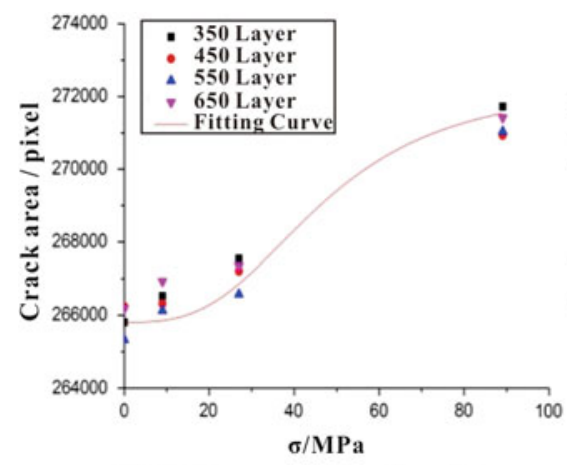

(a) Fitting curve of roof sandstone

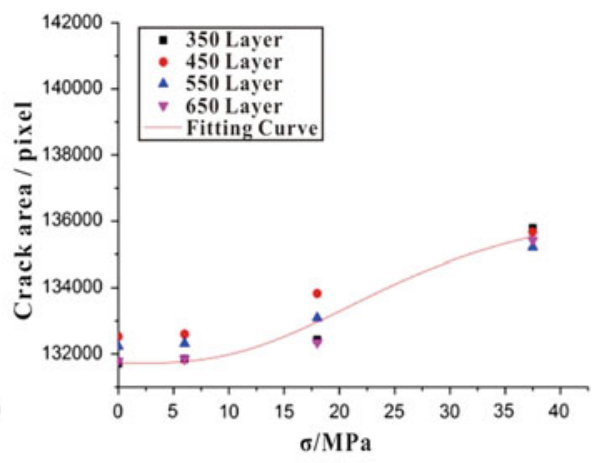

(b) Fitting curve of floor sandstone

Fig. 5.7 Fitting curves of cracks area-stress relation

Table 5.6 Related parameters and equations of fitting curves of crack lengths

\begin{tabular}{l|l|l|l|l|l}
\hline \multirow{2}{*}{ Crack length } & \multicolumn{4}{|l|}{ Related parameters } & Fitting equations \\
\cline { 2 - 5 } & $\mathrm{L}_{1}$ & $\mathrm{~L}_{2}$ & $\sigma_{0}$ & $\mathrm{p}$ & \\
\hline Roof sandstone & 4295.6 & 6330.9 & 52.66 & 3 & $L=6330.9+\frac{(4295.6-6330.9)}{(1+\sigma / 52.66)^{3}}$ \\
\hline Floor sandstone & 4480.8 & 8070.0 & 25.89 & 3 & $L=8070.0+\frac{(4480.8-8070.0)}{(1+\sigma / 25.89)^{3}}$ \\
\hline
\end{tabular}


Table 5.7 Related parameters and equations of fitting curves of crack areas

\begin{tabular}{l|l|l|l|l|l}
\hline \multirow{2}{*}{ Crack areas } & \multicolumn{4}{|l|}{ Related parameters } & Fitting equations \\
\cline { 2 - 6 } & $\mathrm{S}_{1}$ & $\mathrm{~S}_{2}$ & $\sigma_{0}$ & $\mathrm{p}$ & \\
\hline Roof sandstone & 265,810 & 272,380 & 46.95 & 3 & $S=272380+\frac{(265810-272380)}{(1+\sigma / 46.95)^{3}}$ \\
\hline Floor sandstone & 131,710 & 136,830 & 26.15 & 3 & $S=136830+\frac{(131710-136830)}{(1+\sigma / 26.15)^{3}}$ \\
\hline
\end{tabular}

expression is different. However, the evolution of cracks is consistent with the Logistic equation under uniaxial cyclic load/unload. Therefore, the length and area of cracks propagation process can be defined as the parameter $B$, so as to construct the CGFM based on the parameter $B$ :

$$
G=B_{2}+\frac{\left(B_{1}-B_{2}\right)}{\left(1+\sigma / \sigma_{0}\right)^{3}}
$$

where $G$ is growth factor of crack; $B_{1}$ is the initial value of crack parameter; $B_{2}$ is the destruction of the status of cracks can reach the maximum parameter value; $\sigma$ is the stress value; $\sigma_{0}$ is represented with corresponding to the stress value of $B_{2} / 2$; $p$ is the power, the correlation coefficient, CGFM of sandstone $\mathrm{p}=3$.

The model quantitatively describes the propagation and evolution of cracks in the process of sandstone fracture, which provides a theoretical basis for studying the effect of meso-structure change on the macroscopic damage of sandstone. In comparison of roof and floor sandstone, it can be inferred that other rock samples should be coincided with the model. Therefore, the model can be extended to all hard rock. However, there $p$ value of the size will change for different lithology of rock.

$$
G=B_{2}+\frac{\left(B_{1}-B_{2}\right)}{\left(1+\sigma / \sigma_{0}\right)^{\mathrm{p}}}
$$

where $G$ is growth factor of crack; $B_{1}$ is the initial value of crack parameter; $B_{2}$ is the destruction of crack status can reach the maximum parameter value; $\sigma$ is the stress value; $\sigma_{0}$ is represented with corresponding to the stress value of $B_{2} / 2 ; p$ is the power, the correlation coefficient, the selection of $p$ value is related to the lithology of rock sample.

It shows that CGFM is impossible to grow infinitely; the actual situation is that the crack grows to a certain number, and then the rate of crack growth will slow down. Because the objective cause of block effect have a certain impact on the crack growth, and the block effect will continue to increase with the growth of crack, the growth rate of crack is not a constant, it should be reduced. Finally, the crack grows to the maximum value when rock reach to the failure point, then the growth rate is reduced to 0 . In this chapter, the growth factor model of crack is a new attempt to describe growth process of rock crack and a new idea for predicting of rockburst. 


\subsection{Conclusion}

In this chapter, the fractal dimension, length and area of fractures in the whole process of sandstone under uniaxial cyclic load/unload were analyzed, and the quantitative description of CT test was completed, we obtained the following conclusions:

1. Under the cyclic load/unload, the internal cracks of sandstone specimens were gradually sprouted and expanded, which made the complexity of cracks structure increase and the fractal dimension increases. After the stress reached the peak, with the cracking of sandstone specimens, the degree of sample failure continued to increase. However, the shape and structure of cracks tended to be simplified, the complexity was reduced and the fractal dimension was reduced. Therefore, the fractal dimension could be used to quantitatively describe the evolution of cracks propagation in the process of rock failure.

2. The entropy model of rock system was constructed as $S=\mathrm{k} \times \ln \left(D_{0}+A \mathrm{e}^{-\frac{\left(\sigma-\sigma_{\mathrm{c}}\right)}{2 \times \omega}}\right)$. Under uniaxial cyclic load/unload, the fractal dimension change of cracks was combined with the evolution of quantitatively described system entropy. It was proved that the dimensionality reduction, entropy reduction and order were unified in the process of rock failure. The dynamic characteristics of rock system, which was from disorder to order, could be described by the process of dimension reduction and entropy reduction.

3. The evolution formula of CT number for sandstone under uniaxial cyclic load/ unload could be expressed by the equation after the corresponding transformation. Therefore, the evolution process of density of sandstone could be described by one-dimensional chaotic mapping Logistic equation under uniaxial cyclic load/ unload, and the evolution process of sandstone crack had chaotic characteristics.

4. In this chapter, CGFM was established with the equation $G=B_{2}+\frac{\left(B_{1}-B_{2}\right)}{\left(1+\sigma / \sigma_{0}\right)^{3}}$, which quantitatively described the evolution of crack propagation in the process of rock failure. When the cracks growed to a certain number, the growth rate of cracks increased with the blocking effect, then the rock was failure, the cracks growed to the maximum, and the crack growth rate became zero at this time.

\section{References}

Bobet A (2000) The initiation of secondary cracks in compression. Eng Fract Mech 66(2):187-219

Faloutsos C, Gaede V (1996) Analysis of n-dimensional quadtrees using the Hausdorff fractal dimension. Comput Sci Dep 548

Foroutan-pour K, Dutilleul P, Smith DL (1999) Advances in the implementation of the box-counting method of fractal dimension estimation. Appl Math Comput 105(2):195-210

Goldberger AL, Amaral LAN, Hausdorff JM et al (2002) Fractal dynamics in physiology: alterations with disease and aging. Proc Natl Acad Sci 99(suppl 1):2466-2472 
Heping X (1989) Studies on fractal models of the microfracture of marble. Kexue tongbao (English edition) 34(15):1292-1296

Hoxha D, Lespinasse M, Sausse J et al (2005) A microstructural study of natural and experimentally induced cracks in a granodiorite. Tectonophysics 395(1):99-112

Hudson JA, Priest SD (1979) Discontinuities and rock mass geometry. Int J Rock Mech Min Sci 16(6): 339-362

Li J, Du Q, Sun C (2009) An improved box-counting method for image fractal dimension estimation. Pattern Recogn 42(11):2460-2469

Lin P, Wong RHC, Chau KT et al (2000) Multi-crack coalescence in rock-like material under uniaxial and biaxial loading. Key Eng Mater 183:809-814

Mandelbrot BB (1977) Fractals. Wiley, New York

Mandelbrot BB (1999) Fractal object: the shape, the opportunities and dimension. World Publishing Corporation, Beijing

Mandelbrot BB, Pignoni R (1983) The fractal geometry of nature. WH freeman, New York

Nolen-Hoeksema RC, Gordon RB (1987) Optical detection of crack patterns in the opening-mode fracture of marble. Int J Rock Mech Min Sci 24(2):135-144

Priest SD, Hudson JA (1976) Discontinuity spacings in rock. Int J Rock Mech Min Sci 13(5):135148

Reyes O, Einstein HH (1991) Failure mechanisms of fractured rock-a fracture coalescence model. Int Soc Rock Mech 10(1):333-340

Shen B, Barton N (1997) The disturbed zone around tunnels in jointed rock masses. Int J Rock Mech Min Sci 34(1):117-125

Shen B, Stephansson O, Einstein HH et al (1995) Coalescence of fractures under shear stresses in experiments. J Geophys Res 100(6):5975-5990

Wong RHC, Chau KT, Tang CA et al (2001) Analysis of crack coalescence in rock-like materials containing three flaws - part I: experimental approach. Int J Rock Mech Min Sci 38(7):909924

Wong TF (1982) Micromechanics of faulting in Westerly granite. Int J Rock Mech Min Sci 19 (2):49-64

Wong TF, Wong RHC, Jiao MR et al (2004) Micromechanics and rock failure process analysis. Key Eng Mater 261(263): 1

Xie Heping, Chen Zhida, (1988) Fractal geometry and fracture of rock. Acta Mechanica Sinica 4 (3):255-264

Open Access This chapter is licensed under the terms of the Creative Commons Attribution-NonCommercial 4.0 International License (http://creativecommons.org/licenses/by-nc/ 4.0/), which permits any noncommercial use, sharing, adaptation, distribution and reproduction in any medium or format, as long as you give appropriate credit to the original author(s) and the source, provide a link to the Creative Commons license and indicate if changes were made.

The images or other third party material in this book are included in the book's Creative Commons license, unless indicated otherwise in a credit line to the material. If material is not included in the book's Creative Commons license and your intended use is not permitted by statutory regulation or exceeds the permitted use, you will need to obtain permission directly from the copyright holder. 


\section{Chapter 6 \\ Experimental Investigation on AE \\ Precursor Information of Rockburst}

\subsection{Introduction}

Macro-fracture of rockmass is caused by nucleation, extension and propagation from micro-cracks among the meso-structures. So, it is important to obtain the evolution pattern from micro-facture to meso-scale. Rock fracture is accompanied with crystal dislocation, crystals slip, elastoplastic deformation, crack initiation, and propagation. Meanwhile, energy is released in the form of stress waves which can be monitored. Stress wave monitoring techniques, such as AE and micro-seismic (MS) monitoring, have been used for many decades to study the fracturing behavior of rock. As an important method of dynamic hazard monitoring, it can obtain the intensity and frequency of microseismic events activity and the location distribution of microseismic events, so we can estimate the potential dynamic hazard and obtain the precursory information to achieve the predicting hazards.

However, it is difficult to achieve the field practice from the rock experiment. Meanwhile, the lab test is an effective method to research the rock failure. Therefore, it's the primary task to analyze the changing characteristics and the precursory information pattern of rockburst.

In recent decades, many scholars obtained some relevant research in precursory information characteristics of rockburst. Under uniaxial compression test, the appeared relatively quiet period of $\mathrm{AE}$ was found before the rock failure, and this phenomenon was used as precursory information of rockburst (Wang 2014). Carpinteri et al. (2007) found that the rate of AE energy was accelerating before the sample failure. Lacidogna et al. (2011) found that AE signal was consistent with damage and evolution process of brittle rock, and taken the action that the stress was deeply down as failure precursor. Wang et al. (2015a, b) found relative quiet period before the rock failure, the predicting point of rockburst was proposed and some correlative theories were obtained in field practice. Meng et al. (2016) analyzed the energy evolution feature of red sandstone deformation under the different cycle loading rates. Furthermore, the implied conversion pattern between energy 
with dissipation, Carpinteri and Lacidogna (2006) analyzed evolving relation between AE energy and dissipation energy under the concrete beam loading using the three point bending test.

In a word, many scholars conducted lots of AE experiments; most of their work was focused on the relationship between AE parameters with rock fracture and failure. However, in this chapter, the precursor of rock failure was utilized to predict rockburst using the distribution feature and evolution pattern of AE/MS in the deep mining.

\subsection{Spatio-temporal Evolution Pattern of Rock Failure}

Under the external loading, the virgin rock cracks were initiated, propagated, and linked. At the same time, AE events were released in the form of elastic stress waves. Therefore, with the spatial-temporal distribution characteristics of $\mathrm{AE}$ source 3D-location, the characteristics of rock cracks initiating, propagating and linking was analyzed, AE evolution regular and the precursor feature of limestone failure were revealed finally.

\subsubsection{AE Experiment}

In this experiment, 10 limestone specimens were divided into two groups. During the testing, the axial equivalent displacement loading method was used to load the specimens, and AE monitoring system was synchronously operated. The loading speed was controlled at $0.5 \mathrm{~mm} / \mathrm{min}$. In the AE monitoring testing, data collection was performed six channels, and the sampling frequency was set at $1 \mathrm{MHz}$. The threshold voltage value was set at $100 \mathrm{mv}$ and the threshold amplitude value was set at $45 \mathrm{~dB}$. AE signals would be filtered for removing noise signals, and a high signal/noise ratio could be achieved. During the test, six nano30 sensors were used to monitor AE signals and the pre-amplifier gain was set at $40 \mathrm{~dB}$. The sensors were fixed on rock surface by adhesive band and petroleum jelly. In order to reduce the influence of the end effect, there should be $20 \mathrm{~mm}$ distances between the end of samples and the sensors. The layout of AE sensors were illustrated in Fig. 6.1.

\subsubsection{Principle of AE Event Location}

In order to locate the micro-cracks inside rock sample by $\mathrm{AE}$ monitoring, the sensors were arranged in a geometric pattern to form the sensor array on the surface of rock sample. The location of AE source was then determined based on AE signal parameters and location methods. 
Fig. 6.1 The arrangement of AE sensors (Unit mm)

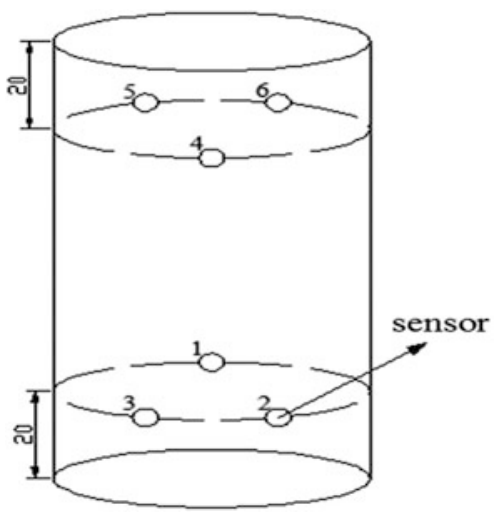

For the sudden type of AE signals, time difference location method and regional location method were mainly used to achieve the goal. Combined with the spatial location of sensor, $\mathrm{P}$ wave velocity and other related parameters, the former method was usually use as the measurement of parameters, such as the time difference among AE channel signals reaching the sensors to determine the location of $\mathrm{AE}$ source by the simplex algorithm or the Geiger algorithm. This location method was widely used in small rock samples and large components because of its higher accuracy, though the calculation was more complex than the latter one. Due to the simpler location operation and higher processing speed, the regional location method was generally applicable to situations where the location accuracy was low and the time difference could not be located.

The simple location algorithm and Geiger location algorithm (Miller et al. 1999) were two commonly used algorithms of AE location. The principle of these two algorithms was to invert the location of $\mathrm{AE}$ source by picking up the P-wave arrival time difference through different position sensors. Geiger algorithm was one of Gauss-Newton minimum fitting function applications, which was suitable for AE/ MS and earthquake events in small areas. In this chapter, Geiger algorithm was used to locate the AE source based on the time difference between the arrivals of $\mathrm{P}$ wave. In order to determine the real AE source coordinates, this algorithm was done by iterating over a hypothetical AE source. In each iteration process, a correction vector $\Delta \theta$ was calculated by least squares method, and added it to the previous iteration result to obtain a new $\mathrm{AE}$ source location coordinate, then put the new coordinate parameters into the time-distance equation (6.1) to judge whether the new coordinate satisfies the equation. If the requirement was met, the new coordinate was the location of AE source; otherwise, it was need to continue iterating until the time distance equation was satisfied. In the AE location test, sensors were arranged in accordance with Fig. 9.3, and the location of $\mathrm{AE}$ events could be determined by measuring the relative time difference between each sensor picking up the $\mathrm{P}$ wave. 
The time-distance discriminant equation for the iteration results is as follows:

$$
\left(x_{i}-x\right)^{2}+\left(y_{i}-y\right)^{2}+\left(z_{i}-z\right)^{2}=\left[v_{p}\left(t_{i}-t\right)\right]^{2}
$$

where $x, y, z$ are the initial assumed AE source coordinates; $t$ is the time at which the initial $\mathrm{AE}$ event occurs; $x_{i}, y_{i}, z_{i}$ are the spatial coordinates of $i$ th sensor, respectively; $v_{p}$ is the velocity of $\mathrm{P}$ wave; $t_{i}$ is the time that the $i$ th sensor picks up the $\mathrm{P}$ wave.

The time at which the $\mathrm{P}$ wave propagates to the $i$ th sensor is $t_{0, i}$, The first-order Taylor expansion of $\mathrm{P}$ wave arrival time can be calculated using the initial assumed AE source coordinates:

$$
t_{0, i}=t_{c, i}+\frac{\partial t_{i}}{\partial x} \Delta x+\frac{\partial t_{i}}{\partial y} \Delta y+\frac{\partial t_{i}}{\partial z} \Delta z+\frac{\partial t_{i}}{\partial t} \Delta t
$$

where,

$$
\begin{gathered}
\frac{\partial t_{i}}{\partial x}=\frac{x_{i}-x}{v_{p} R}, \quad \frac{\partial t_{i}}{\partial y}=\frac{y_{i}-y}{v_{p} R}, \quad \frac{\partial t_{i}}{\partial z}=\frac{z_{i}-z}{v_{p} R}, \quad \frac{\partial t_{i}}{\partial t}=1, \\
R=\sqrt{\left(x_{i}-x\right)^{2}+\left(y_{i}-y\right)^{2}+\left(z_{i}-z\right)^{2}}
\end{gathered}
$$

where $t_{c, i}$ is the time that the $\mathrm{P}$ wave spread from the assumed AE point coordinates to the $i$ th sensor. There were six sensors in this experiment for three-dimensional location. Therefore, six equations were listed in the form of a matrix as follow:

$$
\left[\begin{array}{cccc}
\frac{\partial t_{1}}{\partial x} & \frac{\partial t_{1}}{\partial y} & \frac{\partial t_{1}}{\partial z} & 1 \\
\frac{\partial t_{2}}{\partial x} & \frac{\partial t_{2}}{\partial y} & \frac{\partial t_{2}}{\partial z} & 1 \\
\vdots & \vdots & \vdots & \vdots \\
\frac{\partial t_{6}}{\partial x} & \frac{\partial t_{6}}{\partial y} & \frac{\partial t_{6}}{\partial z} & 1
\end{array}\right]\left(\begin{array}{c}
\Delta x \\
\Delta y \\
\Delta z \\
\Delta t
\end{array}\right)=\left(\begin{array}{c}
t_{0,1}-t_{c, 1} \\
t_{0,2}-t_{c, 2} \\
\vdots \\
t_{0,6}-t_{c, 6}
\end{array}\right)
$$

The modified vector $\Delta \theta$ in Eq. (6.3) was solved by Gauss elimination method, which was $\Delta \theta=[\Delta x, \Delta y, \Delta z, \Delta t]$. After the modified vector $\Delta \theta$ was calculated for each possible $\mathrm{AE}$ source coordinate matrix, the iteration was continued with $(\theta+\Delta \theta)$ as the new coordinates until the Eq. (6.1) was satisfied. At this time, the satisfied coordinates could be used as the real location. 


\subsubsection{Spatio-temporal Distribution Characteristics of Rock Failure}

As the primary micro-cracks closed, expanded and linked, each sample generates lots of $\mathrm{AE}$ events in the entire loading process. The collecting system could be manipulated the $\mathrm{AE}$ date and obtained the visualized location images. Take only X2 sample date for example, as shown in Figs. 6.2 and 6.3.

As shown in Fig. 6.2, when the stress was at low level $\left(<0.2 \sigma_{c}\right)$, AE activity was active and gathered round middle part and top part of sample. This phenomenon could be explained as follows: in one hand, the original developmental cracks of samples were gradually closed under compression loading; on the other hand, a small quantity of $\mathrm{AE}$ events were occurred because of friction between samples and the pressure plate of machine. In the elastic deformation and stable growth crack stage $\left(0.4-0.6 \sigma_{c}\right)$, AE counts increased steadily and accompanied with the development of microfracture. AE events dispersed gradually from the middle part to around part. When stress increased at the $0.8 \sigma_{c}$, AE events increased quickly and gathered round the main fracture, which presented obvious zonal distribution. The sample surface began showing the macrocrack, and AE event increased. In the post-failure stage, AE activities did not stop, but stress dipped quickly.

As the spatial distribution of AE events change with the loading time, we have analyzed the feature of spatio-temporal evolution in the different loading stage.

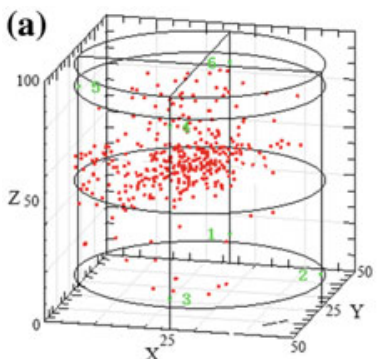

(d)

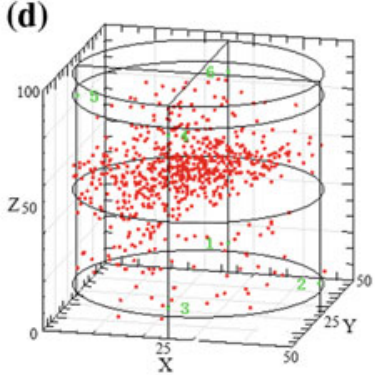

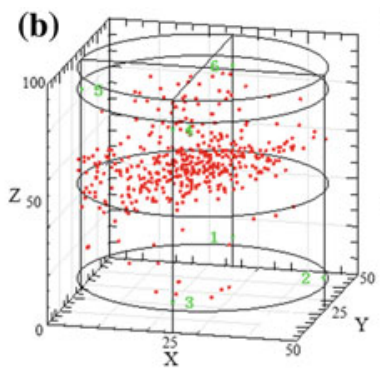

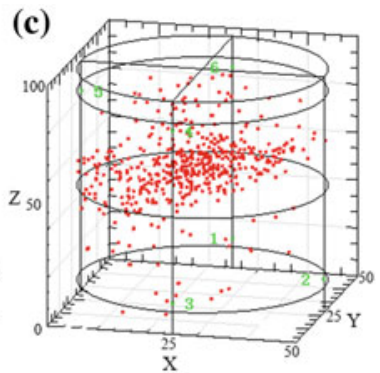

(e)

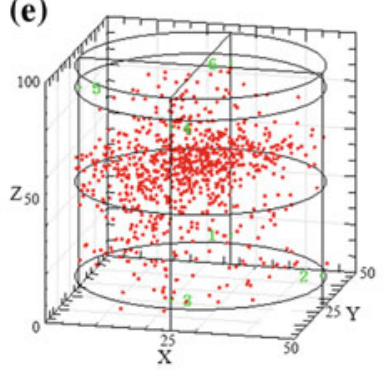

Fig. 6.2 AE distribution of $\mathrm{X} 2$ sample during the different loading period 

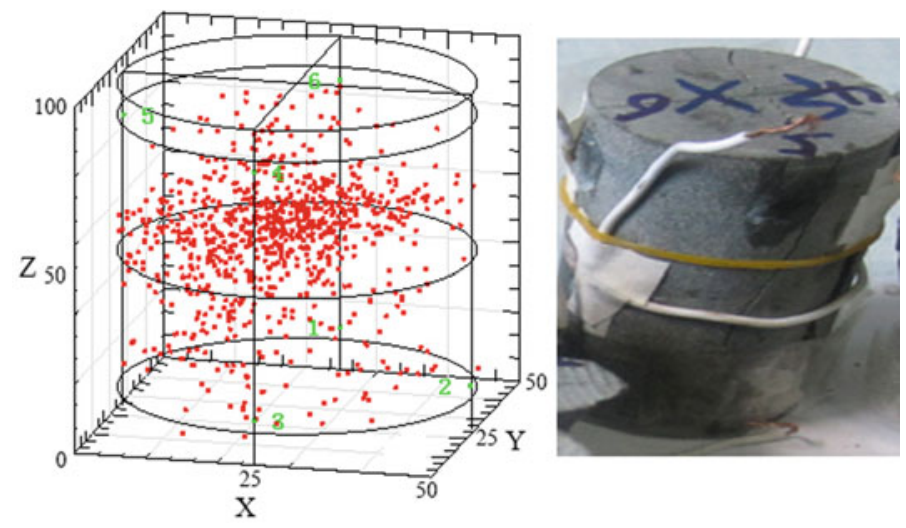

Fig. 6.3 Comparison of AE localization and actual rupture propagation

Though the distribution was very heterogeneous, we still found two main phenomenons after summarizing all sample results, as shown in Figs. 6.2 and 6.3:

(1) Intensive phenomenon: It was easy to be found that AE events occurred mainly in the middle part of X2 sample. However, when the stress increased to the 40, 60 and $80 \%$ of peak stress, AE events were always around the main plane of fracture, and only few events was in others parts. Therefore, it was an effective way to predict roughly the final position and attitude of main fracture plane using the spatial distribution of AE event.

(2) Zonation: AE events internal sample presented the obvious zonate shape, and the location of shape was basically consistent with the final macro-fracture. The main fracturepart from the zonation was shown.

\subsection{Characteristics of Relatively Quiet Period for Rock Failure}

\subsubsection{Deformation and Failure Characteristics of Loaded Rock}

Stress-strain curves of limestone samples were shown in Fig. 6.4, the deformation trend of a specimens were similar during the entire loading process. There are four basic stages of variation trend, including the original crack compaction, the stable crack growth, the unstable crack propagation and the rock failure stage (Wang et al. $2015 a, b)$. In the chapter, only one sample was choosed to analyze and discuss the mechanical properties and AE characteristics of the loaded rock. As shown in Fig. 6.6, the sample No. 3 was appeared the original crack compaction stage after 


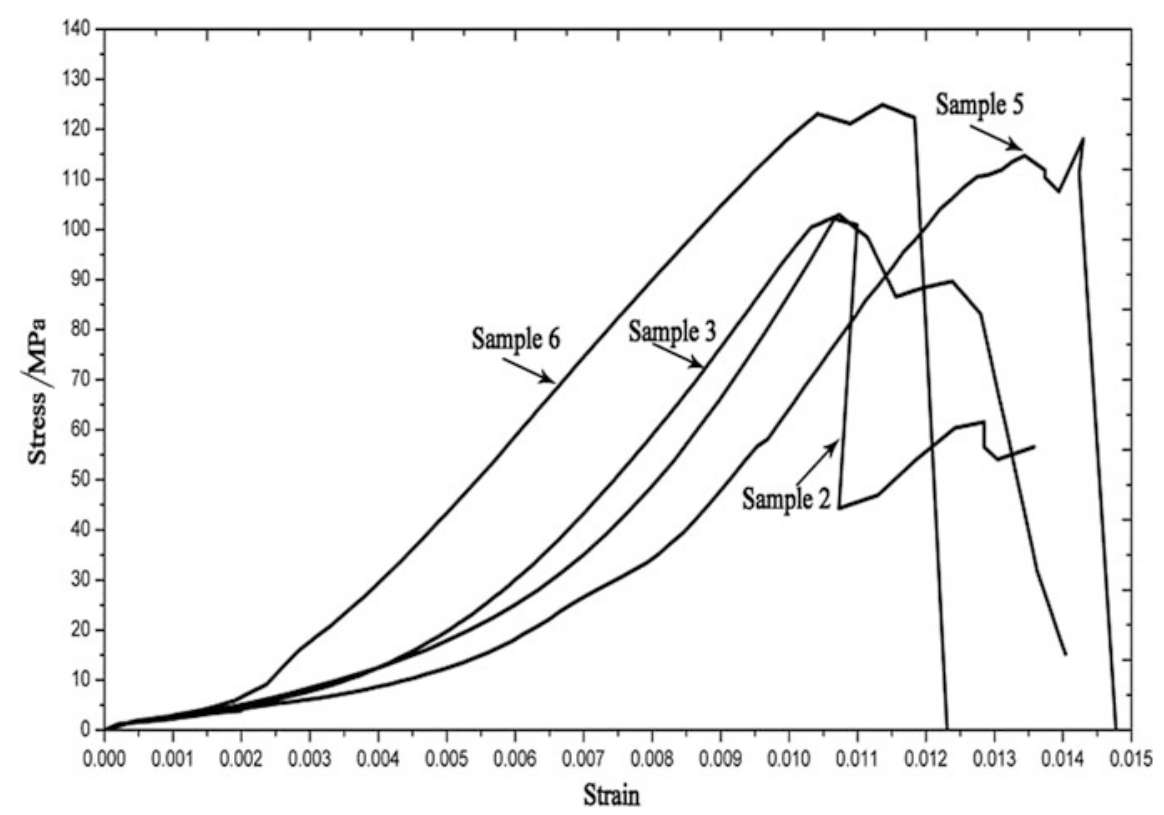

Fig. 6.4 Stress-strain curves of samples. With kind permission from "AMER SOC TESTING MATERIALS", Wang et al. (2015b), 1296-1307 Fig. 6, all rights reserved

the beginning of uniaxial compression. With the stress increasing, the sample came into elastic deformation and the curve remained linear variation because of stable crack growth, it spended for a long time. Cracks initiated, extended and developed into the unstable propagation. In the plastic deformation stage, curve started to change non-linearly and rock volume also increased slightly. It was thought that many micro-fractures propagated and jointed one another which caused the volume increase of mesostructures. When the microcracks jointed and formed into macroscopic cracks, rock relative stability was destroyed, and stress reached the peak 102.89 MPa (Wang et al. 2015a, b). Stress didn't dipped sharply after the peak, and rock still had certain supporting ability. Therefore, there was secondary stress drop in the post-failure stage. Due to heterogeneity of rock, there was different destruction style, such as oblique shear failure and tensile failure. In the same way, the different mechanical properties of samples was because the size and the heterogeneity of rockmass. The analyzation of rock mechanical properties was important to understand the AE phenomenon (Wang et al. 2015a, b). 


\subsubsection{Relationship Between the Constitutive Characteristics and the Accumulative AE Counts}

We could clearly find the relationships of sample No. 3 between stress-strain curve and the accumulative AE counts in Fig. 6.5. AE activity was constantly changed during the whole uniaxial loading (Wang et al. 2015a, b). However, there were still several changing characteristics in the accumulative AE counts. Before stress reached $20 \%$ of the peak stress, the original cracks were gradually closed and the friction occurred between samples and the pressure plate which generated a quantity of AE events occurred, the slope of this stage was larger than the slope of the elastic stage in the accumulative count curve. In the elastic deformation stage, cracks were in stable growth, and AE activity appeared obviously changing trend at the $30 \%$ of the peak stress, which we could regard as the Kaiser Effect point. From 30 to $60 \%$ of the peak stress, AE accumulative counts were linearly increased, which meanted AE activity stably developing. From Fig. 6.5, it was easy to discover that AE activity was unstable by comparison, which laterally reflected the heterogeneity of rock properties. When stress reached $60 \%$ of the peak stress, rock came into the plastic deformation stage and $\mathrm{AE}$ activities were enhanced. When stress was approximately at $75 \%$ of the peak stress, there were almost no AE activity and this phenomenon lasted $5 \mathrm{~s}$. In other words, the sample deformation was in a relatively

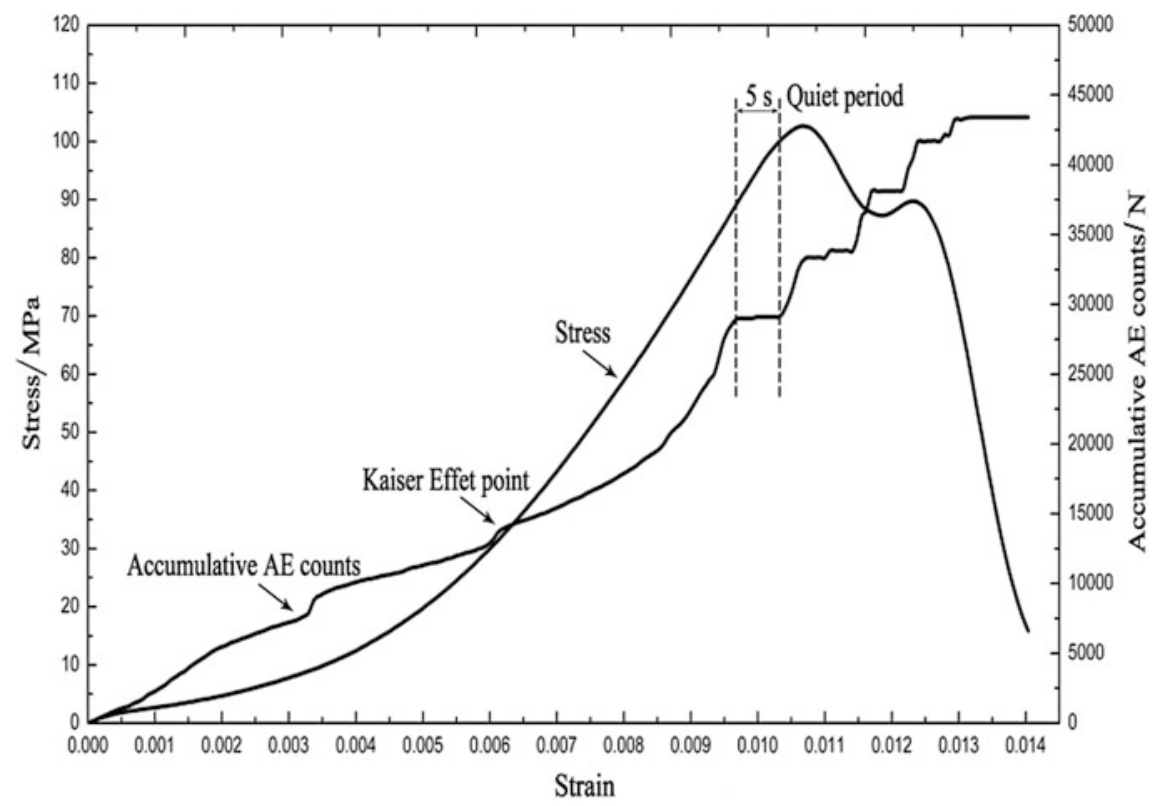

Fig. 6.5 Relationship between stress-strain curves and accumulative AE counts for limestone sample. With kind permission from "AMER SOC TESTING MATERIALS", Wang et al. (2015b), 1296-1307 Fig. 7, all rights reserved 
quiet period as marked in Fig. 6.5. Then AE activity generated actively. When stress passed through the yield-point to peak stress, AE activities were rapidly enhanced, and the parameters of AE events were also increased, such as the amplitude, energy, frequency, duration, et al, which implied the magnitude of AE event stronger, and the size of fracture bigger. Therefore, in author's opinion, we could use the relatively quiet period as the precursor of rock failure to provide a predicting signal for rockmass instability. Similarly, we could use this opinion to analyze the micro-seismic events of seismicity to evaluate the rockmass stability in mining engineering (Wang et al. 2015a, b). After the peak stress, rock damage was in the post-failure, but AE activities did not stop, and intermittent emission constantly appeared. With stress declining, AE count increased periodically.

\subsubsection{Relationship Between AE Rate and Constitutive Characteristics}

Due to the samples inhomogeneous, mechanical and AE characteristics are nonlinear under uniaxial compression. Meanwhile, AE rate represents the intensity of AE activity and the developing speed of rock microcrack. Therefore, AE parameters of time domain can clearly reflect the real-time damage evolution, such as AE rate, $\mathrm{AE}$ energy rate and $\mathrm{AE}$ amplitude. $\mathrm{AE}$ duration and $\mathrm{AE}$ frequency reflect the properties of single $\mathrm{AE}$ event. In order to legibly understand the characteristics of $\mathrm{AE}$ and find the feature of $\mathrm{AE}$ relatively quiet period, we need to analyze $\mathrm{AE}$ parameters respectively.

$\mathrm{AE}$ rate is a sensitive parameter about real time, which can reflect the frequency of $\mathrm{AE}$ activities and evaluate the damage status. The relationships between the constitutive characteristics and AE rate about the sample NO. 3 are shown in Fig. 6.6.

From the above, it was easy to discover that $\mathrm{AE}$ activity was always active during the whole uniaxial compression. As shown in Fig. 6.6, AE accumulative count appeared a small increase at $15 \%$ of the peak stress. We also found AE rate sharply increased to 800 at $25 \mathrm{~s}$ which was caused by inhomogeneous development of microcrack in the sample. In the initial deformation stage, AE rate increased firstly which implied the initial microcavities closed gradually. After $10 \mathrm{~s}$, the rate became dipping trend and appeared a sharp increase around the time $25 \mathrm{~s}$. Then AE activity maintained steady change in the elastic rock deformation, and the rate also appeared an obvious increase at the Kaiser Effect point. In the plastic deformation damage, the rate increased gradually and stress appeared the linear increase. In this stage, cracks propagation was unstable, and micro-cracks merged progressively. Before $70 \mathrm{~s}$, the rate appeared a sharp increase, but stress curve didn't appear any dip trend. However, AE rate decreased sharply afterwards and there was a relatively quiet period appeared and lasted $5 \mathrm{~s}$ before the stress reached the peak stress. The loaded sample quickly passed through the quiet period before the arrival of the peak 


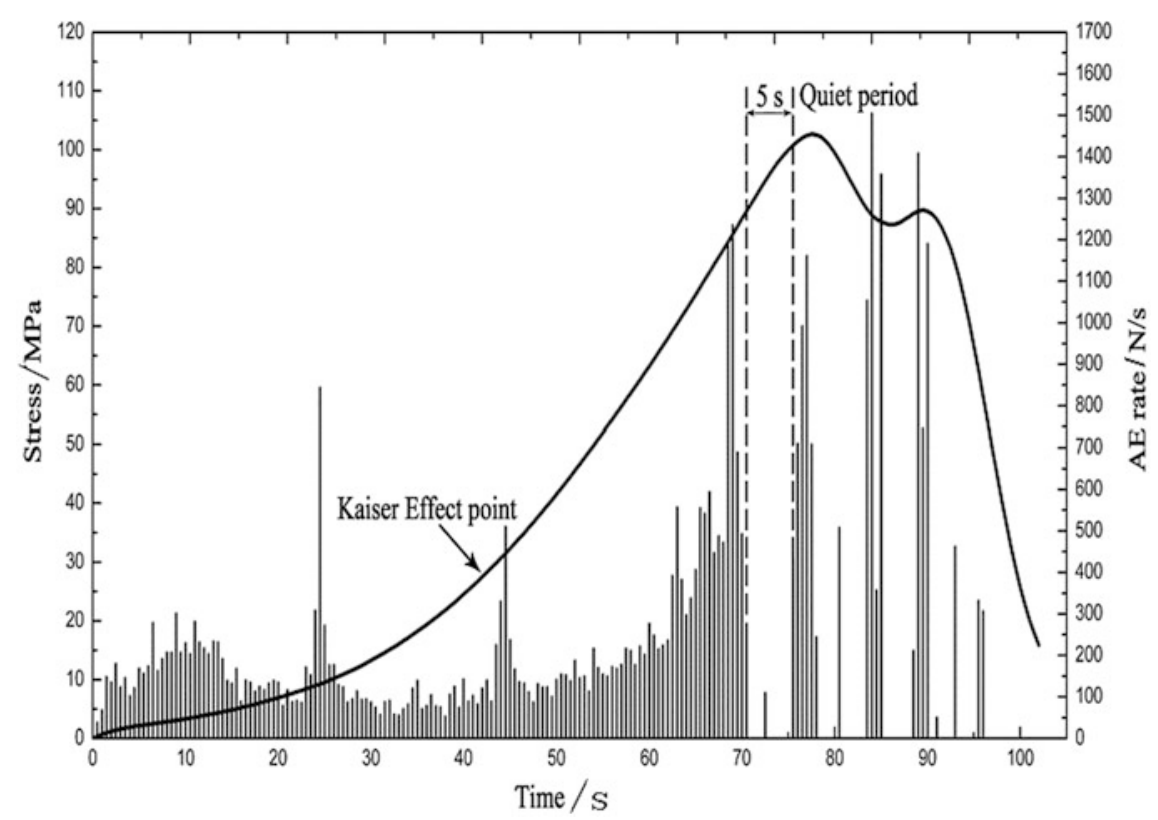

Fig. 6.6 Relationship between stress-time curves and AE rate for limestone sample. With kind permission from "AMER SOC TESTING MATERIALS", Wang et al. (2015b), 1296-1307 Fig. 8, all rights reserved

stress. AE rate increased rapidly when stress reached the peak. In the post-failure damage, $\mathrm{AE}$ rate became intermittent and several periods appeared with the process of stress decline. From the change trend of AE activity in the failure stage, we could easily find how strong the intensity of rock damage (Wang et al. 2015a, b).

\subsubsection{Evolution Characteristics of AE Amplitude in the Time Domain}

Figure 6.7 was a scatter diagram in which each scatter point represents a single AE event. AE amplitude was the maximum amplitude value of each $\mathrm{AE}$ wave which could be gained by Fourier Transform, which reflected the magnitude and intensity of $\mathrm{AE}$ activity. In view of the effectivity of $\mathrm{AE}$ extraction and the environment noise, we set the threshold at $45 \mathrm{~dB}$. Therefore, the minimum amplitude of $\mathrm{AE}$ signals was $45 \mathrm{~dB}$. As shown in Fig. 6.7, most amplitude of AE signals was below $60 \mathrm{~dB}$, and few of $\mathrm{AE}$ signals were beyond $80 \mathrm{~dB}$. As the amplitude reflected $\mathrm{AE}$ event energy directly, majority of AE events were had low-energy. On the contrary, only $0.7 \%$ of the total events were high energy. In other words, local slight fractures generated in the samples during the entire loading process. In the initial loading 


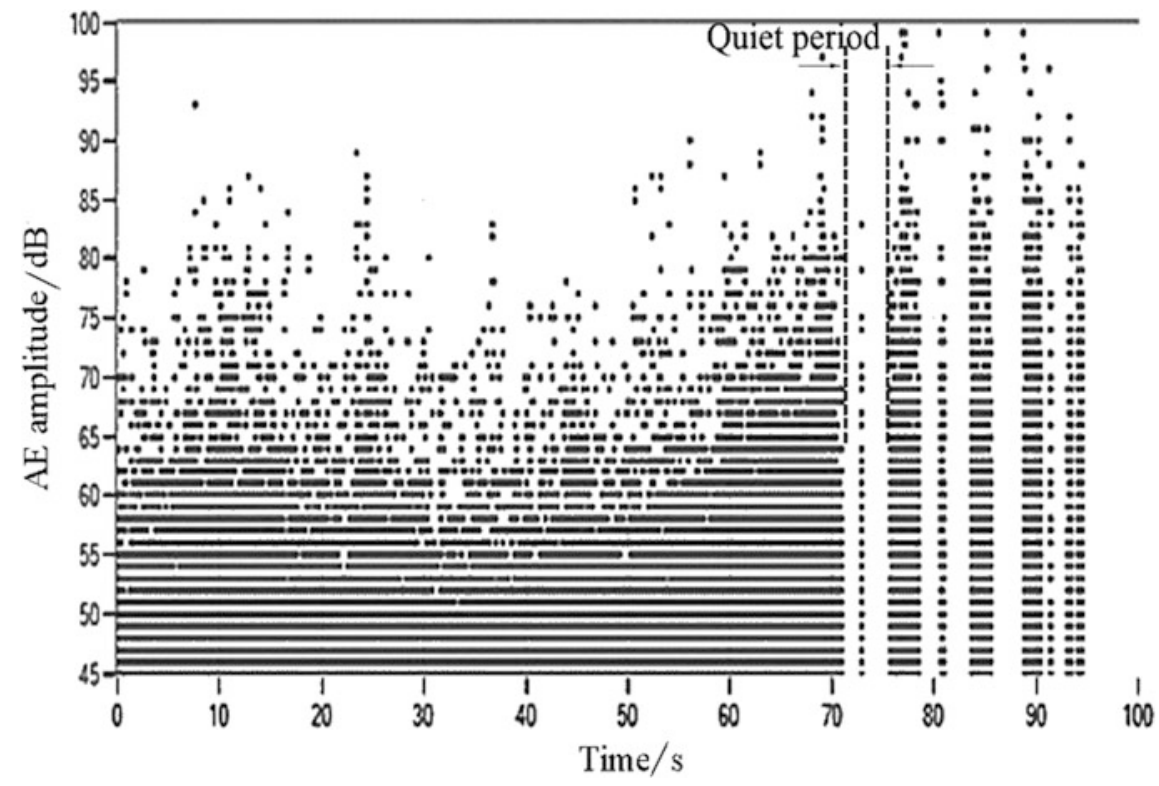

Fig. 6.7 Relationship between $\mathrm{AE}$ amplitude and time for limestone sample. With kind permission from "AMER SOC TESTING MATERIALS", Wang et al. (2015b), 1296-1307 Fig. 10, all rights reserved

stage, most of low-energy AE events were generated, and some of amplitudes began distributing beyond $80 \mathrm{~dB}$, which represented the completion of carities closing and the beginning of microcracks propagation. In the elastic deformation, the AE amplitudes were steady distributed in the time domain. But in the plastic deformation stage, the amplitudes presented the ascent distribution. Then in the unstable crack propagation stage, lots of high-amplitude AE signals appeared rapidly. When stress reached $75 \%$ of the peak stress, a relatively quiet period appeared as shown in Fig. 6.7. After the peak stress, rock strain began softening, and many high-amplitude and high-energy AE events were generated in the same time, which reflected the high energy was releasing with rock failure instability (Wang et al. 2015a, b).

\subsubsection{Analyzation of Precursory Characteristics of Rock Failure}

During the testing, a relatively quiet period of $\mathrm{AE}$ generation was found before rock failure during the test. To some extent, AE activities implied stress state of micro-fracture and reflected the damage evolution in the rock. But there must be some factors which caused the AE intermittent existed in the whole compress process. 
On the one hand, with uniaxial compressive stress increasing, strain energy of rock system transformed as deformation energy and dissipated energy. In the different deformation stages, energy percent of deformation and dissipation was relatively changing, which could be due to rock inhomogeneity, deformation energy could be also divided into volume energy and fracture energy, as the dissipated energy could be divided into the thermal energy, AE energy and radiant energy. The dissipated energy could be regarded as energy release with system energy import. Therefore, there was a balanced time domain in the process of energy import and energy release, which caused the propagation of micro-cracks, and energy release reach dynamic and temporary balance before the peak stress. This is one of reasons we can see the relatively quiet period in the loaded time domain.

On the other hand, high-damage intensity caused the sharp attenuation of $\mathrm{AE}$ propagation before the peak stress. There were many microstructural cracks existed in sample, which caused inhomogeneity of rock properties. Therefore, the propagation of $\mathrm{AE}$ waves was badly effected and the quiet period appeared. With stress increased, the state of balance was broken, and macro-fracture was formed causing the stress dip. The relatively quiet period might be mainly caused by inhomogeneity of samples (Wang et al. 2015a, b).

Therefore, we can use the AE quiet period as precursory information for prediction of rock failure and the practical engineering applications.

\subsection{Variation Regulation of AE Energy Parameters for Rock Failure}

\subsubsection{Experimental Setup and Procedure}

In the experiment, 10 granite specimens were tested, approximately $50 \mathrm{~mm}$ in diameter and $100 \mathrm{~mm}$ in height. The loading machine and $\mathrm{AE}$ testing system were operated at the same time to obtain the mechanical behaviors and AE dates of specimens under uniaxial compression. The loading speed was $0.005 \mathrm{~mm} \times \mathrm{s}^{-1}$. In addition, AE sampling frequency was set at $1 \mathrm{MHz}$. Six AE sensors were pasted on the surface of sample to detect $\mathrm{AE}$ waves. The pre-amplifiers were set at $40 \mathrm{~dB}$. Moreover, eliminating the influence of background noise, the threshold value of PCI-II acquisition system was set at $45 \mathrm{~dB}$.

\subsubsection{Energy Parameters Variations in Each Channel}

As six sensors were installed on the sample surface, it was meant that there were six channels could detect the same AE waves. Due to the inhomogeneity of rock properties, the attenuation of $\mathrm{AE}$ signal might be different in local positions. 
Fig. 6.8 AE energy parameter variations for each channel. From Wang et al. (2015a, b)

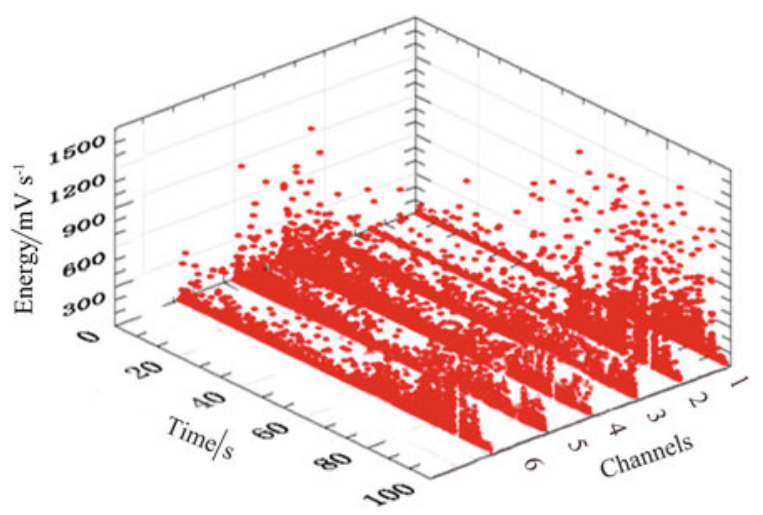

Therefore, one AE signal might be detected by the different position sensors, and some of sensors might not receive the signal, which caused AE energy parameter variations were different for each channel. Variation trend of AE energy was shown in Fig. 6.8, energy of 6 channels almost had the same variation trend. In the initial loading stage, AE energy increased firstly and then decreased subsequently. Energy of channel 1 and channel 2 was relatively insensitive, because the attenuation of AE energy was seriously when wave reached at the sensors. During the medium stage, AE energy kept plain evolution. After $70 \mathrm{~s}$, AE energy increased sharply and reached at the peak, which lasted in the almost the entire failure process. With the destruction of rock supporting stability, AE energy also sharply dipped. The reasons for this phenomenon could be explained as follow. The original inner cavities were compacted with lots of energy releasing. While in the elastic stage, rock deformation and cracks propagation were generally stable. Thus, AE energy release was also stable. However, as rock in the plastic stage, micro-fractures were in unstable expansion. Then, microcracks jointed macrocrack which led to rock failure while high energy fiercely released (Wang et al. 2015a, b).

\subsubsection{Relationship of Energy and Stress-Strain Curve on Time Domain}

As shown in Fig. 6.9, there was a fine relation between AE energy release and constitutive characteristics of loaded rock. It was obvious to find that most of $\mathrm{AE}$ signal energy was $300 \mathrm{MV} / \mathrm{s}$, and high energy event appeared in high stress stage, which directly reflected the stability of damage evolution. AE energy had different stages of relevant changing characteristics, describing as follow: the original crack compaction stage, the elastic deformation stage, the plastic deformation stage and the failure stage. During the first stage and the second stage, rock got the compression, but rock kept strong elastic property, so stress can be linearly developing. In this stage, a lot of $\mathrm{AE}$ waves had been generated with the cavities compaction 
and cracks initiation. In the third stage, rock sample was in plastic deformation and cracks developing were relatively stable. Before the yield stress, there was relatively stable AE energy release which reflected the balance of energy transformation. After $65 \mathrm{~s}$, stress curve bagan changing in the unstable slope, directly reflecting the strain unstability. Meanwhile, many high energy signals were generated and implied the intense cracks expansion (Wang et al. 2015a, b). Therefore, variation characteristics could be applied to replace the field stress monitoring as the evaluation factors of rockmass stability. As AE signal energy increased abruptly, it could be thought as the precursor for rock rupture.

\subsubsection{Analysis of Damage Features}

As shown in Fig. 6.9, high AE energy began appearing after $65 \mathrm{~s}$, which meant high-intensity damage in the sample. Therefore, it was very important to analyze the energy evaluating characteristics of this period. In this chapter, we selected the summation energy, average energy and maximum energy to analyze as shown in Figs. 6.10, 6.11, and 6.12. Three kinds of energy are defined as follow:

Summation of energy was the cumulative value of AE energy detected in all channels at the same time; the average of energy was the value as the total energy divided by the number of channels at a certain time; the maximum of energy was the maximum energy among each channel detected at a certain time. As the above, there should be pointed out that a channel could detect multiple energy signals in the same time, but each channel could not detect one event energy at the same time, which caused three values different at the same time point (Wang et al. 2015a, b).

As shown in Figs. 6.10, 6.11, and 6.12, three initial indexes were presented the same variation trend from 69 to $99 \mathrm{~s}$, but the values jumped in the time domain. So we had fitted the initial curve plotted in Figs. 6.10, 6.11, and 6.12. From the fitted

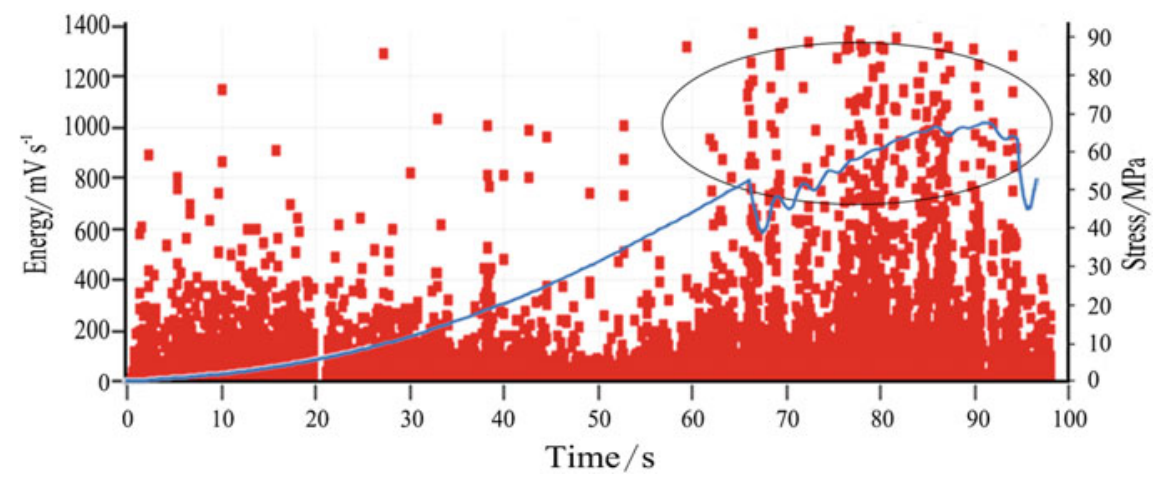

Fig. 6.9 Relationship between AE and stress with time. From Wang et al. (2015a, b) 


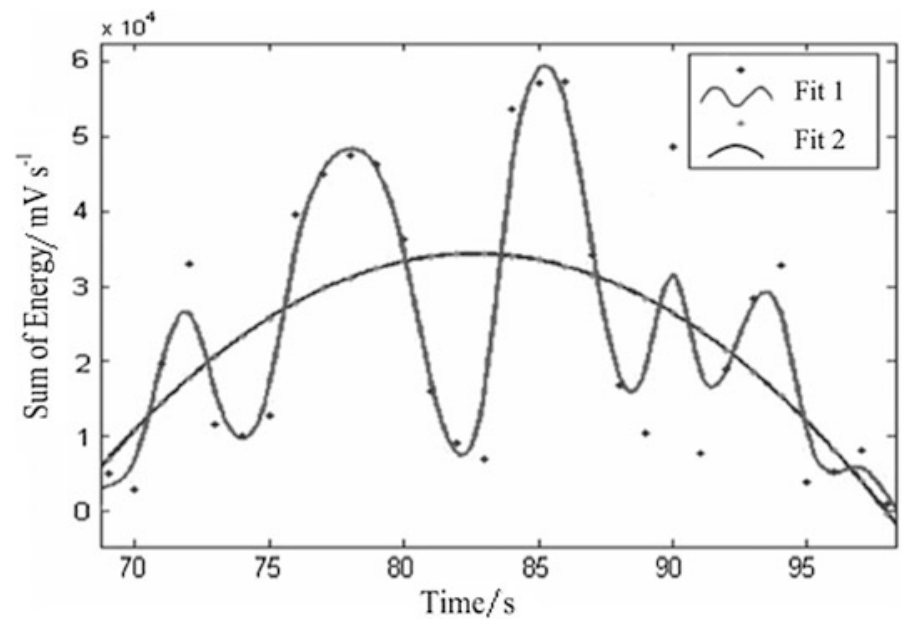

Fig. 6.10 Fitted curves of energy summation. From Wang et al. (2015a, b)

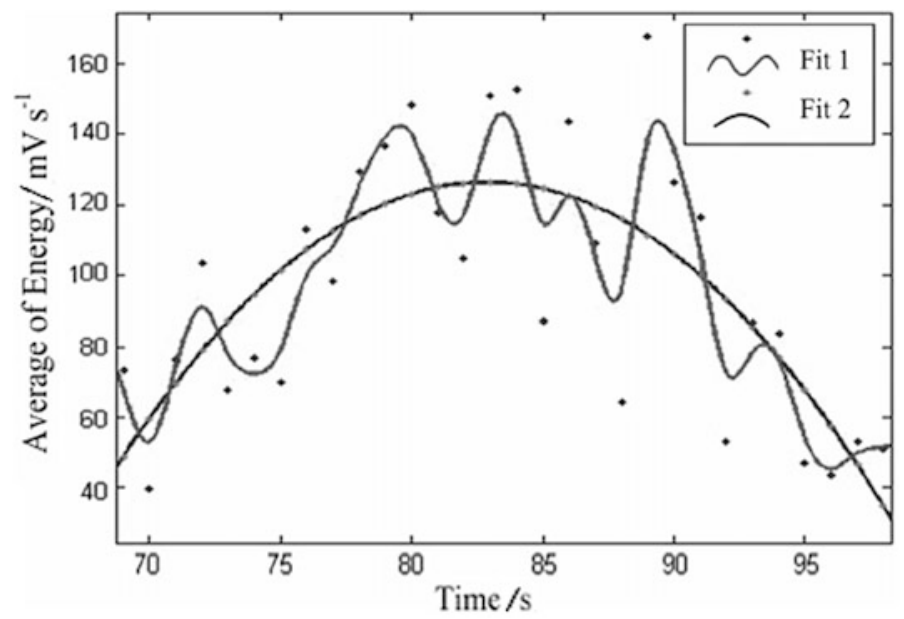

Fig. 6.11 Fitted curves of average energy. From Wang et al. (2015a, b)

curves, we could find that the energy values reached the maximum before $90 \mathrm{~s}$, indicating the maximum release of $\mathrm{AE}$ energy was before rock failure. Therefore, we estimated rock stable status by observing the variation of three indexes simultaneously. Compared with single energy index, we could more exactly judge rock energy release. Corresponding the fitted equations $f(\mathrm{t})$, summation of energy was obtained based on the experimental data as presented in Eq. (6.4). From derivation, the time of extreme values in the parabolic curves are $t_{1}, t_{2}$ and $t_{3}$, which could be used as the critical times (Wang et al. 2015a, b). 


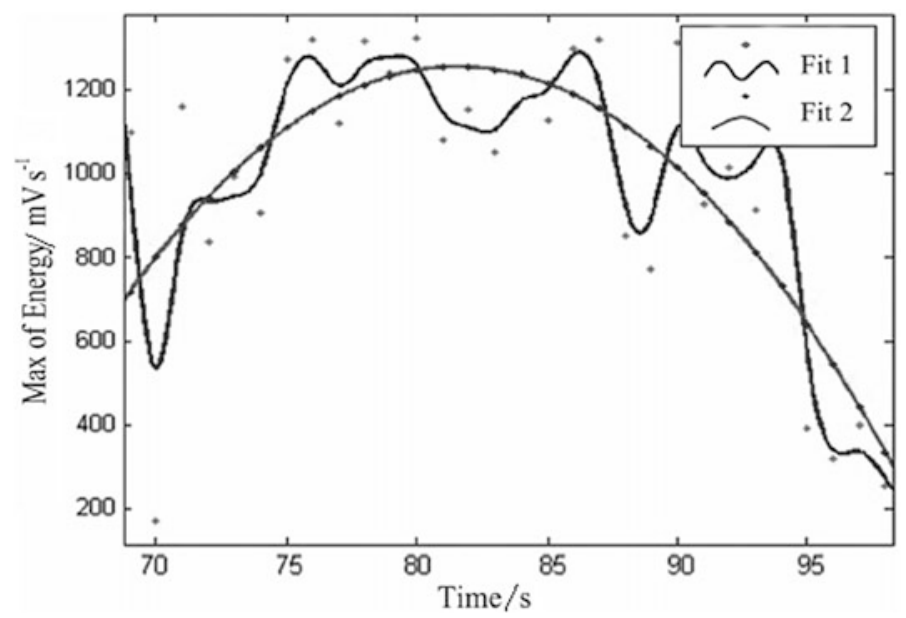

Fig. 6.12 Fitted curves of maximum energy. From Wang et al. (2015a, b)

The fitted curve of energy summation could be described as follow:

$$
f(t)=-147.2 t^{2}+24,330 t-971,000
$$

The time of the maximum value in energy summation curve could be obtained by the derivative of Eq. (6.5):

$$
f^{\prime}(t)=-294.4 t+24,330
$$

where $f^{\prime}(t)=0$, so $t_{1}=82.64$ s.

Based on the calculations above, the time of average energy, and the maximum energy could be obtained as $t_{2}=82.89 \mathrm{~s}$ and $t_{3}=81.57 \mathrm{~s}$. Calculated the average of $t_{1}, t_{2}$ and $t_{3}$ was $82.37 \mathrm{~s}$. It was obvious to find that the times of three indexs almost reached at the same time. Meanwhile, it was thought that the time of most intense $\mathrm{AE}$ energy release occurred around $82.37 \mathrm{~s}$ and regarded the point of time as a critical feature to predict rock failure (Wang et al. 2015a, b).

\subsubsection{Energy Release Rate of Rock Damage}

As shown in Fig. 6.9, as lots of scattered AE signals released, AE energy was always in dynamic evolution during the entire loaded process. Thus, it was hard to exactly describe the real energy release and the actual damage level. Then the concept of energy release rate was proposed, which referred to AE energy released per unit time, and eliminated the instability of AE release. Thereby, it was significative to further study the mechanism of energy release rate and analyze its reasonability. 
Being the numerical signal, AE energy is a quantitative description of continuous $\mathrm{AE}$ signals. From electronics theory, $\mathrm{AE}$ energy release rate is proportional to mean square voltage of $\mathrm{AE}$ signals, which means that the voltage can replace $\mathrm{AE}$ energy to describe the damage variation. Mean square voltage to calculating AE energy can be formulated as follows:

$$
E=\int_{t_{1}}^{t_{2}} V_{m s} d t
$$

Furthermore, the energy release rate can be calculated as:

$$
E^{\prime}=\frac{d E}{d T}=V_{m s}
$$

and the mean square voltage is calculated as

$$
V_{m s}=\frac{1}{\Delta T} \int_{0}^{\Delta T} V^{2}(t) d t
$$

where $V(t)$ is voltage of AE signals; $\Delta T$ is mean time interval. Energy release rate is expressed by the mean square voltage, as shown in Eq. (6.8).

Thus, the damage stability of rock can be judged by analyzing the energy release rate. Meanwhile, there is a positive correlation between mean square voltage and energy release rate. Therefore, the evolution process can be directly described by the variation of voltage to provide information or predicting of rock failure (Wang et al. 2015a, b).

\subsection{Evolution Patterns of Spatial-Temporal-Energy on Rock Fracture Surface}

Macroscopic failure of rock materials is the result of initiation, propagation and coalescence of rock cracks with a rapid release of transient elastic wave (AE). Much work of rock mechanics behavior and damage evolution were studied, and several valuable experimental results were obtained (Vidya Sagar et al. 2013). However, it is not comprehensive to study the evolution of rock failure process only from the features and roughness of fracture surface, rock failure process is a change status by energy release. Therefore, some work about energy point of rock failure were studied and several valuable experimental results were obtained (Peng et al. 2015). Wasantha et al. (2014) investigated the pattern of energy release during deformation of dry sandstone and saturated sandstone with different bedding directions under uniaxial compression. Carpinteri et al. (2006) studied the energy and dissipation 
energy of $\mathrm{AE}$ in the loading process of three point bending test of concrete beams in detail. Meanwhile, as we know, fracture surface is the main carrier of deformation and failure process; there are few researches which consider the relationship between fracture surface and energy of AE. Therefore, in this chapter, failure process of rock was studied through analyzing the changing pattern of time, space and energy of $\mathrm{AE}$ event on the fracture surface.

In this chapter, the spatial-temporal-energy evolution model of AE events on the fracture surface was established during rock failure, and the characteristics of fracture surface and the energy evolution were obtained during failure process, it provided us with scientific evidence for predicting rockburst.

\subsubsection{Experimental Setup and Procedure}

In this chapter, limestone samples were taken from the roof of coal seam at $1212 \mathrm{~m}$ depth in Xiezhuang Mine in Shandong province. These samples were processed as standard cylindrical rock samples of $50 \mathrm{~mm}$ in diameter and $100 \mathrm{~mm}$ in length. The experiment was adopted the axial displacement loading method, and the loaded rate was controlled at $0.005 \mathrm{~mm} / \mathrm{s}$. The loading machine and $\mathrm{AE}$ motoring system was operated synchronously.

The preamplifiers of $\mathrm{AE}$ were set at $40 \mathrm{~dB}$ before the samples were loaded. However, reducing the influence of environment noise, the threshold value of PCI was set at $45 \mathrm{~dB}$, and sampling rate was set at $1 \mathrm{MHz}$. AE testing system with 6 sensors for realizing real-time 3-D positioning of limestone samples during the entire deformation and fracture process. To reduce the influence of end-effects, 6 AE sensors were fitted on the limestone specimen $20 \mathrm{~mm}$ away from top and bottom of sample. Petroleum jelly and the adhesive band were used to fix the sensors on specimen surface. After the experiment, the relevant parameters of each event were analyzed during the entire process by Geiger algorithm, such as the $\mathrm{AE}$ rate, $\mathrm{AE}$ energy, $\mathrm{AE}$ position, and $\mathrm{AE}$ frequencies.

\subsubsection{Relationship Between Spatial Distribution of AE Events and Rock Fracture}

To study the evolution of spatial-temporal-energy evolution in the course of limestone failure, a series of $\mathrm{AE}$ tests were carried out for limestone failure under uniaxial compression. Stress strain data and AE data were obtained. Geiger algorithm was used to analyze $\mathrm{AE}$ events during loading process, and obtain the parameters of each event (time, position, energy). The corresponding parameters of $\mathrm{AE}$ events during the loading process of $\mathrm{X} 3$ rock sample were illustrated in Table 6.1. 
Table 6.1 AE events parameters of limestone sample X3 during the loading period

\begin{tabular}{l|l|l|l|l|l}
\hline \multirow{2}{*}{$\begin{array}{l}\text { Events } \\
\text { ID }\end{array}$} & \multirow{2}{*}{ Time } & \multicolumn{3}{|l|}{ Position Coordinates } & \multirow{2}{*}{ Energy } \\
\cline { 3 - 5 } & & $\mathrm{X}$ & $\mathrm{Y}$ & $\mathrm{Z}$ & \\
\hline 1 & 0.27 & -3.21 & 8.23 & 5.19 & 16 \\
\hline 2 & 0.29 & -12.18 & 10.16 & 15.13 & 27 \\
\hline 3 & 0.50 & -1.56 & -3.27 & 36.80 & 37 \\
\hline 4 & 0.73 & -9.22 & -11.06 & 36.79 & 19 \\
\hline 5 & 0.74 & 3.46 & -18.6 & 41.23 & 30 \\
\hline 6 & 0.84 & -7.58 & 8.48 & 21.17 & 51 \\
\hline 7 & 1.27 & 0.93 & -19.27 & 40.59 & 1 \\
\hline$\ldots$ & $\ldots$ & $\ldots$ & $\ldots$ & $\ldots$ & $\ldots$ \\
\hline$\ldots$ & $\ldots$ & $\ldots$ & $\ldots$ & $\ldots$ & $\ldots$ \\
\hline$\ldots$ & $\ldots$ & $\ldots$ & $\ldots$ & $\ldots$ & $\ldots$ \\
\hline 934 & 3.22 & -3.34 & -4.48 & 36.78 & 6 \\
\hline 935 & 3.68 & -13.06 & -9.25 & 36.73 & 2 \\
\hline 936 & 3.76 & -10.22 & -12.13 & 38.79 & 3 \\
\hline
\end{tabular}

A large number of $\mathrm{AE}$ events were obtained immediately, due to the internal deformation and cracks propagation. AE events reached several thousand or even tens of thousands during a loading process, if all the events were analyzed, then the workload was too great. Many small energy events could be ignored in the rock failure process. As a dominant reason for the ultimate failure of rock, which could only be large energy events. So, a large number of AE events were screened for energy, and finally the appropriate threshold of energy was determined. AE events over threshold were defined as a strong $\mathrm{AE}$ event. Comparison of $\mathrm{AE}$ spatial distribution and actual rupture propagation before and after the screening of X3 rock sample was demonstrated in Fig. 6.13.

Comparison of $\mathrm{AE}$ events spatial distribution and actual fracture propagation before and after the screening of X3 rock sample was shown in Fig. 6.13. As shown in Fig. 6.13b, AE events could be better reflected the evolution characteristics of internal rupture. There were many AE events in Fig. 6.13a, which leaded to the chaotic distribution of $\mathrm{AE}$ events in rock sample, and it was difficult to determine the specific location of main fracture surface. As shown in Fig. 6.13b, compared with the actual fracture propagation, $\mathrm{AE}$ events were mainly distributed near the main fracture surface of rock sample, which could clearly reflect the initiation, propagation and coalescence process of main fracture surface. Therefore, it is reasonable to study the spatial-temporal-energy evolution characteristics of $\mathrm{AE}$ in the loading process using energy screening method. 


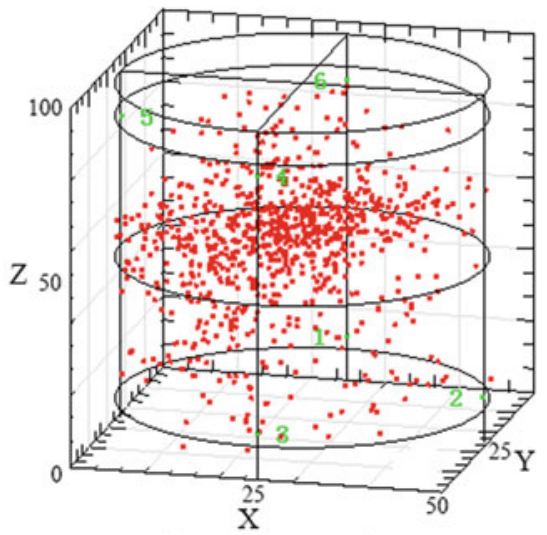

(a) Before screening

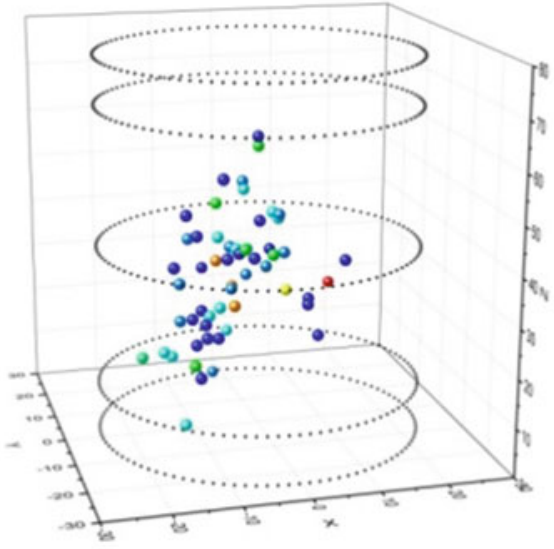

(c) AE events characterized by energy

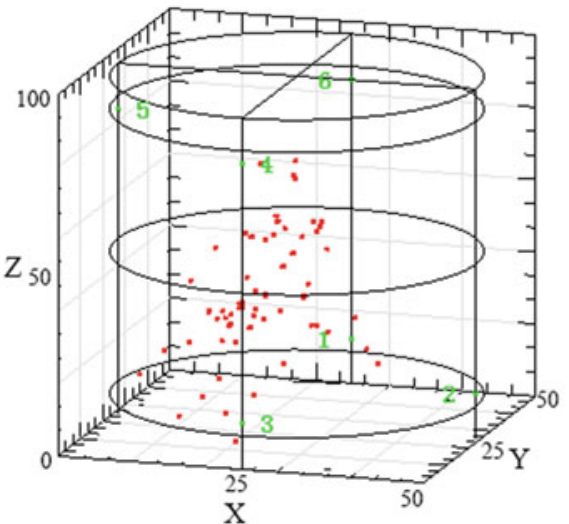

(b) After screening

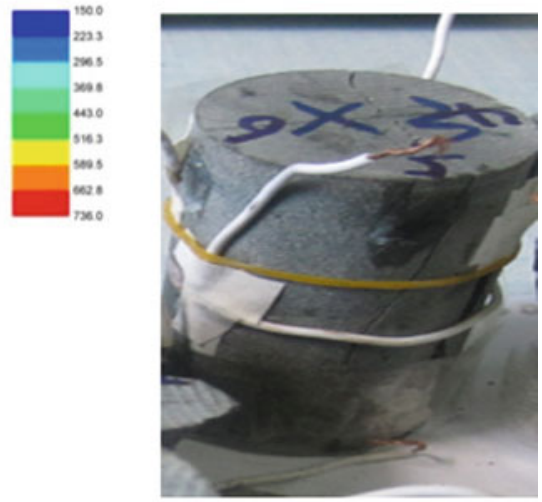

(d) actual rupture propagation

Fig. 6.13 Comparison of AE spatial distribution and actual rupture propagation

\subsubsection{Spatial-Temporal Evolution of Strong AE Events on Rock Fracture}

It was found that $\mathrm{AE}$ spatial distribution contained full information about internal deformation characters and cracks evolution of rock failure. Therefore, it provided a basis for studying the propagation process and spatial morphology of rock during loading process. AE localization of sample X3 in different loading period under uniaxial compression was shown in Figs. 6.14 and 6.15, where the color represented the magnitude of events energy value.

Analyzing the spatial-temporal-energy evolution characteristics of strong AE events, the spatial-temporal-energy evolution model of rock fracture surface was established. In this chapter, using the energy screening methods, it was showed that 


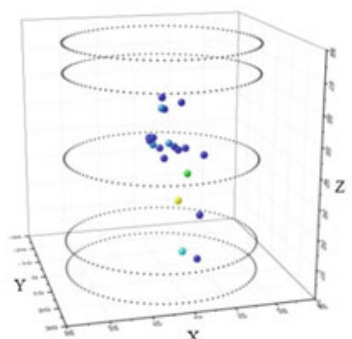

(a) $20 \% \sigma c$

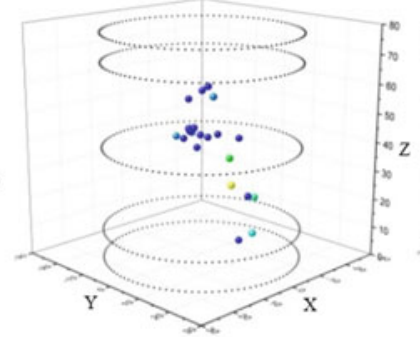

(b) $40 \% \sigma \mathrm{c}$

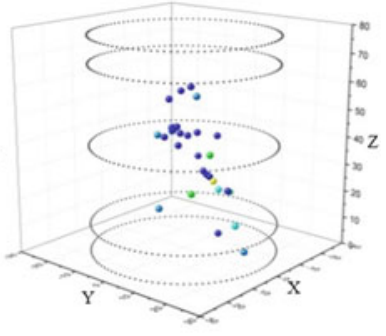

(c) $60 \% \sigma \mathrm{c}$

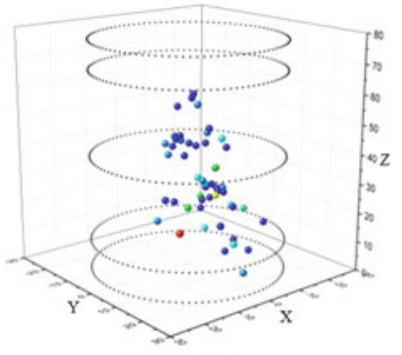

(d) $80 \%$ бc

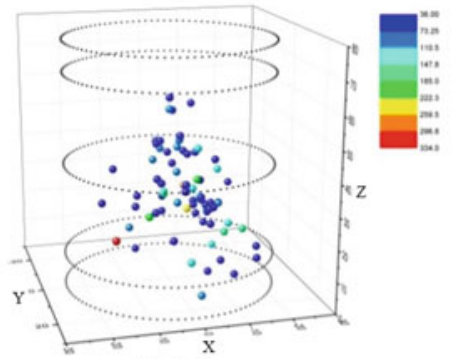

(e) $100 \% \sigma_{c}$

Fig. 6.14 AE localization of sample $X 3$ in different loading period

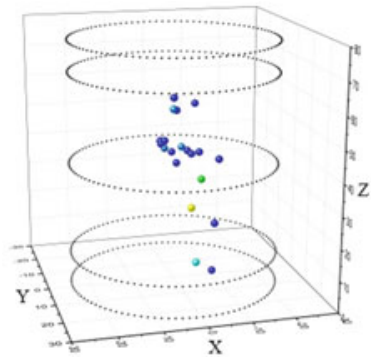

(a) $0 \sim 20 \%$ бc

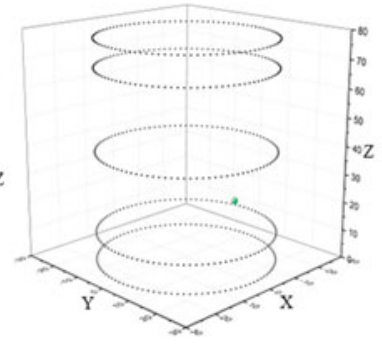

(b) $20 \% \sim 40 \%$ бc

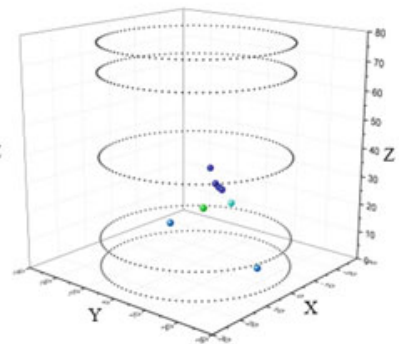

(c) $40 \% \sim 60 \% \sigma \mathrm{c}$

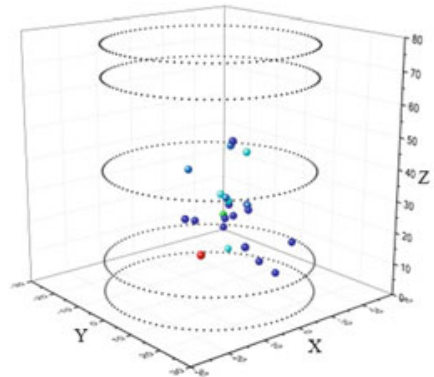

(d) $60 \% \sim 80 \% \sigma \mathrm{c}$

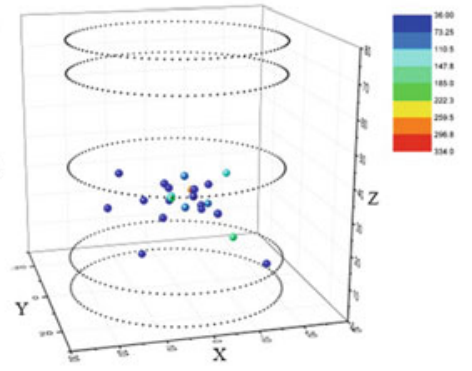

(e) $80 \% \sim 100 \%$ бc

Fig. 6.15 AE localization of sample $\mathrm{X} 3$ in different loading period (piecewise representation) 
most of strong AE events were distributed on rock fracture surface. As a matter of fact, the weak AE events had little effect on rock failure, and the occurrence of strong AE events corresponded to damage deformation. Therefore, it could be better analyzed the spatial position, propagation direction and spatial shape of cracks propagation in rock samples, and it could be more accurately determine the location of fracture surface, so as to provide predictions for the final rupture of rock condition.

\subsubsection{Spatial-Temporal-Energy Evolution Model of Strong $A E$ Events in Limestone Fracture}

As shown in Fig. 6. 16, it was the relationship curves between cumulative energy of strong AE events and time in the fracture surface of rock samples during the loading process. Take X3 rock sample for example, when stress was less than $20 \%$ of the peak stress, in the fracture compaction stage, more low energy events were monitored in the lower part of rock sample, and the middle energy events were relatively large in the lower part, which may be the internal joint fracture. The lower part was more developed, under the action of external load joints began to close, resulting in AE events, and activities were more intense. With the stress increased, rock sample entered the cracks initiation stage, and a small amount of low energy AE events were observed at the top and bottom of rock sample. The fracture surface did not form at this stage. When stress reached at 40-60\% of the peak stress, rock specimen entered cracks in the stable development stage, and $\mathrm{AE}$ events were significantly increased in the lower part of rock sample, event energy was larger, forming the strip shape with the pre-generated event. When stress reached at $80 \%$ of the peak stress, rock sample entered the unstable development stage of cracks, AE events increased rapidly and energy increased significantly. AE events on the strip began to be concentrated, and then internal cracks began to pass through, forming macro-cracks, and fracture surface was formed. In the later of this stage, number of $\mathrm{AE}$ events and the accumulated energy hardly increased, and there was a brief silence zone, namely, AE "quiet period", which could be used as a predicting point of rock destabilization failure (Wang 2015b). When stress exceeded the peak stress and entered the failure stage, AE event was obviously reduced compared to the previous stage. The macro fracture connection and coalescence of rock sample form the main fracture surface, and the rock sample was failure.

From the above analysis, it could be seen found that the formation of fracture surface mainly concentrated on the plastic stage and the failure stage. Therefore, the pattern of energy evolution pattern of rock fracture surface should begin with the plastic stage, which could be better revealed the evolution pattern of propagation and coalescence of internal cracks in rock samples.

The cumulative energy of plastic section of each rock sample, the abscissa was as the time, and the ordinate was selected as the cumulative energy. The Gauss 

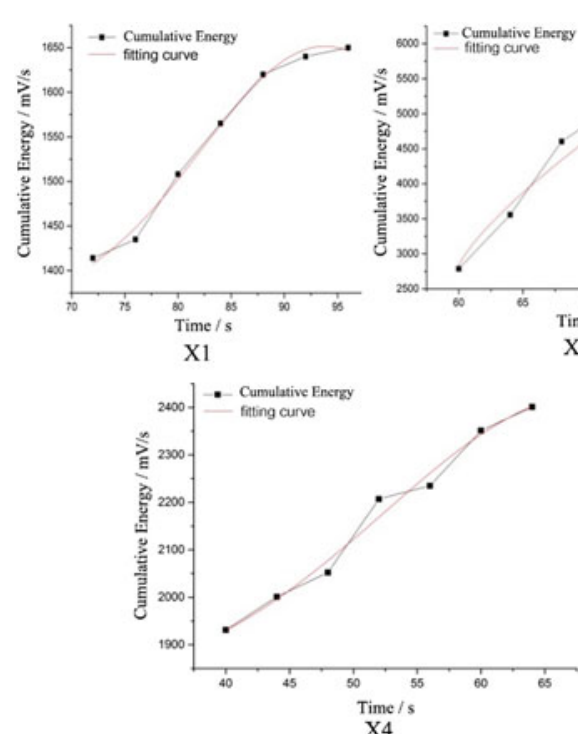
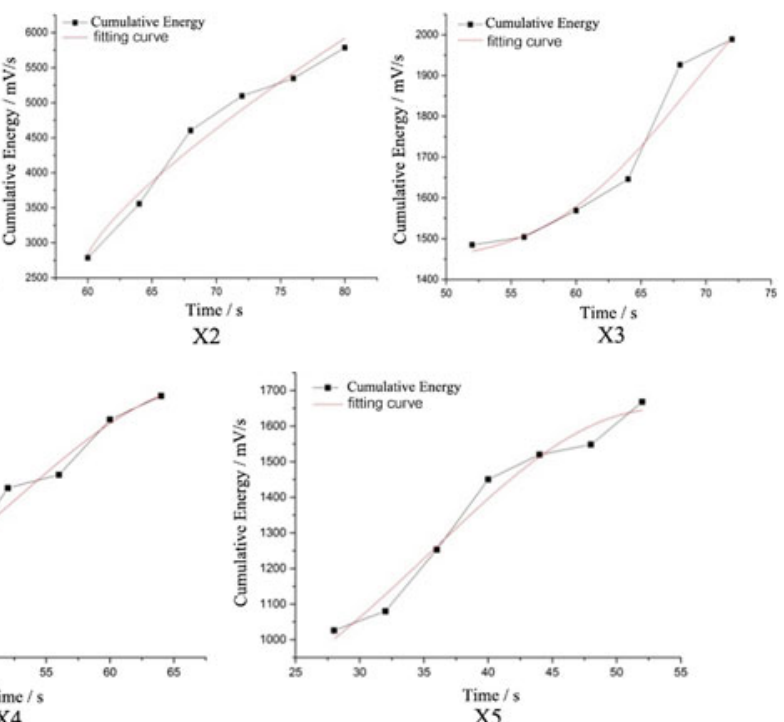

Fig. 6.16 Relationship curves between cumulative energy of strong AE events and time in the fracture surface

Amp function was used to fit, and the fitting function was $y=y_{0}+A e^{-\frac{\left(x-x_{c}\right)^{2}}{2 \omega^{2}}}$, the dependent variable $y$ was used to represent the cumulative AE energy, $x_{c}$ was used to represent the critical moment, $y_{0}$ was used to represent cumulative AE energy in the plastic stage, A was used to represent the amplitude of cumulative AE energy, $\omega$ was the relevant parameters, and it could be satisfied the equation $2 \omega=F W H M / \operatorname{sqrt}(\ln (4))$, which could calculate the value of $\omega$. So the original formula changes to $E=E_{0}+A \mathrm{e}^{-\frac{\left(t-t_{c}\right)^{2}}{2 \omega^{2}}}$. The obtained AE data were analyzed in the experiment, and the cumulative energy-time curve of strong event in fracture surface was plotted. Then the above functions were used to fit, the fitting results were shown in Fig. 6.16, and the related parameters were shown in Table 6.2.

Table 6.2 Related parameters and equations of fitting curves

\begin{tabular}{l|l|l|l|l|l}
\hline \multirow{2}{*}{ Specimen ID } & \multicolumn{4}{l}{ Related parameters } & \multirow{2}{l}{ Fitting equations } \\
\cline { 2 - 6 } & $\mathrm{E}_{0}$ & $\mathrm{~A}$ & $\mathrm{t}_{\mathrm{c}}$ & $\omega$ & \\
\hline $\mathrm{X} 1$ & 1357 & 293 & 93.8 & 11.6 & $E=1357+293 \mathrm{e}^{\frac{(t-9.3 .8)^{2}}{2 \times 11.6)^{2}}}$ \\
\hline $\mathrm{X} 2$ & 1415 & 4223 & 78.3 & 15.8 & $E=1415+4223 \mathrm{e}^{\frac{(t-78.3)^{2}}{2 \times 15.8^{2}}}$ \\
\hline $\mathrm{X} 3$ & 1443 & 684 & 79.1 & 10.6 & $E=1443+684 \mathrm{e}^{-\frac{(t-79.1)^{2}}{2 \times 10.6^{2}}}$ \\
\hline $\mathrm{X} 4$ & 1797 & 630 & 79.5 & 16.2 & $E=1443+684 \mathrm{e}^{\frac{(t-79.1)^{2}}{2 \times 10.62^{2}}}$ \\
\hline $\mathrm{X} 5$ & 833 & 806 & 53.5 & 17.9 & $E=833+806 \mathrm{e}^{-\frac{(t-53.5)^{2}}{2 \times 17.9)^{2}}}$ \\
\hline
\end{tabular}




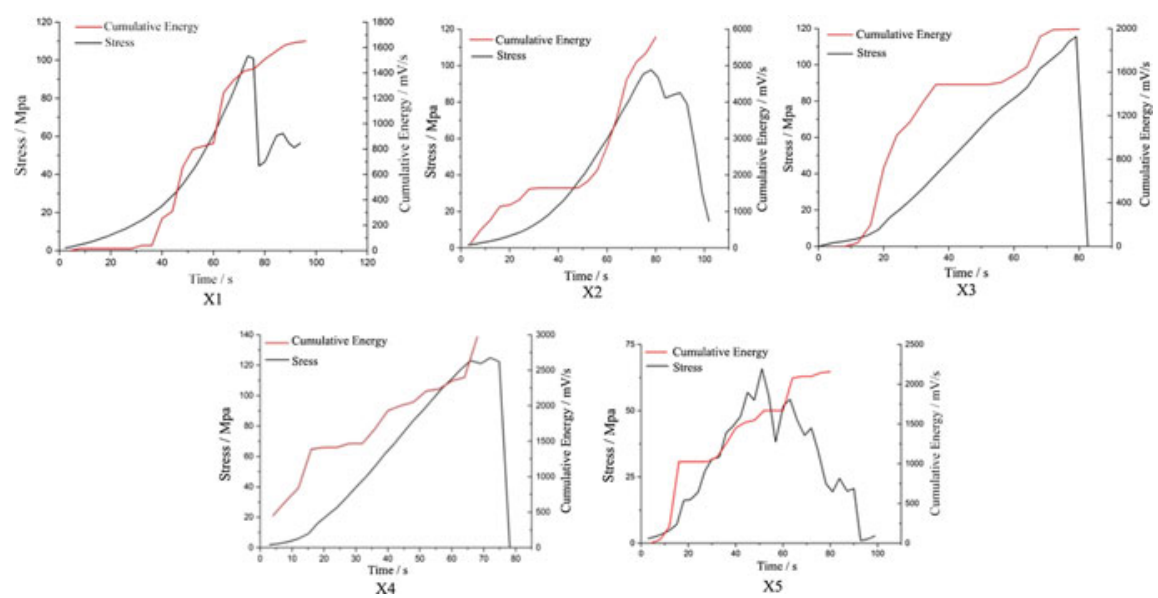

Fig. 6.17 Relationship fitting curves between cumulative energy of strong AE events and time in the fracture surface

As shown in Fig. 6.17, besides the rock samples X3 and X5 (the correlation coefficient of X3 is 0.93 and X5 is 0.91 ), the fitting correlation coefficients of other 3 rock samples were all above 0.97. The results showed that Gauss Amp function fitted well, and the parameters $E_{0}, A, t_{c}$ and $\omega$ could be obtained by fitting the results.

During the loading process, the input energy was mainly converted into two parts: one part was dissipated in the form of damage energy and plastic deformation energy of rock specimen. The other part was accumulated inside the rock sample in the form of elastic strain energy and could be released during unloading. The ratio of elastic energy and dissipated energy to input energy had a great influence on rock deformation and failure modes. As a part of dissipation energy, AE energy might be related to the external input energy. The area between stress-strain curve and transverse axis was the strain energy density. The related parameters of samples energy were shown in Table 6.3.

As shown in Table 6.3, vary of strain energy density and cumulative AE energy was showed at the plastic stage. However, when strain energy density was large,

Table 6.3 Related parameters of samples energy

\begin{tabular}{l|l|l|l}
\hline $\begin{array}{l}\text { Specimen } \\
\text { ID }\end{array}$ & $\begin{array}{l}\text { Strain energy } \\
\text { density }\end{array}$ & $\begin{array}{l}\text { Cumulative AE energy at the plastic } \\
\text { stage }\end{array}$ & $\begin{array}{l}\text { Proportion } \\
(\%)\end{array}$ \\
\hline $\mathrm{X} 1$ & 0.266419 & 1357 & 0.51 \\
\hline $\mathrm{X} 2$ & 0.214304 & 1415 & 0.66 \\
\hline $\mathrm{X} 3$ & 0.273068 & 1443 & 0.53 \\
\hline $\mathrm{X} 4$ & 0.354202 & 1797 & 0.51 \\
\hline $\mathrm{X} 5$ & 0.118236 & 833 & 0.70 \\
\hline
\end{tabular}


cumulative AE energy corresponding was correspondingly large. Its proportion in a certain range ranged from 0.5 to $0.7 \%$.

In conclusion, it could be concluded that cumulative AE energy increased with time during the failure process. Especially, after the plastic stage, cumulative AE energy of strong events in the fracture surface conformed to Gauss Amp function distribution. Therefore, the spatial-temporal-energy evolution model of limestone on the fracture surface was proposed:

$$
E=k \int_{0}^{\varepsilon_{c}} \sigma d \varepsilon+A \mathrm{e}^{-\frac{\left(t-t_{c}\right)^{2}}{2 \omega^{2}}}
$$

where $k$ is a material constant, and its value is between 0.5 and $0.7 \% ; t_{c}$ is the critical moment; $t$ is the loading time, and it ranges from the initial stage of plastic stage to the peak stress; $\varepsilon_{c}$ is strain corresponding to peak stress; $\omega$ is the coefficient in Gauss Amp function; $A$ is the increment of cumulative AE energy from the beginning of plastic phase to the peak stress; $E$ is cumulative AE energy of strong events.

Energy of AE events directly characterized the intensity of internal mesoscopic damage, and the weak event had little effect on rock failure, and the ultimate failure of rock was dominated by strong event. On the basis of predecessors, energy was taken into account in the spatial-temporal evolution analysis of AE events, so as to analyze the spatial-temporal-energy evolution of AE events on the fracture surface. The proposed spatial-temporal-energy evolution model combined the spatial-temporal events and energy of fracture surface in the process of limestone rupture and failure, and it described the energy evolution pattern in the formation of fracture surface under the uniaxial compression. The model was used to describe the energy change process of fracture surface systematically, and it provided a theoretical basis for predicting rockburst. However, this model is only applicable to the uniaxial compression test of limestone, and further study is needed for other rock and other stress conditions.

\subsubsection{Spatial Fractal Dimension Evolution Model of AE Events on Fracture}

In 1973, mathematician Mandelbrot first proposed the idea of using fractional and fractal geometry to describe irregular objects and chaotic phenomena in nature (Mandelbrot 1983). Analyzing AE signal produced in the propagation of micro-cracks, it was found that AE parameters followed not only fractal characteristics in time, but also fractal characteristic in spatial distribution. The balls covering method or the circle covering method after projection were widely used for three-dimensional distribution of AE events. For the cylindrical rock specimens, 
column covering method was more appropriate. In this method, the spatial distribution characteristics of AE events were apt to be described exactly. Due to the shape of rock specimens, it was very convenient for the method to calculate the fractal dimension of spatial $\mathrm{AE}$ event distribution according to the coordinates of AE events. Therefore, the method could be used to determine the critical value of rock failure, predict the rockbursts, and evaluate the stability of rockmass.

When AE events were covered by a cylinder with radius $r$ and height $h$, the relationship between $\mathrm{AE}$ event number and the radius of cylinder was:

$$
M_{(r)} \propto r^{2} h \propto C_{1} r^{3}
$$

where $C 1$ represents height-radius ratio. Equation (6.10) could be further expressed as:

$$
\log M_{(r)}=\log C+\log C_{1}+D \log r
$$

where $r$ is a given radius, $M(\mathrm{r})$ is a value which can be obtained through Eq. (6.11). In a double logarithmic coordinate system, point series (log $r, \log \mathrm{M}(\mathrm{r})$ ) corresponding to given radii can be portrayed. The spatial AE event distribution has fractal characteristic in the range of a given scale if those points approximately distribute along a straight line. The slope of line is the exact fractal dimension of spatial AE event distribution.

Based on the above-mentioned column covering method, using the different radius $r$ of the cylinder to cover the strong AE events, the relationship between number of strong events $\mathrm{M}(\mathrm{r})$ and radius was obtained. In a double logarithmic coordinate system, point series $(\log r, \log \mathrm{M}(\mathrm{r}))$ corresponding to given radii could be portrayed. Then the linear point of data points was fitted, and the obtained slope was the fractal dimension of spatial distribution of large events in rock sample under the stress level. The curve between the spatial fractal dimension and the stress level of strong AE events of limestone rock samples was shown in Fig. 6.18.

Fig. 6.18 Relationship curves between spatial fractal value of $\mathrm{AE}$ events dimensional distribution and stress

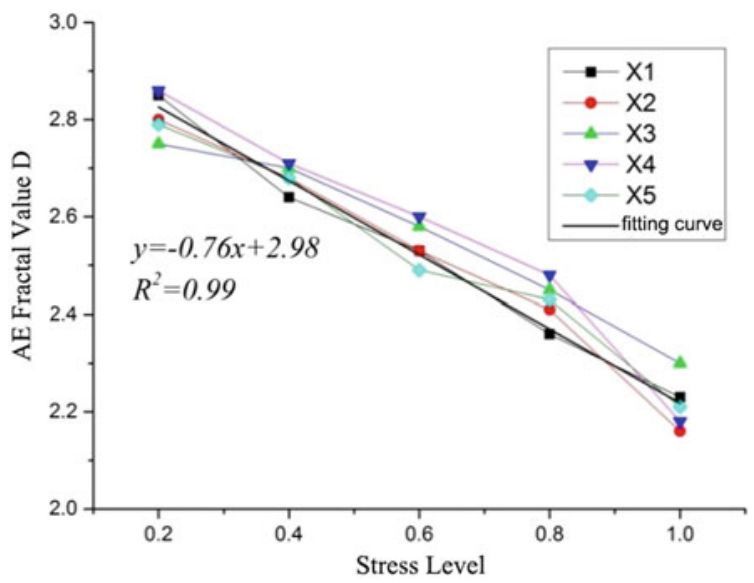


When stress was at a low level (below $0.2 \sigma_{\mathrm{c}}$ ), the spatial fractal dimension, close to 3, was relatively large indicated that $\mathrm{AE}$ events in this stage were more evenly distributed in the interior space of rock samples. With the increase of stress, rock sample entered the cracks initiation stage and cracks stabilization expansion stage $\left(0.2-0.7 \sigma_{\mathrm{c}}\right)$. Because of less AE events, $D$ value of this stage decreased slowly. With the increase of stress, rock specimen entered the unsteady expansion stage $\left(0.7-0.9 \sigma_{\mathrm{c}}\right)$, the internal damage increased and the fractal dimension decreased continuously. When stress value reached about $80 \%$ of the peak stress, $D$ value decreased rapidly, indicated that the internal events began to accumulate near the macroscopic main fracture surface. When stress reached the peak stress, the macro-fracture surface was formed gradually, and $D$ value dropped to the minimum value, which was close to 2.2 .

By analyzing the relationship curve between the spatial fractal dimension and the stress level of AE event, the spatial fractal dimension decreased linearly with the increase of stress level. Therefore, the least squares method was used to fit the curve, and the fitted curve was shown in Fig. 6.18. The fitted equation was as follow:

$$
D=-0.76 \frac{\sigma}{\sigma_{c}}+2.98
$$

where $D$ is the spatial fractal dimension, and its value is between 2 and $3 ; \sigma_{c}$ is the peak stress; $\sigma$ is the stress value during loading process.

\subsection{Conclusion}

Rock failure was accompanied by changing of AE parameters as well. In view of this issue, this section was built on the laboratory test, explored and quantized the feature of failure precursory information, and obtained the following conclusions:

(1) Though the distribution was very heterogeneous, through analyzed the feature of spatio-temporal evolution in the different loading stage, we still found two main phenomenons after summarizing all sample results: intensive phenomenon and zonation.

(2) The characteristic point of the relatively quiet period was defined as a characteristic precursor point of rock failure. The ratio of stress at the relatively quiet period was from 88 to $98 \%$ of the peak stress in the unstable propagation stage.

(3) In the plastic stage, AE energy rapidly increased and remained at high values indicating intense energy release before rock failure. In the failure stage, AE energy sharply decreased and rock instability occurred. The corresponding time, when AE energy release rate reached at the maximum, can be suggested as the critical point for predicting rock failure. Meanwhile, AE energy release rate could be considered as an important rock failure index, which might provide physical basis for predicting rockburst. 
(4) Strong AE events could fully reflect the evolution of internal damage and deformation, and were concentrated in the vicinity of main fracture surface. Through the spatial distribution analysis of strong AE events, the position of rupture surface could be determined more accurately.

(5) The range of fractal dimension of strong AE event was from 2 to 3 , the value of the initial stage was close to 3 , and the value of final failure stage was close to 2 . The spatial fractal dimension showed a sharp decrease near the yield point (about $0.8 \sigma_{\mathrm{c}}$ ), indicating that rock was about to lose stability and could be used as a precursory feature of rockburst. Moreover, there was a linear relationship between fractal dimension and stress level.

(6) The fracture surface was mainly produced in the plastic stage and the failure stage under uniaxial compression. Cumulative AE energy of fracture surface conformed to Gauss Amp function distribution. Therefore, the spatial-temporal-energy evolution model in the fracture surface for strong AE event was established.

\section{References}

Carpinteri A, Lacidogna G (2006) Monitoring the durability performances of concrete and masonry structures by acoustic emission technique. In: Fracture of nano and engineering materials and structures, pp 781-782

Carpinteri A, Lacidogna G, Paggi M (2007) Acoustic emission monitoring and numerical modeling of FRP delamination in RC beams with non-rectangular cross-section. Mater Struct 40:553-566

Mandelbrot BB (1983) The fractal geometry of nature. New York: WH freeman

Lacidogna G, Carpinteri A, Manuello A, Durin G, Schiavi A, Niccolini G, Agosto A (2011) Acoustic and electromagnetic emissions as precursor phenomena in failure processes. Strain 47:144-152

Mandelbrot BB (1983) The fractal geometry of nature. New York: WH freeman

Meng Q, Zhang M, Han L, Pu H, Nie T (2016) Effects of acoustic emission and energy evolution of rock specimens under the uniaxial cyclic load/unload compression. Rock Mech Rock Eng 49:3873-3886

Miller, RK, Pollock, AA, Finkel P, Watts DJ, Carlyle JM, Tafuri AN, Yezzi, JJ (1999) The development of acoustic emission for leak detection and location in liquid-filled, buried pipelines. In: Acoustic emission: standards and technology update. ASTM International

Peng R, Ju Y, Wang JG, Xie H, Gao F, Mao L (2015) Energy dissipation and release during coal failure under conventional triaxial compression. Rock Mech Rock Eng 48:509-526

Vidya Sagar R, Prasad RV, Raghu Prasad BK, Rao MVMS (2013) Microcracking and fracture process in cement mortar and concrete: a comparative study using acoustic emission technique. Exp Mech 53:1161-1175

Wang C (2014) Identification of early-warning key point for rockmass instability using acoustic emission/micro seismic activity monitoring. Int J Rock Mech Min 71:171-175

Wang C, Bao T, Lu H, Liu L, Lu Z, Li W, Yu Q (2015a) Variation regulation of the acoustic emission energy parameter during the failure process of granite under uniaxial compression. Mater Test 57(9):755-760

Wang CL, Lu H, Wang FL et al (2015b) Characteristic point of the relatively quiet period for limestone failure under uniaxial compression. J Test Eval 43(6):1296-1307

Wasantha PLP, Ranjith PG, Shao SS (2014) Energy monitoring and analysis during deformation of bedded-sandstone: use of acoustic emission. Ultrasonics 54:217-226 
Open Access This chapter is licensed under the terms of the Creative Commons Attribution-NonCommercial 4.0 International License (http://creativecommons.org/licenses/by-nc/ 4.0/), which permits any noncommercial use, sharing, adaptation, distribution and reproduction in any medium or format, as long as you give appropriate credit to the original author(s) and the source, provide a link to the Creative Commons license and indicate if changes were made.

The images or other third party material in this book are included in the book's Creative Commons license, unless indicated otherwise in a credit line to the material. If material is not included in the book's Creative Commons license and your intended use is not permitted by statutory regulation or exceeds the permitted use, you will need to obtain permission directly from the copyright holder.

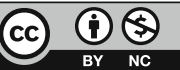




\section{Chapter 7 \\ Experimental Investigations \\ on Multi-means and Synergistic Prediction for Rockburst}

\subsection{Introduction}

Rock is a kind of complicated geological material with in-homogeneity, anisotropy and discontinuity (Senseny et al. 1992). Under external force, the mechanic behaviour of rock is relatively complex, and it is difficult to accurately predict rockburst (Cook 1992; Brady 1969). Rockburst can lead to damage of equipment, substantial staff casualties and property losses (Blake and Hedley 2003; He et al. 2010). Thus, it has become important question for scholars and engineers to be able to predict rockburst.

Many scholars had some research on predicting rockburst. Schiavi et al. (2011b) found that the phenomenon of a relatively quiet period appeared before rockburst under uniaxial compression. Carpinteri et al. (2006), Carpinteri and Lacidogna (2008) made testing on concrete, rock and other materials with AE monitoring technology, and the result demonstrated that AE energy accelerates release before rock failure. Wang et al. (2009) carried out a large number of AE tests in laboratory, and suggested that AE relatively quiet period could be regarded as a precursor. Meanwhile, he studied the fluctuation of $b$ value and obtained the precursory characteristic and predicting key point of rockburst (Wang 2014).

Read et al. (1995) analysed AE waveforms from a series of laboratory triaxial-deformation experiments, and explored the features of waveform amplitudes and frequency before and after the peak stress. Benson et al. (2010) found that disordered AE signals were significantly reduced before rock specimen failure. Schiavi et al. (2011a, b, 2013) founded that elastic emissions were different from acoustic emissions, could be detected in a low frequency range (below $15-10 \mathrm{kHz}$ ). He et al. (2015) presented an experimental investigation on the instantaneous strain-type granitic rockbursts, and found that frequencies changed from low value to high values as the load increased. Lu et al. (2012a, b, 2013) demonstrated that the dominant frequency of main shock signal correlate negatively with rockburst intensity. Kukshenko and Elizarov (2015) made AE test on large scale specimens,

C. Wang, Evolution, Monitoring and Predicting Models of Rockburst, 
and analyzed the result using the load/unload response ratio (LURR) theory, found that $Y$ value change of LURR was consistent with that of rock internal damage, and that the value of $Y$ value increased significantly when the integrity of rock samples was destroyed. LURR theory was also applied to evaluate the degree of damage, which provided a new approach to describe rock failure behavior (Shi et al. 1994; Zhang et al. 2013). Some results for earthquake precursor information were obtained, radiation temperatures in the earth's crust were unusually high in the weather satellites observation (Qiang et al. 1990). Before the earthquake, earth satellite images had obvious infrared thermal enhancement phenomenon, which was called "thermal anomaly" (Freund et al. 2007). In the past few decades, the abnormal increase of surface temperature was successfully verified (Genzano et al. 2007; Saraf et al. 2008). In laboratory tests, abnormal infrared radiation was used as precursor of rock failure on the Similarity simulation experiment of earthquake (Rawat et al. 2011; Naiguang et al. 1998; Geng et al. 1992).

The new inter-discipline of remote sensing rock mechanics (RSRM) was put forward, and it could serve in the forecasting of time-space location of potential rock failure events by IRR monitoring (Wu et al. 2000). Based on a series of experiments of IRR features for multiple rocks under different loading conditions, it was revealed that the detected temperature would reflect the IRR energy variation, and that the abnormal IRR precursors could provide forewarning information for rock failure (Wu et al. 2001; Cui et al. 1993; Geng et al. 1992). Wu et al. (2006a, b) found that thermogram abnormality and temperature curve abnormality were detected as precursors of rock fracture. Three precursor messages-short dropping, fast rising and dropping-to-rising were observed using the temporal evolution features of average infrared radiation temperature curves (Wu et al. 2006a, b).

It is difficult to carry out the experiment of rockmass load/unload condition on site due to the constraints of various actual conditions. Meanwhile, laboratory test methods can respond to engineering applications in a certain degree. Therefore, it is of great significance to make the research on rock failure predicting in the laboratory.

In this chapter, based on previous research results, we performed the tests in laboratory and research the precursory information before rockburst in field. At present, the technology of rock failure predicting is applied in a single, hysteresis and poor effect, which can not timely, comprehensively and effectively predict the hazards. Therefore, it is very important to establish a multi-means monitoring and cooperative predicting for rockburst. In this chapter, we studied on the predicting key point of rockburst used the stress-strain monitoring, AE monitoring, infrared monitoring, microseismic monitoring, et al. 


\subsection{Predicting Points of Infrared Precursor for Coal Failure}

\subsubsection{Laboratory Test of Coal Failure}

In this chapter, as shown in Fig. 7.1, coal specimens were cylindrical cores with a diameter of $50 \mathrm{~mm}$ and a length of $100 \mathrm{~mm}$. Two end planes of every specimen were parallel within an accuracy of $\pm 0.05 \mathrm{~mm}$, and both planes were perpendicular to the longitudinal axis with an accuracy of $\pm 0.25^{\circ}$, and actual size and mechanic parameters (Table 7.1).

$\mathrm{AE}$ sensors were glued approximately $20 \mathrm{~mm}$ from the two end planes to reduce the interference for $\mathrm{AE}$ data collection. The sampling frequency of sensor and specimen was $1 \mathrm{MHz}$, and $\mathrm{AE}$ signal acquisition threshold was set to $40 \mathrm{~dB}$. Based on coal specimen's ultimate compression strength (UCS), experiments were divided into three-section uniaxial cyclic load/unload process. Coal specimens were loaded at $200 \mathrm{~N} / \mathrm{s}$. In the first phase, coal specimens were loaded from 8 to $16 \mathrm{MPa}$ before being unloaded back to $8 \mathrm{MPa}$. In the second phase, coal specimens were loaded from 16 to $24 \mathrm{MPa}$ before being unloaded back to $16 \mathrm{MPa}$. In the third phase, coal

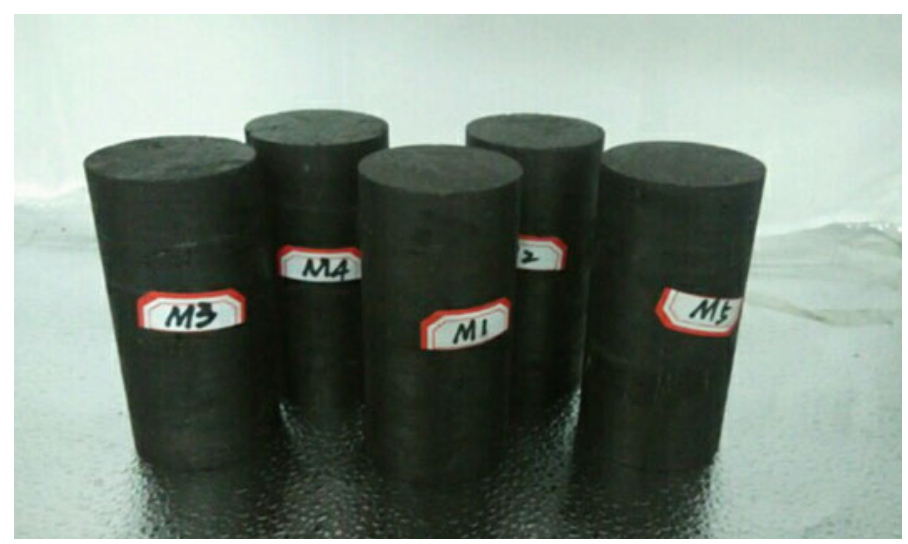

Fig. 7.1 Photos of coal specimens

Table 7.1 Summary of coal specimens

\begin{tabular}{l|l|l|l|l|l|l}
\hline $\begin{array}{l}\text { Specimen } \\
\text { ID }\end{array}$ & $\begin{array}{l}\text { Diameter } \\
(\mathrm{mm})\end{array}$ & $\begin{array}{l}\text { Height } \\
(\mathrm{mm})\end{array}$ & $\begin{array}{l}\text { Weight } \\
(\mathrm{g})\end{array}$ & $\begin{array}{l}\text { Density }(\mathrm{g} / \\
\left.\mathrm{cm}^{3}\right)\end{array}$ & $\begin{array}{l}\text { Elastic } \\
\text { modulus (Gpa) }\end{array}$ & $\begin{array}{l}\text { UCS } \\
(\mathrm{MPa})\end{array}$ \\
\hline M1 & 49.61 & 99.51 & 245.80 & 1.278 & 2.58 & 39.51 \\
\hline M2 & 49.66 & 99.25 & 245.34 & 1.276 & 2.36 & 27.84 \\
\hline M3 & 49.63 & 99.86 & 241.47 & 1.250 & 2.32 & 26.83 \\
\hline M4 & 49.95 & 99.18 & 272.68 & 1.403 & 2.93 & 28.60 \\
\hline M5 & 49.73 & 99.79 & 247.59 & 1.277 & 2.19 & 27.73 \\
\hline
\end{tabular}




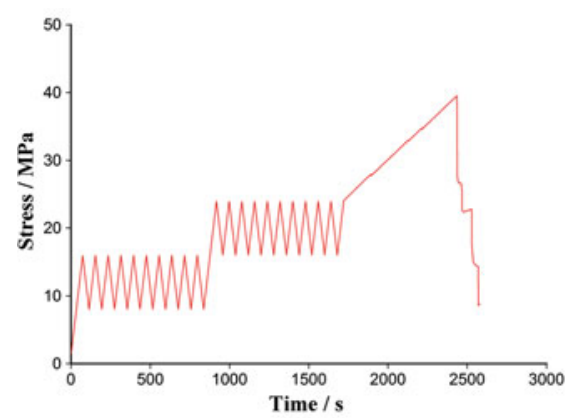

(a) Loading path.

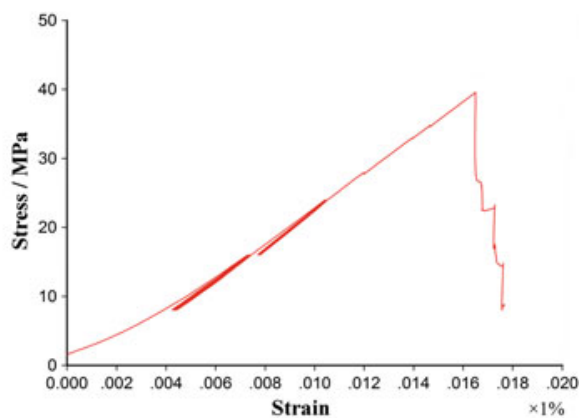

(b) Loading-unloading curve of the coal specimens.

Fig. 7.2 Testing curve and path for coal specimens

specimens were continuously loaded from $16 \mathrm{MPa}$ until failure. Cyclic loading path of coal specimens was shown in Fig. 7.2. AE monitoring was carried out real time dynamically during loading. Meanwhile, infrared radiation (IRR) experiments were be monitored.

Regard to the thermographic camera, the graphic recording rate was set to 4 frames per second. InfRec R300 directly faced rock sample surface approximately $1 \mathrm{~m}$ from samples. IRR on rock surface was debugged before rock samples were loaded. The ambient temperature and humidity in the laboratory were tested before the experiment. The ambient temperature was $22.10{ }^{\circ} \mathrm{C}$, and the humidity was $29 \%$. The emissivity of tested coal sample was 0.92. In the experiment, TAW-2000 and InfRec R300 were operated simultaneously during the whole deformation and fracture processes on the coal samples.

\subsubsection{Theoretical Bases of IRR Detection}

Temperature is a comparative objective parameter of hot and cold. It reflects the thermal state of object and can be measured in a contact or noncontact method. The noncontact mode is based on the measurement of IRR emitted from the object surface. Thermal radiation is the emission of electromagnetic waves from all matter with a temperature greater than absolute zero. It is among three fundamental modes of heat transfer and represents a conversion of thermal energy into electromagnetic energy. Examples of thermal radiation include visible light emission and infrared light radiation. Based on the electromagnetic spectrum, IRR is invisible infrared light emission with wavelength higher than that of visible light, and lower than that of microwaves ranging from approximately $760 \mathrm{~nm}$ to $1000 \mu \mathrm{m}$. Moreover, physical parameters of infrared radiation may be occured the energy jump of molecule oscillation or rotation. In general, objects emit IRR across a spectrum of wavelengths. 
In thermal radiation theory, Planck's law is the basic regulation for black body radiation. Planck's law theoretically illuminates the relationship among spectral radiance, wavelength and thermodynamic temperature of black body. Planck's law was given as (Planck 1959):

$$
\mathrm{B}_{\lambda}(\lambda, \mathrm{T})=\frac{2 h c^{2}}{\lambda^{5}} \cdot \frac{1}{\mathrm{e}^{c h / \lambda k T-1}}
$$

where $\mathrm{B}_{\lambda}$ is the spectral radiance, $\mathrm{W} \mathrm{m}^{-2} \mu \mathrm{m}^{-1} ; c$ is the speed of light, $\mathrm{m} \mathrm{s}^{-1} ; h$ is Planck's constant, $\mathrm{J} s ; \lambda$ is the wavelength, $\mu \mathrm{m} ; \mathrm{T}$ is the absolute temperature of object, $\mathrm{K}$; And $k$ is the Stefan-Boltzmann constant, $\mathrm{J} \mathrm{m}^{-2} \mathrm{~K}^{-4}$.

Planck's law gives the spectral blackbody emissive power distribution in thermal equilibrium at different temperatures. The spectral radiance of a body indicates the amount of energy it gives off as radiation of different wavelengths. The Planck radiation has a maximum intensity at a specific wavelength that depends on the temperature. In addition, it is also revealed that the higher the temperature of a body, the more radiation it emits at every wavelength. Therefore, the radiant properties of a black body are only dependent on the absolute temperature and have nothing to do with the material composition.

In this chapter, the thermographic camera was applied in the passive mode without the use of any extra heat resources. Infrared thermography (IRT) could visualized the temperature changes over the viewed rock surface. When coal samples were loaded, the thermographic camera was used to observe and capture IRR variation during the whole loading process. In the IRR detection test, temperature data and thermograms of coal surface would be obtained. IRR emitted from coal surface was detected by the focal plane array (FPA) detector in the thermographic camera. The emitted infrared radiation was transformed into temperature by thermographic camera, so temperature reflected the radiant energy emitted from the object. By calibration, the relation between surface temperature and radiation could be established. The relationship between radiant energy and thermodynamic temperature of loaded coal samples was interpreted by the Stefan-Boltzmann law (Boltzmann 1884):

$$
\mathrm{M}=\varepsilon \sigma T^{4}
$$

where $\mathrm{M}$ is the radiant exitance, $\mathrm{W} \mathrm{m}^{-2} ; \varepsilon$ is the emissivity of object, $0<\varepsilon<1 ; \sigma$ is the Stefan-Boltzmann constant, $\mathrm{J} \mathrm{m}^{-2} \mathrm{~K}^{-4}$; and $\mathrm{T}$ is the absolute temperature of object, $\mathrm{K}$.

Stefan-Boltzmann law described the radiation emitted from an object in its absolute temperature. Meanwhile, Stefan-Boltzmann law stated the total energy radiated per unit surface area of an object across all wavelengths per unit time. Given that the tested samples were small-scale sized and coal surface was smooth, we assumed that the emissivity values were constant for wavelengths emitted from the surface. In other words, coal sample was approximatively regarded as a grey body. According to Stefan-Boltzmann law, the radiation intensity of loaded 
specimen was biquadratic to its absolute temperature. Temperature was an important indicator of IRR intensity and could reflect the radiant energy variation quantitatively. Therefore, temperature on coal surface was used to illustrate an index reflecting the thermal state and the complicated physico-mechanic process inside the loaded coal samples (Wu et al. 2006a, b).

Regarding the thermogram on coal surface, it is composed of a matrix of pixels whose temperature varies with stress during the loading process. Moreover, the temperature of each pixel at different locations may differ significantly. Therefore, the mean temperature of coal surface was introduced to describe the overall IRR variation of loaded coal samples during the deformation process. The mean temperature for each thermogram of the same coal sample could be calculated and analysed by the thermographic camera. The mean temperature was a general reflection of surface IRR energy of loaded rock, and could be selected as the integral index to study the temporal evolution of IRR (Wu et al. 2006a, b). In the experiment, the thermograms were visually shown on the display screen of thermographic camera, and the temperature data were easily obtained and stored with the use of IRT technique.

\subsubsection{IRT Characteristics of Coal Samples}

Temperature changed on the overall surface of coal samples were recorded during the process of uniaxial compression. To facilitate description of experimental results of tested coal samples, author chose representative sample M4 as an example. Figure 7.1 showed the relationship between stress-strain curve of limestone sample and mean temperature on the coal surface during the entire loading process. The deformation and fracturing processes of loaded coal samples had four stages, including the original crack compaction stage, the elastic deformation stage, the plastic deformation stage and the post-peak failure stage. As shown in Fig. 7.3, IRR was observed and detected during the entire loading process. The mean temperature on the sample surface varied with the progress of loading and had stage-changed characteristics:

(1) Initial loading and frist cycle phase: This stage was approximately $20 \%$ of the entire loading process. The concaved upwards curve during this stage indicated the closure of original microstructure under the uniaxial compression. As shown in Fig. 7.3, the average temperature rises at the beginning of loading. Subsequently, it changed from rise to down, and then rase again. It fluctuated significantly at early stage, with the maximum temperature fluctuating by $1.45{ }^{\circ} \mathrm{C}$. The reason might be due to the internal microfracture closure of coal sample.

(2) The second cycle phase and the phase before failure: This stage was approximately $40 \%$ of the entire loading process. At the end of the second cycle stage, 


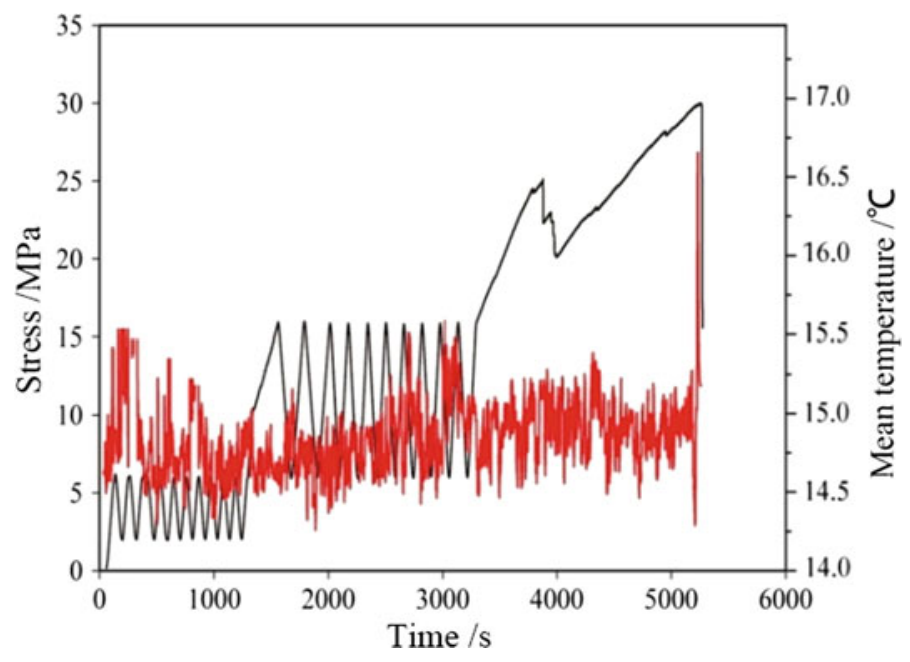

Fig. 7.3 Relationship between the stress-strain curve and the mean temperature

the average maximum temperature was appeared. With the loading into the circulation in the third phase (the phase before failure), average temperature started to down, and then into the relatively stable fluctuation period until to coal failure. In the period of fluctuation in about $4300 \mathrm{~s}$, there was a local maximum values of about $15.3{ }^{\circ} \mathrm{C}$. This was consistent with the dilatancy of coal sample during loading. With the increase of load, micro-cracks initiated, propagated, and merged progressively, the volume of coal samples began to swell, and the average infrared radiation temperature fluctuation regularity.

(3) The failure stage: In this stage, the stress dropped sharply to zero, and sample rupture occurred. Accordingly, the mean temperature decreased quickly after the peak stress.

As shown in Fig. 7.4, thermal radiation was detected and obtained in the entire loading process. The mean temperature of sample surface changed with time and period feature. Infrared radiation temperature percentage change had good regularity during the whole loading process of coal sample. In the compaction stage and elastic stage, the range of infrared radiation temperature distribution was relatively wide. In the plastic stage and failure time, the average temperature was high, especially the moment of destruction, average infrared radiation temperature of the maximum percentage was up to $16.3{ }^{\circ} \mathrm{C}$, as shown in $\mathrm{F}$ point in Fig. 7.4 , the average temperature increased approximately $2{ }^{\circ} \mathrm{C}$.

Analyzing the temperature variation features in different regions on rock sample surface, three different regions, region $\mathrm{A}$, region $\mathrm{B}$ and region $\mathrm{C}$, were delineated on the thermogram, as shown in Fig. 7.5. It was noted that region $\mathrm{B}$ and region $\mathrm{C}$ in 


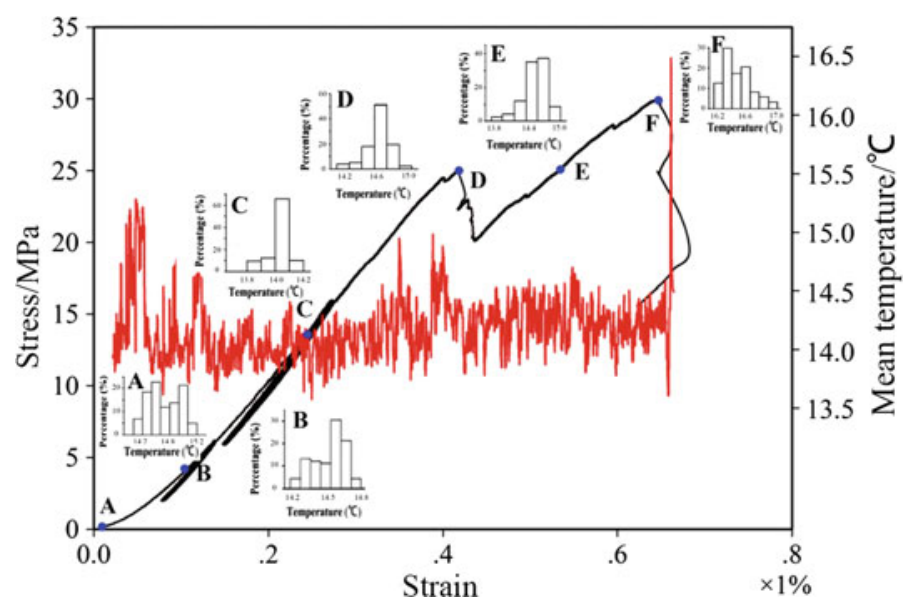

Fig. 7.4 Relationship between the stress-strain curve and the mean temperature

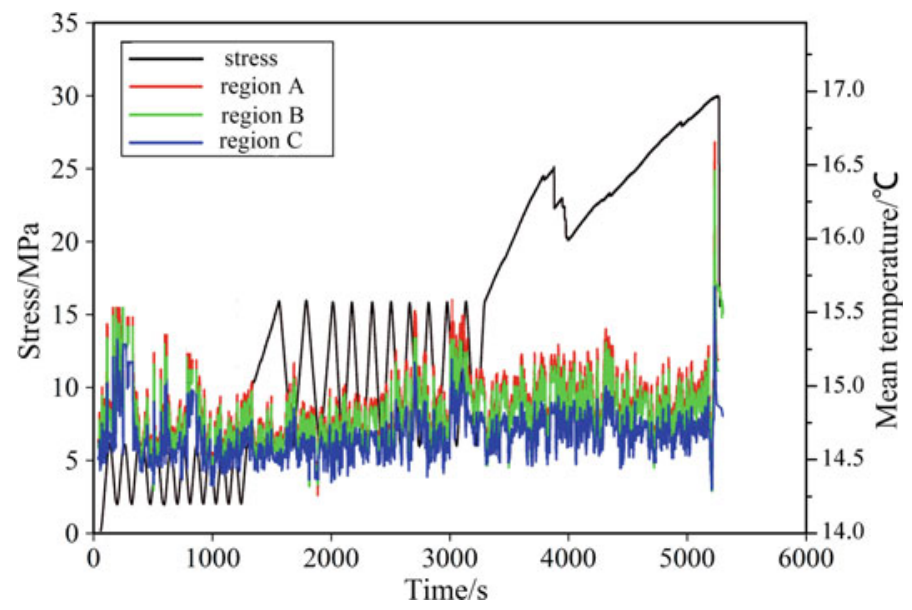

Fig. 7.5 Mean temperature variations in the three different regions

Fig. 7.5 were the fracturing regions at failure. Figure 7.6 showed the evolution of mean temperature in different regions in the entire loading process.

Analyzing the temperature variation features in different regions on rock sample surface, three different regions, region $\mathrm{A}$, region $\mathrm{B}$ and region $\mathrm{C}$, were delineated on the thermogram. As shown in Figs. 7.5 and 7.6, the evolution of mean temperature was showed in different regions in the entire loading process.

As shown in Fig. 7.6, variation trend of the mean temperature in three different regions was consistent on the whole sample. The mean temperature of region B was 
Fig. 7.6 Three different delineated regions in the thermogram

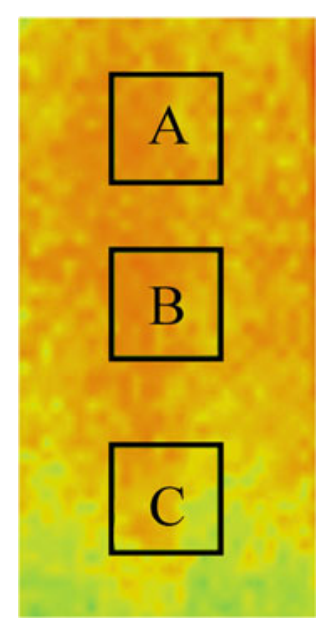

slightly lower than that in region $\mathrm{A}$, and much higher than that in region $\mathrm{C}$ during rock deformation period. It was pointed out that mean temperature of overall rock surface was between the mean temperature in region $\mathrm{A}$ and the mean temperature in region $\mathrm{B}$, indicating that region $\mathrm{C}$ was the low-temperature IRR region, whereas region $\mathrm{A}$ and region $\mathrm{B}$ were the high-temperature regions.

An abnormal precursory phenomenon of "rising-dropping-rising" in the average temperature appeared at the end of loading process. Especially, at the time about $4300 \mathrm{~s}$, average temperature change of regional $\mathrm{C}$ was not large while the average temperature increased significantly in the region $\mathrm{A}$ and $\mathrm{B}$. In addition, when the load was at peak stress, the average temperature of region A was about $16.6{ }^{\circ} \mathrm{C}$, region $\mathrm{B}$ was about $16.47{ }^{\circ} \mathrm{C}$, and region $\mathrm{C}$ was about $15.65^{\circ} \mathrm{C}$. That is to say, the average temperature of region $\mathrm{A}$ and region $\mathrm{B}$ was close, while region $\mathrm{C}$ was much lower than that of A and B, which indicated that the main inside cracks of sample was located in the upper part of sample, where a large number of infrared heat radiation were released. This phenomenon could help locate the damage of coal sample.

Given that the initial mean temperatures in the three regions were different, the mean temperature variation ranges at the same time were not shown. Therefore, investigating the mean temperature variation ranges in different regions during the loading process, we defined the difference between the mean temperature at a certain loading time and the initial mean temperature at the initiation of loading as the mean temperature increment. As shown in Fig. 7.7, the mean temperature increment of three areas of coal sample was shown during the whole loading process, the difference increment of the average temperature between the three regions during the initial loading phase was relatively low and the curve 


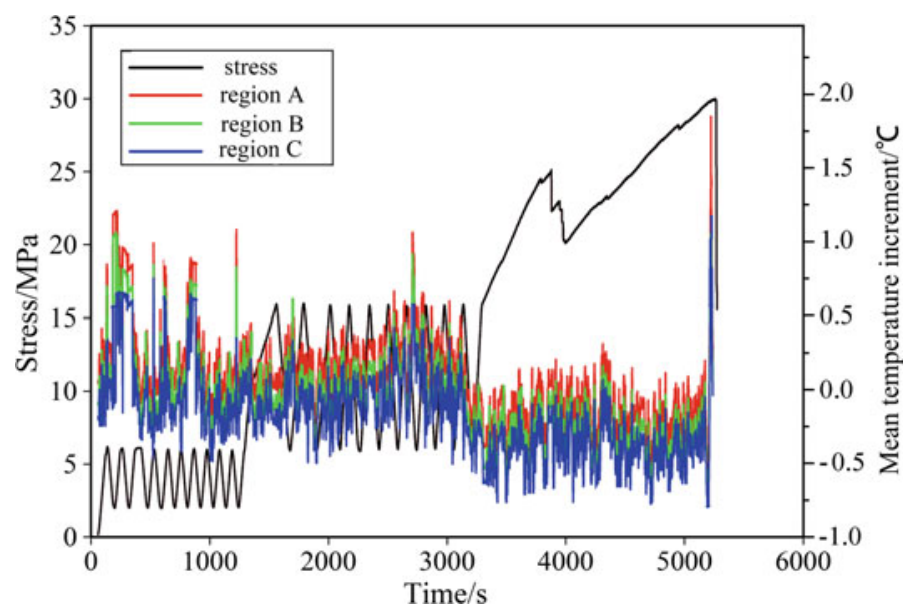

Fig. 7.7 Mean temperature increment variations in the three different regions

coincidence was relatively high. However, the average temperature increment of three different regions, with the persistence of loading, especially after $1300 \mathrm{~s}$, began to show different changes, and the coincidence degree of the average temperature increment curve in three different regions decreased. As shown in Fig. 7.7, the average temperature increment of region A was slightly higher than region B, which was higher than region $\mathrm{C}$. All three different regions showed an anomalous precursory phenomenon of "rising-dropping-rising" before the peak pressure in the average temperature increment. As for the average temperature increment, the fluctuations of temperature increment in three regional were comparatively evident at the beginning of $1000 \mathrm{~s}$. From 1500 to $2800 \mathrm{~s}$, the average temperature increment in three regions increased steadily and the temperature increment increased by nearly $1{ }^{\circ} \mathrm{C}$. This $1{ }^{\circ} \mathrm{C}$ was obtained at about $2800 \mathrm{~s}$ in both region $\mathrm{A}$ and $\mathrm{B}$, while the average temperature increment of $\mathrm{C}$ was about $0.5^{\circ} \mathrm{C}$. After then, the average temperature increment began to decrease, the average temperature increment of region $\mathrm{A}$ and $\mathrm{B}$ drops to $-0.5{ }^{\circ} \mathrm{C}$ and began to change smoothly, where the number of $\mathrm{C}$ was $-0.75{ }^{\circ} \mathrm{C}$. When stress of coal sample reached the peak value, the average temperature increment of region $\mathrm{A}, \mathrm{B}$ and $\mathrm{C}$ were $1.8,1.5$ and $1.2{ }^{\circ} \mathrm{C}$, respectively. It was proved that the average temperature increment of region $\mathrm{A}$ and $\mathrm{B}$ was significantly higher than region C. At the peak pressure, coal sample had a major rupture in region $\mathrm{A}$, which showed obvious spots and streaks in the infrared thermal image, which indicated that monitoring captured higher radiation temperatures and extremely dense radiation appearances. After the loading peak, the average temperature increment of three regions decreased instantaneously when pressure value suddenly decreased, and coal sample was finally failure. 


\subsubsection{IRT Prediction for Rock Failure}

The mean temperature on the coal surface, as a quantitative index, was used to characterize the evolution of temperature field during the entire loading process. As stated previously, the mean temperature abnormality of "rising-dropping-rising" in the later loading period was considered as the temperature curve precursor for rock failure. Accordingly, the abnormal precursors of obvious high temperature bright spots or bright bands in the infrared thermal image showed that a large amount of infrared radiation energy was released, and coal sample would be failure. Combined with the anomalies of average temperature increment curve and the anomaly of infrared thermal image, the infrared anomaly precursors could be taken as the infrared precursors of coal samples failure. Therefore, the mean temperature increment interval of "dropping-rising" could be used as a predicting point for coal failure (as shown in Fig. 7.8).

The abnormal precursors on thermograms and on the mean temperature curve occurred synchronously before coal failure. Thus, the mean temperature and thermograms should be analysed comparatively, and it would be more accurate and effective for identifying the infrared precursor of rock failure or mine hazards. On the one hand, the mean temperature was used as a quantitative index to identify the abnormal precursor points, including the predicting point and the key predicting point, which presented the potential collapse time. On the other hand, the spatiotemporal evolution of thermograms could reflect the temperature distribution with time, and the spatial location of abnormal precursor on the thermogram might help predict the anticipated collapse area. Based on the integrated investigation on the mean temperature precursor and the thermogram precursor, it was easier to

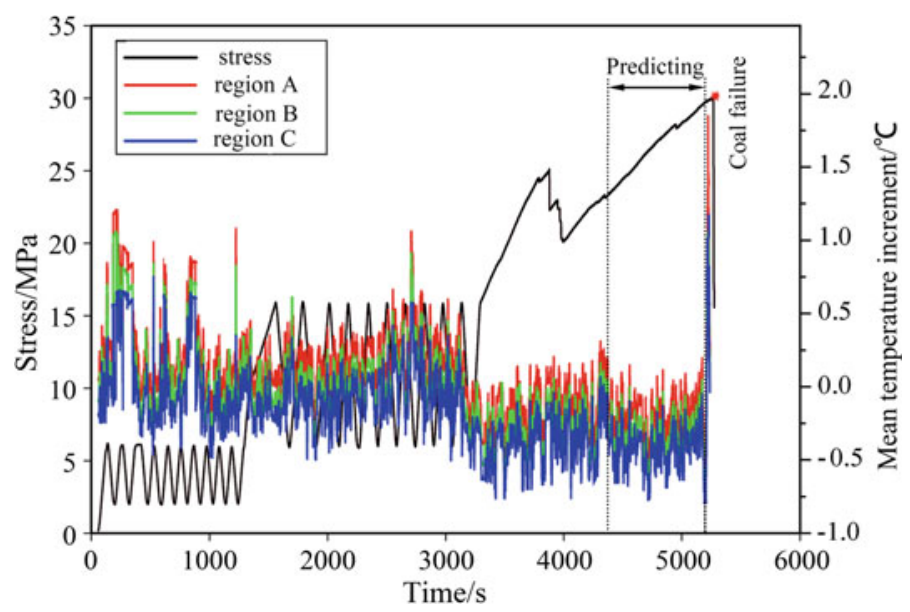

Fig. 7.8 Prediction of coal failure by mean infrared radiation temperature increment 
predict potential hazards and obtain its potential occurrence time and location. Therefore, effective measures could be made timely to prevent losses of personnel and equipment.

\subsection{Experimental Investigation on Predicting Rock Failure Using Load/Unload Energy Response Ratio Theory}

\subsubsection{LURR Characteristics of Coal Samples}

Based on the experiment of Sect. 7.2, AE energy was collected to calculate the total amount of AE energy, the summation for AE energy release square root and LURR $\left(Y_{1}\right.$ and $\left.Y_{2}\right)$. The calculated results of coal specimen M1 in the second stage were schematically shown in Table 7.2. Based on LURR theory, the loading response

Table 7.2 AE energy results collected and analysed from the loading process of coal sample M1

\begin{tabular}{|c|c|c|c|c|c|c|c|}
\hline No. & $\begin{array}{l}\text { Loading } \\
\text { way }\end{array}$ & $\begin{array}{l}\text { Start } \\
\text { time }\end{array}$ & $\begin{array}{l}\text { End } \\
\text { time }\end{array}$ & $\begin{array}{l}\text { AE energy } \\
\text { summation/ } \\
\text { J }\end{array}$ & $\begin{array}{l}\text { LURR } \\
\left(Y_{1}\right)\end{array}$ & $\begin{array}{l}\text { Summation for AE } \\
\text { energy release square } \\
\text { root// } \mathrm{J}^{1 / 2}\end{array}$ & $\begin{array}{l}\text { Benioff } \\
\text { strain } \\
\left(Y_{2}\right)\end{array}$ \\
\hline \multirow[t]{2}{*}{1} & Loading & 877 & 917 & 109,861 & \multirow[t]{2}{*}{5.13034} & $16,794.65$ & \multirow[t]{2}{*}{6.73718} \\
\hline & Unloading & 918 & 957 & 21,414 & & 2492.83 & \\
\hline \multirow[t]{2}{*}{2} & Loading & 958 & 997 & 23,727 & \multirow[t]{2}{*}{3.44569} & 2903.99 & \multirow[t]{2}{*}{2.72167} \\
\hline & Unloading & 998 & 1037 & 6886 & & 1066.99 & \\
\hline \multirow[t]{2}{*}{3} & Loading & 1038 & 1077 & 9810 & \multirow[t]{2}{*}{2.56874} & 1386.53 & \multirow[t]{2}{*}{1.91936} \\
\hline & Unloading & 1078 & 1118 & 3819 & & 722.39 & \\
\hline \multirow[t]{2}{*}{4} & Loading & 1119 & 1158 & 5265 & \multirow[t]{2}{*}{1.11476} & 879.96 & \multirow[t]{2}{*}{1.23550} \\
\hline & Unloading & 1159 & 1198 & 4723 & & 712.23 & \\
\hline \multirow[t]{2}{*}{5} & Loading & 1199 & 1238 & 5070 & \multirow[t]{2}{*}{1.61722} & 672.63 & \multirow[t]{2}{*}{1.42903} \\
\hline & Unloading & 1239 & 1278 & 3135 & & 470.69 & \\
\hline \multirow[t]{2}{*}{6} & Loading & 1279 & 1319 & 2195 & \multirow[t]{2}{*}{0.82210} & 384.52 & \multirow[t]{2}{*}{1.18179} \\
\hline & Unloading & 1320 & 1359 & 2670 & & 325.37 & \\
\hline \multirow[t]{2}{*}{7} & Loading & 1360 & 1399 & 2695 & \multirow[t]{2}{*}{1.07200} & 414.92 & \multirow[t]{2}{*}{1.13094} \\
\hline & Unloading & 1400 & 1439 & 2514 & & 366.88 & \\
\hline \multirow[t]{2}{*}{8} & Loading & 1440 & 1479 & 3422 & \multirow[t]{2}{*}{1.33411} & 408.43 & \multirow[t]{2}{*}{1.05149} \\
\hline & Unloading & 1480 & 1520 & 2565 & & 388.43 & \\
\hline \multirow[t]{2}{*}{9} & Loading & 1521 & 1560 & 1898 & \multirow[t]{2}{*}{1.23007} & 363.37 & \multirow[t]{2}{*}{1.01953} \\
\hline & Unloading & 1561 & 1600 & 1543 & & 356.41 & \\
\hline \multirow[t]{2}{*}{10} & Loading & 1601 & 1640 & 1689 & \multirow[t]{2}{*}{0.99004} & 221.16 & \multirow[t]{2}{*}{0.97548} \\
\hline & Unloading & 1641 & 1680 & 1706 & & 226.72 & \\
\hline
\end{tabular}

Note Only the testing results from coal specimen M1 were included in Table 7.2, in consideration of mass data from all the five coal specimens 
rate of coal specimens was equal to the unloading rate, or $X_{+}=X_{-}$in the first stage. In addition, research on predicting rockburst was principally focused on the plastic deformation stage. Little attention was paid on AE energy in this chapter.

The variation characteristics associated with coal specimen failure could be obtained based on LURR theory and AE energy. In Table 7.2, the total AE energy was observed for an abnormal phenomenon during No. 1 step. Additionally, the summation for AE energy release square root was also observed for abnormal phenomenon during No. 1 step. These phenomenons played a meaningful role in predicting rockbursts.

In the laboratory experiment, AE energy expressed the variations in the different stress states. Figure 7.9 shows AE energy variation process of all coal specimens. The mechanical behaviour of coal specimens could be determined by the original cracks compaction and elastic deformation. During the loading process, the original cracks in coal specimen were gradually closed. Due to the heterogeneity of coal specimens, the internal partial region of coal specimens quickly produced stress concentration. Thus, AE energy was increased obviously. Strong AE energy signals were mainly a result of coal grain sliding friction and internal micro-crack closure. However, during the first stage of load/unload process, AE signals were mainly caused by coal grain sliding friction, which resulted in a small amount of AE energy release.

In the second stage shown in Table 7.2, the stable-unstable development of micro-cracks and elasto-plasticity deformation were analytical gained.
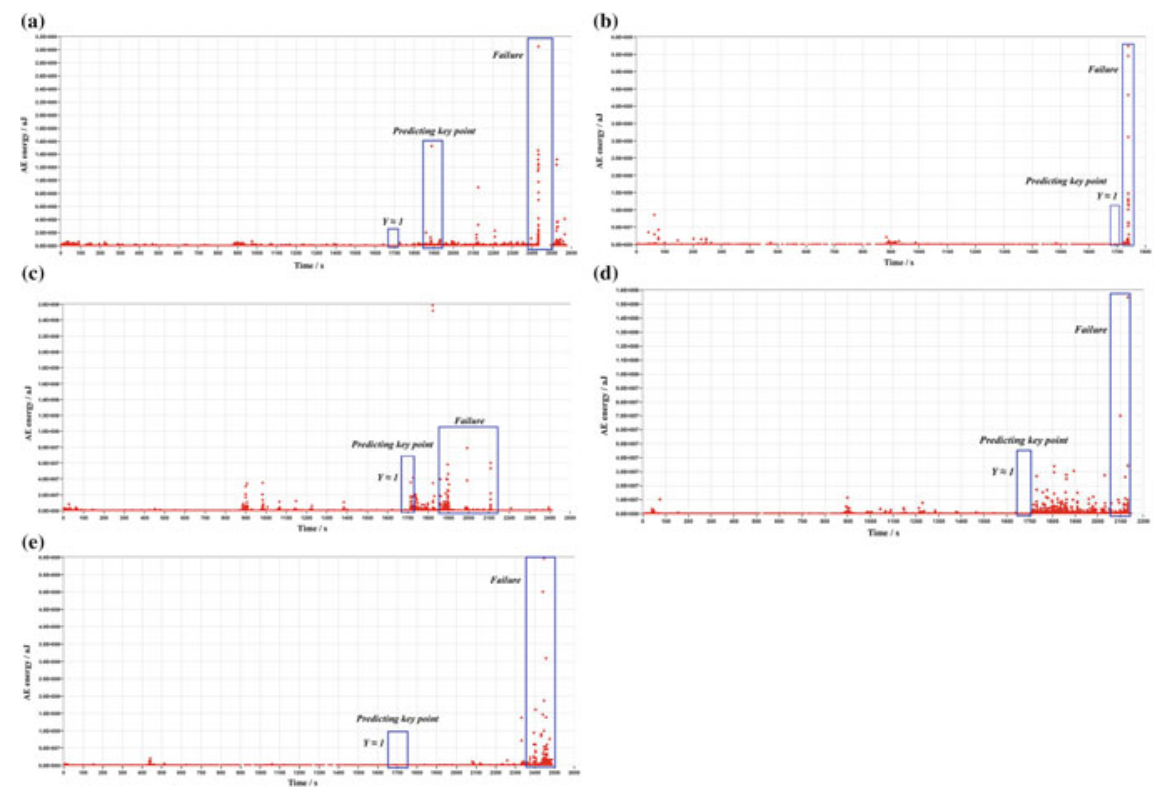

Fig. 7.9 AE energy release of coal specimens 
Developmental variations in the micro-cracks and failures of coal specimens were analysed. These fractures could be recovered due to stress concentration, strain energy release and AE energy release. During the load/unload process, the extension of internal natural micro-cracks and formation of new micro-cracks lead to a weakening in the mechanical properties (such as compressive strength and void ratio) of coal specimens.

As shown in Fig. 7.9, AE energy was released during the first stage less than that the second and third stage. During the second stage, the release of AE energy was apparent, indicating that new cracks were generated in coal specimens and propagated rapidly. When coal specimens were loaded into the third section, the intensive AE energy release of coal specimens was more obvious. A great deal of AE energy release was mainly concentrated before coal failure. Then, AE energy release of coal specimens decreased rapidly. This phenomenon could be used as the precursor information of coal failure. Similar conclusions related to predicting key points were obtained using AE/MS monitoring, AE energy and LURR theory. However, as shown in Fig. 7.9a, AE energy of coal specimen M1 was suddenly concentrated released in a period of time before coal specimens' failure. This phenomenon could be due to the difference of brittleness and heterogeneity of five coal specimens.

$\mathrm{AE}$ energy data were obtained to analysis in the experiments. According to LURR equation, AE energy data were processed and analysed using two methods. The total AE energy release in the second stage was proposed as a response rate $(X)$ in the first method. In the other way, the response rate $(X)$ was denoted by the summation for AE energy release square root in the second stage. This study focused on precursor information of rockburst during the plastic deformation stage.

As a result of suddenly AE energy concentrated release before coal failure, the mechanism of rockburst could be revealed using energy analysis. Many results were obtained by scholars; For example, the total energy release was considerably higher under dry state than saturated state, which is the same as the shallow bedding orientation (Wasantha et al. 2014). Energy propagation was determined by elastic strain energy (Rudnicki and Sternlof 2005). Therefore, the release of AE energy was used as a parameter to examine the prediction of rockburst in this study. As shown in Table 7.2 and Fig. 7.9, AE energy release in the first step was extremely larger than that in the other steps. Then, AE energy release began to reduce continuously. Based on the data collected in the test, many parameters change characteristics were obtained, such as the total AE energy, LURR $\left(Y_{1}\right)$, the summation for AE energy release square root and Benioff strain $\left(Y_{2}\right)$. From the first step to the third step of sample M1 in Table 7.2, AE energy in the loading step was obviously higher than that in the unloading step, and LURR value $\left(Y_{1}\right)$ was larger than 1 . During the sixth step, AE energy in the loading step was clearly less than in the unloading step, and LURR value $\left(Y_{1}\right)$ was less than 1 . During the seventh to tenth steps, AE energy in the loading step was closest to that in the unloading step, and LURR value $\left(Y_{1}\right)$ was close to 1 . However, based on the summation of AE energy release square root from the first step to fifth step, the value of summation for $\mathrm{AE}$ energy release square root during loading was greater than that during unloading, 


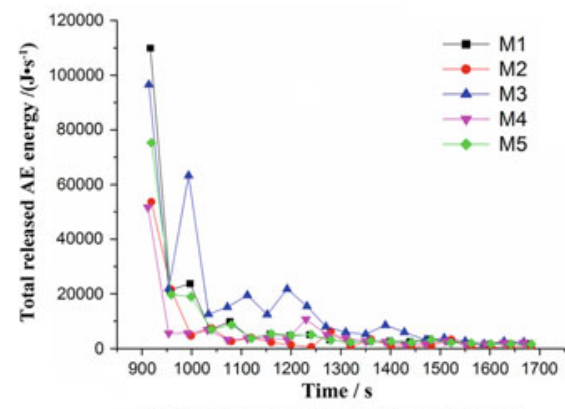

(a) Total variation in the released AE energy.

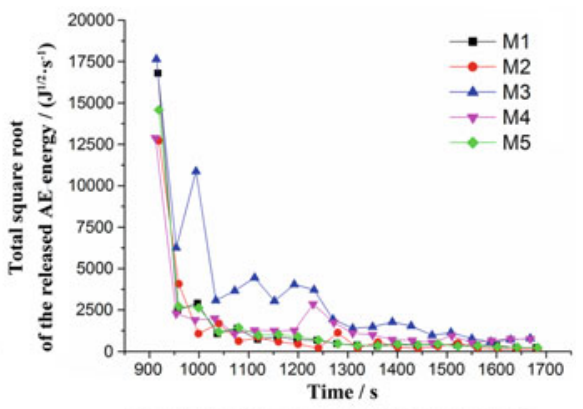

(b) Total Square root of the released AE energy.

Fig. 7.10 Variations of AE energy released of coal specimens under uniaxial load/unload condition

and Benioff strain $\left(Y_{2}\right)$ was larger than 1. During the 6-th step to 10-th step, the value of summation for AE energy release square root in the loading step was closest to that in the unloading step, and Benioff strain $\left(Y_{2}\right)$ was close to 1 . This phenomenon illustrated that more AE energy was released during loading than unloading. The micro-cracks in the coal samples were compacted in the first three loading steps.

As shown in Fig. 7.10, the variation characteristics of AE energy were highly consistent with the summation of AE energy release square root for specimens. These two parameters had similar change processes, as the initial released energy was larger than that after the sharp decrease. Meanwhile, the variation tendency of these two parameters became to a steady change after the sharp decrease. The internal micro-crack growth in the sample was mainly consistent with the energy release. The released AE energy variation could infer that the concentrated release of AE energy occurred at the beginning of the second stage. Then, AE energy accumulated before coal failure. Consequently, AE energy accumulation in plastic deformation stage could be used as precursor for rockburst.

\subsubsection{LURR Prediction of Coal Failure}

In this chapter, LURR $\left(Y_{1}\right)$ and Benioff strain $\left(Y_{2}\right)$ were used to analyse coal failure based on AE energy data and the relationship in Eq. (3.5). Figure 7.11 shows the variation characteristics of LURR $\left(Y_{1}\right)$ and Benioff strain $\left(Y_{2}\right)$. The trends of $Y_{1}$ and $Y_{2}$ values were highly similar and could be used to predict coal failure. $Y_{1}$ and $Y_{2}$ values were both larger at the beginning of the second stage. Then, they fluctuated around 1. Finally, both $Y_{1}$ and $Y_{2}$ values were close to 1 at the end of the second stage. The initial deformation and degree of damage of coal specimens could be determined based on the trends in $Y_{1}$ and $Y_{2}$ values. 


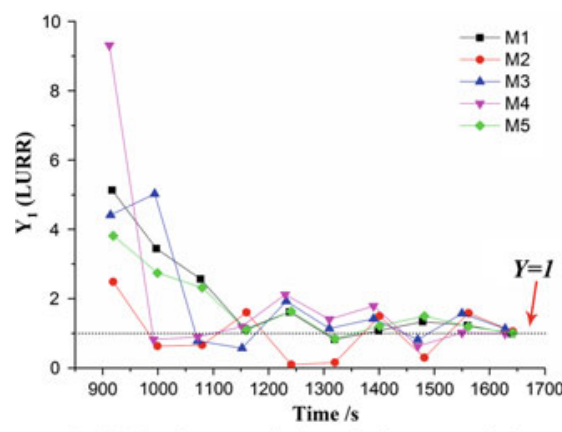

(a) $Y$, value variational characteristics.

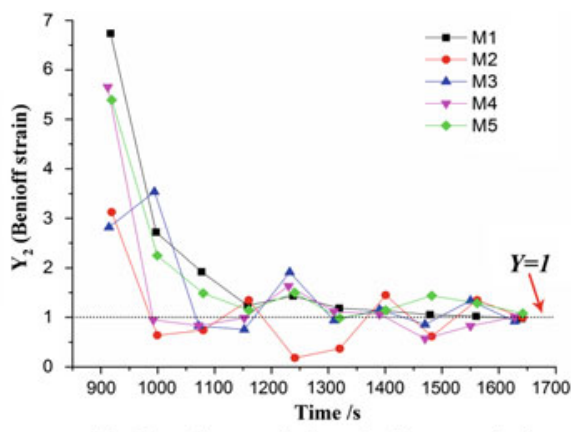

(b) $Y_{2}$ value variational characteristics.

Fig. 7.11 Variation characteristics of LURR $(Y 1)$ and Benioff strain $(Y 2)$ of coal specimens

As shown in Figs. 7.10 and 7.11, the fluctuation of $Y_{1}$ and $Y_{2}$ values was consistent with the AE energy accumulation on the time series. Therefore, LURR variation trend (the $Y_{1}$ and $Y_{2}$ values were both close to 1) could be viewed as precursor information for rockburst. The time points, where $Y_{1}$ and $Y_{2}$ values, were both close to 1 , they could be used as the predicting key point of coal failure. This point could be measured before coal failure. What is more, after this time point, coal specimens destroy completely in a short time. Thus, LURR theory, which combined with AE energy under uniaxial cyclic load/unload conditions, could be used to predict rockburst. Inevitably, this method still has shortcomings. Affected by the constitutive properties and loading speed of coal specimens, the time between the predicting key point and the time point of coal failure was very difficult to determine, which still needed more researches in the future.

\subsection{Experimental Investigation on Predicting Points Using Tangent Damage Factor for Rock Failure}

\subsubsection{Laboratory Test of Rock Failure}

In the experiment, the limestone specimens were taken from coal roof of Xie Zhuang coal mine in Shandong province. The mining depth is $1212 \mathrm{~m}$. With the depth of mining operation, mine disasters have occurred more easily than ever before. The limestone specimens were processed in the form of wet processing method in the laboratory. All samples were prepared in accordance with International Society for Rock Mechanics (ISRM) suggested shape and size and were processed into cylinders measuring approximately $50 \mathrm{~mm}$ in diameter and $100 \mathrm{~mm}$ in height, as shown in Table 7.3. Finally, six samples were obtained. Meanwhile, both the specimens' ends were polished when processing them and the unevenness of surface was controlled less than $0.05 \mathrm{~mm}$. Both the specimens' ends 
Table 7.3 The physical-mechanical parameters of 6 limestone samples

\begin{tabular}{l|l|l|l|l}
\hline Specimen ID & Height $(\mathrm{mm})$ & Diameter $(\mathrm{mm})$ & Density $\left(\mathrm{g} \mathrm{cm}^{-3}\right)$ & UCS $(\mathrm{MPa})$ \\
\hline $\mathrm{X} 1$ & 98.54 & 49.52 & 2.61 & 131 \\
\hline $\mathrm{X} 2$ & 102.12 & 51.20 & 2.85 & 132 \\
\hline $\mathrm{X} 3$ & 99.42 & 48.70 & 2.68 & 108 \\
\hline X4 & 97.84 & 48.30 & 2.64 & 103 \\
\hline X5 & 101.2 & 50.50 & 2.71 & 131 \\
\hline X6 & 99.28 & 48.64 & 2.66 & 130 \\
\hline
\end{tabular}

were perpendicular to the axis and the tolerance of angle was less than $0.25^{\circ}$. After preparing the early work, these specimens were loaded under uniaxial compression. Meanwhile, AE instrument was used to monitor.

This test adopted the axial displacement loading way and the loading speed was controlled at $0.005 \mathrm{~mm} / \mathrm{s}$. It is necessary to ensure that the loading machine and $\mathrm{AE}$ motoring system synchronously obtain the mechanical behaviors and AE characteristics under loading in real time.

The preamplifiers of $\mathrm{AE}$ were set at $40 \mathrm{~dB}$. AE testing system included 6 sensors for realizing real-time 3-D positioning of limestone samples during the entire deformation and fracture processes. Meanwhile, to reduce the influence of environment noise, the threshold value of PCI was set at $45 \mathrm{~dB}$ and the sampling rate was set at $1 \mathrm{MHz}$. To reduce the influence of end-effects, $6 \mathrm{AE}$ sensors were fitted on the limestone specimen $20 \mathrm{~mm}$ away from the top and bottom of specimens. Petroleum jelly and an adhesive band were used to fix the sensors on the specimen surface. To ensure that each channel normally collected signals, it was necessary to conduct the lead-break experiment near the $6 \mathrm{AE}$ sensors to verify that whether a good coupled relationship between sensors and the specimen.

\subsubsection{Define the TDF}

The nonlinear fracture and instability process of rock could be well described by the damage evolvement process. Therefore, in this chapter, the damage curves were used to analyze the fracture and instability process of limestone. Tangent damage factor (TDF) curves were used to predict rock failure. The damage evolvement process was regarded as the nonequilibrium statistical process. So the damage factor (DF) of rock follows statistical distribution. The statistical distribution of Weibull two-parameters was as follows: 


$$
\phi(F)=\frac{m}{F_{0}}\left(\frac{F}{F_{0}}\right)^{m-1} \exp \left[-\left(\frac{F}{F_{0}}\right)^{m}\right]
$$

where $F$ is the random distributed variable; $\phi(F)$ is the measure of micro-units damage; $m$ and $F_{0}$ are the distributed parameters, $m$ is parameter reacted the level of rock brittleness, $F_{0}$ is the macro average strength of rock.

DF reflects the damage degree of materials. Meanwhile, the damage degree is related to each defective micro-unit. These defects directly affect micro-units strength. The relationship between DF and the probability density of micro-units failure is as follows:

$$
\frac{d D}{d F}=\phi(F)
$$

Therefore, DF can be calculated as:

$$
D=\int_{0}^{F} \phi(F) d F=1-\exp \left[-\left(\frac{F}{F_{0}}\right)^{m}\right]
$$

Essentially, AE activity is consistent with the statistic distributed regularity of material internal defects. Therefore, AE count could reflect the distribution of damage. If the accumulated $\mathrm{AE}$ count is $\Omega m$ (ringing counts or energy counts) on the whole cross section completely destroyed, AE accumulation count could be expressed during the deformation and fracture process, as follows:

$$
\Omega=\Omega_{m} \int_{0}^{F} \phi(x) d x
$$

When $\Phi(\mathrm{x})$ is taken as the function (7.4), the expression (7.6) can be calculated as:

$$
\frac{\Omega}{\Omega_{m}}=1-\exp \left[-\left(\frac{F}{F_{0}}\right)^{m}\right]
$$

Comparing expression (7.6) with (7.7), it can get the damage expression caused by loading:

$$
\frac{\Omega}{\Omega_{m}}=D
$$

Therefore, the damage which is defined by AE parameters can be calculated by expression (7.8). In other words, the damage can be expressed by accumulated ringing counts or accumulated energy counts. 
The change process of DF could be used to describe the process of rock failure and establish the power law relationship between damage and time. It provides important information to identify the predicting key points of rock failure. Therefore, it is important to establish the power law relationship between damage and time. The previous derive the expression of damage with the Weibull statistics distribution theory and the strength equivalent hypothesis theory is as follows:

$$
D=1-\exp \left[-\frac{1}{m}\left(\frac{\varepsilon}{\varepsilon_{0}}\right)^{m}\right]
$$

where $D$ is damage variable; $m=\frac{1}{\ln \left[\frac{\varepsilon_{0}}{\sigma_{0}}\right]}$ and $\varepsilon$ are the shape parameters of rock; $\varepsilon_{0}$ is the peak strain. So it is easy to infer that DF is associated with the strain of limestone sample, the initial elastic modulus, the peak strain and the peak stress.

In the test, the stain of limestone is proportional to the loading time under the loading condition. The scaling factor of expression is $\frac{v}{h}$. So the relationship between strain and time of limestone is as follows:

$$
\varepsilon=\frac{v t}{h}
$$

The power law relationship can be obtained from Eqs. (7.9) and (7.10):

$$
D=1-\exp \left[-\frac{v^{m}}{m h^{m} \varepsilon_{0}^{m}} t^{m}\right]
$$

From Eq. (7.11), DF is associated with the loading speed, the shape and size and the peak strain. At the same time, DF has a power law relationship with the loading time. Based on DF, an expression of TDF was established. Assume that incremental damage value was $\Delta D$ and the correspondingly changing time value was $\Delta t$. TDF was calculated according to the incremental damage value $\Delta D$ and the correspondingly changing time value $\Delta t$. As follow:

$$
k_{D}=\frac{\Delta D}{\Delta t}
$$

where $\Delta D=D_{i}-D_{i-1}, \Delta t=t_{i}-t_{i-1}$. Based on the damage curves, in this chapter, the proposed the concept of TDF which could identify the predicting key points of rock failure rapidly and exactly. At the same time, it was helpful to realize in time predicting in the process of rock failure and provide important predicting information for rockbursts and other hazards.

Under the effects of loading and environment, rockmass damage is caused by the original material's micro-cracks and micro-holes. During the loading process, $\mathrm{AE}$ events are generated from the interaction of internal micro-cracks, expansion, linking and macro-failure within these samples. It is related to the evolution and development of cracks in the internal rock. The changes of AE events count are 
consistent with the damage evolution patterns of samples. Therefore, theories of damage mechanics can be used to establish evolvement models based on the characteristic parameters of $\mathrm{AE}$ in the rock failure process.

In this chapter, $\mathrm{DF}$ was introduced with $\mathrm{AE}$ parameters; the power law relationship was obtained between damage and time. Then, TDF was defined by the tangent curves of DF; it could be described the process of rock failure and identify the predicted key points. The graded predicting method was proposed in the process of rock failure. Finally, a damage-time-failure-predicting model was established.

According to the change of $\mathrm{AE}$ events count, which were consistent with the damage evolution patterns of rock, the DF was easily obtained, being defined by $\mathrm{AE}$ counts. AE damage degree was defined as the measure point divided by the total counts.

\subsubsection{Damage Characteristic of Limestone Failure}

Based on AE parameters of 6 specimens in the process of rock failure under uniaxial compression, DF curves were obtained, as shown in Fig. 7.12.

The correlation coefficients of samples X1, X2 and X6 were 0.99838, 0.9834 and 0.99599 , respectively. DF curves were presented the characteristic of 'rapid growth-stable growth-sharp growth-stability', which can well describe the process of rock failure. They had a more obviously phased change, such as X2, X4 and X5.

Based on the experimental data of two groups and the fitted results, DF value has well relationship with the loaded-time. The relationship of them is as follows:

$$
D=A_{2}+\frac{A_{1}-A_{2}}{1+\left(t / x_{0}\right)^{p}}
$$

All the expressions of above fitted DF and loaded-time are shown in Table 7.4. As presented in the first group, $x_{0}$ is directly correlated with the loaded-time. $A_{2}$ is closer to the peak point of DF value, while $A_{1}$ is near 0 . The power law relationship between $D$ and the loaded-time is from 1 to 3 . The power law equation between DF and the loaded-time for the first group is as follows:

$$
D=\left\{\begin{array}{c}
1.23176-\frac{1.20616}{1+(t / 446)^{2.37198}} \\
1.77072-\frac{1.76614}{1+(t / 93.63)^{1.89744}} \\
1.61395-\frac{1.54716}{1+(t / 60.05)^{1.55592}}
\end{array}\right.
$$

where $A_{1}$ is $0,1<A_{2}<2,1<p<3 ; x_{0}$ is the relationship with the loaded-time $t$ in the process of rock failure. Therefore, a damage model applied to the limestone 


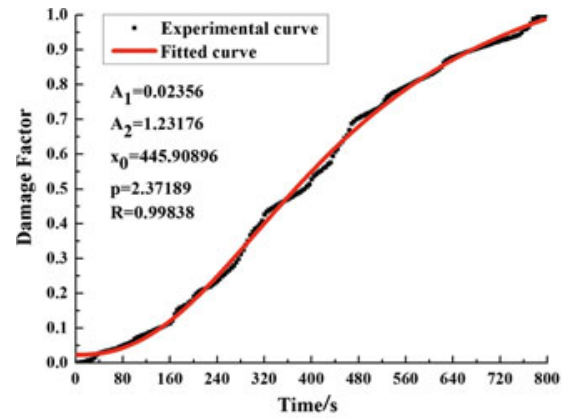

(a) $\mathrm{X} 1$

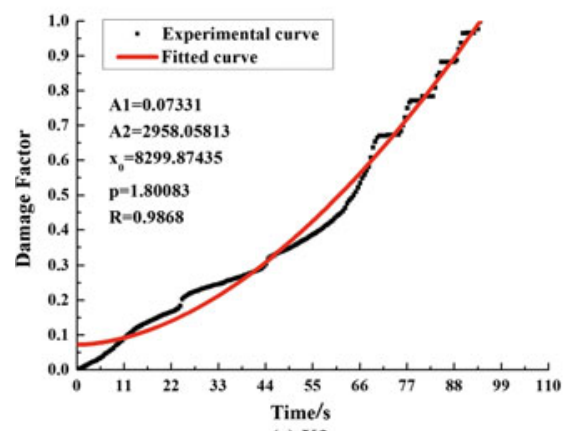

(c) $\mathrm{X} 3$

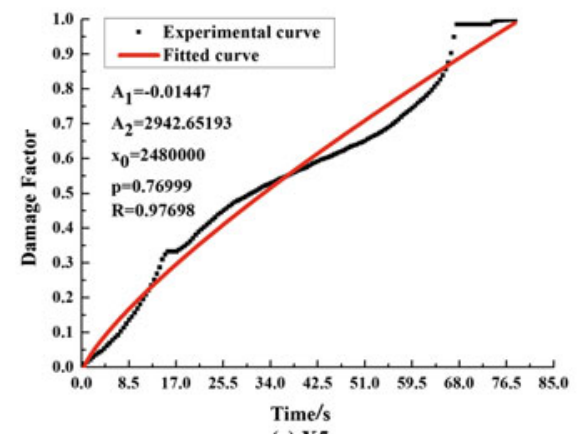

(e) X5

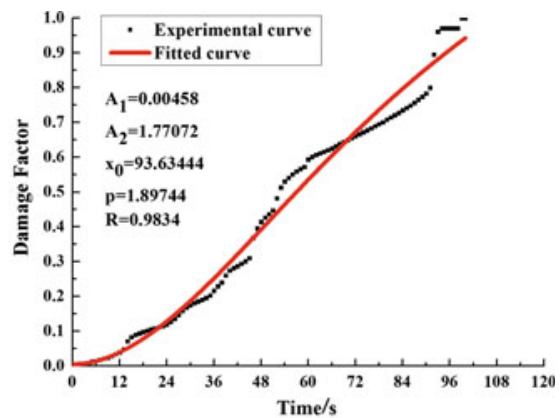

(b) $\mathrm{X} 2$

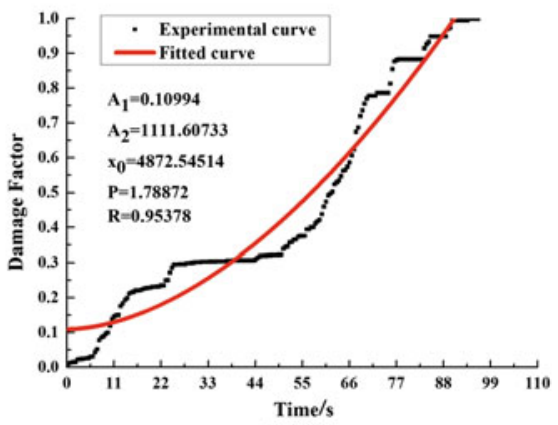

(d) $\mathrm{X} 4$

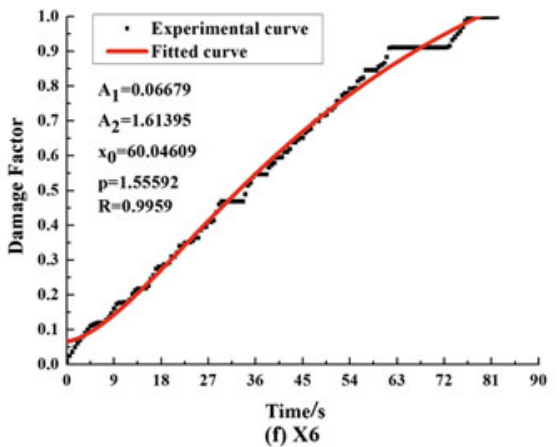

Fig. 7.12 The damage factor curves of samples

rock could be established. The loaded-time $t$ was viewed as a variable in the damage model, as follows:

$$
D=A_{2}\left[1-\frac{1}{1+\left(t / t_{0}\right)^{p}}\right]
$$

where $t_{0}$ is the loaded-time constant; $A_{2}$ is the material constant; $p$ is the power between DF and the loaded-time and is generally $1-3$. 


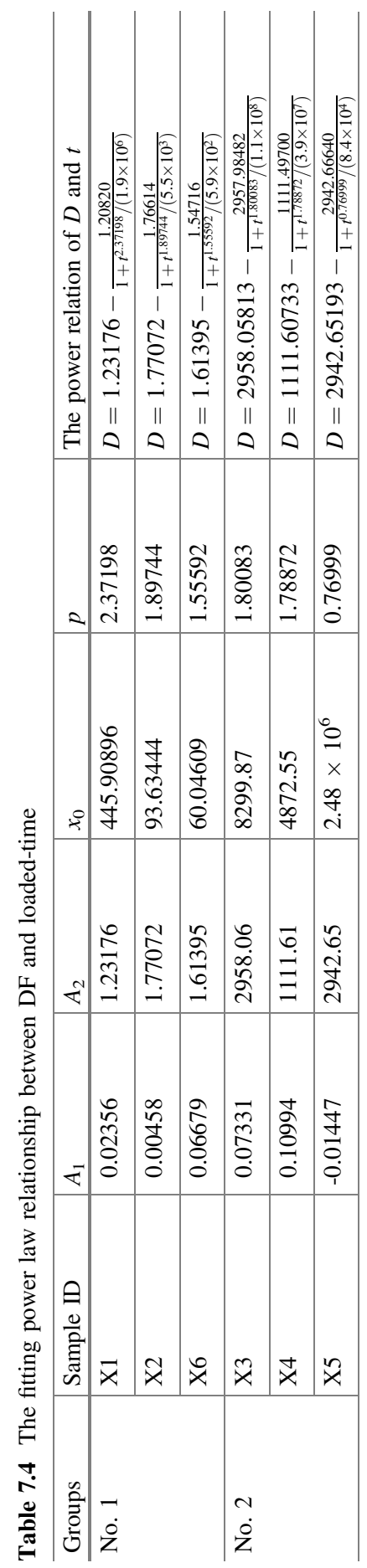


In this chapter, specimen $\mathrm{X} 5$ was selected to describe the experimental process of rock failure. TDF data is $\mathrm{k}_{\mathrm{B}}$ to 0 . The predicting data of sample X5 is as follows in Table 7.5 .

The damage curves have many phased grades, which could be used to describe the process of limestone failure. TDF curve is shown in Fig. 7.13.

Many ways could be used to define the DF, including the macroscopic perspectives and micro perspectives. In this chapter, DF was defined using AE counts. The proposed TDF was used to predict the rock failure. As shown in Fig. 7.12, DF curves and fitting curves of 6 limestone specimens were obtained. The fitting degree of samples showed the characteristic of phased with sharply rising in some phases. This phenomenon occured might because many natural joints existed in the limestone samples, which caused AE change. In the loaded process, the original joints were gradually closure, developed and merged, which caused AE ringing counts to increase sharply. DF curves of limestone samples presented the phased characteristic of rock failure.

As shown in Table 7.5, a power law relationship between DF and predicting time was presented; Predicting time could be inferred by using DF value. It could be used to estimate not only the nonlinear process of rock failure qualitatively but also the predicting time quickly. As shown in Fig. 7.13, the power law functions could be obtained based on the high fitted degree and the damage model (Eq. 7.14) had general applicability.

Table 7.5 The parameters of predicting points

\begin{tabular}{l|l|l|l}
\hline The predicting point item & Damage factor $(D)$ & TDF $(\mathrm{k})$ & The predicting time $(\mathrm{s})$ \\
\hline A & 0.325 & 0.022 & 14.50 \\
\hline B & 0.666 & 0.008 & 52.5 \\
\hline C & 0.980 & 0.023 & 67.5 \\
\hline D & 1.000 & 0.003 & 71.8 \\
\hline
\end{tabular}

Fig. 7.13 The tangent damage factor curve

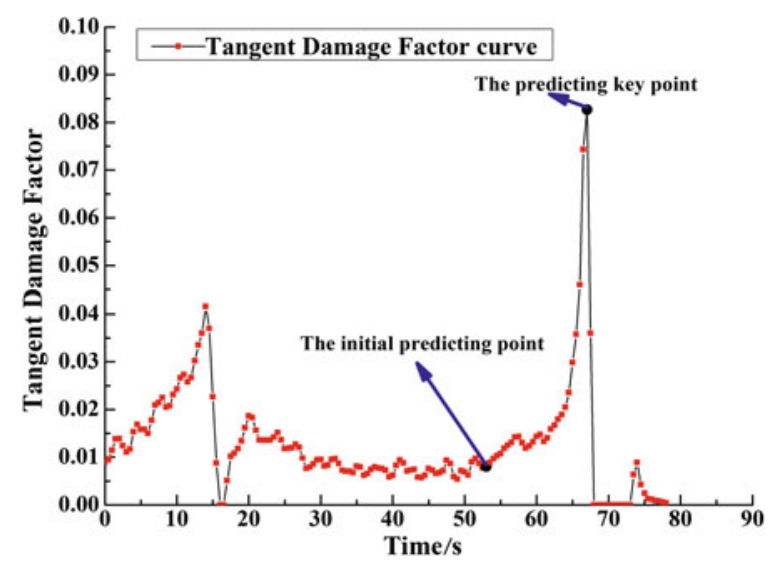




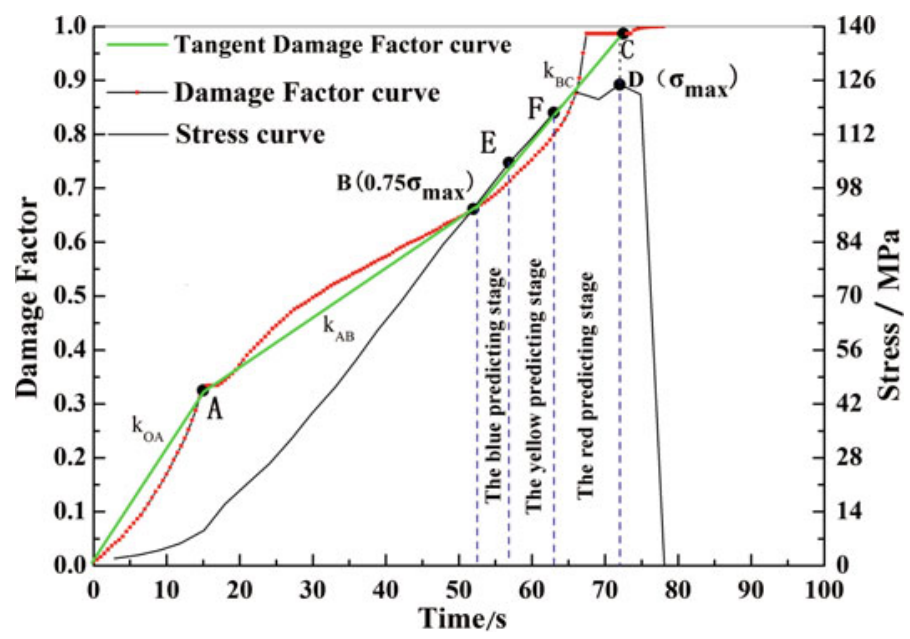

Fig. 7.14 The damage factor and the stress-time curve

The damage evolution characteristics were analysed using sample X5 as an example to analyze the process of rock failure. As shown in Fig. 7.14, DF and loaded-time curve of sample X5 were displayed. The evolution process of DF and loaded-time curves showed the characteristics of 'rapid growth-stable growthsharp growth-stability phased'. Therefore, the damage of pre-peak stress could be divided into four stages: the initial damage stage (OA), the continuous damage stage $(\mathrm{AB})$, the accelerated damage stage $(\mathrm{BC})$ and the stability damage stage.

(1) OA stage: DF curve corresponded to the original cracks compaction stage of stress-strain curve. The initial DF value of sample was 0 , and it rapidly increased because of obviously increased AE activities caused by the originally open micro-cracks. In this stage, DF curve had a concave shape. TDF increased gradually. As shown in Table 7.5, the slope of OA stage $\mathrm{k}_{\mathrm{OA}}$ value was 2.20, the slope of $\mathrm{AB}$ stage $\mathrm{k}_{\mathrm{AB}}$ value was 0.88 , and $\mathrm{k}_{\mathrm{OA}}>\mathrm{k}_{\mathrm{AB}}$. Therefore, point $\mathrm{A}$ was a turning point of DF value.

(2) $\mathrm{AB}$ stage: DF curve corresponded to the elastic deformation stage of stress-strain curve. In this stage, the straight line of DF value increased slowly because of less AE activity. TDF value had decreasing trend. However, it could be regarded as a constant. In this DF curve, point B was a sudden jump point of $\mathrm{DF}$, transitioning from the stable growth stage to the sharp growth stage. This characteristic, which was the sharp increase behaviour of TDF, was viewed as the predicting point of rock failure.

(3) BC stage: DF curve corresponded to the cracks growth of micro-damaging stage of stress-strain curve. In this stage, micro-cracks were generated constantly and cracks expanded rapidly. Meanwhile, AE activities increased significantly. 
DF value of BC stage increased more quickly than the OA stage. DF was a rising curve. TDF increases markedly. In Table 7.5, the slope of $\mathrm{BC}$ stage value $\mathrm{k}_{\mathrm{BC}}$ was 2.3 , and $\mathrm{k}_{\mathrm{BC}}>\mathrm{k}_{\mathrm{AB}}$.

(4) The latter stage: DF curve corresponded to the small part of failure stage of stress-strain curve. In this stage, the damage value remains nearly unchanged and TDF approximates zero.

\subsubsection{Predicting Models of TDF}

As shown in Fig. 7.14, in the compaction stage (OA), DF value of limestone sample increased markedly and $\mathrm{TDF} \mathrm{k}_{\mathrm{OA}}$ was relatively large. Then, in the liner elasticity stage $(\mathrm{AB})$, the DF value continued to increase, but there was a trend of slowing down. TDF $\mathrm{k}_{\mathrm{AB}}$ could be regarded as a constant. In this stage, the fracture of limestone sample did not occur. When DF value reached point B, and increased rapidly. The propagation and merged of cracks occurs. It would gradually develop until rock failure. Thus, point $\mathrm{B}$, the turning point from the stable growth stage to the sharp growth stage, could be regarded as the initial predicting point for the damage. When DF value exceeded point $\mathrm{C}$ to approaches 1 gradually, the limestone sample would begin to fail and become unstable. The peak point $\mathrm{D}$ in the curve was regarded as the predicting key point of rock failure. The identity of initial predicting point B was vital. Therefore, the initial predicting point could be identified based on the sudden change behaviour of TDF curve. As shown in Fig. 7.15, where a (14.5 s) was the first peak point of TDF curve; b (52.5 s) was the lowest point of TDF curve in the sudden rising process; $\mathrm{c}(66.1 \mathrm{~s})$ was the second peak point of

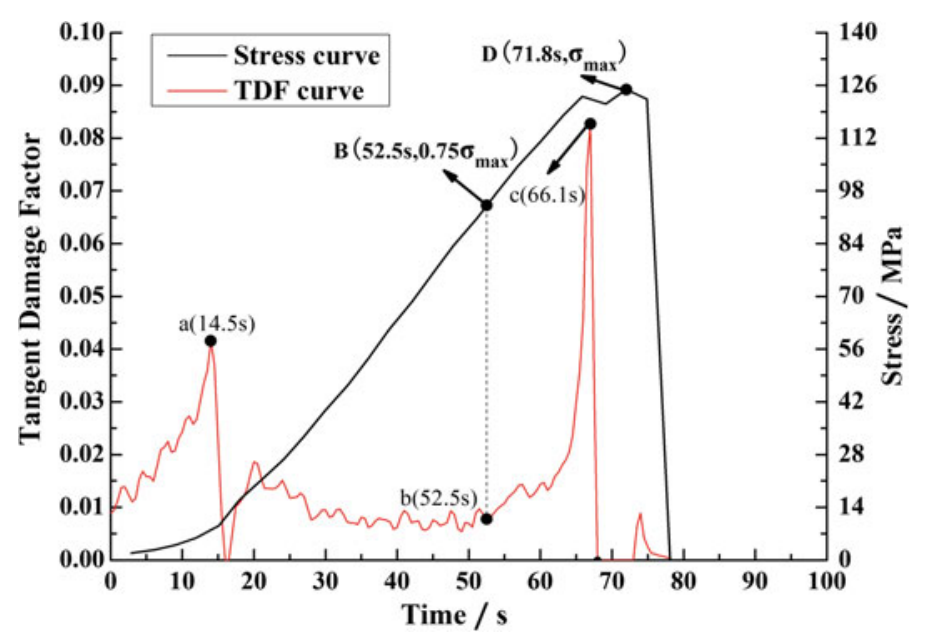

Fig. 7.15 Tangent damage factor and the stress-time curve 
TDF curve; $\mathrm{B}\left(52.5 \mathrm{~s}, 0.75 \sigma_{\max }\right)$ was the initial predicting point of stress curve corresponded the $\mathrm{b}$ point of TDF curve; D $\left(71.8 \mathrm{~s}, \sigma_{\max }\right)$ was the predicting key point of rock failure.

In the initial compaction stage, TDF increased markedly and eventually reached the peak point a $(14.5 \mathrm{~s})$. Then, it decreased sharply and finally reached the minimum point from 14.5 to $16 \mathrm{~s}$ in this period. That was also the transition phase of limestone for transiting from the original cracks compaction stage to the elastic deformation stage. After this stage, TDF tended to fluctuate steadily and the damage value increased more slowly. With the development of cracks, when TDF reached point b (52.5 s), TDF value increased sharply and was more markedly than that in the compaction stage. Finally, it suddenly jumped to peak point $\mathrm{c}(66.1 \mathrm{~s})$. Based on the loading time, point B of stress-strain curve could correspond to point $b$. The stress value of this point was $95.75 \mathrm{MPa}$, and it was approximately at $75 \%$ of peak strength. Point B was the sudden jump point for the DF value, transitioning from stable growth to sharp growth. It was the sudden jump point for transiting from the elastic deformation stage to the cracks growth of micro-cracking stage; the point $\mathrm{B}$ was defined as the initial predicting point. Beyond the TDF value point $\mathrm{c}$, TDF value decreased rapidly to 0 , eventually becoming smooth and steady. Based on the loading time, point $\mathrm{C}$ corresponded to the stress-strain curve. The stress value of this point was $125 \mathrm{MPa}$, and it was $98 \%$ the peak strength. The peak point $\mathrm{D}$ of stress-strain was defined as the predicting key point. Therefore, a damage-time-failure-predicting model was established based on the identification of TDF with the complete stress-strain curve. TDF, particularly, might play an important role in predicting rockburst.

To predict the failure of limestone more reasonably and accurately, time-divided predicting was proposed. Equal interval from the initial predicting point (B) to the predicting key point (D) was divided. TDF from $\mathrm{k}_{\mathrm{B}}$ to 0 was divided into three predicting stages: the blue predicting stage, the yellow predicting stage and the red predicting stage, which could yield the classification of predicting for limestone failure.

During the cracks growth of micro-damaging stage, when the stress was close to $90 \%$ peak strength, the micro-cracks, rock expansion and rock strain sped up. Meanwhile, the deformation of rock increased rapidly with the stress. Therefore, $0.917 \sigma_{\max }\left(\sigma_{\max }\right.$ is the compressive strength) was regarded as a predicting point. Based on the equal parting principle, stage BD was divided into three predicting stages, as shown in Fig. 7.14. The stage of B $\left(0.75 \sigma_{\max }\right)-\mathrm{E}\left(0.833 \sigma_{\max }\right)$ was the blue predicting stage; the stage of $\mathrm{E}\left(0.833 \sigma_{\max }\right)-\mathrm{F}\left(0.917 \sigma_{\max }\right)$ was the yellow predicting stage; the stage of $\mathrm{F}\left(0.917 \sigma_{\max }\right)-\mathrm{D}\left(\sigma_{\max }\right)$ was the red predicting stage.

As shown in Fig. 7.14, with the development of damage evolution, the damage value increased constantly. When the limestone was loaded to $52.5 \mathrm{~s}$ to reach the initial predicting point $\mathrm{B}\left(0.75 \sigma_{\max }\right)$, the limestone samples began to continuously generate cracks. The volume of limestone transited from the state of compression to the expansion and then reached the predicting point $\mathrm{E}$. The stage BE was defined as the blue predicting stage, which was the early stage of predicting, belonging to the transiting phase of elastic-plasticity. When exceeding the predicting point E, DF 
value had a small phase of sharply increasing until reached to the predicting point $\mathrm{F}$ $\left(0.917 \sigma_{\max }\right)$, which was defined as the yellow predicting stage. The stage EF was proposed as the middle predicting. When exceeding the predicting point $\mathrm{F}$, the speed of rock deformation increased quickly. Finally, the stress reached to the predicting key point $\mathrm{D}$. Therefore, the stress beyond the point $\mathrm{F}$ would enter the predicting key stage, and stage FD was defined as the red predicting stage.

\subsection{Experimental Investigation on Predicting Points Using Information Entropy Theory for Rock Failure}

\subsubsection{AE Dominant Frequency}

The Fast Fourier Transform (FFT) was proposed by Cooley and Tukey (1965). FFT is a type of fast computation method for the Discrete Fourier Transform (DFT). Based on the odd, even, imaginary, real and other characteristics of DFT, it is an improved form of DFT. FFT does not provide any findings different those of DFT. However, it substantially improves the arithmetic speed and efficiency of DFT, which is greatly useful for applying the DFT in a computer or digital system. In this chapter, FFT analysis was performed for all waveforms generated during rock failure, and the dominant frequencies were obtained. Sample X3 was used as an example to illustrate. The relationship between time-loading curves and dominant frequencies for the limestone sample was shown in Fig. 7.16.

The dominant frequencies of samples were obtained, and there were the following several characteristics:

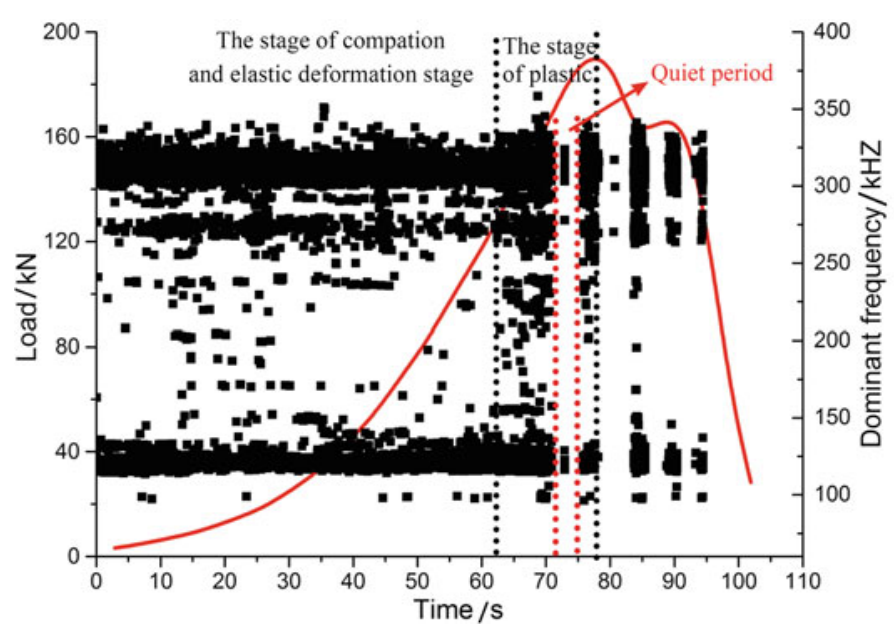

Fig. 7.16 Relationship between time-loading curves and dominant frequencies 
Table 7.6 Detail of dominant frequencies of sample X1

\begin{tabular}{l|c|c}
\hline Range $(\mathrm{kHz})$ & Amounts $(\mathrm{N})$ & Amount percentage $(\%)$ \\
\hline$[96.25,358.375]$ & 13,106 & 100 \\
\hline$[110,130]$ & 5231 & 40 \\
\hline$[275,340]$ & 6720 & 51 \\
\hline other & 1155 & 9 \\
\hline
\end{tabular}

(1) The sample had two frequency bands. The low-frequency band was at approximately $100-150 \mathrm{kHz}$, and the high-frequency band was at approximately $300-350 \mathrm{kHz}$.

(2) The high and low frequency bands of samples were generated simultaneously during the whole process of rock failure.

(3) With increasing load and deformation, the signals between two dominant frequency bands tended to increase.

Cai et al. (2007) noted out that the high-frequency band of AE corresponds to small-scale cracks, whereas the low-frequency band corresponds to large-scale cracks. Therefore, the change in the frequency bands indicated that rock deformation and failure involved a process of micro-crack initiation, propagation and linking. The dominant frequency distributions of rock sample had common characteristics.

As shown in Fig. 7.16, the dominant frequency distribution of the whole rockburst process was related to stress levels. In this chapter, the relationship between stress and frequency distribution characteristics was analysed based on sample X3. The range of dominant frequency values for the sample was 96,250 $358,375 \mathrm{~Hz}$, as shown in Table 7.6. In the rock compaction and elastic stages, the dominant frequencies were concentrated into two frequency bands, a low-frequency band of $110-130 \mathrm{kHz}$, and a high-frequency band of $275-325 \mathrm{kHz}$. In the plastic stage, the distribution of dominant frequencies in the two dominant frequency bands was more intensive, indicating that the cracks developed more rapidly. A large number of AE signals were released in this process. At the same time, numerous dominant frequency values appeared between two dominant frequency bands; the frequencies entered the broadband phase at this time. A complex AE rupture source was caused by the unstable propagation of cracks. The sample changed from comprising a single rupture source to comprising a variety of rupture sources. Meanwhile, it was observed that sample X3 had an obvious quiet period at $71-76 \mathrm{~s}$; stress was at $88.2 \%$ of the peak strength (Fig. 7.16). This phenomenon could be used as precursor information for rockburst.

\subsubsection{AE Dominant Frequency Entropy}

The dominant frequency entropy values were calculated based on Eq. (3.5). The relationships between load-time curves and the dominant frequency entropy values 


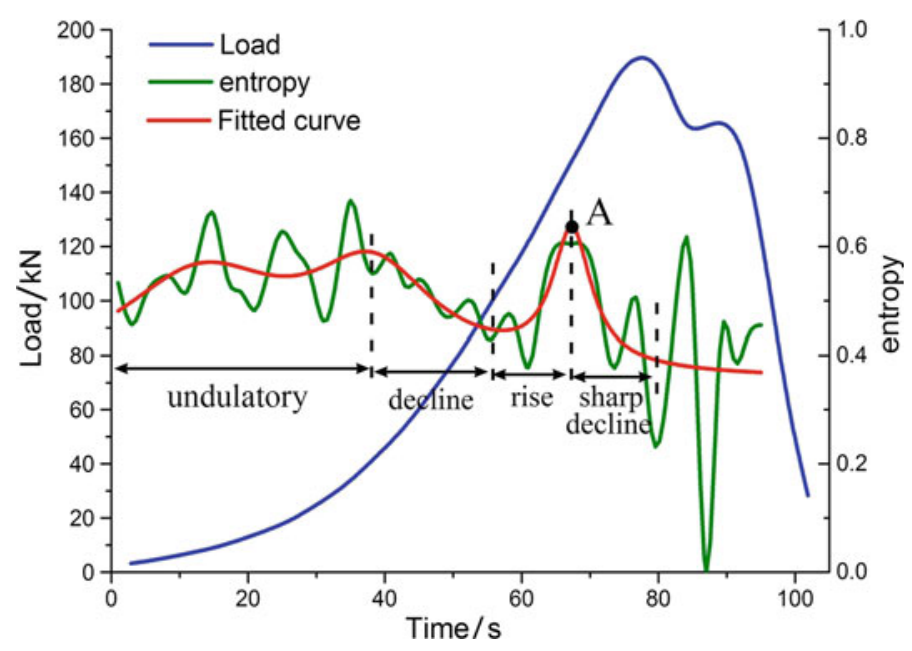

Fig. 7.17 Relationship between time-loading curve and entropy for sample X3

for the limestone samples were shown in Fig. 7.17. The dominant frequency entropy of sample exhibited the following characteristics: (1) The dominant frequency entropy values were between 0 and 1. (2) All of curves were in fluctuation. As shown in Fig. 7.17, the curve of sample X3 was fluctuating during the beginning of load, during the time interval between 0 and $38 \mathrm{~s}$. The disordered AE signals were mainly generated in the cracks compaction stage. With increased loading, the micro-cracks were generated in order. Therefore, the entropy curve was declined from 38 to $60 \mathrm{~s}$. Then, the curve appeared to rise slightly within the time interval between 60 and $69 \mathrm{~s}$. The continued generation of disordered AE signals was caused by micro-fracture formation. Then the micro-cracks initiated, propagated, and merged progressively into a macro-crack. Therefore, the entropy value decreased sharply in the time period from 69 to $78 \mathrm{~s}$. Therefore, the evolution model of sample X3 was 'wave-decline-small rise-sharp decrease'. The entropy evolution model of sample X3 illustrated that rockburst was a process of initiation and propagation of multiple cracks into a macro-crack. Meanwhile, these processes were synchronous.

\subsubsection{AE Dominant Frequency and Entropy}

Based on all collected AE waveforms, we calculated the dominant frequency values using the method of FFT spectrum analysis, and analyzed their distribution characteristics during rock failure. In the crack compaction and elastic deformation stages, these results showed that the distribution of dominant frequencies was concentrated into two frequency bands. Then, in the unstable crack propagation 
stage, the dominant frequencies were in the broadband range. Rock failure evolved from a single rupture source to a variety of rupture sources. For frequency evolution, several studies were studied. With the experiments in the laboratory and situ, Lu et al. (2012b) found that precursory AE (MS) signals was showed at tendency for the spectrum to move to the low-frequency band. Aftershock signals had spectra moving to the high-frequency band. Moreover, there was a negative relationship between predominant frequency of main shock signal and rockburst failure intensity. Bakker et al. (2016) performed AE experiment under triaxial compression in the laboratory, and found that low-frequency signals were generated during rock specimen failure because of movement of viscous fluid through cracks. Most prior studies were based on frequency distribution characteristics. Few studies focus on evolution models and the possibility of predicting rockburst using information entropy. In this chapter, based on the theory of information entropy, we further investigated the dominant frequency evolution during the overall process of rock deformation and failure. Meanwhile, information entropy evolution models were investigated. Notably, the entropy evolution followed the decreased pattern of wave rise-sharp. In order to better analyze information entropy evolution pattern, the curves of entropy evolution were fitted in Fig. 7.17. The phenomenon, when information entropy dropped sharply before rock failure, could be more intuitive to observe. Meanwhile, the entropy appeared at a maximum value, as shown at point A (Fig. 7.17). The maximum value point A appeared near the yield point. Then, the information entropy value decreased sharply. The change occurred synchronously with micro-crack merger and macro-crack formation. For the entropy value variation, in the author's opinion, the dissipative structure theory could explain this phenomenon. Prigogine (1969) pointed out that in an open system at a nonlinear region far from equilibrium, there would be continuous exchange of matter and energy with the external environment, and the system would undergo mutation when the external environment reached a certain condition, that was a nonequilibrium phase transition from disordered chaos into an ordered state of time, space or function. In the stage of compaction and elastic deformation stage, there was no macroscopic irreversible process inside the system, which was a balanced state. The information entropy presented wave variation. In the stable growth crack stage, the cracks stopped developing. After a certain time, the system might reach a macro state that did not change over time, was a nearly equilibrium state. In the unstable crack propagation stage, the cracks developed even though stress remained constant. As the micro-rupture expansion was a spontaneous dynamic process, the system was far from the equilibrium state. On one hand, the system absorbed energy from the outside; On the other hand, the system released energy because of cracks development. The macroscopic status of system changed with time, and the distribution of microcracks in the space was localized, that is, a phase transition from the disorder to ordered status. The entropy sharply decreased at this time. The dissipative structure was formation. With stress continuous increase, AE dominant frequencies changed from disordered chaos into an ordered status after stress reached point A. Therefore, the entropy value decreased sharply. Then, the loading 
of rock specimens entered the unstable cracks propagation stage. Micro-cracks propagated, merged progressively, and finally formed macro-cracks. The entropy value decreased sharply, which might provide precursory information for prediction rockburst. Therefore, we used point A as predicting key point of rockburst.

\subsection{Predicting Key Point Identification Using Traditional Monitoring Method}

In order to further study on predicting key point of rockmass deformation and failure, we analyzed the monitoring data of rockmass deformation from the stress-strain curve. However, how to analyze the development of plastic deformation by mechanical parameters is a question worthy of study. In this chapter, an instability predicting model and method was built using Tangent modulus. Meanwhile, this model was applied to Huize lead-Zinc Mine in China, which shows greater adaptability.

\subsubsection{Methods for Instrument Installation and Data Collection}

The detailed process to set up predicting methods for rockmass instability is as follows:

(1) Determine the monitoring and predicting area of rockmass, partitioning and numbering them. To get a distinct monitoring network, numbers correspond to the change of incremental stress and incremental displacement.

(2) Drill hole for stress and displacement monitoring. The bottom of drilling hole for stress monitoring should be located in the rockmass that was easy to produce instability and it cannot be located in the virgin of rockmass. The deepest anchor point of borehole for vibration twist-action multipoint displacement instrument should be located in the virgin of rockmass.

(3) Construct the borehole in the direction vertical to the excavation face according to the radial size of vibration twist-action multipoint displacement instrument. The drilling length should be more than 3 times the radius of excavation space. In general, the diameter of borehole is $45-76 \mathrm{~mm}$. Record the drilling length as $\mathrm{L}$ and the displacement value as $\Delta l$ (with units in $\mathrm{mm}$ ). The deepest displacement monitoring points should be located in the region of initial stress. The measurement range of multipoint displacement instrument is $0-20 \mathrm{~mm}$ with an accuracy of $0.2 \mathrm{~mm}$ and sensitivity of less than $0.01 \mathrm{~mm} / \mathrm{F}$. To master the strain change status in rockmass failure, the temperature measurement range is -40 to $140{ }^{\circ} \mathrm{C}$ with an accuracy of $\pm 0.50{ }^{\circ} \mathrm{C}$, the water pressure resistance is greater than 
$1 \mathrm{MPa}$, insulation resistance is greater than or equal to $50 \mathrm{M} \Omega$ and the anchor point numbers of about 3-5. Meanwhile, in order to master the deformation of rockmass, the deepest anchor point of multipoint displacement instrument should be located in the virgin of rockmass, the rest of anchor points evenly distributed in drilling hole.

(4) Install vibration twist-action multipoint displacement monitoring instruments, grouting, anchoring and installing the base of measuring head, displacement sensor and protection sleeve, lengthening the cable.

(5) Install the sensors. The direction of stress monitored by borehole stress monitoring instruments should correspond with the direction of displacement monitored by multipoint displacement monitoring instruments. After frequency stabilization (about $10 \mathrm{~min}$ ), the sensor frequency was record at this time as the initial frequency and input this data into the monitoring instruments as the zero point. Then, the monitoring instruments directly reported the incremental stress.

(6) Set the parameters of borehole stress monitoring instruments and vibration twist-action multipoint displacement monitoring instruments, connecting the borehole stress monitoring instruments and multipoint displacement monitoring instruments to the computer, analyzing the incremental stress and strain.

\subsubsection{Recognition Method of Predicting Hazards}

The author proposed that Tangent modulus is the slope of each point on the stress-strain curve. When rockmass is loaded, in the stage of elastic, Tangent modulus is equal to elastic modulus; in the inelastic stage, Tangent modulus reduces quickly. Tangent modulus is calculated as stress variation and strain variation ratio. Generally, rockmass is considered to be elastic-brittle material. Meanwhile, stress-strain curve for rockmass is nonlinear. Tangent modulus could be used to describe the macro modulus of inelastic stage. Therefore, based on the change of Tangent modulus in the yield area, the degree of rock deformation is determined and the predicting key point for rockmass failure is recognized.

In this chapter, the detail recognition process of predicting hazards is as follows:

(1) The incremental strain $\Delta \varepsilon$ value was got using the incremental displacement value $\Delta l$ and the deepest drilling anchor length $\mathrm{L}$. Based on the incremental stress value $\Delta \sigma$ and the incremental strain value $\Delta \varepsilon$, Tangent modulus $E_{t}$ was calculated by the following expression:

$$
E_{t}=\Delta \sigma / \Delta \varepsilon
$$

(2) According to the constitutive relation of force in rockmass, the change of Tangent modulus slope $k$ was used to predicting the rockmass failure. When Tangent modulus slope invariant or increases, which could infer that rockmass was in the compression or elastic phase and rockmass instability did not occur; 
When tangent modulus slope decreases, which indicated the rockmass was in the yield stage and the initiation, expansion, connection of secondary cracks occured, all of which leaded to rockmass failure. Thus, the inflection point, where Tangent modulus slope $k$ decreased, could be defined as the predicting key point for rockmass failure. That was the predicting recognition method for rockmass instability. The specific application of this method was presented in Chap. 9.

\section{7 b Value Characteristics with MS Activity in Deep Mining}

The dynamic hazard of mine refers to the rockburst, rock falling and other disasters, which is caused by rock stress field during mine operation. Stress concentration leads to rockmass failure because of initiation, development, merged, and rupture of micro-rupture, then micro-seismic (MS) event generates in the fracture process. In monitoring and predicting the dynamic hazard of mine, MS and AE monitoring are the basic methods. These methods are widely used in the excavation of deep mine.

Using MS monitoring system, the random variable parameter in one-group MS events can be obtained at a certain monitoring area in a period time, and the magnitude-frequency relation can be determined to analyze the characteristics of $b$ value. By MS monitoring, the status of micro-rupture distribution, the potential area of dynamic hazard in mine could be concluded and for which we can make a prediction in advance. Therefore, to identify the relation of $b$ value with rockmass failure and instability can help us to know the characteristic of precursory MS parameters produced by rockmass failure and provide a basis for mine safety production.

\subsubsection{The Magnitude-Frequency Relation-b Value}

Many experiments showed that MS event generated by mining excavation follows the Gutenberg-Richter criterion, as shown in Fig. 7.18. By analyzing the MS activity, the magnitude-frequency relation is suitable for all magnitude range. In the time interval $[a, b]$, there is an exponential relationship between the frequency and magnitude at a MS monitoring area as follows:

$$
n(M)=N_{0} \mathrm{e}^{-b M}
$$

In general, the logarithmic function form was put forward as follow: 
Fig. 7.18 Relationship between number of events and magnitude. From Gutenberg and Richter (1956)

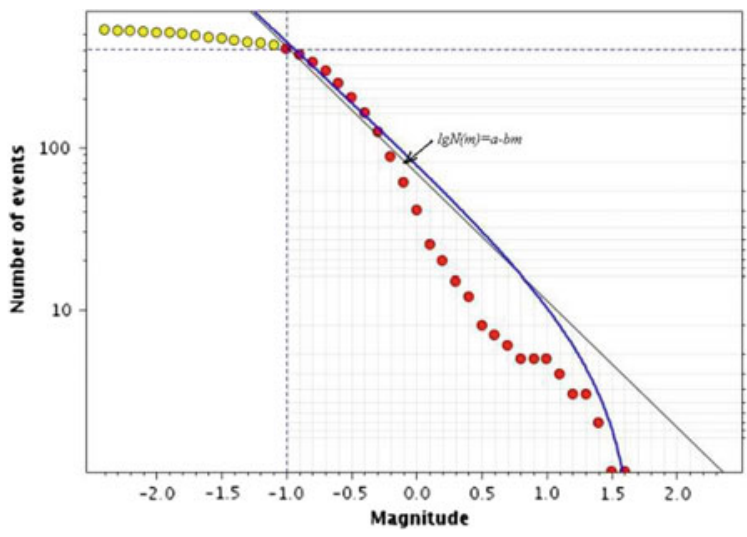

$\lg n(M)=a-b M$

where $a$ and $b$ are the unknown constants at a certain monitoring area; Constant a reflect the overall level of MS activity in this area and is viewed as MS activity parameter, which is correlated with the total MS events above the threshold magnitude; Constant $b$ describes the magnitude distribution of MS events, i.e., the relative number of small and large MS events in a certain time. Generally, $b$ value is close to 1 .

\subsubsection{Changes of b Value Caused by Rockmass Excavation}

$b$ value can reflect the proportional relationship of small and large earthquake events. The physical definition is that the change of $b$ values in one monitoring area, which means the degree of rock damage. The precondition is that $b$ values in this area have the unified monitoring capacity in a period. And the same earthquake magnitude above the lower limit magnitude could be monitored in the whole area. The change of $b$ values is correlated with the entire process of earthquake activity.

In the $8 \#$ orebody, the geology condition is very complicated with well developed faults and fragile rock-mass. Therefore, the dynamical hazard is easily occured during the stoping process. Because the stoping at the $1451 \mathrm{~m}$ level has overed, the 1331 and $1391 \mathrm{~m}$ levels are on upward stoping. The $1331-1451 \mathrm{~m}$ in the $8 \#$ orebody is the important area for stoping. The records of serious collapse caused in stope from August to December, 2007, were listed. Through the MS monitoring system, the parameters of MS events are obtained such as monitoring time, magnitude, $b$ value and the maximum magnitude, etc. The weekly $b$ values were recorded from August, 2007 to December, 2007 and listed. The specific application of this method is presented in Chap. 9. 
In conclusion, the value characteristics of $b$ can explain the dynamic hazard such as rockburst, stope roof fall, spalling. They provide an important role for predicting the rockmass instability, forecast accuracy and the improvement of safe work environment.

\subsection{Identification of Predicting Key Point Using AE/MS Monitoring System}

In laboratory experiment, the different variations of AE activity were obtained in different stress status. There were large difference between the performance characteristics of stress-strain variations and $\mathrm{AE}$ event activity rate. As shown in Fig. 7.19, in the compaction stage of initial damage, AE events were obviously increased and in an active state. However, the energy and magnitude of AE events were not sufficient to destroy the specimen. In the elastic stage, few AE events were produce, even tended to zero, which molecules and atoms were dislocated by force within the sample. When the stress reached the yield point, AE events increased dramatically, and then a relatively quiet period occurred. A large number of AE events would appear as a sharp increase in the peak strength until rockmass failure, unlike the quiet period. Therefore, a sharply decrease of AE events was used as the predicting point of rock failure, which did not indicate that rock instability was immediately obvious, but rock had entered the stage of cracks propagation. Rock damage status could be predicted with the further monitoring. In this chapter, the starting points of quiet period for AE events were determined as the predicting key points for the rock rupture and instability, which indicated the increase of rock

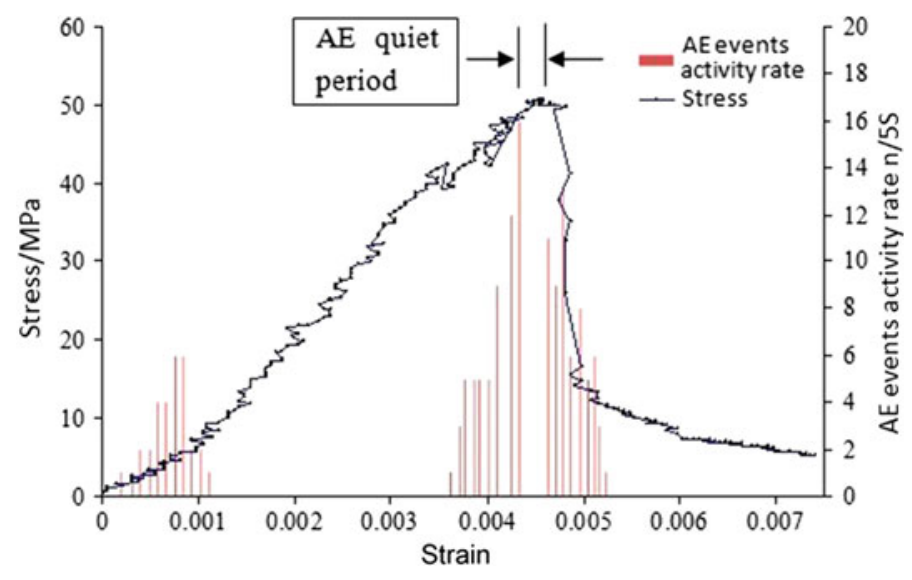

Fig. 7.19 The relationship between AE activity rate and stress-strain curve of rocky elastic-plastic materials. With kind permission from "PERGAMON-ELSEVIER SCIENCE LTD": Fig. 1, Wang (2014). All rights reserved 


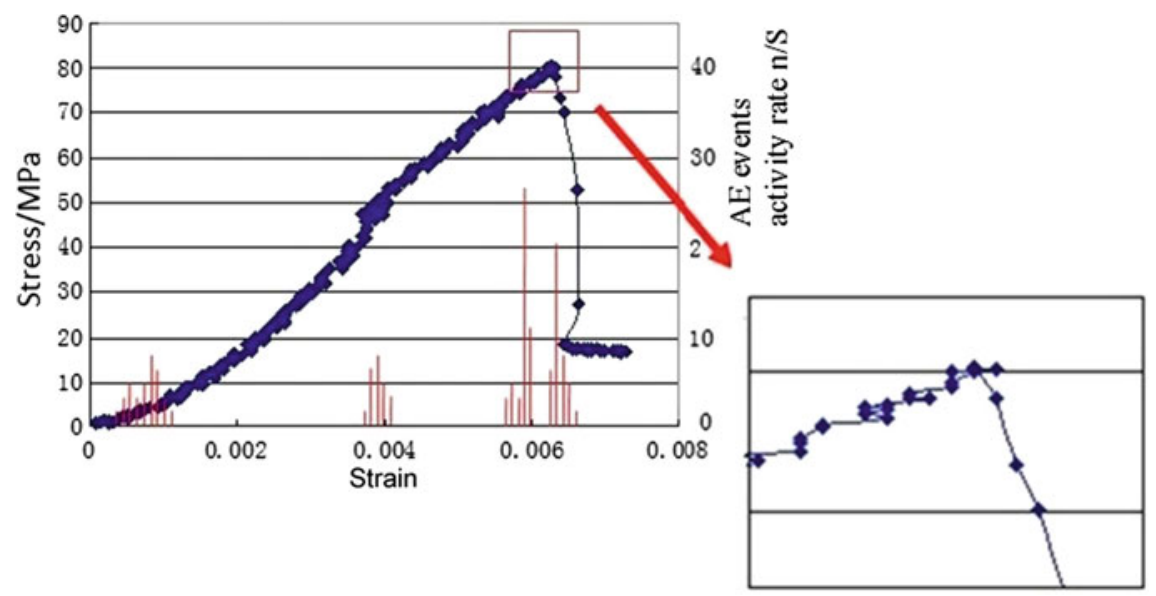

Fig. 7.20 The relationship between AE activity rate and stress-strain curve of rocky brittle materials. With kind permission from "PERGAMON-ELSEVIER SCIENCE LTD": Fig. 2, Wang (2014). All rights reserved

failure risk. With the increase of stress, the specimen would quickly enter a relatively stable period of AE events, which tended to be undermined. As rock sample is elastic-plastic material, the compaction and plastic stages were obvious in the deformation process. The time of AE quiet period is about $15 \mathrm{~s}$, which is relatively long. AE events appear the form of foreshocks-main shock-aftershocks. The predicting key points of rock failure could be easily identified by AE/MS monitoring system.

As rock is brittle material, it may be not easy to recognize the compaction and plastic deformation stages. Only a small deformation resulted in destruction and instability. Therefore, AE events, throughout the yield point, did not clearly and rapidly increase to enter the stage of rock instability and failure. Indeed, AE events exhibited mainshock-aftershock behavior. The predicting key points of this rock type were not easily identified by AE/MS monitoring, that is, the time of AE quiet period is about $2 \mathrm{~s}$, and it is relatively short and be easily lost, as shown in Fig. 7.20.

The main reason of $\mathrm{AE}$ relatively quiet period may be rock heterogeneity and particle distribution. When rock was loaded at the compaction stage, the nature cracks were compacted and a large number of small magnitude and low energy AE activity were appeared. Rock grains reached a status of balance. Then, rock reached the stage of elastic and a relative few stable AE activity. Under the constant load, new cracks were generated and propagated, which caused macro-cracks and a lot of great magnitude and high energy AE activity. Rock grains achieved another state of balance with the occurrence of AE quiet period before failure. When the balance of rock grains was broken again, rock achieved the peak strength and instability soon. The specific site application of this method is presented in Chap. 9. 


\subsection{Model of Multi-means and Synergistic Prediction for Rockburst}

In this section, we comprehensively consider several types of rockburst predicting methods mentioned in Sects. 7.2-7.8. Based on loading system, infrared radiation monitoring system and acoustic emission monitoring system, we designed the test system of rock failure to realize multi-means and synergistic prediction for rockburst. The test system is shown in Fig. 7.21.

As shown in Fig. 7.21, (1) Load/unload test system: load/unload the rock samples to obtain the stress-strain whole process curve and its corresponding data; (2) AE/MS monitoring system: synchronized with the load/unload process to monitor the process of rockburst, and obtain $\mathrm{AE}$ activity rate, energy, frequency and other AE parameters data; (3) Infrared radiation monitoring system: synchronized with the load/unload process to monitor the infrared thermogram change and record the infrared temperature data in whole rockburst process.

The model of multi-means and synergistic prediction for rockburst was shown in Fig. 7.22. All these methods, such as the infrared radiation temperatures, tangent modulus, load/unload response ratio and other ways; could effectively realize the prediction of rockburst. However, it was easy to misjudge, delay the information

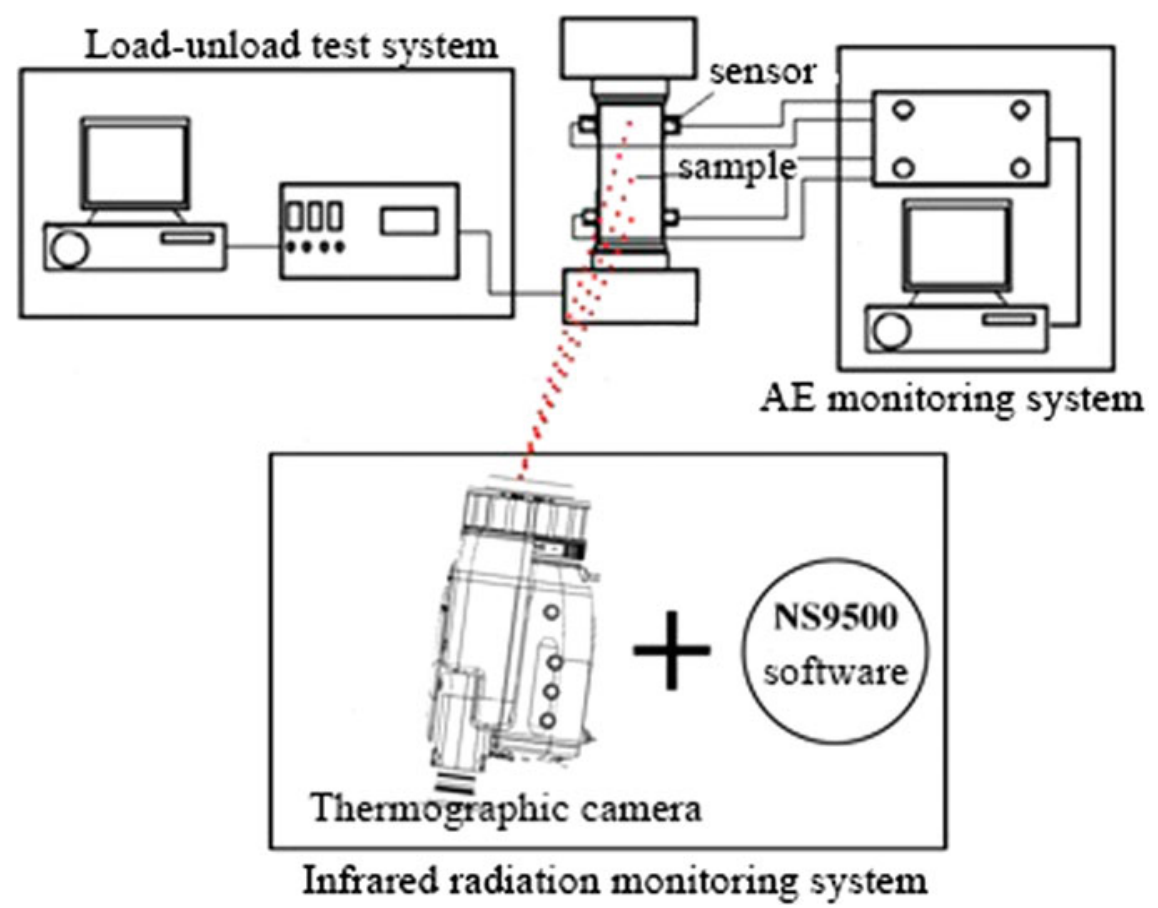

Fig. 7.21 Test system for coal failure synergetic prediction 


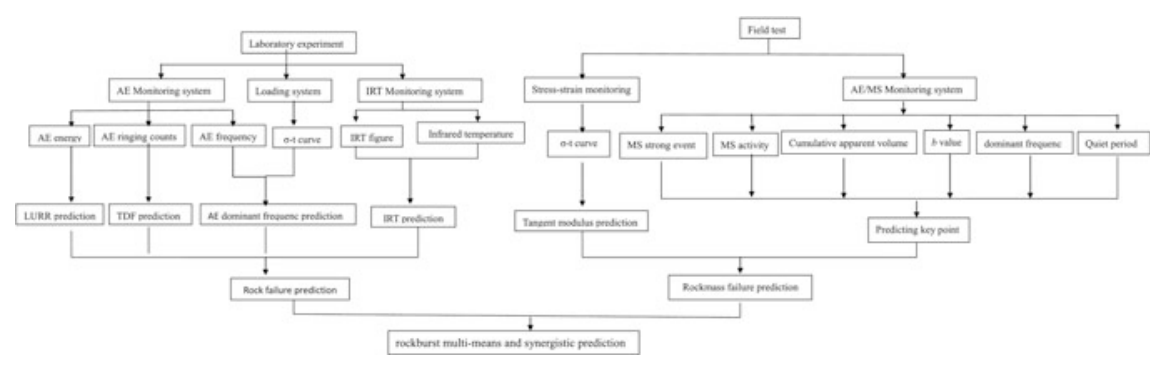

Fig. 7.22 Synergetic prediction for rockburst

and other issues using a single way to predict rockburst. The multi-means and coordinated synergistic prediction could make up for each other and verify each other, making it more reasonable, scientific and effective.

\subsection{Conclusion}

In this chapter, in view of single, hysteretic and poor effect prediction methods, we studied on the precursor information of rockbust using routine monitoring, infrared monitoring, AE/MS monitoring and other monitoring ways. The following conclusions were obtained:

(1) In the experiment, the mean temperature abnormality of "rising-dropping and rising" was observed in the later period of plastic deformation stage and could be suggested as the IRR precursor for rock failure. Meanwhile, the mean temperature increment interval of "dropping-rising" could be used as a predicting point for the coal failure.

(2) Based on the analysis of LURR evolution, LURR ratio $Y=1$ could be considered as the predicting point for rock failure.

(3) Based on TDF method, the sudden jump point, with respect to transiting from the state of stability to the state of rapid increase, was defined as the initial predicting point for the TDF. The peak point $\mathrm{C}$ was provided as the initial predicting point of red predicting stage for the TDF rapidly increasing peak point. The peak of stress-strain curve was defined as the predicting key point, the TDF value of which is 0 .

(4) The information entropy evolution models were a process of 'wave rise-sharp decrease'. The information entropy appeared at a maximum value before rockburst, and the maximum value was near the yield point. Then, the information entropy value decreased sharply, which could be viewed as a precursor of rockburst. Thus, the maximum value point could be used as a predicting key point for rockburst. 
(5) Based on the suddenly decrease of Tangent Modulus when the constitutive relation of rockmass came into the yield stage, the drop point of Tangent Modulus could be viewed as a precting key point for rock failure when rockmass passes through the elastic phase.

(6) MS activity parameters $b$ was sufficient and necessary conditions of rockmass failure. However, with the progress of mining and the increase of rockmass stress, it was important to grasp the patterns of MS activity parameters $b$ in the whole process of rockmass failure. This method could be used to predict dynamic disasters in deep mining.

(7) In the compaction stage, the low-magnitude and low-energy of MS events increases, and the high-magnitude and high-energy MS events sharply increased in entering the yield point. When MS events entered the relative quiet period, the predicting key point was identified as this position which indicated that the rockmass would increase the risk of rock instability.

(8) Based on predicting methods above mentioned, the multi-means and coordinated synergistic predicting method were designed, which made rockburst prediction more reasonable, scientific and effective.

\section{References}

Bakker RR, Fazio M, Benson PM, Hess KU, Dingwell DB (2016) The propagation and seismicity of dyke injection, new experimental evidence. Geophys Res Lett 43(5):1876-1883

Benson PM, Vinciguerra S, Meredith PG, Young RP (2010) Spatio-temporal evolution of volcano seismicity: a laboratory study. Earth Planet Sci Lett 297:315-323

Blake W, Hedley DGF (2003) Rockbursts: case studies from North American hard-rock mines. SME, Englewood, USA

Boltzmann L (1884) Ableitung des Stefan'schenGesetzes, betreffend die Abhängigkeit der Wärmestrahlung von der Temperaturaus der electromagnetischen Lichttheorie. Ann Phys 258:291-294

Brady BT (1969) The nonlinear mechanical behavior of brittle rock part I-stress-strain behavior during regions I and II. Int J Rock Mech Min Sci Geomech Abstr 6:211-225

Cai M, Kaiser PK, Morioka H et al (2007) FLAC/PFC coupled numerical simulation of AE in large-scale underground excavations. J Rock Mech Min Sci 44(4):550-564

Carpinteri A, Lacidogna G (2008) Acoustic emission and critical phenomena: from structural mechanics to geophysics. CRC

Carpinteri A, Lacidogna G, Niccolini G (2006) Critical behaviour in concrete structures and damage localization by acoustic emission. Key Eng Mater 312:305-310

Cook NGW (1992) Natural joints in rock: mechanical, hydraulic and seismic behaviour and properties under normal stress. Int J Rock Mech Min Sci Geomech Abstr 29:198-223

Cooley JW, Tukey JW (1965) An algorithm for the machine calculation of complex Fourier series. Math Comput 19(90):297-301

Cui CY, Deng MD, Geng NG (1993) Rock spectral radiation signatures under different pressures. Chin Sci Bull 38:1377-1382

Freund FT, Takeuchi A, Lau BWS et al (2007) Stimulated infrared emission from rocks: assessing a stress indicator. eEarth 2(1):7-16

Geng NG, Cui CY, Deng MD (1992) Remote sensing detection on rock fracturing experiment and the beginning of remote sensing rock mechanics. Acta Seismol Sin 14:645-652 
Genzano N, Aliano C, Filizzola C et al (2007) A robust satellite technique for monitoring seismically active areas: the case of Bhuj-Gujarat earthquake. Tectonophysics 431(1):197-210

Gutenberg B, Richter CF (1956) Magnitude and energy of earthquakes. Ann Geofis 9:1-15

He M, Miao J, Feng J (2010) Rock burst process of limestone and its acoustic emission characteristics under true-triaxial unloading conditions. Int J Rock Mech Min Sci 47:286-298

He MC, Zhao F, Zhang Y, Du S, Guan L (2015) Feature evolution of dominant frequency components in acoustic emissions of instantaneous strain-type granitic rockburst simulation tests. Rock Soil Mech 36(1):1-8 (in Chinese)

Kukshenko V, Elizarov S (2015) Experimental research on the load/unload response ratio (LURR) theory. Earthq Res China 3:31-39

Lu CP, Dou LM, Liu H, Liu HS et al (2012a) Case study on microseismic effect of coal and gas outburst process. Int J Rock Mech Min 53:101-110

Lu CP, Dou LM, Liu BA et al (2012b) Microseismic low-frequency precursor effect of bursting failure of coal and rock. J Appl Geophys 79:55-63

Lu CP, Dou LM, Zhang N et al (2013) Microseismic frequency-spectrum evolutionary rule of rockburst triggered by roof fall. Int J Rock Mech Min 64:6-16

Naiguang G, Ping YU, Mingde D et al (1998) The simulated experimental studies on cause of thermal infrared precursor of earthquakes. Earthquake 18(1):83-88

Planck M (1959) The theory of heat radiation. Dover, New York, USA

Prigogine I (1969) Structure, dissipation and life. Theoretical physics and biology. North-Holland Publishing Company, Amsterdam

Qiang ZJ, Xu XD, Dian CG (1990) Abnormal infrared thermal of satellite-forewarning of earthquakes. Chin Sci Bull 35:1324-1327

Rawat V, Saraf AK, Das J et al (2011) Anomalous land surface temperature and outgoing long-wave radiation observations prior to earthquakes in India and Romania. Nat Hazards 59 (1):33-46

Read MD, Ayling MR, Meredith PG, Murrell SA (1995) Microcracking during triaxial deformation of porous rocks monitored by changes in rock physical properties. Tectonophysics 245(3):223-235

Rudnicki JW, Sternlof KR (2005) Energy release model of compaction band propagation. Geophys Res Lett 32(16):1-4

Saraf AK, Rawat V, Banerjee P et al (2008) Satellite detection of earthquake thermal infrared precursors in Iran. Nat Hazards 47(1):119-135

Schiavi A, Niccolini G, Tarizzo P, Carpinteri A et al (2011a) Acoustic emissions at high and low frequencies during compression tests in brittle materials. Strain 47:105-110

Schiavi A, Niccolini G, Tarizzo P et al (2011b) Waveforms and frequency spectra of elastic emissions due to macrofractures in solids. In: Experimental and applied mechanics, vol 6. Springer, New York, pp 613-621

Schiavi A, Niccolini G, Carpinteri A, Lacidogna G (2013) Damage assessment in Syracuse limestone specimens by frequency analysis of elastic emissions. In: Experimental and applied mechanics, vol 4, pp 105-109

Senseny PE, Hansen FD, Russell JE et al (1992) Mechanical behaviour of rock salt: phenomenology and micromechanisms. Int J Rock Mech Min Sci Geomech Abstr 29:363-378

Shi X, Xu H, Wan Y et al (1994) The rock fracture under simulated tide force-laboratory study on the load/unload response ratio (LURR) theory. Acta Geophys Sin 37:631-636

Wang CL (2014) Identification of early warning key point for rockmass instability using acoustic emission/microseismic activity monitoring. Int J Rock Mech Min Sci 71(10):171-175

Wang CL, Wu AX, Liu XH et al (2009) Study on fractal characteristics of $b$ value with microseismic activity in deep mining. Proc Earth Planet Sci 1(1):592-597

Wasantha PLP, Ranjith PG, Shao SS (2014) Energy monitoring and analysis during deformation of bedded-sandstone: use of acoustic emission. Ultrasonics 54(1):217-226

Wu L, Cui C, Geng N, Wang J (2000) Remote sensing rock mechanics (RSRM) and associated experimental studies. Int J Rock Mech Min Sci 37:879-888 
Wu L, Wu H, Li G (2001) Experimental exploration to thermal infrared imaging for detecting the transient process of solid impact. Chin Sci Bull 46(10):872-877

Wu L, Liu S, Wu Y et al (2006a) Precursors for rock fracturing and failure-part I: IRR image abnormalities. Int J Rock Mech Min Sci 43:473-482

Wu L, Liu S, Wu Y et al (2006b) Precursors for rock fracturing and failure-Part II: IRR T-curve abnormalities. Int J Rock Mech Min Sci 43(3):483-493

Zhang L, Yu H, Yin X (2013) Failure potential evaluation in engineering experiments using load/ unload response ratio method. Pure Appl Geophys 170(1):237-245

Open Access This chapter is licensed under the terms of the Creative Commons Attribution-NonCommercial 4.0 International License (http://creativecommons.org/licenses/by-nc/ 4.0/), which permits any noncommercial use, sharing, adaptation, distribution and reproduction in any medium or format, as long as you give appropriate credit to the original author(s) and the source, provide a link to the Creative Commons license and indicate if changes were made.

The images or other third party material in this book are included in the book's Creative Commons license, unless indicated otherwise in a credit line to the material. If material is not included in the book's Creative Commons license and your intended use is not permitted by statutory regulation or exceeds the permitted use, you will need to obtain permission directly from the copyright holder.

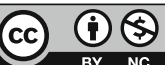




\section{Chapter 8 \\ Predicting Model of Rockburst Based on Nondeterministic Theory}

\subsection{Introduction}

Predicting is the basis of prevention and controlling of rockburst hazards. According to the predicting results, the feedback design of rock engineering and controlling measures are taken in time. It is of great theoretical and practical value for the safety and efficiency of deep mine. So far, there are many methods to judge rockburst tendency, such as rock integrity coefficient method, strength criterion discrimination method, rock brittleness index method, elastic energy index method, dynamic failure time method, rockburst energy ratio method, impact energy index method, impact tendency criterion method, and resistivity method. Most of these methods and judging indexes, which is only considered the individual factors, were one-sidedness, limitations and complications. Therefore, the influencing factors of rockburst are comprehensively based on the non-deterministic theory for established a more accurate rockburst predicting model. Rockburst is a complex dynamic instability phenomenon (Hedley 1992), which can occur during underground excavation in areas with large in situ stress. As a result of the sudden release of accumulated strain energy, rocks can be come loose, crack, and even eject violently (Canadian Rockburst Research Program 1996). As a result, rockbursts were considered a major technical challenge in deep mining. Duo to the characteristic of sudden, disruptive, and complex, the accurate prediction of rockbursts was difficult and an urgent problem need to be solved (Blake et al. 2003).

The phenomenon was discussed extensively by many scholars. Rockburst tendency is an important metric to quantify the risk and potential intensity of occurrences and grade the hazard of an affected mine. However, there are still no accurate prediction methods or effective control measures. In recent decades, many meaningful advances have been made by many scholars (Singh 1989; Dou et al. 2009; Marek 2009; Patynska et al. 2009; Marian 2011). Rockburst mechanism was better understand using some proposed some criteria, such as strength theory, stiffness theory and energy theory. These models could be explained the origin and mechanism of 
rockbursts, but were hard to apply in practice. In addition, several indexes were proposed to measure rockburst tendency, such as strength and brittleness, burst energy release, impact energy and rock integrity (Hoek et al. 1980; Cook 1965; Wiebols et al. 1968; Tan et al. 1991; Kidybiński 1981; Singh 1988; Hou et al. 1989). These criteria derived from the mechanical parameters obtained by testing rock samples. Some important values were compressive strength, tensile strength, capacity to store and release elastic strain energy, and surrounding rockmass stress and integrity.

In light of rockburst phenomenon complexity, the use of a single parameter was insufficient for predicting rockburst. Though AE, chip drilling, removal, vibration, and resistance methods were proposed and applied, each parameter was lacking in predictive power under isolation. As a multifactor, coupling induced dynamic hazard, it was essential to establish a calculation method to evaluate rockburst tendency involving the proper parameters. However, few studies were tried to combine the various factors relating to rockburst hazard. Recently, some interesting models were derived using artificial intelligence, such as a neural network (Chen et al. 2002), fuzzy theory (Adoko et al. 2013; Wang et al. 2015), and distance discriminant analysis method (Gong et al. 2007), along with other integrated analysis methods. These research results indicated that the occurrence of rockbursts was closely related to the mechanical properties of rockmass, the geological structure, and the surrounding stress. However, these attempts had not yet formed a complete theoretical system. Based on the Bayesian theory and Fuzzy element-matter theory, several critical factors were integrated into a single model for predicting rockburst tendency in this chapter.

Bayesian theory, which is successfully applied in many fields, provides a clear and a flexible method for making predictions using incomplete knowledge. Heckerman (Heckerman 1990) used a Bayesian framework to improve the process of medical diagnosis. Making full use of its strong information processing ability (Weidl et al. 2003), a Bayesian network was applied to the monitoring and management of industrial production processes. A Bayesian model was utilized for choosing investment ventures, and displayed a good ability to cope with future uncertainty (Kemmerer et al. 2002). In addition, Bayesian theory was used to identify faults in a computer system (Jensen et al. 2001). It was attempt that the tendency was predicted more precisely using these incomplete indexes of rockburst occurrence.

Professor Cai (1994) analyzed a large number of examples found that people in dealing with incompatibility issues must take things, features and the corresponding value to consider together. The main idea of this method was to make things to 'things, features, values' to describe and analyze. Matter-element analysis is an effective way to study matter-element and to solve incompatible problems in the real world. If the magnitude of matter is ambiguous, it constitutes a fuzzy incompatibility problem. Fuzzy matter-element analysis is the combination of fuzzy mathematics, and matter-element analysis can solve this kind of fuzzy things. In recent years, this highly practical theory and method were achieved many gratifying results in the field of engineering technology. In addition, artificial intelligence methods were used, 
such as fuzzy inference system (FIS) and adaptive neuro-fuzzy inference systems (Adoko 2013) and Rough set theory and genetic algorithms (Yu 2009). These were seismological theory and methods were used to predict the rockburst such as the peak velocity and dynamic energy, the seismic risk assessment method and mining and seismological parameters (Srinivasan et al. 1997; Li et al. 2011; Stewart 1995). The above research works indicated that the occurrence of rockburst was closely related to the strength of surrounding rockmass, geomechanics, geological structure, hydrogeology, and the construction sequence. Matter-element analysis theory was primarily used to study the problem of in compatibility (Wang et al. 2015). It could be also used for solving multiple-parameter evaluation problems by formalizing the problem and establishing the corresponding matter-element (Cai 1994; David et al. 1997; Chen et al. 2007; Liu et al. 2007). The improved fuzzy matter-element evaluation method was used to assess water quality, which achieved more reliable results than that using the traditional method (Liu et al. 2012). Based on the matter-element method (He et al. 2011) designed a model to evaluate the urban power net work planning, and Zhu (2010) analyzed coefficients of evaluation in rockburst. The empirical analysis showed that this model was reliable and feasible.

Therefore, in this chapter, Bayesian theory was demonstrated to be a reliable approach to address complex problems involving many variables with large uncertainties, and models that considered a multi-parameter space were better suited to predicting rockburst tendency than single-variable models. The main factors affecting of risk and intensity of rockbursts were used to make a Bayesian model. On the other hand, the main influencing factors of rockburst were considered based on the concept of matter-element analysis in combination with the fuzzy set and closeness degree rules. The entropy method was also integrated in the weight calculation in this model. An integrated rockburst multi-index evaluation model was established and used to predict the rockburst tendency in a case study.

\subsection{Predicting Model of Rockburst Based on Bayesian Theory}

\subsubsection{An Overview of Bayesian Theory}

A Bayesian model is a statistical analysis method commonly used to distinguish between types of samples. The primary procedure is based on an artificial familiarity with known samples and possible attendant consequences. Firstly, the empirical probability and covariance of each classification was analysed and calculated. Then, a discriminant function was formulated to grade samples. Finally, a posterior probability was calculated to verify the original evaluation. New samples could be easily classified after being input into model (Ren and Yu 1999; Gao 1999). 


\subsubsection{Empirical Probability}

A sample set can be divided into $k$ categories $\mathrm{G}_{1}, \mathrm{G}_{2}, \ldots \mathrm{G}_{k}$ according to a certain criterion. Assuming each sample has $m$ factors, $\mathrm{x}_{1}, \mathrm{x}_{2}, \ldots \mathrm{x}_{m}$, that are normally distributed, a given sample can be expressed as a $m$-dimensional array $X_{\mathrm{j}}^{(\mathrm{i})}=\left[\left(\mathrm{x}_{1}, \mathrm{x}_{2}, \ldots \mathrm{x}_{m}\right)_{j}^{(i)}\right]^{T}$, where $\mathrm{i}=1,2,3, \ldots k, \mathrm{j}=1,2,3, \ldots \mathrm{n}_{i}$, and $X_{\mathrm{j}}^{(\mathrm{i})} \in G_{i}$.

If there are a sufficient number of samples, the "empirical probability", which is the probability that a single sample will be classified into $G_{i}$, it can be expressed as

$$
p_{i}=\frac{n_{i}}{n_{1}+n_{2}+, \ldots, n_{k}}=\frac{n_{i}}{\sum_{i=1}^{k} n_{i}}
$$

\subsubsection{Mean Values and Covariance}

Moment estimation of mean values and variances can be introduced to generalize the distribution characteristics of $i$-th category:

$$
\begin{gathered}
\mu X^{(\mathrm{i})}=\bar{X}^{(\mathrm{i})}=\frac{1}{n_{i}} \sum_{j=1}^{n_{i}} X_{j}^{(i)} \\
S_{\mathrm{i}}^{2}=\frac{1}{n_{i}-1} \sum_{j=1}^{n_{i}}\left(X_{j}^{(i)}-\bar{X}^{(i)}\right)\left(X_{j}^{(i)}-\bar{X}^{(i)}\right)^{T} \\
\sum=\frac{1}{\sum_{i=1}^{\mathrm{k}}\left(n_{i}-1\right)} \sum_{i=1}^{k}\left(n_{i}-1\right) S_{i}^{2}
\end{gathered}
$$

where $\bar{X}^{(\mathrm{i})}, \mu X^{(\mathrm{i})}, S_{\mathrm{i}}^{2}$ refer to the mean values, expectation of mean values, and covariance of $i$ th category, respectively. Additionally, $\Sigma$ stands for the covariance matrix of overall sample population.

\subsubsection{Empirical Discriminant}

In this Bayesian model, the empirical discriminant of sample classification can be expressed as:

$$
\omega_{\mathrm{i}}(\mathrm{X})=\mu_{\mathrm{i}}^{T} \Sigma^{-1} X-\frac{1}{2} \mu_{\mathrm{i}}^{T} \Sigma^{-1} \mu_{\mathrm{i}}+\ln p_{i}
$$

The discriminating rule can be simplified to be:

If $\omega_{\mathrm{i}}(\mathrm{X})=\max _{1 \leq j \leq k} \omega_{\mathrm{j}}(\mathrm{X})$, then $X \in G_{i}$. 


\subsubsection{Posterior Probability and Verification}

A Bayesian distance discriminant method was used to separate the samples. The distance of a given sample $X=\left(\mathrm{x}_{1}, \mathrm{x}_{2}, \ldots \mathrm{x}_{m}\right)^{T}$ to the $i$-th category's centroid can be calculated as:

$$
d_{j}^{2}(X)=\left(X-\mu_{j}\right)^{T} \Sigma^{-1}\left(X-\mu_{j}\right)-2 \ln p_{j}
$$

so the posterior probability that $X$ belongs to the $i$-th category can be calculated as follow:

$$
P\left(G_{\mathrm{j}} \mid X\right)=\frac{\exp \left[-\frac{1}{2} d_{j}^{2}(X)\right]}{\sum_{i=1}^{k} \exp \left[-\frac{1}{2} d_{i}^{2}(X)\right]}
$$

If $P\left(G_{\mathrm{j}} \mid X\right)>50 \%$, then $X \in G_{i}$.

This confirms the previous result.

\subsubsection{Key Factors of Rockburst Tendency}

\subsubsection{Induced Factors of Rockburst}

Numerous engineering datasets showed that rockbursts were usually occurred in hard-rock zones that were mostly intact and exhibited high strength, while there were few rockbursts in soft-rock areas. Thus, the occurrence of rockbursts was strongly related to the mechanical properties of surrounding rockmass. The development and triggering of rockburst was a physical process of gradual energy storage and sudden instability, which showed as a large energy release. Laboratory tests showed that these rocks were brittle but high-strength with linear elastic characteristics. The tests also showed that these rocks had the characteristics of elastic-brittle failure and that the elastic modulus was relative high. As a result, they were prone to brittle failure in the high-stress regime. Excavation could result in stress redistribution and concentration of surrounding rockmass. Once local brittle failure occurs, the accumulated energy was quickly released, caused easily a rockburst.

There was a close relationship between the occurrence of a rockburst and the characteristics of in situ stress concentration. Under the same geological conditions, high stress was concentrated in the local rock. Some rock zones had instead low crustal stress levels. An intact rock with high crustal stress usually had a high elastic modulus, which meaned a large capacity to store strain energy. High stress locations were especially prone to occur rockbursts, especially zones not in a hydrostatic status. In the stress concentration zones, most rockbursts were attributable to the discordance of three dimensional stresses, which leaded to the shear failure of rock, rapid energy release and rockburst. 
Table 8.1 Impact factors of rockburst tendency

\begin{tabular}{l|l|l|l|l|l|l}
\hline Scholars & \multicolumn{7}{|l}{ Impact factors } \\
\hline & $\sigma_{1}$ & $\sigma_{\theta}$ & $\sigma_{1}$ & $\sigma_{\mathrm{c}}$ & $\sigma_{\mathrm{t}}$ & $W_{\text {et }}$ \\
\hline Russenes (1974) & & $\sqrt{ }$ & & $\sqrt{ }$ & & \\
\hline Turchaninov (1981) & & $\sqrt{ }$ & $\sqrt{ }$ & $\sqrt{ }$ & & \\
\hline $\begin{array}{l}\text { Hoek and Brown } \\
(1980)\end{array}$ & & $\sqrt{ }$ & & $\sqrt{ }$ & & \\
\hline Barton (1974) & $\sqrt{ }$ & & & $\sqrt{ }$ & $\sqrt{ }$ & \\
\hline Kidybinski (1981) & & & & & & $\sqrt{ }$ \\
\hline Tao (1987) & $\sqrt{ }$ & & & $\sqrt{ }$ & & \\
\hline Xu (2002) & & $\sqrt{ }$ & & $\sqrt{ }$ & & \\
\hline Wang (1998) & & $\sqrt{ }$ & & $\sqrt{ }$ & & \\
\hline Zhang (1991) & & $\sqrt{ }$ & & $\sqrt{ }$ & & $\sqrt{ }$ \\
\hline
\end{tabular}

Deformations within rockmass were dominated by elastic strain, and most of brittle rock with high strength belonged to this category. Conversely, rockmass dominated by plastic deformation could store less energy. In general, high-strength, brittle rock at underground engineering sites was most likely to induce rockburst.

With respect to energy, rock deformation and failure was the result of energy dissipation and release. The relationship between the dissipated energy and released energy was analysed along the uniaxial loading path; therefore, it might be an effective method that rockburst tendency characterized using the energy index. The deformation characteristics prior to rock failure could be approximately captured by the stress-strain behaviour (Müller 2007).

Rockburst tendency was used to characterize the risk and intensity of these events. The severity could be estimated using different quantitative or qualitative methods. Generally, rockburst intensity was divided into four levels, namely, strong rockburst, moderate rockburst, weak rockburst, and none.

Most scholars studied rockburst tendency using the mechanical properties of field rocks, while some others believed that the stress status of surrounding rockmass was more critical. In the previous literature, one or more factors were used to formulate variables to evaluate rockburst tendency (Wang et al. 2015). Some factors adopted here were listed in Table 8.1. Rockburst tendency was associated most often with $\sigma_{c}$ and $\sigma_{\theta}$, and followed by $\sigma_{1}$ and $W_{\text {et }}$.

There was an extremely complex nonlinear relationship between rockburst and its influencing factors. The selection of rockburst tendency evaluation factor should reflect the basic characteristics of rock and combine the various indexes.

\subsubsection{Critical Factors}

There was a complicated, nonlinear relationship between rockburst and its induced factors. In order to make effective prediction of rockburst, multiple variables were considerated by the integrated indexes. The uniaxial compression strength $\sigma_{\mathrm{c}}$ and 


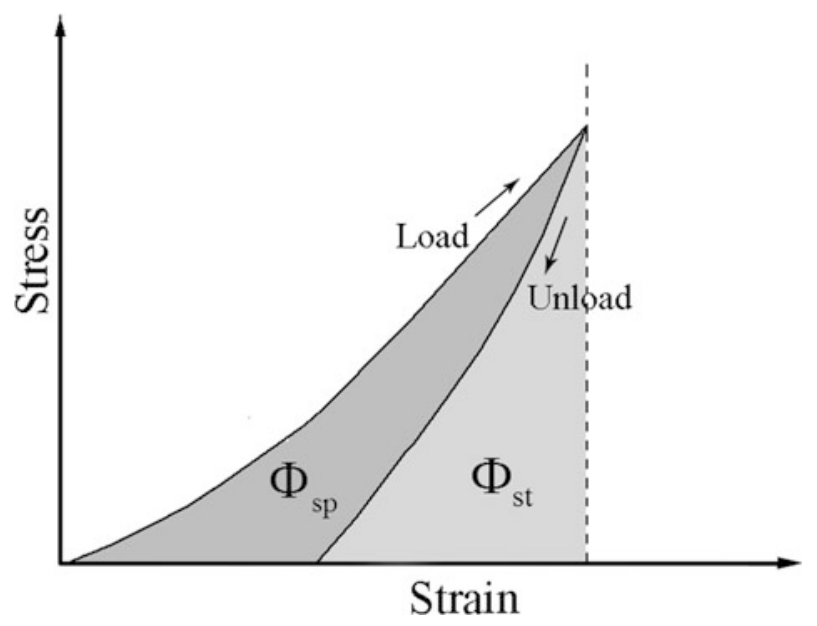

Fig. 8.1 Energy index of rockburst testing. From Kidybiński (1981)

uniaxial tensional strength $\sigma_{\mathrm{t}}$ were the main factors influencing the mechanical properties of rocks. The rock strength-stress ratio $R_{b}$, that is, the brittleness coefficient, could be applied to predict rockburst tendency. Generally, larger $R_{b}$ values were associated with greater rockburst risks. Where $W_{\text {et }}$ was determined by the uniaxial load/unload curve. Obtaining the load/unload path, rock was usually loaded to $70-80 \%$ peak stress, and then unloaded to zero. $W_{\text {et }}$ equaled to the ratio of released energy to the dissipated energy along the load/unload path. As shown in Fig. 8.1, $\Phi_{\mathrm{sp}}$ was the area between the loading path, and $\Phi_{\mathrm{st}}$ was the area under unloading path, and the energy index $W_{\text {et }}$ was calculated as the area ratio $\Phi_{\mathrm{sp}} / \Phi_{\text {st }}$, as shown in Fig. 8.1.

This elastic energy release index of rocks was used to evaluate rockburst tendency. Moreover, the axial stress status of surrounding rockmass was taken into account using Russense's criterion $R_{t}$, which also greatly influenced the risk of rockburst. Multi-paramters, such as physical and mechanical properties, the elastic energy release index, and the stress in the initial rockmass, were considered from different perspectives in these methods. A number of single-variable methods were widely recognized and applied, and three of them are listed in Table 8.2.

Table 8.2 Three classic discriminants for rockburst tendency

\begin{tabular}{l|l|l|l|l|l}
\hline \multirow{2}{*}{ Methods } & Formulas & \multicolumn{4}{l}{ Rockurst grades criterion } \\
\cline { 3 - 6 } & & No & Weak & Medium & Strong \\
\hline $\begin{array}{l}\text { Rock brittleness } \\
\text { index }\end{array}$ & $R_{b}=\frac{\sigma_{c}}{\sigma_{t}}$ & $>40.0$ & $\begin{array}{l}26.7- \\
40.0\end{array}$ & $\begin{array}{l}14.5- \\
26.7\end{array}$ & $<14.5$ \\
\hline Russenses's method & $R_{\theta}=\frac{\sigma_{\theta}}{\sigma_{c}} \sigma_{\theta}=\sigma_{1}-\sigma_{3}$ & $<0.2$ & $0.2-0.3$ & $0.3-0.55$ & $>0.55$ \\
\hline Kidybinski's method & $W_{\mathrm{et}}=\frac{\Phi_{\mathrm{sp}}}{\Phi_{\mathrm{st}}}$ & $<2.0$ & $2.0-3.5$ & $3.5-5.0$ & $>5.0$ \\
\hline
\end{tabular}


The grading criteria for the different methods were presented on Table 8.2. Each row represented a different method, and the columns gave the criteria within each for grading rockbusts into the four intensity levels. Three indexes $R_{b}, R_{\theta}$ and $W_{\text {et }}$ were used as the critical factors in this book.

\subsection{Predicting Model of Rockburst Based on Fuzzy Matter-Element Theory}

\subsubsection{Fuzzy Matter-Element and Composite Fuzzy Matter-Element}

For a given object with $M$ items to be evaluated and the characteristic $C$ is $x$, the sequential matter-element $\mathrm{R}=(M, C, x)$ can be constituted. Assumed that $x$ is fuzzy, $M$ has $n$ characteristics $C_{1}, C_{2}, \ldots, C_{n}$ and the corresponding $n$ values are $x_{1}, x_{2}, \ldots, x_{n}, R$ is called $n$-dimensional fuzzy matter-elements. If $n$ dimensional matter elements are combined with $m$ items together, it is a $n$-dimensional composite fuzzy matter element $R_{n m}$, which can be expressed as:

$$
R_{n m}=\left[\begin{array}{lllll} 
& M_{1} & M_{2} & \cdots & M_{m} \\
C_{1} & \mu\left(x_{11}\right) & \mu\left(x_{12}\right) & \cdots & \mu\left(x_{1 m}\right) \\
C_{2} & \mu\left(x_{21}\right) & \mu\left(x_{22}\right) & \cdots & \mu\left(x_{2 m}\right) \\
\vdots & \vdots & \vdots & \ldots & \vdots \\
C_{n} & \mu\left(x_{n 1}\right) & \mu\left(x_{n 1}\right) & \cdots & \mu\left(x_{n m}\right)
\end{array}\right]
$$

where $\mathrm{R}_{n m}$ is $n$-dimensional matter element for $m$ items, $M_{i}$ is the $i$ th item $(i=1,2$, $\ldots, \mathrm{m}), \mathrm{C}_{j}$ is the $j$ th characteristic $(j=1,2, \ldots, \mathrm{n}), \mu\left(x_{i j}\right)$ is the fuzzy value of $\mathrm{x}_{i j}$, which is the corresponding value for $\mathrm{M}_{i}^{\prime} \mathrm{s} j$-th characteristic $\mathrm{C}_{j}$. In this study, is $\mu\left(x_{i j}\right)$ is standardized using the principle of preferable fuzzy membership grade, which refers to the following methods:

The larger the more optimal model: $\mu_{i j}=X_{i j} / \max X_{i j}$

The smaller the more optimal model:

$$
\mu_{i j}=\frac{\min X_{i j}}{X_{i j}}
$$

where $X_{i j}$ the value of corresponding characteristics $j$ of $i$-th item; $\max X_{i j}, \min X_{i j}$ refer to the maximum and minimum value of all $X_{i j}$, respectively. 


\subsubsection{Standard Fuzzy Matter-Element and Difference Square Composite Fuzzy Matter-Element}

Standard fuzzy matter-element $\mathrm{R}_{0 n}$ is the maximum or minimum value of preferable membership grade for each evaluation method. In this chapter, the maximum value is the optimal value, which also means the preferable membership grade for each index is $1 . \Delta_{i j}(\mathrm{i}=1,2, \ldots, \mathrm{n}, \mathrm{j}=1,2, \ldots, \mathrm{m})$ is the square sum of element difference between standard fuzzy matter-element $R_{0 n}$ and composite fuzzy matter-element $R_{n m}$. The difference square composite fuzzy matter-element is $\Delta_{i j}$, namely $\Delta_{i j}=\left(\mu_{0 j}-\mu_{i j}\right)^{2}$, which can be expressed as:

$$
\Delta_{i j}=\left[\begin{array}{lllll} 
& M_{1} & M_{2} & \cdots & M_{m} \\
C_{1} & \Delta_{11} & \Delta_{12} & \cdots & \Delta_{1 m} \\
C_{2} & \Delta_{21} & \Delta_{22} & \cdots & \Delta_{2 m} \\
\vdots & \vdots & \vdots & \cdots & \vdots \\
C_{n} & \Delta_{n 1} & \Delta_{n 2} & \cdots & \Delta_{n m}
\end{array}\right]
$$

\subsubsection{Weight Coefficients Determined by Entropy Method}

Entropy theory is an objective weighting method that entropy value reflects the chaos level of data. The smaller the entropy value, the smaller the chaos level of data. Therefore, information entropy can be used to evaluate the order degree and utility of obtained information, namely it is to determine the index weight by a judgment matrix constituted by an array of evaluation indexes. It makes the assessment more realistic because it can eliminate human disturbance in the calculation of each index weight. The calculation steps are described as follows (Cai 1994):

(1) Constructing a judgment matrix with $n$ evaluation indexes for $m$ matters

$$
\mathrm{R}=\left(x_{i j}\right)_{n m} \quad(i=1,2, \ldots, n ; j=1,2, \ldots, m)
$$

(2) Normalizing judgment matrix to get the normalized judgment matrix B

$$
b_{i j}=\frac{x_{i j}-x_{\min }}{x_{\max }-x_{\min }}
$$


where $x_{\max }$ and $x_{\min }$ are the most satisfied and the most unsatisfied value for different matters under same method, respectively. For example, the smaller the value the more satisfied or the larger the value the more unsatisfied.

(3) According to the definition of entropy for $n$ evaluation indexes of matters, the entropy of evaluation indexes is defined as

$$
\begin{gathered}
H_{i}=\frac{-1}{\ln m} \sum_{j=1}^{m}\left(f_{i j} \ln f_{i j}\right) \\
f_{i j}=\frac{1+b_{i j}}{\sum_{j=1}^{m}\left(1+b_{i j}\right)}(i=1,2, \ldots, n ; j=1,2, \ldots, m)
\end{gathered}
$$

(4) Calculating entropy weight $w$

$$
w_{i}=\frac{1-H_{i}}{n-\sum_{i=1}^{n} H_{i}}
$$

where $\sum_{i=1}^{n} w_{i}=1$

\subsubsection{Closeness Degree and Comprehensive Evaluation}

Closeness degree is the proximity degree between the evaluated samples and a standard sample. Therefore, each evaluation scheme can be sequenced based on the closeness degree, or be classified according to the closeness degree of standard value. Considering the detailed evaluation significance, the Euclid closeness degree $\rho H_{j}$ is used as the evaluation standard, using $(\cdot,+)$ algorithm (first by multiplication, then by addition) to calculate and build up closeness degree for the composite fuzzy matter-element $R_{\rho H}$ (Cai 1994):

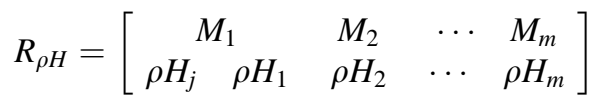

where $\rho H_{j}=1-\left(\sum_{i=1}^{n} K_{i} \Delta_{i j}\right)^{1 / 2}(\mathrm{j}=1,2, \ldots, \mathrm{m})$, and $K_{i}$ is the relational degree. 


\subsection{Conclusions}

By considering multiple factors, the new model can overcome the limitations of single-factor methods. In this chapter, most of parameters and methods were considered in order to eliminate subjective judgments. These variables included rock brittleness index $R_{b}$, Russense's $R_{\theta}$, Kidybinski's $W_{\text {et }}$, the surrounding rockmass stress $\sigma_{1}$ and $\sigma_{\theta}$, and rock strength $\sigma_{c}$ and $\sigma_{t}$.

The occurrence of rockburst was correlated not only with the physi-mechanical parameters of rockmass, but also with the surrounding mining environment. This research was intended to advance the development of predicting rockburst model based on fuzzy matter element method and measurement data.

Proposed model was based on fuzzy matter-element analysis, combining with the concept to Euclid closeness degree, an integrated rockburst multi-index predicted model was proposed. In this model, the rock brittleness $(B)$, the major principal stress $\left(\sigma_{1}\right)$, rock integrity $\left(k_{V}\right)$ and impact energy parameters $\left(W_{C F}\right)$ were all considered. At the same time, the entropy theory was introduced to determine the weight of each evaluation index, preventing the subjectivity of weight distribution. Finally, we recommend the fuzzy matter-element model on predicting rockburst method in field.

\section{References}

Adoko AC, Gokceoglu C, Wu L (2013) Knowledge-based and data-driven fuzzy modeling for rockburst prediction. Int J Rock Mech Min Sci 61:86-95

Barton N, Lien R, Lunde J(1974) Engineering classification of rock masses for the design of tunnel support. Rock Mech 6(4):189-236

Blake W, Hedley DGF (2003) Rockbursts: case studies from North American hard-rock mines. SME

Cai W (1994) Matter element model and its application. Scientific and Technical Documents Publishing House, Beijing, pp 256-68 (in Chinese)

Canadian Rockburst Research Program (1996) Rockburst research handbook: a comprehensive summary of five years of collaborative research on rockbursting in hard rock mines. CAMIRO Mining Division, CRRP, Sudbury ON

Chen HJ, Li NH, Nie DX, Shang YQ (2002) Artificial neural network model for prediction of rockburst. Chin J Geotech Eng 24(2):229-232 (in Chinese)

Chen HQ, Wang N, Sheng YR (2007) Application of fuzzy matter element model in evaluation of water security based on Euclid approach degree. J Xi'an Univ Technol 23:37-42 (in Chinese)

Cook NGW (1965) The failure of rock. Int J Rock Mech and Min Sci Geomech Abstr Pergamon 2 (4):389-403

David KWN, Cai W (1997) Treatingnon-compatibility problem from matter element analysis to extenics. ACM Sigice Bull 22:2-9

Dou LM, Lu CP, Mu ZL (2009) Prevention and forecasting of rock burst hazards in coal mines. Min Sci Tech (China) 19(5):585-591

Gao HX (1999) Multivariate statistical analysis application. Beijing University Press, Beijing

Gong FQ, Li XB (2007) Rockburst intensity and grading forecast based on Byesian distance discriminant analysis method. Chin J Rock Mech Eng 26(5):1012-1018

He YX (2011) Risk assessment of urban network planning in china based on the matter-element model and extension analysis. Int J Electr Power Energy Syst 33:775-782 
Heckerman D (1990) Probabilistic similarity networks. Networks 20(5):607-636

Hedley DGF (1992) Rockburst handbook for Ontario hardrock mines. Canmet SP92-1E

Hoek E, Brown ET (1980) Empirical strength criterion for rock masses. J Geotech Geoenviron Eng 106(ASCE 15715)

Hou FL, Wang MQ (1989) Criterion and prevention measures on rockburst in circular tunnel. In: Chinese Society for Rock Mechanics Engineering, Proceedings of the 2th National Rock Mechanics and Engineering. Knowledge Press. Beijing

Jensen FV, Kjærulff U, Kristiansen B (2001) The SACSO methodology for troubleshooting complex systems. AI EDAM 15(04):321-333

Kemmerer B, Mishra S, Shenoy PP (2002) Bayesian casual maps as decision aids in venture capital decision making: methods and applications. Acad Manage, pp C1-C6

Kidybiński A (1981) Bursting liability indices of coal. Int J Rock Mech Min Sci Geomech Abstr 18(4):295-304

Li J, Qian YH, Da PL (2011) The rockburst experiment usage of similar material and the mechanism of the energy releasing. Adv Mater Res 295:378-82

Liu ZJ, Li JL (2007) Comprehensive prediction method for rockburst based on fuzzy probability theory. J Yangtze River Sci Res 24:42-45

Liu D, Zou Z (2012) Water quality evaluation based on improved fuzzy matter-element method. J Environ Sci 24(7):1210

Marek U (2009) Monitoring of methane and rockburst hazards as a condition of safe coal exploitation in the mines of Kompania Weglowa SA. Proc Earth Planet Sci 1(1):54-59

Marian T (2011) Directions of changes of hard coal output technologies in Poland. Min Sci Tech (China) 21(1):1-5

Müller L (2007) Fundamentals of rock mechanics. Blackwell, London

Patynska R, Kabiesz J (2009) Scale of seismic and rock burst hazard in the Silesian companies in Poland. Min Sci Tech (China) 19(5):604-608

Ren XS, Yu XL (1999) Multivariate statistical analysis. China Statistics Press, Beijing

Russenes BF (1974) Analyses of rockburst in tunnels in valley sides. Norwegian Institute of Technology Google Scholar

Singh SP (1988) Burst energy release index. Rock Mech Rock Eng 21(2):149-155

Singh SP (1989) Classification of mine workings according to their rockburst proneness. Min Sci Tech 8(3):253-262

Srinivasan C, Arora SK, Yaji RK (1997) Use of mining and seismological parameters aspremonitors of rockbursts. Int J Rock Mech Min Sci 34:1001-1008

Stewart RD (1995) Development of seismic risk assessment method for application to rockburst-prone sites in deep-level South African gold mines. Int J Rock Mech Min Sci Geomech Abst, pp 32297A-297A(1)

Tao ZY (1987) Rockburst determination in high in-situ stress region. J People Yangtze River 25(5):32

Tan Y, Sun GZ, Guo Z (1991) A composite index K(rb) criterion for the ejection characteristics of the burst rock. Chinese J Geol 2: 193-200

Turchaninov IA, Markov GA (1981) Conditions of changing of extra-hard rock into weak rock under the influence of tectonic stresses of massifs. ISRM International Symposium

Wang YH, Li WD, Li QG, Xu Y, Tang GH (1998) Comprehensive evaluation method of fuzzy mathematics for the prediction of rockburst. Chinese J Rock Mech Eng 5(17):493-501

Wang CL, Wu $\mathrm{AX}, \mathrm{Lu} \mathrm{H}$ et al (2015) Predicting rockburst tendency based on fuzzy matter-element model. Int J Rock Mech Min Sci 75: 224-232

Weidl G, Madsen AL, Dahlquist E (2003) Object oriented Bayesian networks for industrial process operation. In: First Bayesian Applications Modeling Workshop, http://www.intel.com/ research/events/UAI03_workshop

Wiebols GA, Cook NGW (1968) An energy criterion for the strength of rock in polyaxial compression. Int J Rock Mech Min Sci Geomech Abstr 5(6):529-549

Xu LS, Wang LS, Li YL (2002) Study on mechanism and judgement of rockbursts. Chinese J Rock Soil Mech 23:300-303 
Yu HC (2009) Prediction method of rockburst proneness based on rough set and genetical gorithm. J Coal Sci Eng 15(4):367-373

Zhang JS, Lu JY, Jia YR (1991) Study on tunnel rockburst in Tian Sheng Qiao sub-hydropower station. J Hydroelectric Power (10):34-37

Zhu JR (2010) Application of fuzzy matter-element model based on coefficients of evaluation in rockburst (Natural Science Edition). J Hunan Univ Sci Technol

Open Access This chapter is licensed under the terms of the Creative Commons Attribution-NonCommercial 4.0 International License (http://creativecommons.org/licenses/by-nc/ $4.0 /$ ), which permits any noncommercial use, sharing, adaptation, distribution and reproduction in any medium or format, as long as you give appropriate credit to the original author(s) and the source, provide a link to the Creative Commons license and indicate if changes were made.

The images or other third party material in this book are included in the book's Creative Commons license, unless indicated otherwise in a credit line to the material. If material is not included in the book's Creative Commons license and your intended use is not permitted by statutory regulation or exceeds the permitted use, you will need to obtain permission directly from the copyright holder.

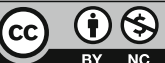




\section{Chapter 9 \\ Field Case}

Dynamic hazards predicting can be divided into single-stage predicting and large-scale mining predicting. China's metal mine production is relatively backward, a wide range of workplaces are not suitable for portable instruments into the stope for one-on-one monitoring and predicting. In addition, the monitoring results of single stope can not reflect the trend of large-scale hazard. Large-scale mining area and continuous mining area of hazard monitoring and predicting should take multi-channel instrument for positioning processing dynamic hazard (Cheng et al. 2006; Liu 2008; Li et al. 2005).

It is difficult to explain the dynamic hazards with traditional rock mechanics theory. People attempt to use the microseismic monitoring technology to predict dynamic hazard. For a long time, despite the precursor features and anomalies do not always indicate the advent of hazards. Compared with the traditional rock mechanics method, the predicting system must have a fundamental innovation in theory. In this chapter, based on the non-deterministic theory, which includes the mutation theory, synergetics, dissipative theory, fractal structure theory and artificial neural network system, the demonstration model for mine dynamic hazard monitoring was established. All of these monitoring includes: microseismic monitoring, predicting criteria models and synergetic monitoring methods. These field cases verified that these proposed aforementioned theoried and methods can predict rockburst in a reliable mode; these are meaning discoveries for predicting rockburst.

\subsection{Design of Microseismic Monitoring System in Huize Lead-Zine Mine}

In order to predict the mine dynamic hazard, microseismic monitoring technology has become the main method to realize the predicting of dynamic hazard in deep mining. The geophones can be used to detect the elastic wave emitted by the rupture 
source of rockmass using the microseismic monitoring system. The waveform can determine the coordinates and intensity of microseismic activity. Based on microseismic monitoring technology, we can get the potential micro-earthquake activity criterion and achieve predicting by micro-rupture information. Microseismic monitoring is widely used to predict the dynamic hazards in deep mining.

\subsubsection{Main Influence Factor}

Due to the particularity of deep mining, it can not rely solely on the theory to optimize the design of microseismic monitoring system. At the scene, we selected the initial selection of basic monitoring according to the mining project development and quasi-engineering layout. Then, we optimized the position of sensor within the scope of monitoring, the monitoring of target was ensured to meet the requirements and the monitoring range to the maximum.

In general, the establishment of a microseismic monitoring system must consider the following factors:

1. Monitoring object;

2. Monitoring range;

3. The objective environment of the object to be monitored;

4. Monitor the goals to be achieved;

5. Investment in monitoring systems;

6. Positioning accuracy.

In order to achieve the purpose of optimization program, we need fully consider the above factors, the design was optimized.

\subsubsection{Microseismic Monitoring System}

As shown in Fig. 2.7, the monitoring system consists of surface monitoring station, underground data exchange center (EQ), signal acquisition sensor (QS) and sensors. Due to the limitations of underground environment conditions, the layout of underground data exchange center should be considered:

(1) Choose a quiet location away from the mining area;

(2) Closing to the sensor and reducing the overall length of communication cable;

(3) Builting in a more stable rock formation to ensure the safety of data exchange center;

(4) Consider the use of underground power supply to facilitate communication with the surface monitoring station; 
(5) Consider underground ventilation, moisture and other environmental problems;

(6) Easy to transport cables and cable laying.

This microseismic monitoring system was divided into two parts: the surface and the underground parts, they were the long-term monitoring service facilities.

\subsubsection{Sensor Arrangement Design}

Regarding on the installation of microseismic monitoring system, the preliminary consideration: Considering the orebody occurrence conditions, mining methods and existing engineering layout, a QS system was be layout $1451 \mathrm{~m}$ level, two sets of QS was arranged in $1391 \mathrm{~m}$ level, a QS system was in $1331 \mathrm{~m}$ level, as shown in Fig. 2.7.

\subsection{Case Verification}

Microseismic signals contain large amounts of information. Through the analysis of precursory characteristics of microseismic signals, dynamic hazard can be predicted using these parameters.

\subsubsection{Predicting Case Based on Displacement Nephogram}

The displacement change of microseismic events varies with different periods during excavation. Many periods increase suddenly, while in some cases the increase rate is smaller, and the overall pattern is unbalanced. The cumulative displacement curves show that rock failure status. When it suddenly increases greatly, it indicates the rock failure. The phenomenon can be used as the precursor information characteristic of rock failure, which is usually formed by the accumulation of stress and deformation of rockmass. However, due to the frequent mining field blasting, drilling excavation, especially when underground mining activity is not stable, and the structure of underground mining goaf due to mining activities suddenly produce large changes, accumulation of microseismic parameters also will produce a sudden change. These changes often rebalance over a period of time, microseismic events will decrease and their strength will decrease. Therefore, when the change pattern of microseismic events is predicted by displacement accumulation curve, the underground mining activities are combined to reflect the actual situation. 
As shown in Fig. 9.2, microseismic monitoring data was from April 14th, 2009 to 30th, it showed that the distribution characteristics of microseismic events using microseismic monitoring system XQuery software.

The gradual extraction of orebody was shown in Fig. 9.1, Activity distribution of induced microseismic events were activity in 6 layers and two plates in the middle section of $1391 \mathrm{~m}$ in April 2009, The red line represents the exploration line and typical fault distribution, the orebodies were included in the blue line frame as indicated by the key monitoring areas. The intermediate microseismic monitoring events indicated the displacement characteristics of rockmass, and the color ranges from light blue to red indicated displacement, microseismic events of different colors indicated the development process of rock displacement induced by microseismic events.

As shown in the displacement nephogram of Fig. 9.1, it could be found that the rockmass displacement was mainly between the 52 lines and the 54 lines, and there was a trend of development near the fault. Based on the microseismic monitoring data, it showed that the development of rockmass displacement was mainly concentrated near the fault. The stope No. 3, which was in the middle two layered panel of $1391 \mathrm{~m}$, produced a larger displacement in the vicinity of line 52 (red circle) $4.57 \mathrm{E}-4 \mathrm{~m}$. With the development of mining, the displacement increased gradually to $4.92 \mathrm{E}-4 \mathrm{~m}$, the change was small, but the range of deformation increased, especially in front of fault.

Through the displacement of microseismic events was analyzed and compared in different mining area, it could draw the displacement range and development trend occurred in a certain period of time of microseismic events, and obtained the risk of dynamic hazard caused by mining.
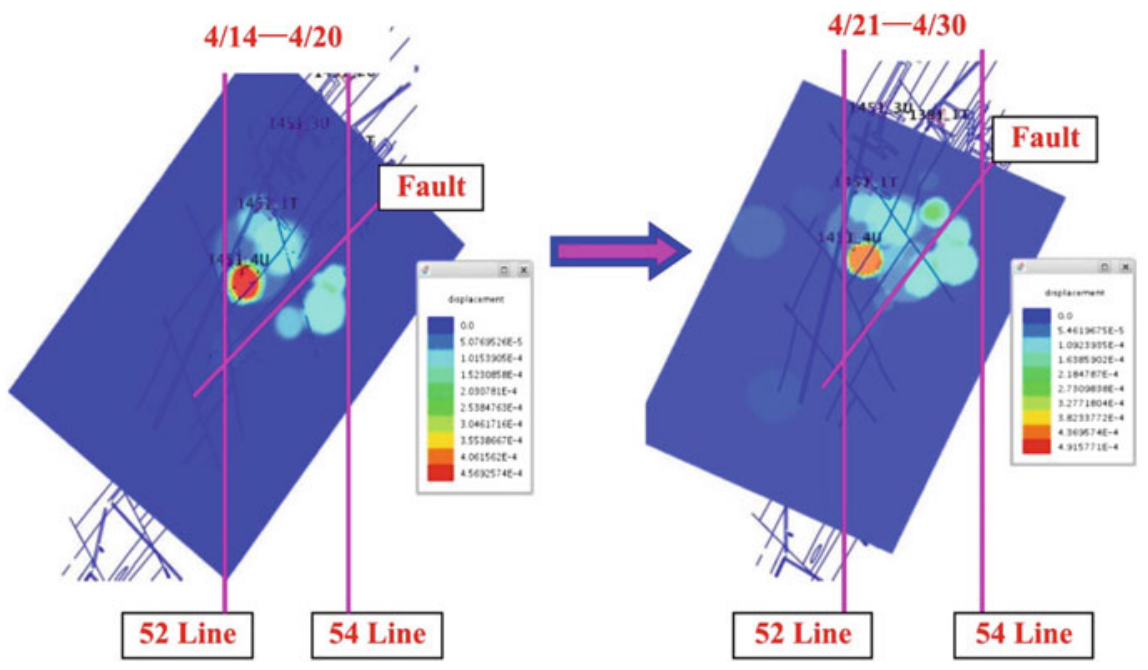

Fig. 9.1 Displacement nephogram 


\subsubsection{Predicting Case Based on Apparent Stress}

Because mining disturbed the stress status of original rock, microseismic events were caused by rockmass failure, shear stress. Before the failure of rockmass, shear stress showed the stress drop of hypocenter. According to quantitative seismology theory, stress level and stress drop $\Delta \sigma$ of earthquake source rock could be expressed by seismic apparent stress $\sigma_{A}$. The deformation produced by earthquake source failure could be expressed by displacement $X(x, y, z)$. Therefore, it was known that stress-strain status of rockmass could be obtained by the spatial location, occurrence time $t$, focal sight stress $\sigma_{A}$ and displacement $u$ of microseismic events. That is to say, in a certain period of time $\Delta t$ and space volume $\Delta V$ of rockmass, microseismic events could be used to describe the spatial distribution of stress and deformation in rockmass.

According to the existing condition of $8 \#$ ore body and the characteristics of mining engineering layout, the stress distribution at different elevation levels can provide a simple and direct description of microseismic activity in the mining area, a series of apparent stress distributions at different time periods were plotted in the visual stress analysis diagram. As shown in Fig. 9.2, many colors represented the apparent stress status at different times. The maximum apparent stress was $4.93 \mathrm{~Pa}$ in the fault position. With the recovery, the central region was not through the two segment stress and apparent stress, but stress concentration area in the near fault. As the right view is showed, the stress concentration area was also expanding, but the apparent stress value changed little. The occurrence, concentration, development and coalescence of apparent stress showed that large scale mining engineering caused obvious stress concentration or deformation concentration. Therefore, with

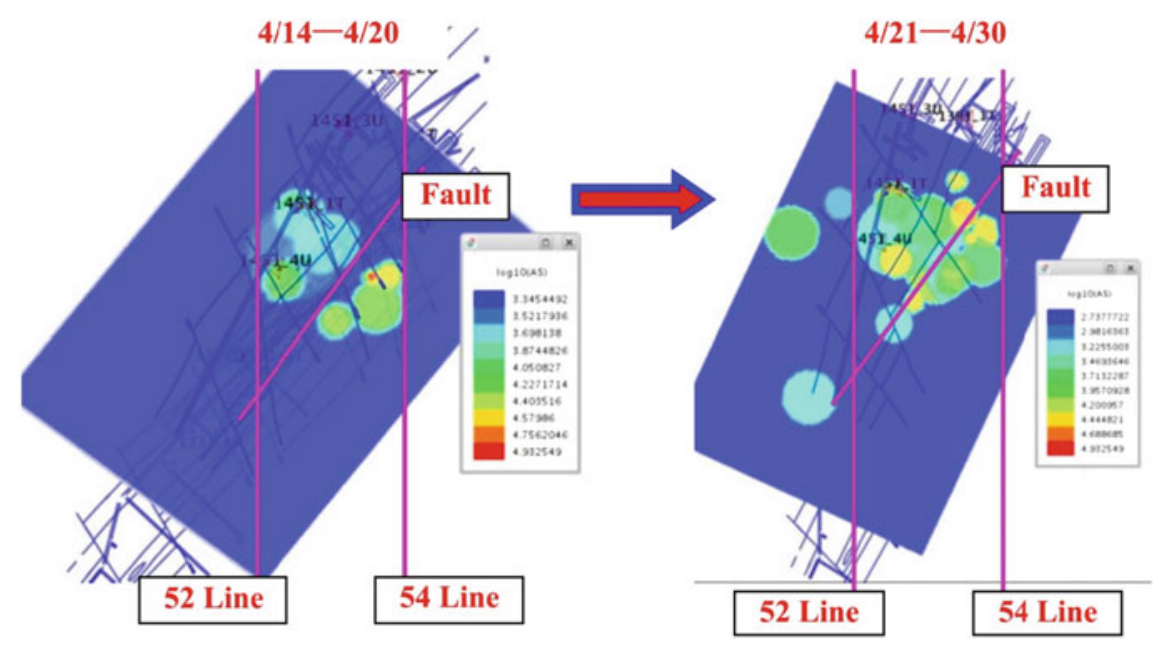

Fig. 9.2 Visual stress variation nephogram of microseismic monitoring events 
the development of mining, using the development trend of apparent stress of rockmass, monitoring can be strengthened in this area to ensure safe production.

\subsection{Predicting Key Points Identification of Dynamic Hazard}

\subsubsection{Dynamic Hazard Predicting Using Routine Monitoring}

A case to verify the method of Tangent Modulus (TM) identification of dynamic hazard predicting critical point, which changes after the point $B$ tangent modulus to determine the damage degree of rock deformation, so as to identify the key points to realize dynamic hazard predicting.

It is a very important method to obtain reliable physical parameters of rockmass using field monitoring instrument. According to the field engineering environment and mining plan, the stress and displacement monitoring, which was in 8\# ore body $1331,1345,1369$ and $1391 \mathrm{~m}$, were carried out in four stages, and the $1369 \mathrm{~m}$ monitoring data were selected to be analysis. The surrounding rockmass was $C_{1 b}$, and multi-point displacement meter were installed to monitor the displacement change in 1369-1 and 1369-4 bore holes; a bore hole stress gauge was installed in bore holes 1369-2, 1369-3 and 1369-9, the change of maximum principal stress was used to monitor in 1369-3 (the direction of ore body), and the tendency of stress changes was monitored in 1369-9 along with the direction of orebody; multi-point displacement meter was equipped with drilling 1369-7 and 1369-8 to monitor the deformation of rockmass caused by the minimum principal stress.

Through the stress and deformation of $1369 \mathrm{~m}$ segment rock was monitored, it was shown that the stress increment was only 1.2 MPa. With the gradual progress of mining engineering, the stress increased gradually, and the increase of displacement was unconspicuous in a long time. As shown in Fig. 9.3, until the point $B$ in the elastic stage, TM remained nearly straight up, reached at $1,243 \mathrm{MPa}$, the stress increment caused by the energy accumulation increased gradually the inner rockmass. As shown in Fig. 9.4, stress increment value increased slowly after the point $\mathrm{B}$, the displacement changed greatly, TM decreased rapidly, and the change tended to be gradual only $880 \mathrm{MPa}$. It showed that rockmass passed through the yielding point, and rockmass was gradually developing to the instability direction. Until the failure, the average modulus of rock was $610 \mathrm{MPa}$. Therefore, the $B$ point was defined as a predicting point. From the apparent observation of rockmass, micro-cracks expanded and failure, energy was released, the bearing capacity of rockmass decreased gradually, and the failure strain rate further speeded up. The instrument could not be monitored because of local damage in October 28th, 2010. In conclusion, it was reasonable to take yield point $B$ as a key predicting 
Fig. 9.3 $1369 \mathrm{~m}$ stress strain curve. From Wang (2013)

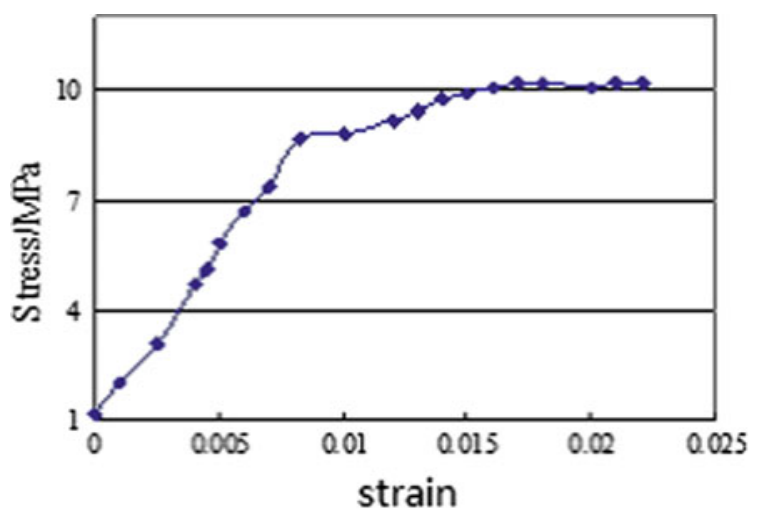

Fig. 9.4 Identification of predicting key points based on TM. From Wang (2013)

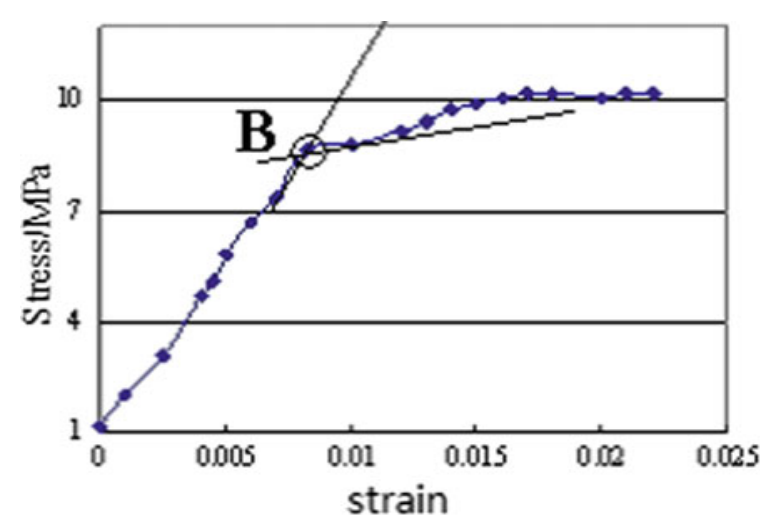

point. If the stress passed through the yield point $B$, but the stress may increased slowly and the deformation slowed down. Therefore, rockmass would have a long time to reach the peak strength, and then the rockmass would be failure. TM at the peak strength was 0 , TM divided into several segments which was the yield point to the peak intensity for predicting rock failure.

\subsubsection{Predicting Key Point Identification Using CAV and MS Events Activity}

MS activity rate and Cumulative apparent volume (CAV) of low-energy and small-magnitude MS events had sharply increased, as shown in Fig. 9.5. When the high-energy and high-magnitude MS events increased dramatically, rockmass had passed through the elastic stage and began to enter the yield point ( $12 \mathrm{~h} 00$ until $24 \mathrm{~h} 00$ on September 10th, 2009). The MS events activity rate peaked between 


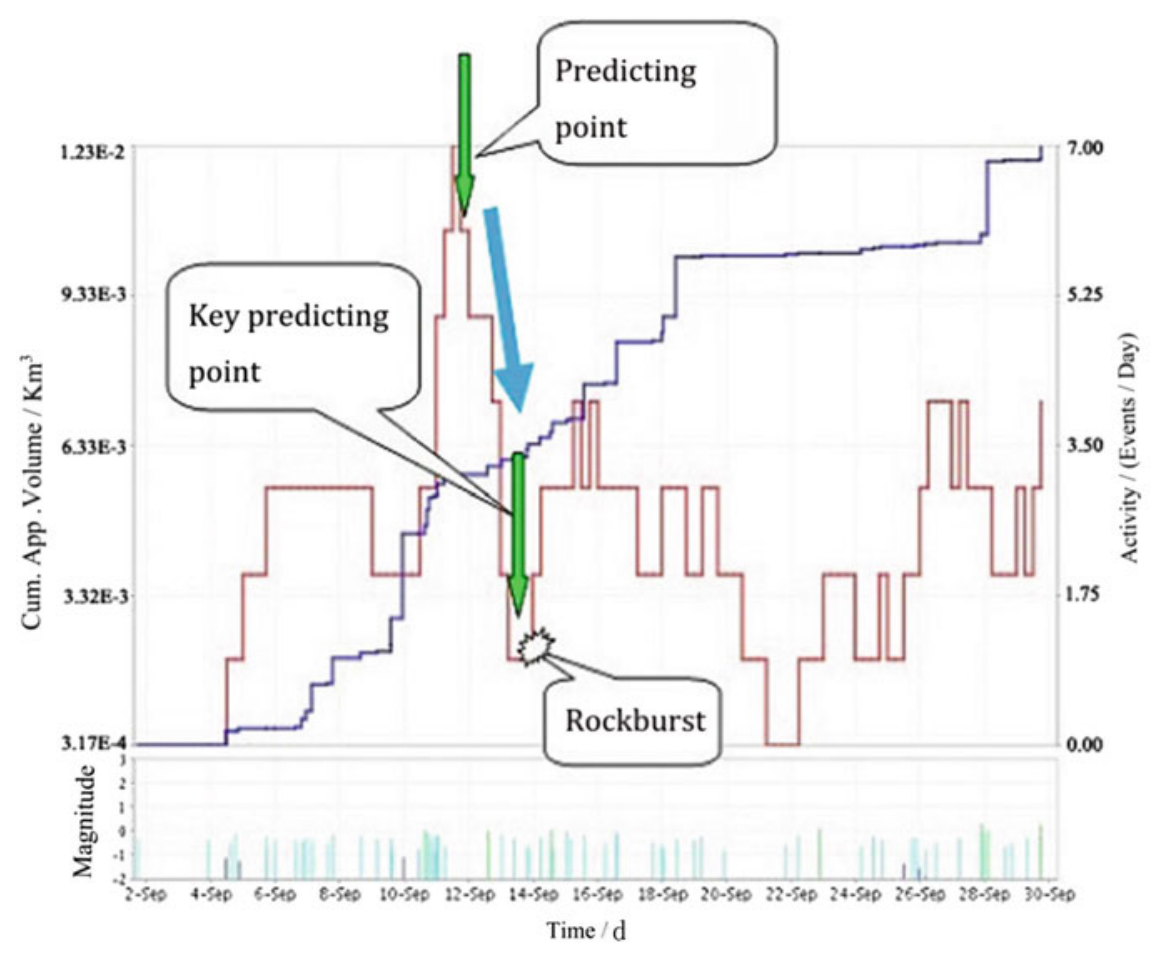

Fig. 9.5 Indentifying the predicting key point with microseismic events activity rate and CAV. After Wang (2014)

$14 \mathrm{~h} 00$ and $18 \mathrm{~h} 00$ on September 11st, 2009 and then rapidly declined. This chapter defined the predicting point of rock failure as from $22 \mathrm{~h} 00$ until $24 \mathrm{~h} 00$ on September 11st, 2009. When MS events entered a relatively quiet period accompanied by a CAV surge from $0 \mathrm{~h} 00$ until $4 \mathrm{~h} 00$ on September 13rd, 2009, this point was interpreted as a key predicting point. At 0 h 00 on September 14th, 2009, roadway collapse accidents occurred in mine road 2, slice 6, sublevel 1369, level $1331 \mathrm{~m}$ (argillaceous limestone, approximately $100 \mathrm{t}$ ), and the activity rate of MS events and CAV of seismic source simultaneously increased sharply, as shown in Fig. 9.5. Rock failure was located with MS monitoring system in Fig. 9.6, and occurred in field in Fig. 9.7. The results showed that stress entered the predicting point of rockburst with the sharp increase in the activity of MS events and CAV. With increasing stress, the beginning of quiet period of MS events was the predicting key point of rockburst. After the quiet period, MS events were active and CAV increased sharply, rockburst occurred.

It should be noted that the relationship between rockmass failure and a decrease in MS event activity rate or increase in the CAV was an unnecessary but sufficient condition. The decreased $\mathrm{AE}$ events might be the stage of original crack compaction, so rockburst might not occur after predicting. Therefore, not all decreased 


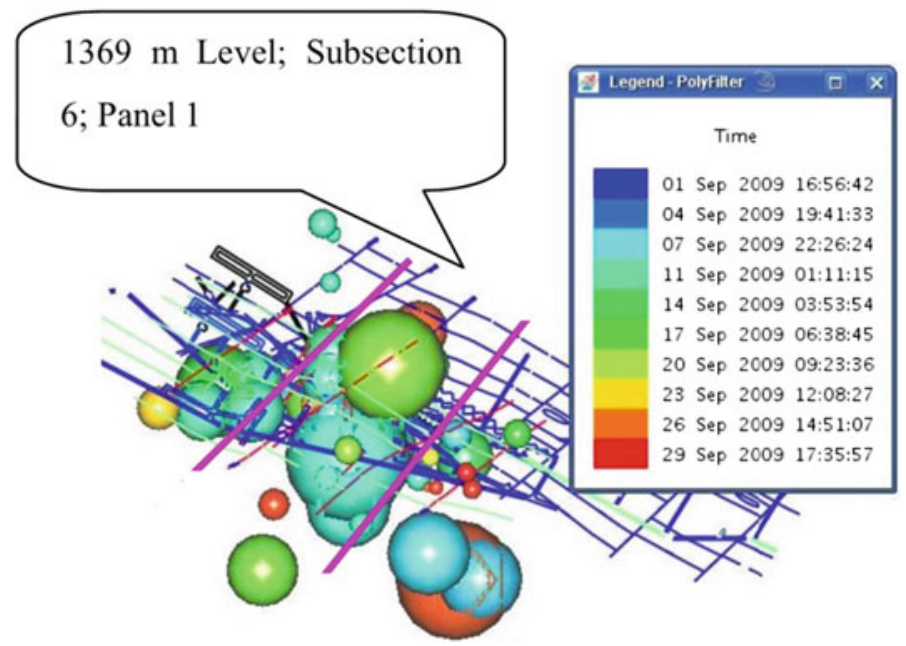

Fig. 9.6 Distribution of microseismic events. From Wang (2014)

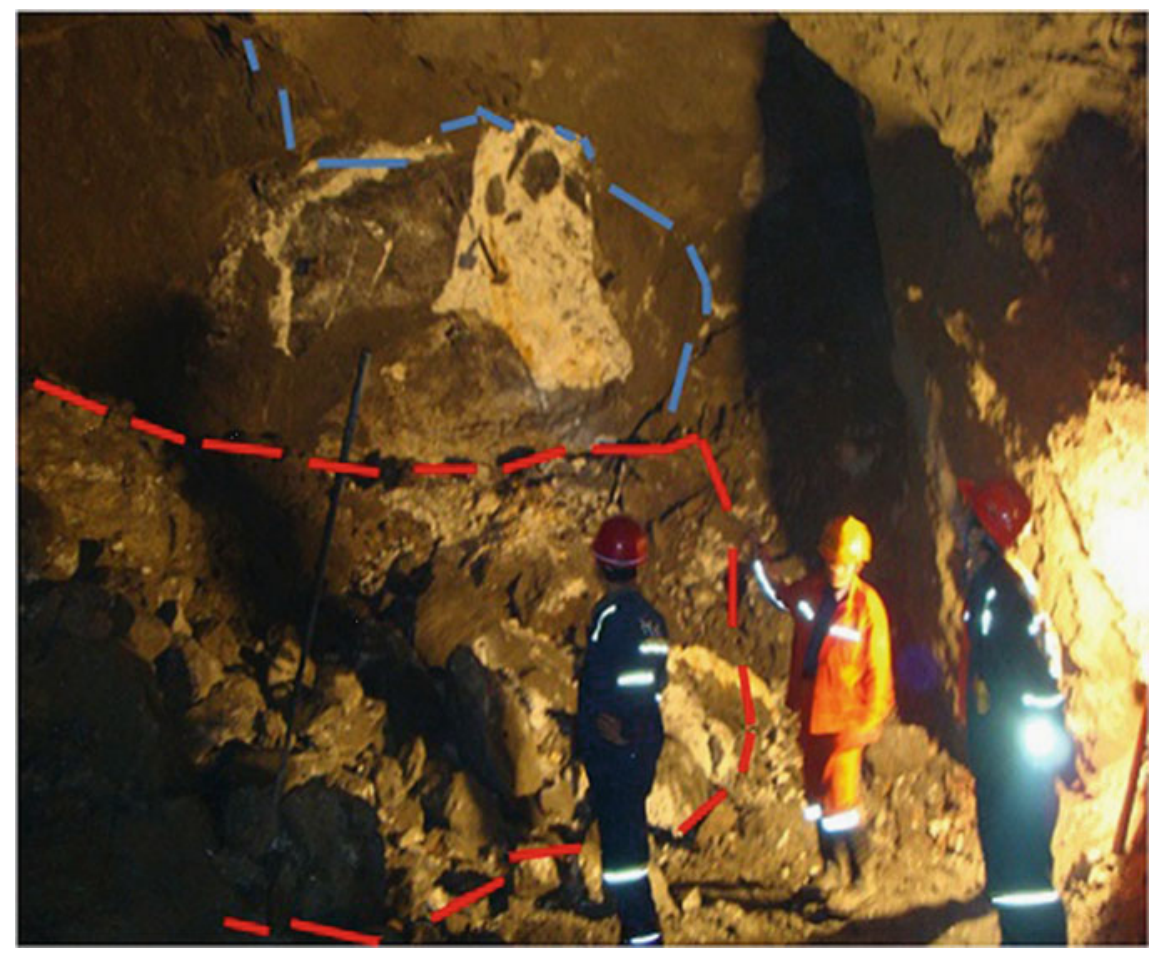

Fig. 9.7 Collapse in 2\# tunnel. With kind permission from "PERGAMON-ELSEVIER SCIENCE LTD”: Fig. 5, Wang (2014). All rights reserved 
in MS events activities or increased in the CAV indicated that rockmass was about to incur inevitable instability. However, it could play a key role in predicting rockburst. In the field, MS monitoring was using to predict rockburst in deep mining. Advanced monitoring was implemented by MS throughout the entire process of rockmass deformation. The activity rate and CAV of low-energy and small-magnitude MS events had sharply increased, as shown in Fig. 9.5. Rockburst was located with MS monitoring system in Fig. 9.6, and occurred in field in Fig. 9.7. The results showed that stress entered the predicting point of rockmass instability with the sharp increasement in the activity of MS events and CAV. With increasing stress, the beginning of quiet period of MS events was the predicting key point of rockburst. After the quiet period, MS events were active and CAV increased sharply, rockburst occurred.

\subsection{Predicting Model of Rockburst Based on Bayesian Theory}

\subsubsection{Training Samples}

We have been engaged in field tests and laboratory research on underground engineering dynamic hazard prevention for 16 years. It was determined that rockburst grades are approximately power-law distribution. That is, strong and very weak rockbursts are relatively less frequent, while moderate and weak rockbursts account for the majority of cases. The Bayesian discriminant model is based on assuming a normal distribution for the input variables, as well. The geological description, in situ stress measurement, and laboratory testing procedures were described previously. Experimental samples were randomly selected from a large data set for training the Bayesian model. The basic parameters of training samples and associated rockburst grades are listed in Table 9.1.

\subsubsection{Predicting Rockburst Tendency Using a Bayesian Model}

In this chapter, the critical factors, such as $R_{b}\left(X_{1}\right), R_{\theta}\left(X_{2}\right)$ and $W_{\text {et }}\left(X_{3}\right)$, were used as the basic parameters for predicting rockburst risk using a Bayesian model. 
Table 9.1 Critical factors of training samples

\begin{tabular}{l|l|l|l|l|l|l|l}
\hline No. & $\sigma_{c}$ & $\sigma_{t}$ & $\sigma_{\theta}$ & $R_{b}$ & $R_{\theta}$ & $W_{\text {et }}$ & Actual rockburst grades \\
\hline 1 & 100.08 & 8.46 & 60.70 & 11.83 & 0.61 & 6.56 & Strong \\
\hline 2 & 140.68 & 10.89 & 78.40 & 12.92 & 0.56 & 5.52 & Strong \\
\hline 3 & 250.54 & 9.85 & 97.55 & 25.44 & 0.39 & 8.60 & Strong \\
\hline 4 & 88.77 & 3.74 & 30.53 & 23.74 & 0.34 & 6.23 & Medium \\
\hline 5 & 180.44 & 8.15 & 67.36 & 22.14 & 0.37 & 5.00 & Medium \\
\hline 6 & 236.80 & 8.37 & 109.32 & 28.29 & 0.46 & 4.65 & Medium \\
\hline 7 & 120.38 & 6.53 & 98.68 & 18.43 & 0.82 & 3.50 & Medium \\
\hline 8 & 130.14 & 6.86 & 55.40 & 18.97 & 0.43 & 4.64 & Medium \\
\hline 9 & 180.13 & 6.33 & 65.42 & 28.46 & 0.36 & 3.45 & Weak \\
\hline 10 & 64.24 & 2.14 & 18.15 & 30.02 & 0.28 & 4.97 & Weak \\
\hline 11 & 82.46 & 4.20 & 21.71 & 19.63 & 0.26 & 2.56 & Weak \\
\hline 12 & 89.33 & 3.33 & 27.56 & 26.83 & 0.31 & 3.32 & Weak \\
\hline 13 & 120.69 & 5.41 & 30.22 & 22.31 & 0.25 & 4.34 & Weak \\
\hline 14 & 195.53 & 7.10 & 42.60 & 27.54 & 0.22 & 5.55 & Weak \\
\hline 15 & 115.50 & 3.52 & 11.62 & 32.81 & 0.10 & 2.70 & No \\
\hline 16 & 150.93 & 5.42 & 34.21 & 27.85 & 0.23 & 2.80 & No \\
\hline 18 & 178.96 & 4.37 & 18.80 & 40.95 & 0.11 & 1.46 & No \\
\hline
\end{tabular}

The classification categories of rockburst tendency were strong $\left(G_{1}\right)$, moderate $\left(G_{2}\right)$, weak $\left(G_{3}\right)$, and no rockburst $\left(G_{4}\right)$. In other words, all of basic parameters were included in the three-dimensional matrix $G=\left(X_{1}, X_{2}, X_{3}\right)^{T}$, which formed the dataset of Bayesian model. These results were calculated as follows:

According to those selected training samples, the empirical probability is

$$
p_{1}=\frac{3}{18}, p_{2}=\frac{5}{18}, p_{3}=\frac{6}{18}, p_{4}=\frac{4}{18}
$$

The mean values of variable categories are

$$
\begin{aligned}
& \mu_{1}\left(X_{1}^{(1)}, X_{2}^{(1)}, X_{3}^{(1)}\right)^{T}=(16.73,0.52,6.89) \\
& \mu_{2}\left(X_{1}^{(2)}, X_{2}^{(2)}, X_{3}^{(2)}\right)^{T}=(22.31,0.48,4.80) \\
& \mu_{3}\left(X_{1}^{(3)}, X_{2}^{(3)}, X_{3}^{(3)}\right)^{T}=(25.80,0.28,4.03) \\
& \mu_{4}\left(X_{1}^{(4)}, X_{2}^{(4)}, X_{3}^{(4)}\right)^{T}=(29.55,0.15,2.57)
\end{aligned}
$$


The matrix of mean values can be expressed as

$$
\bar{X}=\left[\begin{array}{cccc}
16.73 & 22.31 & 25.80 & 29.55 \\
0.52 & 0.48 & 0.28 & 0.15 \\
6.89 & 4.80 & 4.03 & 2.57
\end{array}\right]
$$

The covariance matrixes of sample categories were

$$
\begin{aligned}
& S_{1}^{2}=\left[\begin{array}{ccc}
57.16 & -0.85 & 10.86 \\
-0.85 & 0.01 & -0.15 \\
10.86 & -0.15 & 2.45
\end{array}\right] \\
& S_{2}^{2}=\left[\begin{array}{ccc}
16.00 & -0.36 & 1.67 \\
-0.36 & 0.04 & -0.16 \\
1.67 & -0.16 & 0.96
\end{array}\right] \\
& S_{3}^{2}=\left[\begin{array}{ccc}
15.83 & 0.07 & 2.46 \\
0.07 & 0.01 & -0.03 \\
2.46 & -0.03 & 1.26
\end{array}\right] \\
& S_{4}^{2}=\left[\begin{array}{ccc}
103.77 & -0.36 & -7.36 \\
-0.36 & 0.01 & 0.03 \\
-7.36 & 0.03 & 0.61
\end{array}\right]
\end{aligned}
$$

The covariance matrix of sample population was calculated as follow:

$$
\Sigma=\left[\begin{array}{ccc}
40.63 & -0.27 & 1.33 \\
-0.27 & 0.01 & -0.07 \\
1.33 & -0.07 & 1.20
\end{array}\right]
$$

while its inverse is

$$
\Sigma^{-1}=\left[\begin{array}{ccc}
0.03 & 0.56 & 0.01 \\
0.56 & 113.79 & 6.25 \\
0.01 & 6.25 & 1.20
\end{array}\right]
$$

The discriminants for sample categories were obtained:

$$
\begin{aligned}
& \omega_{1}\left(X_{1}, X_{2}, X_{3}\right)=0.78 x_{1}+111.41 x_{2}+11.58 x_{3}-77.12 \\
& \omega_{2}\left(X_{1}, X_{2}, X_{3}\right)=0.92 x_{1}+97.77 x_{2}+8.88 x_{3}-56.56 \\
& \omega_{3}\left(X_{1}, X_{2}, X_{3}\right)=0.90 x_{1}+71.71 x_{2}+6.68 x_{3}-36.26 \\
& \omega_{4}\left(X_{1}, X_{2}, X_{3}\right)=0.93 x_{1}+49.87 x_{2}+4.11 x_{3}-24.28
\end{aligned}
$$

Each group of sample data was brought into each discriminant function to obtain the corresponding posterior probability. 


\subsubsection{Verifying Accuracy of Bayesian Model}

The research goal is to establish a more reliable model for predicting rockburst risk. Therefore, we introduced a multivariable Bayesian model using data of both lab and field. As shown in Table 9.2 and Fig. 9.8, the final classifications and posterior probabilities were calculated, demonstrating a high rate of accuracy.

Based on the original data, the results of predicting rockburst using the existing methods were compared. Significant differences were observed between the results of new method and the previous ones, even for a single sample, as shown in Table 9.2. With regard to predicting the actual rockburst grades, the accuracy rates of $R_{b}, R_{\theta}$ and $W_{\text {et }}$ in isolation were 61,72 and $56 \%$, respectively. However, the multivariable Bayesian model was found to be significantly more reliable, with an accuracy rate of $94 \%$. Only sample No. 11 experienced a relatively small error. These results were consistent with the notion that the Bayesian model could achieve more reliable predictions of rockburst risk.

In fact, there are many important factors that influence rockburst. Only a few factors were considered in the previous prediction methods. Rock brittleness index $\left(R_{b}\right)$ was only considered the uniaxial compression strength and tensile strength; the stress of surrounding rockmass was not included in this model. Russenses's method $\left(R_{\theta}\right)$ deals with the principal stress of surrounding rockmass and uniaxial

Table 9.2 Posterior probability and return test

\begin{tabular}{l|c|c|l|l|l|l}
\hline \multirow{2}{*}{ No. } & \multicolumn{3}{|l}{ Discriminants } & \multicolumn{2}{l}{ Classified results } & Backward possibility (\%) \\
\cline { 2 - 5 } & W1 & W 2 & W3 & W4 & & \\
\hline 1 & $\mathbf{7 5 . 7 2}$ & 71.84 & 61.72 & 43.94 & Strong & 98.78 \\
\hline 2 & $\mathbf{5 9 . 0 5}$ & 58.80 & 52.22 & 38.22 & Strong & 68.10 \\
\hline 3 & $\mathbf{8 5 . 8 3}$ & 81.22 & 72.03 & 54.15 & Strong & 99.41 \\
\hline 4 & 51.99 & $\mathbf{5 4 . 1 8}$ & 51.41 & 40.56 & Medium & 80.73 \\
\hline 5 & 39.76 & $\mathbf{4 4 . 6 7}$ & 43.86 & 35.48 & Medium & 72.29 \\
\hline 6 & 50.38 & $\mathbf{5 5 . 8 5}$ & 53.40 & 44.17 & Medium & 92.73 \\
\hline 7 & 69.22 & $\mathbf{7 1 . 6 0}$ & 62.51 & 48.13 & Medium & 86.62 \\
\hline 8 & 38.94 & $\mathbf{4 3 . 6 8}$ & 42.36 & 33.67 & Medium & 80.92 \\
\hline 9 & 25.64 & 35.73 & $\mathbf{3 8 . 4 6}$ & 34.48 & Weak & 90.47 \\
\hline 10 & 35.49 & 42.77 & $\mathbf{4 4 . 2 4}$ & 38.16 & Weak & 78.26 \\
\hline 11 & -2.72 & 9.95 & 17.41 & $\mathbf{1 7 . 6 3}$ & No & 65.21 \\
\hline 12 & 16.77 & 27.73 & $\mathbf{3 2 . 2 1}$ & 29.70 & Weak & 88.05 \\
\hline 13 & 18.56 & 26.95 & $\mathbf{3 0 . 7 9}$ & 26.80 & Weak & 94.91 \\
\hline 14 & 33.05 & 63.96 & $\mathbf{4 1 . 2 5}$ & 35.01 & Weak & 94.54 \\
\hline 15 & -8.88 & 7.40 & 18.55 & $\mathbf{2 2 . 3 5}$ & No & 98.53 \\
\hline 16 & 2.42 & 16.05 & 23.78 & $\mathbf{2 4 . 4 3}$ & No & 74.08 \\
\hline 17 & -16.36 & 4.31 & 17.91 & $\mathbf{2 5 . 0 4}$ & No & 99.95 \\
\hline 18 & -6.79 & 4.73 & 13.02 & $\mathbf{1 3 . 2 6}$ & No & 65.71 \\
\hline
\end{tabular}

Note The Bold words are the maximum value of the discriminants, which indicates that the sample belongs to that classification. 

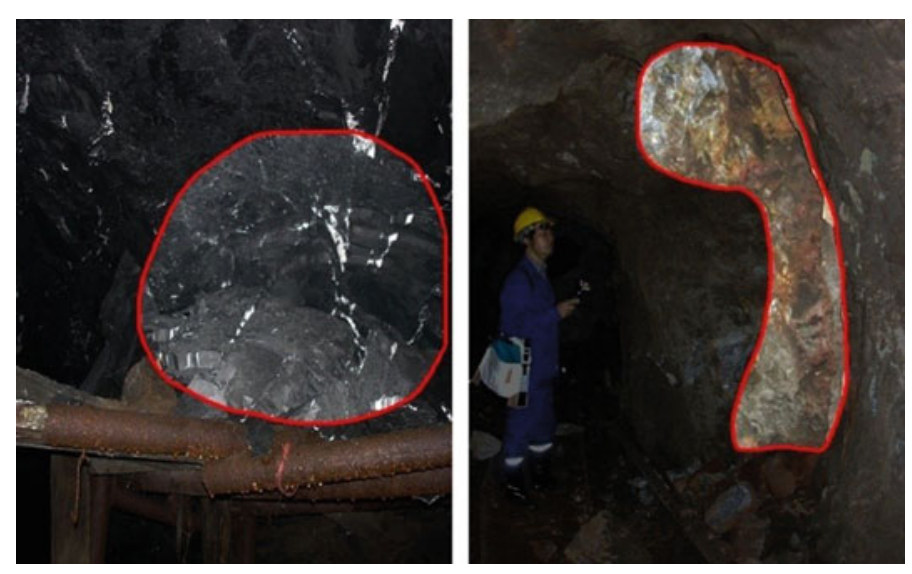

Fig. 9.8 Rockburst in underground mining

compression strength. However, it did not account for the accumulated energy and rigidity of surrounding rockmass. Finally, Kidybinski's method $\left(W_{\mathrm{et}}\right)$ measured the rock capacity to store and release elastic energy, while many other factors were ignored. Obviously, there were limitations in the traditional methods for predicting rockbursts. Because rockburst was induced by multiple factors, considering a single factor alone inevitably leaded to imprecise results. Conversely, our model was benefitted from integrating the variables used in traditional methods, which could be input into the Bayesian model. In this chapter, $R_{b}, R_{\theta}$ and $W_{\text {et }}$ were used as the critical factors in proposed model, plus the surrounding rockmass stress $\sigma_{1}$ and $\sigma_{\theta}$, and rock strength $\sigma_{c}$ and $\sigma_{t}$.

As shown in Table 9.3, the results were inconsistent for all samples using the previous methods, except for \#1, 2, 8, and 16. Moreover, at least one method gave the incorrect rockburst grade for all samples besides \#1, 2 and 8. On the other hand, the proposed model always returned the correct grade, except for sample 16.

The traditional methods were also limited in that they cannot differentiate the relative importance of different variables. In addition, it was not always reasonable to update the variables to coordinate the field data. A certain types of artificial intelligence, such as the neural network models, could consider many factors simultaneously; these methods did not address the empirical probabilities and posterior probabilities in their calculations. However, a Bayesian model could overcome these shortcomings to provide an ideal method for rockburst tendency prediction.

Because of limited availability of sample data, a self-validation process was implemented on the predictions from the new model. However, our results demonstrate that the new model is a significant improvement over existing methods, and can be implemented for predicting the reliable rockburst tendency. 
Table 9.3 Comparison of predicted results

\begin{tabular}{l|l|l|l|l|l}
\hline No. & $\begin{array}{l}\text { Rock } \\
\text { brittleness } \\
\text { index }\left(R_{b}\right)\end{array}$ & $\begin{array}{l}\text { Russenses's } \\
\text { method }\left(R_{\theta}\right)\end{array}$ & $\begin{array}{l}\text { Kidybinski's } \\
\text { method }\left(W_{\mathrm{et}}\right)\end{array}$ & $\begin{array}{l}\text { Proposed } \\
\text { model }\end{array}$ & $\begin{array}{l}\text { Actual } \\
\text { rockburst } \\
\text { grades }\end{array}$ \\
\hline $\mathbf{1}$ & Strong & Strong & Strong & Strong & Strong \\
\hline $\mathbf{2}$ & Strong & Strong & Strong & Strong & Strong \\
\hline $\mathbf{3}$ & Moderate & Moderate & Strong & Strong & Strong \\
\hline $\mathbf{4}$ & Moderate & Moderate & Strong & Moderate & Moderate \\
\hline $\mathbf{5}$ & Moderate & Moderate & Strong & Moderate & Moderate \\
\hline $\mathbf{6}$ & Weak & Moderate & Moderate & Moderate & Moderate \\
\hline $\mathbf{7}$ & Moderate & Strong & Moderate & Moderate & Moderate \\
\hline $\mathbf{8}$ & Moderate & Moderate & Moderate & Moderate & Moderate \\
\hline 9 & Weak & Moderate & Moderate & Weak & Weak \\
\hline 10 & Weak & Weak & Moderate & Weak & Weak \\
\hline 11 & Moderate & Weak & Weak & None & Weak \\
\hline 12 & Moderate & Moderate & Weak & Weak & Weak \\
\hline 13 & Moderate & Weak & Moderate & Weak & Weak \\
\hline 14 & Weak & Weak & Strong & Weak & Weak \\
\hline 15 & Weak & None & Weak & None & None \\
\hline $\mathbf{1 6}$ & Weak & Weak & Weak & None & None \\
\hline 17 & Weak & None & None & None & None \\
\hline 18 & Moderate & None & Weak & None & None \\
\hline
\end{tabular}

Note The Bold and Italic words are the same as results using the different methods; The Bold words are different results by diverse methods.

\subsection{Predicting Model of Rockburst Based on Fuzzy Matter Element Theory}

\subsubsection{Testing and Field Condition}

\subsubsection{Mining Conditions and Method}

The $10 \#$ ore body in the Huize Lead-Zinc Mine is one of largest $\mathrm{Pb}-\mathrm{Zn}$ deposits in China, and it is rich in poly metallic deposits including plumbum, zinc, and germanium. There are three ore bodies in the mine; they are 6\#, $8 \#$ and $10 \#$. The $6 \#$ ore body is located on upside the $8 \#$ ore body. The $6 \#$ and $8 \#$ ore body are close. However, the $10 \#$ ore body is located on the side of $8 \#$ ore body. The $10 \#$ ore body is the last one. In this chapter, the purpose of study plan is the predicting the rockburst for $10 \#$ ore body in this mine. The geological description was described in the document mining geometry and sequence in the volume (Wang et al. 2009; He and Wang 2010).

The ore grade of lead and zinc reaches $28 \%$. The ore body consisted of sulfided and oxidized minerals. The surrounding rockmass is composed of four types of rock strata, which are lime stone $\left(C_{3 m}\right)$, hanging wall lime stone $\left(C_{2 w}\right)$, dolomite $\left(C_{1 b}\right)$ 

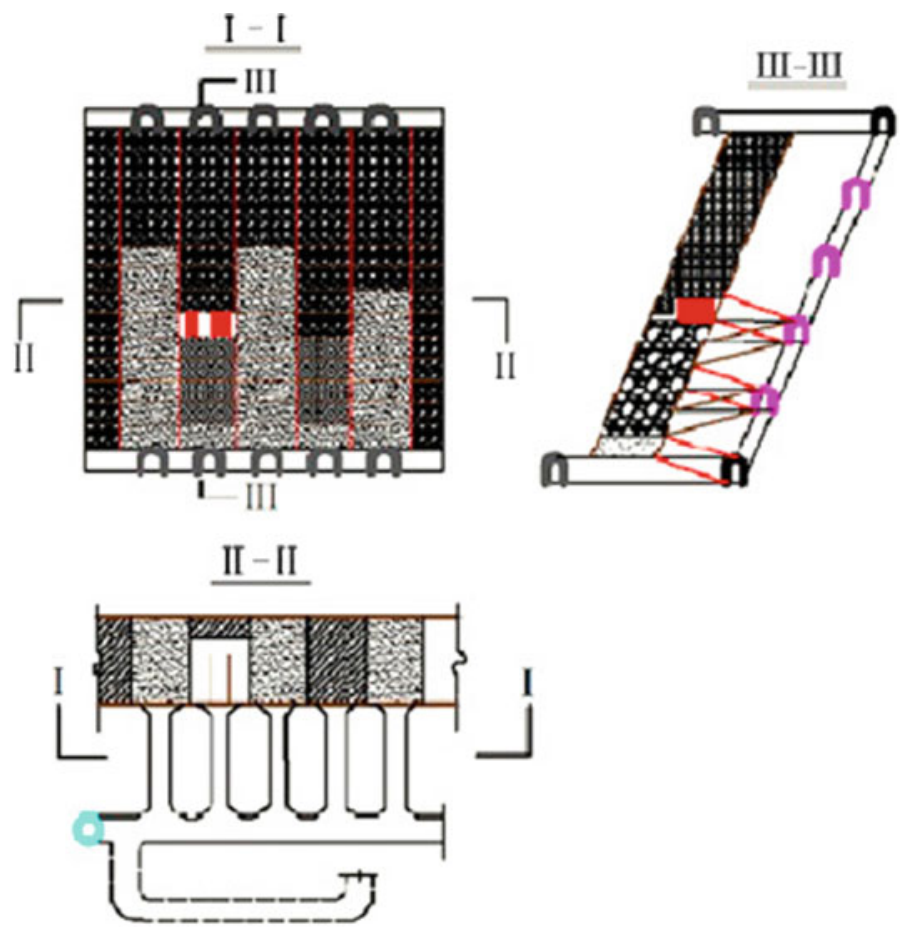

Fig. 9.9 Paste filling mining method of upward horizontal slice. With kind permission from "PERGAMON-ELSEVIER SCIENCE LTD": Fig. 1, Wang (2014). All rights reserved

and foot wall lime stone $\left(C_{1 d}\right)$. In $10 \#$ ore body, the working conditions are very complicated because of large depth, ample water, and fractured ore bodies. The deepest minable ore body is buried at a depth of more than $1500 \mathrm{~m}$, which is now one of the deepest metal mines in China. This deep mining area is located in the eastern Yun-Gui plateaucany on area with very high virgin stress. With the increasing mining depths, the occurrence of rockburst increases and possesses greater safety threats to mining production. Adopting on the upward horizontal slice paste filling mining method in this mine, it was divided into two steps. As shown in Fig. 9.9. The mining sequence is from top to bottom. Nowadays, the goafs were immediately back filled with paste material. The length of $10 \#$ ore body strike and dip was 300 and $60 \mathrm{~m}$, and the strike is $\mathrm{N} 26^{\circ} \mathrm{E}$. The mine is mining the level 1331-1451 m level, level height was $60 \mathrm{~m}$; sublevel height was $12 \mathrm{~m}$, and layer height was $3 \mathrm{~m}$. Stopes were vertically designed with the strike of ore body. Mining sequence is from $1451 \mathrm{~m}$ level to $1331 \mathrm{~m}$ level. The width of stope and pillar was $5 \mathrm{~m}$ or $6 \mathrm{~m}$ two schemes, the length was equal to the ore body thickness. Room was first stoped, and then room was filled with pasting. Pillar was stoped after the adjacent rooms were filled. 


\subsubsection{Laboratory Testing}

In this testing, the physic-mechanic parameters of sulfide and oxidized ore body, disk lime stone $\left(C_{3 m}\right)$, hanging wall lime stone $\left(C_{2 w}\right)$, dolomite $\left(C_{1 b}\right)$ and foot wall lime stone $\left(C_{1 d}\right)$ samples were used testing, all tested samples were made and processed to standard cylindrical samples with $50 \mathrm{~m}$ mindiameter and $100 \mathrm{~m}$ from 1391 level. The elasticity modulus $(E)$, Poisson's ratio $(\mu)$, uniaxial tensile strength $\left(R_{t}\right)$ and uniaxial compressive strength $\left(R_{c}\right)$ were conducted on rock mechanics testing system (MTS815), a computer controlled, servo hydraulic compression machine. The testing system was a Windows based platform with visual control operating software, which could record the current time, load, stress, displacement, strain value, load-displacement curve and stress-strain curve, etc. The equal-displacement loading was selected as the control mode in the test. The specimen was loaded at a constant loading rate of $2 \times 10^{-3} \mathrm{~mm} / \mathrm{s}$ until the specimen fails. $R_{t}$ was calculated using the Brazil testing method, while $V_{p}$ and $V_{p m}$ were obtained with the CE-9201 rock engineering testing machine, where $V_{p}$ was the longitudinal wave velocity of sample, and $V_{p m}$ was the longitudinal wave velocity of rockmass. The tested physi-mechanical parameters of sulfide and oxidized ore body, disk lime stone $\left(C_{3 m}\right)$, hanging wall lime stone $\left(C_{2 w}\right)$, dolomite $\left(C_{1 b}\right)$ and foot wall lime stone $\left(C_{1 d}\right)$ samples were shown in Table 9.4.

\subsubsection{Stress Estimation}

The stress relief method was used to estimate the stress in 1391 level of ore body 10\#. Two measurements position design were showed in Fig. 9.10. In the field, the hollow inclusion measurement is one of stress relief methods; the triaxial stress probes (Institute of geology and geophysics, Chinese academy of science, Beijing, China) can obtained the triaxial stress status at the point in single borehole. The measurement procedures are as follows (Amadei 1983; Amadei and Stephansson 1997).

Table 9.4 Physi-mechanical parameters tested results for rock stratums

\begin{tabular}{l|l|l|l|l|l|l|l}
\hline Samples & $\rho\left(\mathrm{g} / \mathrm{cm}^{3}\right)$ & $\mathrm{E}(\mathrm{GPa})$ & $\mu$ & $R_{c}(\mathrm{MPa})$ & $R_{t}(\mathrm{MPa})$ & $V_{p r}(\mathrm{~m} / \mathrm{s})$ & $V_{p m}(\mathrm{~m} / \mathrm{s})$ \\
\hline Sulfide ores & 4.09 & 15.09 & 0.25 & 86.24 & 3.01 & 4174 & 3757 \\
\hline Oxidized & 4.57 & 14.62 & 0.25 & 85.88 & 4.12 & 4280 & 3890 \\
\hline$C_{2 \mathrm{w}}$ & 2.8 & 17.8 & 0.24 & 74.68 & 6.08 & 5102 & 4151 \\
\hline$C_{1 \mathrm{~b}}$ & 2.78 & 17.17 & 0.26 & 68.66 & 3.07 & 5016 & 4568 \\
\hline$C_{1 \mathrm{~d}}$ & 2.71 & 16.35 & 0.27 & 104.11 & 4.44 & 4955 & 4387 \\
\hline$C_{3 \mathrm{~m}}$ & 2.53 & 8.29 & 0.28 & 20.13 & 1.39 & 3063 & 2580 \\
\hline
\end{tabular}

With kind permission from "PERGAMON-ELSEVIER SCIENCE LTD": Table 1, Wang (2015). All rights reserved 


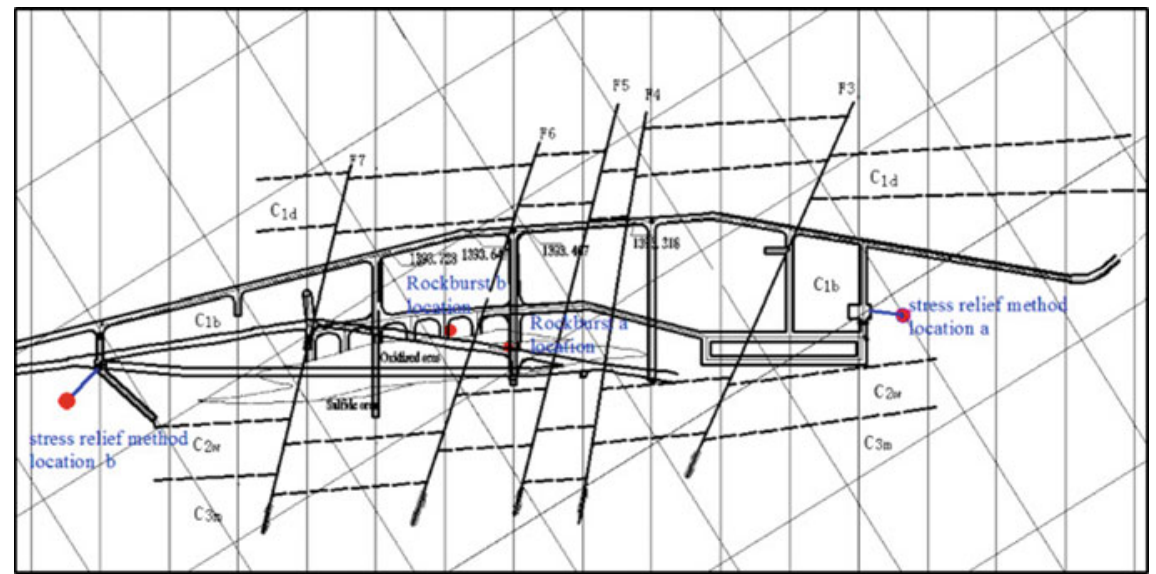

Fig. 9.10 Rockbursts cases and stress relief method positions in 1391 level. With kind permission from “PERGAMON-ELSEVIER SCIENCE LTD": Fig. 2, Wang et al. (2015). All rights reserved

Table 9.5 The measured results of stress in 1391 level of 10\# ore-body

\begin{tabular}{l|l|l|l|l}
\hline $\begin{array}{l}\text { Excavated surrounding } \\
\text { rockmass }\end{array}$ & $\begin{array}{l}\sigma_{1} \\
(\mathrm{MPa})\end{array}$ & $\begin{array}{l}\text { Azimuth } \\
\text { angle }\end{array}$ & $\begin{array}{l}\text { Drilling depth } \\
(\mathrm{m})\end{array}$ & Remarks \\
\hline$C_{1 b}$ & 32.66 & 206.4 & 17.5 & $\begin{array}{l}\text { Stress relief } \\
\text { method }\end{array}$ \\
\hline & 31.73 & 204.4 & 17.5 & \\
\hline
\end{tabular}

With kind permission from "PERGAMON-ELSEVIER SCIENCE LTD": Table 2, Wang et al. (2015). All rights reserved

Firstly, the diameter $130 \mathrm{~mm}$ bore was drilled at the measured point, the depth of borehole was approximate $18 \mathrm{~m}$. Secondly, the diameter $36 \mathrm{~mm}$ pilot hole was drilled in the centre of diameter $130 \mathrm{~mm}$ bore; the depth of hole was approximate $36 \mathrm{~mm}$. Thirdly, the measurement probe was installed in this hole, the initial value was obtained. Fourthly, after curing cement, releasing the stress, the diameter $36 \mathrm{~mm}$ of over coring bore was drilled, the injected water pressed the strain gauges onto the walls of pilot hole. The data were obtained after every drilled $30 \mathrm{~mm}$, the rock core was drawn, over coring was terminated. Fifthly, the procedures $2-4$ were recovered, the acquired data could be verified the previous ones. In this research, the calculated major principal stress was selected the average value, namely, 32.2 MPa.

The results showed that the major principal stress magnitude was the similar as the gravity stress field. However, the azimuth angle of major principal stress was approximately parallel the trend of ore body (N26 E), as shown in Table 9.5. 


\subsubsection{Predicting Rockburst Tendency}

Rockburst tendency is used to confirm the qualitative or quantitative induced possibility of rockburst by the tested method. It is usually divided into four strength grades, namely, no rockburst, weak rockburst, medium rockburst and strong rockburst. The traditional test methods are Barton method, rock brittleness, impact tendency energy and rock integrity. As shown in Table 9.5, it explains the scope of data in the four strength grades using traditional predicting methods. The columns show the data scope of one predicting method bases four strength grades, and the rows show the data scope of strength grades bases the different predicting methods. These terms are related to different parameters with different predicting method, for example, the rock brittleness method is shown as the uniaxial compressive strength and uniaxial tensile strength. However, Barton method is shown as the uniaxial compressive strength and the maximum principal stress. As shown in Table 9.5, the strength grades of rockburst were divided according to the different method.

\subsubsection{Predicting Rockburst Tendency Using Traditional Method}

Several rockburst tendency prediction methods were usually used in practice, the strength grades of methods were shown in Table 8.2. They considered many factors including geostress, rock brittleness, rock integrity and other aspects. These methods were given as follows:

(1) Barton's method (Barton et al. 1974). Here in after referred to as $\alpha$. In general, the elastic strain energy of hard rock was higher than that of soft rock in the high stress zone. Rock strength stress ratio $\alpha=R_{c} / \sigma_{1}$ was generally used to determine the rockburst tendency, where $R_{c}$ was the UCS, and $\sigma_{1}$ was the major principal stress.

(2) Rock brittleness (Tan et al. 1991). Here in after referred to as $B$. The UCS $R_{c}$ and UTS $R_{t}$ were the main factors affecting the rock brittleness. The brittleness coefficient $B=R_{c} / R_{t}$ could be used to predict the possibility of rockburst.

(3) Impact tendency energy (Singh 1988). Here in after referred to as $W_{C F}$, which was determined by the load-deformation curve of rock. Given that $F_{1}$ was the area before the peak and $F_{2}$ was the area after the peak, the impact energy index was calculated as $W_{C F}=F_{1} / F_{2}$. The larger $W_{C F}$ value meant the more likely rockburst would occur.

(4) Rock integrity (Hou and Wang 1989). Here in referred to as $K_{V}$. In the same initial stress conditions, the intact, hard, compact and brittle rock could produce enough energy to cause the local broken rock, to suddenly release and cause rockburst at a certain stress level, as shown in Eq. (9.1). 


$$
K_{V}=\frac{V_{p m}^{2}}{V_{p r}^{2}}
$$

where $K_{V}$ is the rock integrity coefficient; $V_{p m}$ is the rockmass elastic wave speed, $\mathrm{m} / \mathrm{s} ; V_{p r}$ is the rock elastic wave speed, $\mathrm{m} / \mathrm{s}$.

This grade classification was generally obtained based on the range of predicted value. Each predicting methods of rockburst tendency were divided into four strength grades, and the specific grade situation was shown in Table 9.3.

\subsubsection{Predicting Rockburst Tendency Using the Proposed Model}

Fuzzy matter-element theory was applied to evaluate multi-index incompatible problem. It is used to solve to the problem with the method of visualization. Fuzzy matter-element model of rockburst tendency was established (Wang et al. 2015), and the steps of predicting rockburst tendency were shown as follows.

(1) Establishing fuzzy matter-element

1. According to the strength grades of rockburst tendency (as shown in Table 9.3), the obtained physi-mechanical parameters and stress (as shown in Tables 9.2 and 9.3) were used to establish the fuzzy matter-element matrix for rockburst evaluation.

$$
R_{(4,10)}=\left[\begin{array}{lllllll} 
& \text { Sulphide ores } & \text { Oxidized ores } & C_{2 w} & C_{1 b} & C_{1 d} & C_{3 m} \\
B & 28.680 & 20.825 & 12.291 & 22.354 & 23.433 & 14.490 \\
R_{c} / \sigma_{1} & 2.678 & 2.667 & 2.319 & 2.132 & 3.233 & 0.625 \\
W_{C F} & 1.210 & 2.210 & 2.160 & 1.120 & 0.910 & 1.210 \\
K_{V} & 0.810 & 0.870 & 0.664 & 0.833 & 0.784 & 0.711
\end{array}\right.
$$

No rockburst Weak rockburst Medium rockburst Strong rockburst $]$

$\left.\begin{array}{llll}>40.000 & 26.700-40.000 & 14.500-26.700 & <14.500 \\ >10.000 & 5.000-10.000 & 2.500-5.000 & <2.500 \\ <1.000 & 1.000-2.000 & 2.000-3.000 & >3.000 \\ <0.500 & 0.500-0.600 & 0.500-0.750 & >0.750\end{array}\right]$

2. According to the preferable membership grade principle, the model was given in the larger the more optimal principles in the intensity of rockburst in creases with the decreased of $R_{c} / \sigma_{1}$ and $B$. On the contrary, as the in tensity of rockburst increased with the rise of $W_{C F}$ and $K_{V}$, the model adopted the smaller them ore optimal principle. Namely, $\mu_{i j}=X_{i j} \min / X_{i j}$. The composite fuzzy matter-element matrix was established as follow: 
Table 9.6 Predicting rockburst tendency grades using traditional method

\begin{tabular}{l|l|l|l|l}
\hline Strength grade & $B$ & $\alpha$ & $W_{C F}$ & $K_{V}$ \\
\hline No rockburst & $>40.0$ & $>10.0$ & $<1$ & $<0.50$ \\
\hline Weak rockburst & $26.7-40.0$ & $5.0-10.0$ & $1-2$ & $0.50-0.60$ \\
\hline Medium rockburst & $14.5-26.7$ & $2.5-5.0$ & $2-3$ & $0.60-0.75$ \\
\hline Strong rockburst & $<14.5$ & $<2.5$ & $>3$ & $0.75-1.00$ \\
\hline
\end{tabular}

With kind permission from "PERGAMON-ELSEVIER SCIENCE LTD": Table 3, Wang et al. (2015). All rights reserved

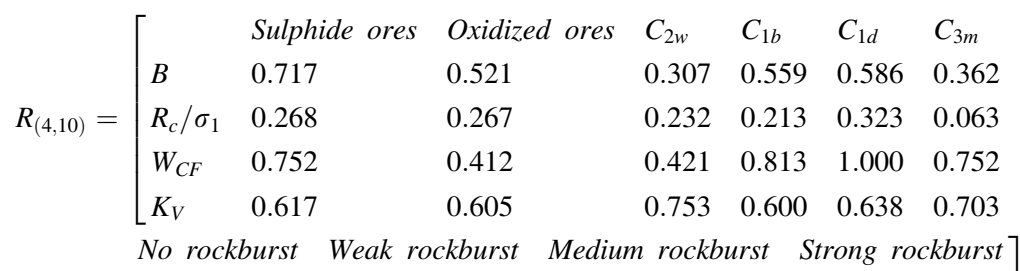

$\begin{array}{llll}>1.000 & 0.668-1.000 & 0.363-0.668 & <0.363 \\ >1.000 & 0.500-1.000 & 0.250-0.500 & <0.250 \\ <0.910 & 0.455-0.910 & 0.303-0.455 & >0.303 \\ <1.000 & 0.833-1.000 & 0.667-0.833 & >0.667\end{array}$

3. According to the composite fuzzy matter-element matrix, the preferable membership grade of index extract value 1 for predicting closer to reality, the different square were composite fuzzy elements were established (Table 9.6):

$$
\begin{aligned}
& R_{(4,10)}= {\left[\begin{array}{lllllll} 
& \text { Sulphide ores } & \text { Oxidized ores } & C_{2 w} & C_{1 b} & C_{1 d} & C_{3 m} \\
B & 0.080 & 0.230 & 0.480 & 0.195 & 0.172 & 0.407 \\
R_{c} / \sigma_{1} & 0.536 & 0.538 & 0.590 & 0.619 & 0.458 & 0.879 \\
W_{C F} & 0.062 & 0.346 & 0.619 & 0.035 & 0.000 & 0.062 \\
K_{V} & 0.147 & 0.156 & 0.061 & 0.160 & 0.131 & 0.088 \\
\text { No rockburst } & \text { Weak rockburst } & \text { Medium rockburst } & \text { Strong rockburst }
\end{array}\right] } \\
&
\end{aligned}
$$

$\left.\begin{array}{llll}0.000 & 0.000-0.111 & 0.111-0.406 & >0.406 \\ 0.000 & 0.000-0.250 & 0.250-0.563 & >0.563 \\ 0.000 & 0.008-0.297 & 0.297-0.485 & >0.485 \\ 0.000 & 0.000-0.028 & 0.028-0.111 & >0.111\end{array}\right]$


(2) Calculating the weight coefficient using the entropy method

The evaluation matrix was normalized and the entropy $H_{i}$ and $w_{i}$ were calculated according to Eqs. (8.12)-(8.16).

$$
\begin{gathered}
B_{i j}=\left[\begin{array}{ccccccc} 
& \text { Sulphide ores } & \text { Oxidized ores } & C_{2 w} & C_{1 b} & C_{1 d} & C_{3 m} \\
B & 0.000 & 0.374 & 1.000 & 0.286 & 0.229 & 0.817 \\
R_{c} / \sigma_{1} & 0.186 & 0.190 & 0.314 & 0.383 & 0.000 & 1.000 \\
W_{C F} & 0.178 & 1.000 & 0.968 & 0.102 & 0.000 & 0.178 \\
K_{V} & 0.865 & 0.965 & 0.000 & 1.000 & 0.711 & 0.274
\end{array}\right] \\
H_{i}=\left[\begin{array}{lllll}
0.984 & 0.986 & 0.977 & 0.984
\end{array}\right]^{T}(\mathrm{i}=1,2,3,4) \\
w_{i}=\left[\begin{array}{lllll}
0.228 & 0.209 & 0.338 & 0.226
\end{array}\right]^{T} \quad(\mathrm{i}=1,2,3,4)
\end{gathered}
$$

(3) The closeness degree calculation

The composite fuzzy matter-element for closeness degree was established by Eq. (8.16):

$$
\begin{aligned}
R_{\rho H}= & {\left[\begin{array}{llllll}
\text { Sulphide ores } & \text { Oxidized ores } & C_{2 w} & C_{1 b} & C_{1 d} & C_{3 m} \\
0.571 & 0.437 & 0.401 & 0.529 & 0.595 & 0.437 \\
& \text { No rockburst } & \text { Weak rockburst } & \text { Medium rockburst } & \text { Strong rockburst } \\
& >0.904 & 0.571-0.948 & 0.368-0.571 & <0.368
\end{array}\right] }
\end{aligned}
$$

\subsubsection{Predicting Model of Fuzzy Matter-Element Theory}

\subsubsection{Comparison and Analysis of Predicting Model}

The results of rockburst tendency for surrounding rockmass and ore body were calculated by these four predicting methods in the 10\# ore body. The calculation results were shown in Table 9.7. Based on the calculations of closeness degree, the evaluation of rockburst tendency for the surrounding rockmass in 10\# ore body was obtained using fuzzy matter-element evaluation model, as shown in Table 9.8. There results showed that $C_{1 d}$ had weak rockburst tendency. The sulfide ores, oxidized ores, $C_{2 w}, C_{1 b}$ and $C_{3 m}$ had medium rockburst tendency. There results were compared with those calculations obtained.

As shown in Table 9.8. It was concluded that the rockmass in the 1331-1451 levels of 10 \# ore-body had the possibility of medium rockburst in sulfide ores, oxide minerals, $C_{2 w}, C_{1 b}$ and $C_{3 m}$, and the weak rockburst maybe be occurred in the $C_{1 d}$. 
Table 9.7 Results of each rockburst predicting methods in 10\# ore body

\begin{tabular}{l|l|l|l|l}
\hline Ore and rockmass & $B$ & $\alpha$ & $W_{C F}$ & $K_{V}$ \\
\hline Sulfide ores & 28.680 & 2.678 & 1.210 & 0.810 \\
\hline Oxidized ores & 20.825 & 2.667 & 2.210 & 0.827 \\
\hline$C_{2 w}$ & 12.291 & 2.319 & 2.160 & 0.664 \\
\hline$C_{1 b}$ & 22.354 & 2.132 & 1.120 & 0.833 \\
\hline$C_{1 d}$ & 23.433 & 3.233 & 0.910 & 0.784 \\
\hline$C_{3 m}$ & 14.490 & 0.625 & 1.210 & 0.711 \\
\hline
\end{tabular}

With kind permission from "PERGAMON-ELSEVIER SCIENCE LTD": Table 4, Wang et al. (2015). All rights reserved

Table 9.8 Comparison of predicting results

\begin{tabular}{l|l|l|l|l|l}
\hline $\begin{array}{l}\text { Ore and } \\
\text { rockmass }\end{array}$ & $B$ & $\alpha$ & $W_{C F}$ & $K_{V}$ & $\begin{array}{l}\text { Fuzzy Matter element } \\
\text { model }\end{array}$ \\
\hline Sulfide ores & Weak & Medium & Weak & Strong & Medium \\
\hline Oxidized ores & Medium & Medium & Medium & Strong & Medium \\
\hline$C_{2 w}$ & Strong & Strong & Medium & Medium & Medium \\
\hline$C_{1 b}$ & Medium & Strong & Weak & Strong & Medium \\
\hline$C_{1 d}$ & Medium & Medium & No & Strong & Weak \\
\hline$C_{3 m}$ & Strong & Strong & Weak & Medium & Medium \\
\hline
\end{tabular}

With kind permission from "PERGAMON-ELSEVIER SCIENCE LTD": Table 4, Wang et al. (2015). All rights reserved

\subsubsection{Data Analysis and Discussions}

In 10\# ore body, rockburst occurred at the drift, 5 panel, $1391 \mathrm{~m}$ level on Oct $11 \mathrm{st}$, 2012 (Fig. 9.11a). It was approximately $100 \mathrm{t}$ in the $C_{1 b}$ rock strata. The other rockburst occurred at the drift, 7 panel, $1391 \mathrm{~m}$ level on Nov 28th, 2013 (Fig. 9.11b). It was approximately $50 \mathrm{t}$ in the $C_{1 b}$ rock strata.

The mining engineering only excavated in the $C_{1 b}$ stratum, sulfide ore sand oxidized ores. As shown in Fig. 9.11. The other stratums indiced of predicting model were obtained by the borehole in the laboratory and field. So the case rockbursts could only be occurred in the $C_{1 b}$, sulfide ore sand oxidized ores. However, the sulfide ore sand oxidized ores were mined by the immediate paste backfilling, the ores stratums stress could be controlled by the filling body, and it might be not occur the rockbursts.

The grade for two rockburst tendency of $C_{1 b}$ was different according to different evaluation methods. It was strong with Barton method ( $\alpha$ method) and rock integrity method ( $K_{V}$ method), medium with fuzzy matter-element model and rock brittleness method ( $B$ method), weak with impact tendency energy method ( $W_{C F}$ method). To acquire the compared different results, the research concluded that these results were related with the considered factors of different method. As 
(a)

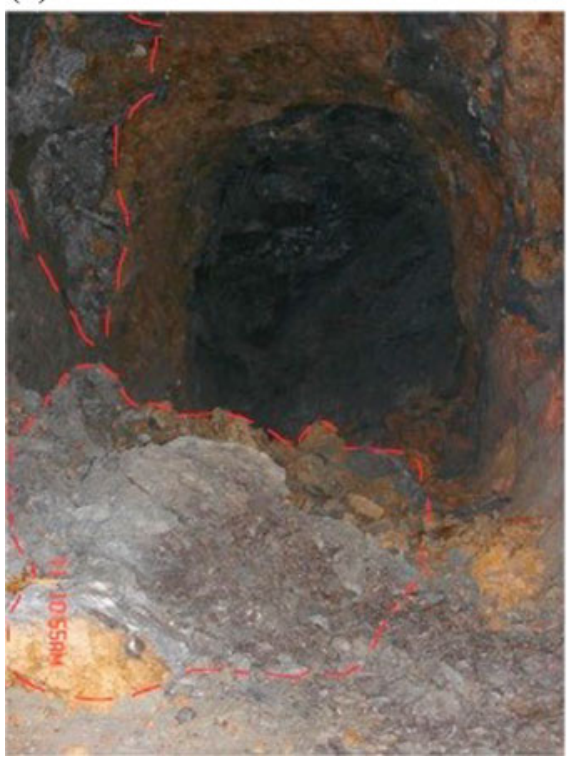

(b)

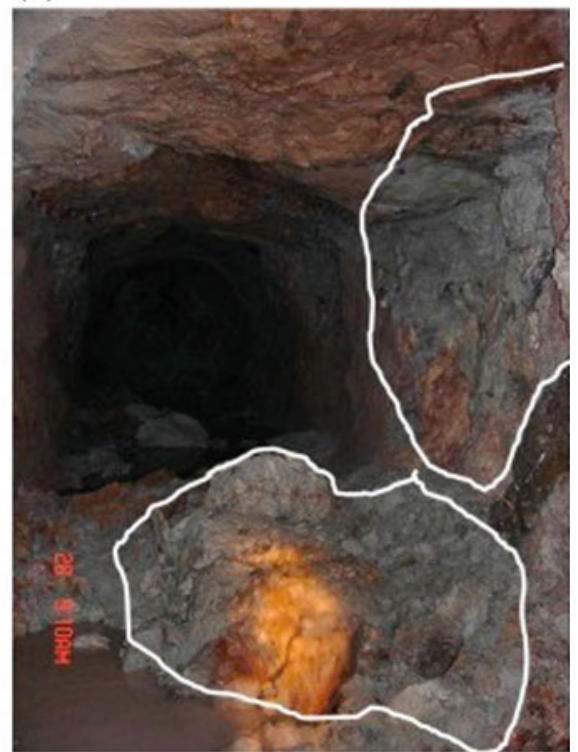

Fig. 9.11 Rockbursts on site. With kind permission from "PERGAMON-ELSEVIER SCIENCE LTD": Fig. 3, Wang et al. (2015). All rights reserved

follow, Barton method only considered the UCS and the major principal stress, but the brittleness and integrity of rock were not included in the model. However, 10\# ore body and other rock stratums were not particularly intact, the predicting result of Barton method might be slightly higher than the actual value, its predicting result was strong rockburst grade; Rock brittleness method didn't contain the major principal stress, which was the base factor for the rockburst tendency. It was important that the stress caused the accumulated energy of rockmass, its predicting result was medium rockburst grade, and this result was the same as the fuzzy matter-element model. However, in other predicting result of rock brittleness method, they might be away from the true value because of lacking the most important factor in stress; Impact tendency energy method only considered the before and after peak value, rock brittleness was only one of factors of rockburst tendency. However, the parameters, such as the stress and the intact degree, were important factors in the predicting result. In this research, we could find that the curve was mitigated. So the predicting of impact tendency energy method was quiet low; Rock integrity method didn't include the major principal stress, rock brittleness and the impact tendency energy, which were the base factors of energy accumulation. That is to say, these traditional methods exited the limitations for rockburst tendency. However, fuzzy matter-element model were obtained using all factors of traditional method, the predicting result using fuzzy matter-element method for rockburst was medium in 1391 level of $C_{1 b}$ rock strata in 10\# ore body, 
as shown in Table 9.8. For exposing rockmass and ore body in this mine depth, the case results showed that fuzzy matter-element predicting was right. Because no other rock stratums exposed, such as $C_{2 w}, C_{1 b}$ and $C_{3 m}$, we only obtained the predicting results from $C_{1 b}$, so the proposed model needs further validation. However, the two cases showed that the proposed model could predict rockburst in a reliable mode; this is a meaningful discovery for predicting rockburst tendency.

\subsection{Conclusion}

A large number of AE information before and during rockburst was obtained using AE experiments of rock under cyclic load/unload. Through CT scanning technology, we reconstructed the fissured rock in three-dimensions and built the cracks growth factor model. Furthermore, the predicting key points of precursor information were obtained by analyzing the nonlinear dynamics evolution pattern of rock cracks and fissured rock mass.

Many models and cases of precursor information prediction of rockburst have been described in this chapter, such as Bayesian model, Fuzzy matter-element model, TDF identification, TM identification, information entropy and $b$ value, load/unload response ratio, infrared radiation, CAV and MS rate, spatial-temporal-energy evolution model of rock failure, etc. These cases verified that the proposed model and theory could predict rockburst in a reliable mode. This is a meaningful discovery for predicting rockburst.

It was worth mentioning that we used a Bayesian multi-parameter model to predict rockburst tendency by improving upon other traditional prediction methods. Cases results show that the proposed predicting models were more reliable than those traditional methods. The results demonstrated that the multivariable Bayesian model was highly accurate in predicting rockburst tendency. We recommend that Bayesian models to predict rockburst in field.

In addition, compared with traditional methods, fuzzy matter-element method was verified based on the rockburst in field. The results showed that the fuzzy matter-element was very prominent for the predicting rockburst intensity. Obtaining more accurate results of fuzzy matter-element model, we proposed in put many parameters. This may improve predicting quality.

Finally, we recommend the proposed predicting methods of rockburst for field cases. 


\section{References}

Amadei B (1983) Number of bore holes to measure the state of stress in situ by overcoring. In: The 24th US Symposium on Rock Mechanics (USRMS). College Station, Assoc Eng Geol Publ, pp 87-98

Amadei B, Stephansson O (1997) Rock stress and its measurement. Chapman\&Hall, London

Barton NR, Lien R, Lunde J (1974) Engineering classification of rockmasses for the design of tunnel support. Rock Mech 6:189-239

Cheng Y, Jiang F, Cheng J (2006) Preliminary study on microseismic monitoring of mine tremor induced by key strata movement. J Coal Ind 31(3):273-275

He SL, Wang CL (2010) Analysis of influencing factors of rockburst in deep mining in Huize lead-zinc mine. Miner Eng Res 25:21-23 (in Chinese)

Hou FL, Wang MQ (1989) Criterion and prevention measures on rockburst in circular tunnel. In: Proceedings of the 2nd national rock mechanics and engineering (Chinese Rock Mech Eng). Knowledge Press, Beijing

Li S, Yin X, Deng W (2005) The research of Fankou mine multi-channel microseismic monitoring system and its application. J Rock Mech Eng 24(12):2058-2059

Liu J (2008) A new rock mass stability monitoring system based on AE and microseismic technology and its application. Nonferrous Metal Mine Sect 60(4):32-35

Singh SP (1988) Burst energy release index. Rock Mech and Rock Eng 21(2):149-155

Tan Y, Sun GZ, Guo ZH (1991) A composite index K(rb) criterion for the ejection characteristics of the burst rock. Chin J Geol 2:193-200

Wang CL (2013) Identification and early warning method for rock mass instability. Disaster Adv 6 (12):101-105

Wang CL (2014) Identification of early-warning key point for rockmass instability using acoustic emission/microseismic activity monitoring. Int J Rock Mech Min Sci 71:171-175

Wang CL, Wu AX, Liu XH (2009) Mechanisms of microseismic events occurred in deep heard-rock mine of China. In: Proceedings seventh international symposium on rockburst and seismicity in mines, Liaoning Dalian

Wang CL, Wu AX, Lu H, Bao TC, Liu XH (2015) Predicting rockburst tendency based on fuzzy matter-element model. Int J Rock Mech Min Sci 75:224-232

Open Access This chapter is licensed under the terms of the Creative Commons Attribution-NonCommercial 4.0 International License (http://creativecommons.org/licenses/by-nc/ 4.0/), which permits any noncommercial use, sharing, adaptation, distribution and reproduction in any medium or format, as long as you give appropriate credit to the original author(s) and the source, provide a link to the Creative Commons license and indicate if changes were made.

The images or other third party material in this book are included in the book's Creative Commons license, unless indicated otherwise in a credit line to the material. If material is not included in the book's Creative Commons license and your intended use is not permitted by statutory regulation or exceeds the permitted use, you will need to obtain permission directly from the copyright holder. 